Soil fauna in lowland rainforest and agricultural systems of Sumatra: Changes in community composition and trophic structure with focus on Collembola

\author{
Dissertation \\ zur Erlangung des mathematisch-naturwissenschaftlichen Doktorgrades \\ "Doctor rerum naturalium" \\ der Georg-August-Universität Göttingen \\ im Promotionsprogramm Biologie \\ der Georg-August University School of Science (GAUSS) \\ vorgelegt von \\ Winda Ika Susanti
}

aus Indonesien

Göttingen, 2021 


\section{$\underline{\text { Betreuungsausschuss }}$}

Stefan Scheu

J.F. Blumenbach Institute of Zoology and Anthropology, Georg-August-University Göttingen

Christoph Bleidorn

Dep. Animal Evolution and Biodiversity, Georg-August-University Göttingen

\section{Mitglieder der Prüfungskommission}

Referent: Stefan Scheu

Korreferent: Christoph Bleidorn

\section{Weitere Mitglieder der Prüfungskommission:}

Mark Maraun

J.F. Blumenbach Institute of Zoology and Anthropology, Georg-August-University Göttingen

Klaus Hövemeyer

J.F. Blumenbach Institute of Zoology and Anthropology, Georg-August-University Göttingen

Sven Bradler

Dep. Animal Evolution and Biodiversity, Georg-August-University Göttingen

Holger Kreft

Dept. Biodiversity, Macroecology \& Biogeography, Georg-August-University Göttingen

Tag der mündlichen Prüfung: 19 October 2021 


\section{TABLE OF CONTENT}

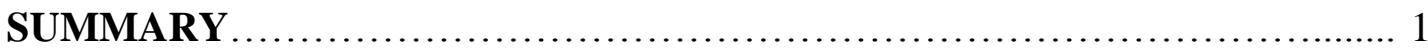

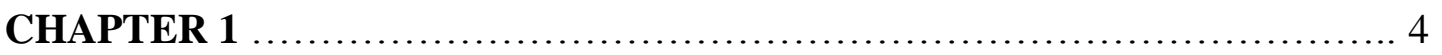

\section{GENERAL INTRODUCTION}

Conversion of rainforest into rubber and oil palm plantations in Indonesia ......... 4

Effect of rainforest conversion on soil animals ................................ 4

Collembola as model organisms ......................................... 5

Effect of environmental factors and seasonal variation on Collembola ............. 6

Functional traits of Collembola ........................................... 7

Fatty acid analysis as tool for soil food-web analysis ........................ 8

Investigating trophic position of and utilization of food resources by Collembola using stable isotope analysis ............................................. 9

Methodology, hypotheses and objectives ................................. 10

\section{CHAPTER 2}

CONVERSION OF RAINFOREST TO OIL PALM AND RUBBER PLANTATIONS ALTERS ENERGY CHANNELS IN SOIL FOOD WEBS

Abstract ............................................................ 16

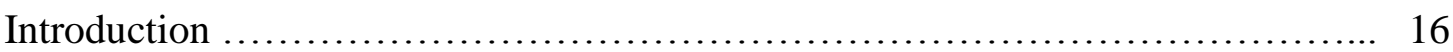

Material and methods ................................................. 19

Results

Food resources of different groups of soil fauna .............................. 24

Effect of land-use change on food resources of soil fauna ..................... 25

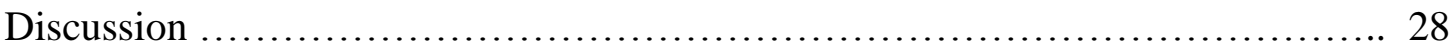

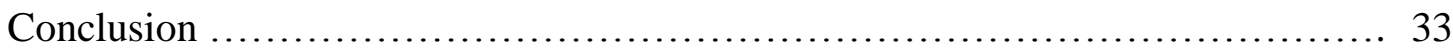

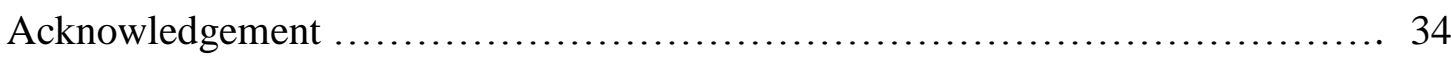

\section{CHAPTER 3}

CONVERSION OF RAINFOREST INTO OIL PALM AND RUBBER PLANTATIONS AFFECTS THE FUNCTIONAL COMPOSITION OF LITTER AND SOIL COLLEMBOLA

Abstract ............................................................... 50

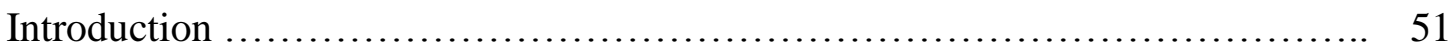


Material and methods 53

Results

Density, species richness, functional diversity and functional dispersion 61

Species and trait composition of communities 64

Correlation between environmental factors and community composition 67

Discussion 71

Conclusion 76

Acknowledgement 77

\section{CHAPTER 4}

\section{TROPHIC NICHE DIFFERENTIATION AND UTILISATION OF FOOD RESOURCES IN COLLEMBOLA IS ALTERED BY RAINFOREST CONVERSION TO PLANTATION SYSTEMS}

Abstract 104

Introduction 105

Material and methods 107

Results

Community-level changes with land use 111

Trophic niche differentiation among species

Differences among Collembola life forms and families 116

Discussion 118

Conclusion 122

Acknowledgement

\section{CHAPTER 5}

SEASONAL FLUCTUATIONS OF LITTER AND SOIL COLLEMBOLA AND THEIR DRIVERS IN RAINFOREST AND PLANTATIONS

Abstract 130

Introduction 131

Material and methods

Results

Variations in density of total Collembola with land-use system, season, layer and life form

Variations in density of Collembola life forms with land-use system, 
layer and season .............................................................. 141

Seasonal variations in community composition of Collembola

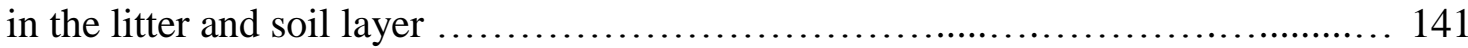

Environmental factors related to Collembola community composition ............... 143

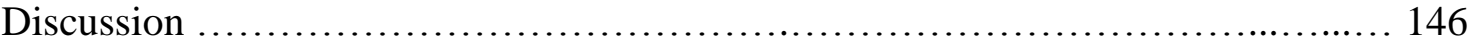

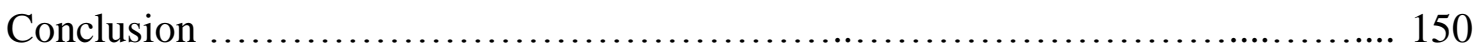

Acknowledgement....................................................... 150

\section{CHAPTER 6}

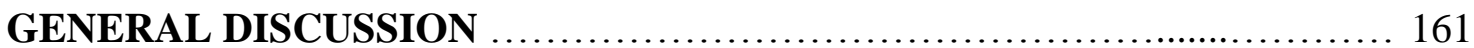

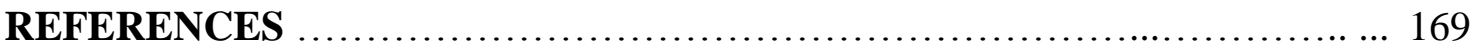

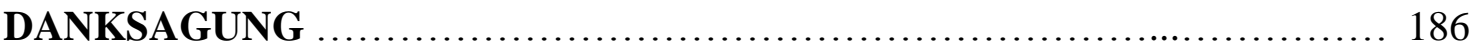

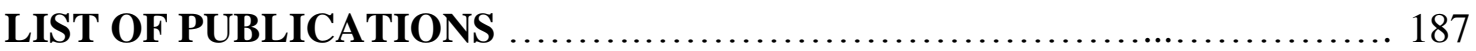

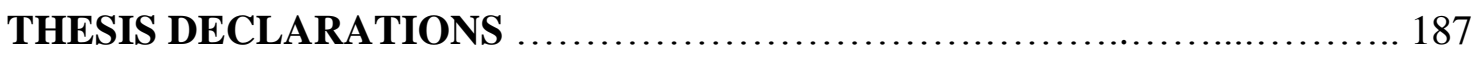

CURRICULUM VITAE ................................................ 188 


\section{SUMMARY}

Rainforest conversion to agricultural plantations such as rubber and oil palm in Indonesia is increasing in the last decades. In this thesis, I investigated effects of rainforest conversion to rubber and oil palm plantations in Jambi, Sumatra, Indonesia. The study area serves as model region for investigating consequences of land-use changes on biodiversity, the channeling of energy through soil food webs and changes in trophic niches of one dominant group of soil mesofauna, Collembola. Collembola are among the most abundant arthropods inhabiting the belowground system sensitively responding to changes in vegetation and soil conditions.

In Chapter 2, consequences of the conversion of rainforest into plantations on the channelling of energy through soil food webs was investigated by using neutral lipid fatty acid analysis from six dominant soil fauna taxa. Rainforest conversion changed the biomarker FA composition of soil fauna at the community level. Conversion of rainforest into oil palm plantations enhanced the plant energy channel and reduced the bacterial energy channel. The changes in energy distribution within soil food webs may have significant implications for the functioning of tropical ecosystems and their response to environmental changes.

In Chapter 3, I investigated effects of rainforest conversion on the density, species richness, functional and community composition of soil and litter Collembola. Land-use change negatively affected Collembola communities in the litter layer, but the impact was less pronounced in the soil layer. Water content and $\mathrm{pH}$ were identified as important factors driving Collembola community composition. Several pantropical genera of Collembola (i.e., Isotomiella, Pseudosinella and Folsomides) dominated across land-use systems, reflecting their high environmental adaptability or efficient dispersal calling for studies on their ecology and genetic diversity. The decline in species richness and density of litter-dwelling Collembola with the conversion of rainforest into plantation systems calls for management practices mitigating negative effects of the deterioration of the litter layer in rubber plantations, but even more in oil palm plantations. Specific traits, such as larger size, patterned or intensive pigmentation and number of ocelli may explain the adaptation of Collembola species to the environmental changes in particular to changes in soil water content and $\mathrm{pH}$.

In Chapter 4, the effect of rainforest conversion on the trophic niche structure of Collembola was investigated using stable isotope analysis. Across Collembola species, $\Delta{ }^{13} \mathrm{C}$ 
values (calibrated to litter) were highest in rainforest suggesting more pronounced processing of litter resources by microorganisms and consumption of these microorganisms by Collembola in this system. Lower $\Delta^{13} \mathrm{C}$ values, but high $\Delta^{13} \mathrm{C}$ variation in Collembola in oil palm plantations indicated that Collembola shifted towards herbivory and used more variable resources in this system. Small range in $\Delta^{15} \mathrm{~N}$ values in Collembola species in monoculture plantations in comparison to rainforest indicated that conversion of rainforest into plantations is associated with simplification in the trophic structure of Collembola communities. Across the studied ecosystems, atmobiotic species occupied the lowest, whereas euedaphic Collembola species occupied the highest trophic position, resembling patterns in temperate forests. A dominant species of the euedaphic life form and of high trophic position (Pseudosinella sp.1) was found in high abundance across rainforest and plantations suggesting that this species is best adapted to the studied land-use changes. Euedaphic species generally appear to be better adapted to cope with seasonal fluctuations than other life forms.

Additionally, in Chapter 5, I investigated seasonal fluctuations of Collembola density and community composition in rainforest and plantation systems. Collembola density in the litter layer, was at a maximum at the beginning of the wet season. Euedaphic and hemiedaphic species, living in the lower litter layers and soil, fluctuated less with season than epedaphic and atmobiotic species, living in upper litter layers. Differences in community composition among land-use systems were most pronounced at the beginning of the dry season (June). Collembola community composition changed with season in all landuse systems, with the differences being more pronounced in the litter than in the soil layer. In line with the results from Chapter 3 , water content and $\mathrm{pH}$ were identified as important factors driving Collembola community composition across seasons.

Overall, rainforest conversion to rubber and oil palm have negative impacts on soil and litter Collembola biodiversity, alter the trophic structure of Collembola communities and the channeling of energy through soil food webs. To reduce the negative impacts of rainforest conversion into plantation systems as revealed in this thesis, improved management of agricultural plantations is needed.

Key words: soil fauna, Collembola, fatty acids, stable isotope, rainforest conversion 


\section{CHAPTER 1}

\section{GENERAL INTRODUCTION}
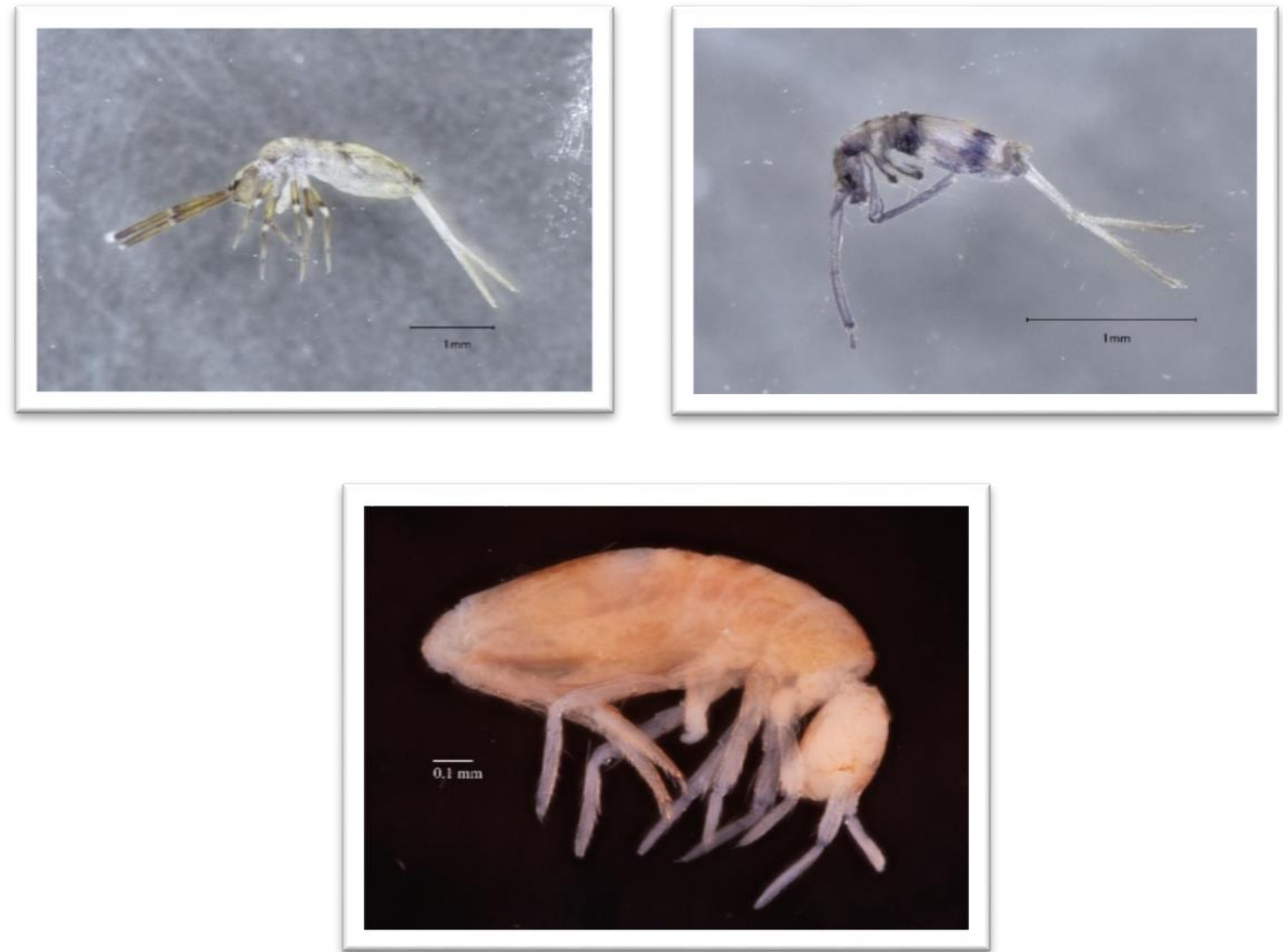

Photos by Winda Ika Susanti 


\section{GENERAL INTRODUCTION}

\section{Conversion of rainforest into rubber and oil palm plantations in Indonesia}

In tropical Asia, a rapidly growing population and agricultural expansion collide with one of the highest levels of biodiversity and endemism worldwide (Myers et al., 2000; Sodhi et al., 2004). Rainforests in Southeast Asia have been logged on a large scale since the mid- $20^{\text {th }}$ century, usually followed by subsequent transformation of logged-over rainforests into cash crop monocultures (Wilcove and Koh, 2010) such as acacia, rubber and oil palm. In Indonesia, this process has accelerated during the past decades, where annual loss in rainforest cover was estimated at 0.84 million hectares for the year 2012, the highest worldwide (Margono et al., 2014). Large-scale conversion of rainforest for agricultural use is particularly evident on the island of Sumatra, which experiences the highest primary rainforest cover loss in all of Indonesia (Laumonier et al., 2010; Miettinen et al., 2011). The conversion of tropical rainforest into plantations is associated with the degradation and destruction of habitats, which is among the most significant and immediate threats to biodiversity worldwide (Titeux et al., 2016). Experiencing massive deforestation in the last decades, Sumatra represents a model region to investigate the effect of rainforest conversion into plantation systems on biodiversity and ecosystem functioning at local and regional scales (Clough et al., 2016; Drescher et al., 2016).

\section{Effect of rainforest conversion on soil animals}

Soil harbors a large fraction of terrestrial biodiversity with the majority of arthropods living there at last for part of their life cycle. Among described organisms, soil animals comprise around $23 \%$ of all species (Decaëns et al., 2006). However, their contribution to global biodiversity is likely underestimated since soil organisms are poorly studied in comparison to aboveground animal groups (André et al., 2002; Barrios, 2007). Different groups of soil organisms are associated with major ecosystem services provided by soil including water storage, decomposition and nutrient cycling, detoxification of toxicants, and control of pathogenic organisms (Doran et al., 2000). Soil invertebrates regulate the majority of soil processes. Different species of meso- and macrofauna alter microbial community composition and modify the structure of soil by burrowing and grazing activities (Lavelle et al., 2006). Soil organisms are intimately interrelated with the other ecosystem components, e.g. by affecting nutrient supply for 
plants (Wardle et al., 2004). This makes soil animals and interactions in soil communities important components of ecosystem models (De Vries et al., 2013). Linking soil communities to ecosystem functions is the major step to achieve better understanding of biological systems. In tropical regions, however, research on soil animal communities is strongly lacking behind with scarce knowledge on species composition and links within communities of natural and transformed ecosystems.

The conversion of tropical rainforest into plantations is associated with the degradation and destruction of habitats (Titeux et al., 2016), resulting in a loss of plant and animal biodiversity aboveground (Fitzherbert et al., 2008; Clough et al., 2016) and belowground as shown for nematodes (Krashevska et al., 2019), testate amoebae (Krashevska et al., 2016), spiders (Potapov et al., 2020b), Collembola (Deharveng 1996; Filser et al., 2002; Widrializa et al., 2015) and litter macroinvertebrates (Barnes et al., 2014), and also associated with changes in ecosystem functioning (Sousa et al., 2006; Fitzherbert et al., 2008; Gilbert, 2012; Barnes et al., 2014; Clough et al., 2016).

Studies estimating soil biodiversity and the consequences of its loss for species interactions in soil are scarce in Indonesia, which contrasts the pronounced deforestation and intensification of land-use over the last decades. Scientific progress is partly constrained by the generally poor knowledge on the tropical soil fauna. However, development of new methods, such as stable isotope and fatty acid analysis can overcome these constraints by providing information on trophic interactions between organisms (Tiunov, 2007; Brose and Scheu, 2014). My research aimed at revealing changes in abundance, community structure and trophic interactions of soil fauna connected with intensive agricultural land use such as rubber and oil palm plantations in Sumatra, Indonesia. In this study, I will specifically focus on the species and trait composition of Collembola communities from litter and soil layers.

\section{Collembola as model organisms}

Collembola (springtails) are early-derived Hexapoda characterized, among other features, by a "jumping organ" (furca) at the ventral side of the abdomen. The evolution of the furca likely contributed to species diversification (Beutel et al., 2017). The furca presumably evolved to escape predators, but it is reduced or lost in some soil-dwelling species. Another feature that characterizes Collembola is the ventral tube, which has functions in fluid balance and is presumably important because the habitats Collembola 


\section{Chapter 1}

live in are usually moist, such as soil, tree canopies, aquatic surfaces, glaciers and caves (Hopkin 1997). In soil, Collembola are among the most abundant and diverse mesofauna with densities of typically 10,000-100,000 individuals per square meter (Petersen and Luxton 1982). They are decomposers, feeding mainly on fungi and decaying organic matter, but may also function as omnivores consuming a variety of food resources (Scheu and Falca 2000; Berg et al., 2004; Chahartaghi et al., 2005). They regulate microbial activity and therefore contribute to decomposition processes and nutrient cycling (Rusek 1998; Schaefer et al., 2009).

As decomposers, Collembola occupy a crucial place in soil communities by feeding on various food sources and being prey for many predator (Rusek, 1998). Collembola widely affect soil processes such as decomposition rates and nitrogen mineralization, and shape the community composition of microorganisms, primarily fungi (Persson, 1989; Ek et al., 1994; Kanters et al., 2015). Due to large morphological diversity, different groups of Collembola occupy different trophic niches and perform different ecosystem functions (Potapov et al., 2016). However, little is known about the role of different Collembola species and factors that structure Collembola communities in tropical ecosystems. Application of fertilizers, pesticides and mechanical disturbance of soil in landscapes with intensive agriculture lead to loss of soil biodiversity and connected ecosystem functions (Matson et al., 1997). In tropical ecosystems, significant loss in abundance, diversity and biomass of important macrofauna groups, such as termites and earthworms, has been shown in agricultural systems relative to natural forest (Lavelle et al., 1987). Loss of species inevitably alters the interactions in the soil food web and change the flow of energy through soil communities (Barnes et al., 2014). In turn, the loss of interactions reduces stability of the ecosystem and may facilitate pest invasions (Tylianakis et al., 2007).

\section{Effect of environmental factors and seasonal variation on Collembola}

Community composition of litter and soil Collembola can be linked to both biotic and abiotic factors. Salamon et al. (2008) also found dominant functional groups/species of Collembola to mainly depend on abiotic factors such as $\mathrm{pH}$ and soil water content. Pollierer et al. (2017) reported that $\mathrm{pH}$ correlated negatively with fungal and arbuscular mycorrhizal density driving Collembola community structure. However, soil Collembola metacommunities have a less restricted distribution along environmental gradients (Liu et al., 2019). Variations in Collembola abundance additionally depend on microbial 
biomass (Filser et al., 2002). Soil pH also is an important factor driving Collembola community composition in contaminated soils (Errington et al., 2018). Furthermore, another study also reported that temperature and moisture can exert strong effects on Collembola community composition (Petersen, 2011). The study by Makkonen et al. (2011) which manipulated the soil moisture regime reported that certain Collembola species are better adapted to drought conditions. The environmental factors both biotic and abiotic may also change followed changes of land-use system, exacerbating the effects of habitat destruction.

Density and community composition of Collembola in soil and litter can be influenced by seasonal variations throughout the year. The study by Hutson and Veitch (1987) reported that the density of Collembola and Acari in litter and soil layer in South Australian forests followed a seasonal pattern. The study by Xu et al. (2012) also reported that seasonal drought and air temperature affected the genus level richness, abundance and biomass of Collembola communities in an oak forest. Similarly, seasonal fluctuations in community structure of Collembola was reported by Chagnon et al. (2000) in sugar maple forest in Canada, which revealed that epigeic species are more affected by seasonal changes than dominant endogeic species. Further, in the tropics effects of seasonal fluctuations on Collembola density and community were reported showing that Collembola density declines during the dry season (Palacios-Vargas and CastañoMeneses, 2003; Wiwatwitaya and Takeda, 2005; Muturi et al., 2009). In line with this, Mayvan et al. (2015) reported that the density and diversity of Collembola in temperate forests in Iran were lowest in the dry season.

\section{Functional traits of Collembola}

Functional traits are properties of individuals which influence their performance and fitness (Violle et al., 2007; Pey et al., 2014). Ecologically, functional traits regulate the occurrence of species in habitats and the coexistence with other species (McGill et al., 2006; Ackerly and Cornwell 2007; Adler et al., 2013). They are the objects (or targets, media) on which selection processes work, such as environmental filtering and interspecific competition, resulting in certain patterns of traits in local communities. Trait-based approaches aim at inferring community assembly processes from the patterns of traits within and between communities. A community with species possessing similar traits is usually inferred to result from environmental filtering, while coexisting species 
with different traits indicate interspecific competition or niche partitioning (Widenfalk et al., 2015, 2016). Soil ecologists have recently adopted the concept of functional traits to investigate belowground community structure and its association with the environment (Vandewalle et al., 2010; Pey et al., 2014; Moretti et al., 2017). Functional traits of soil invertebrates include morphological characters (e.g., body size, pigmentation and eye morphology), life history traits (e.g., reproductive mode and fitness), physiological traits (e.g., metabolic rate and desiccation resistance) and behavioral traits (e.g., dispersal mode) (Pey et al., 2014; Moretti et al., 2017). Using functional traits has been shown to be more powerful than using species identity for predicting the environmental associations of communities in soil (Makkonen et al., 2011; Bokhorst et al., 2012).

Collembola are among the few soil taxa for which trait databases are available (Vandewalle et al., 2010; Pey et al., 2014). Collembola traits, such as reproductive mode and morphological characters, are associated with the environment they live in (Makkonen et al., 2011; Bokhorst et al., 2012; Salmon and Ponge 2012; Salmon et al., 2014; Widenfalk et al., 2015). Spherical body shape, large body size, dark pigmentation and sexual reproduction are characteristic for species occurring in open habitats and at the soil surface, whereas small body size, lack of eye spots, pale color and asexual reproduction are typical traits of species inhabiting forests and living in soil (Salmon et al., 2014). That particular traits are associated with habitat characteristics suggest that community assembly processes are driven by selection processes (Vellend 2016), e.g. environmental filtering or interspecific competition (Widenfalk et al., 2015, 2016). In this thesis, I will focus on morphological traits because other trait categories were not studied for tropical Collembola. I will use the form of the abdomen, types of antennae, body length, presence and absence of empodial appendage, furca development, types of mouthparts, post-anttenal organ (PAO), pigmentation and presence or absence of scales.

\section{Fatty acid analysis as tool for soil food-web analysis}

Lipids play a vital role in animals both as storage compounds for providing energy (neutral lipid fatty acids, NLFAs) and as structural component of cell membranes (phospholipid fatty acids, PLFAs; Ruess et al., 2002). Biomarker FAs are synthesized only by microorganisms and plants, and transferred through food chains without or with little modification, allowing to infer links between basal resources and higher order consumers (Chamberlain et al., 2005; Ruess and Chamberlain, 2010). In soil food webs, it has been shown that fatty acids (FAs) are transferred from microorganisms to 
microbivorous invertebrates to higher order consumers (Ruess et al., 2002, 2005; Chamberlain et al., 2005) as well as from microorganisms and plants to detritivores and their predators (Pollierer et al., 2010). Thus, lipid profiles of consumers may serve as tool for soil food web diagnostics (Kühn et al., 2018).

Fatty acid analysis is a well-established tool for studying trophic interactions in many different ecosystems. FA methods were subsequently extended to higher trophic levels, including for example omnivorous Collembola (Ferlian et al., 2012; Pollierer et al., 2010). FA signatures can be used as dietary tracers due to the diverse array of specific FAs that originate from bacteria, fungi, algae and plants that animals are not capable to synthesize themselves (Traugott et al., 2013). In temperate forests, land-use and management practices have been shown to affect microbial community composition and the relative strengths of the fungal and bacterial energy channel (Pollierer et al., 2015). In this study, I used NLFAs of six soil animal taxa to investigate changes in the channeling of energy through soil food webs of tropical ecosystems.

\section{Investigating trophic position of and utilization of food resources by Collembola using stable isotope analysis}

Over the last two decades, stable isotope analysis has become the most commonly used tool to assess trophic niches of soil animals (Potapov et al., 2019). Two isotopic ratios, ${ }^{13} \mathrm{C} /{ }^{12} \mathrm{C}$ (i.e., $\delta^{13} \mathrm{C}$ values) and ${ }^{15} \mathrm{~N} /{ }^{14} \mathrm{~N}$ (i.e., $\delta^{15} \mathrm{~N}$ values), typically are used in food web studies. Trophic positions and length of trophic chains can be assessed using $\delta^{15} \mathrm{~N}$ values, whereas the range of $\delta^{13} \mathrm{C}$ values reflects the variability in the use of basal resources (Potapov et al., 2019). Stable isotope composition of consumers follows that in food resources, thus allowing to reveal potential diet switching with land-use change (Klarner et al., 2017; Krause et al., 2019; Susanti et al., 2019). Chahartaghi et al. (2005) investigated ${ }^{15} \mathrm{~N} /{ }^{14} \mathrm{~N}$ ratios of a major decomposer group, Collembola, to evaluate trophic relationship and determine feeding guilds. The results showed that trophic niches of Collembola species differ, indicating pronounced trophic niche differentiation and this likely contributes to Collembola species diversity.

In my studies, I used stable isotope analysis to investigate trophic positions and food resources of soil and litter Collembola to reveal how trophic positions and food resources of Collembola change after rainforest conversion into agricultural plantations, 


\section{Chapter 1}

such as rubber and oil palm, and explore patterns in trophic niche differentiation among tropical Collembola species.

\section{Methodology, hypotheses and objectives}

The studies of this thesis use several ecological methods to investigate the response of soil fauna to the conversion of rainforest into agricultural plantations (rubber and oil palm plantations). As a first general approach, I used fatty acid analysis to investigate changes in energy channeling in soil food webs for six soil animal taxa abundant in the study area, including two mesofauna decomposer taxa (Collembola and Oribatida), two macrofauna decomposer taxa (Diplopoda and Lumbricina), one mesofauna predator taxon (Mesostigmata) and one macrofauna predator taxon (Chilopoda). In the following I focused on Collembola as model taxonomic group. Collembola were identified to morphospecies level, which was additionally validated using molecular tools. In the first study I investigated changes in abundance, richness, functional composition of litter and soil Collembola using species and trait community composition. In the second study I used stable isotope analysis to investigate changes in food resources and trophic positions of litter and soil Collembola after conversion of rainforest into plantation systems.

The studies in this thesis formed part of the CRC990-EFForTS project, a DFG (Deutsche Forschungsgemeinschaft) funded interdisciplinary research project. Samples were taken in the framework of the 'Core Plot Design' of the CRC990-EFForTS consortium (Drescher et al., 2016). Samples from $50 \mathrm{~m} \times 50 \mathrm{~m}$ plots across four land-use systems have been studied including rainforest, jungle rubber, rubber plantation and oil palm plantation in two landscapes, Bukit Dua Belas and Harapan, in Jambi Province, Sumatra, Indonesia (Fig. 1). Soils in the Harapan region mainly comprise loamy Acrisols with low fertility, whereas in the Bukit Duabelas region the major soil type is clay Acrisol (Allen et al., 2015; Kotowska et al., 2015). In addition to the core plots, samples from riparian plots with either rainforest, rubber or oil palm plantations were studied, resulting in total 44 study plots with 4-8 replicates for each land-use system. Riparian plots were included in the project design to investigate the effect of seasonal flooding on ecosystem parameters (riparian plots stay under the water each year from November until April during the 'rainy season'). In each studied plot, three $16 \times 16 \mathrm{~cm}$ soil samples were taken and divided into litter and soil $(5 \mathrm{~cm}$ in depth). Soil animals were extracted using heat 


\section{Chapter 1}

(Kempson et al., 1963) and transferred into $70 \%$ ethanol and transported to the University of Goettingen for detailed taxonomic identification and further analyses.

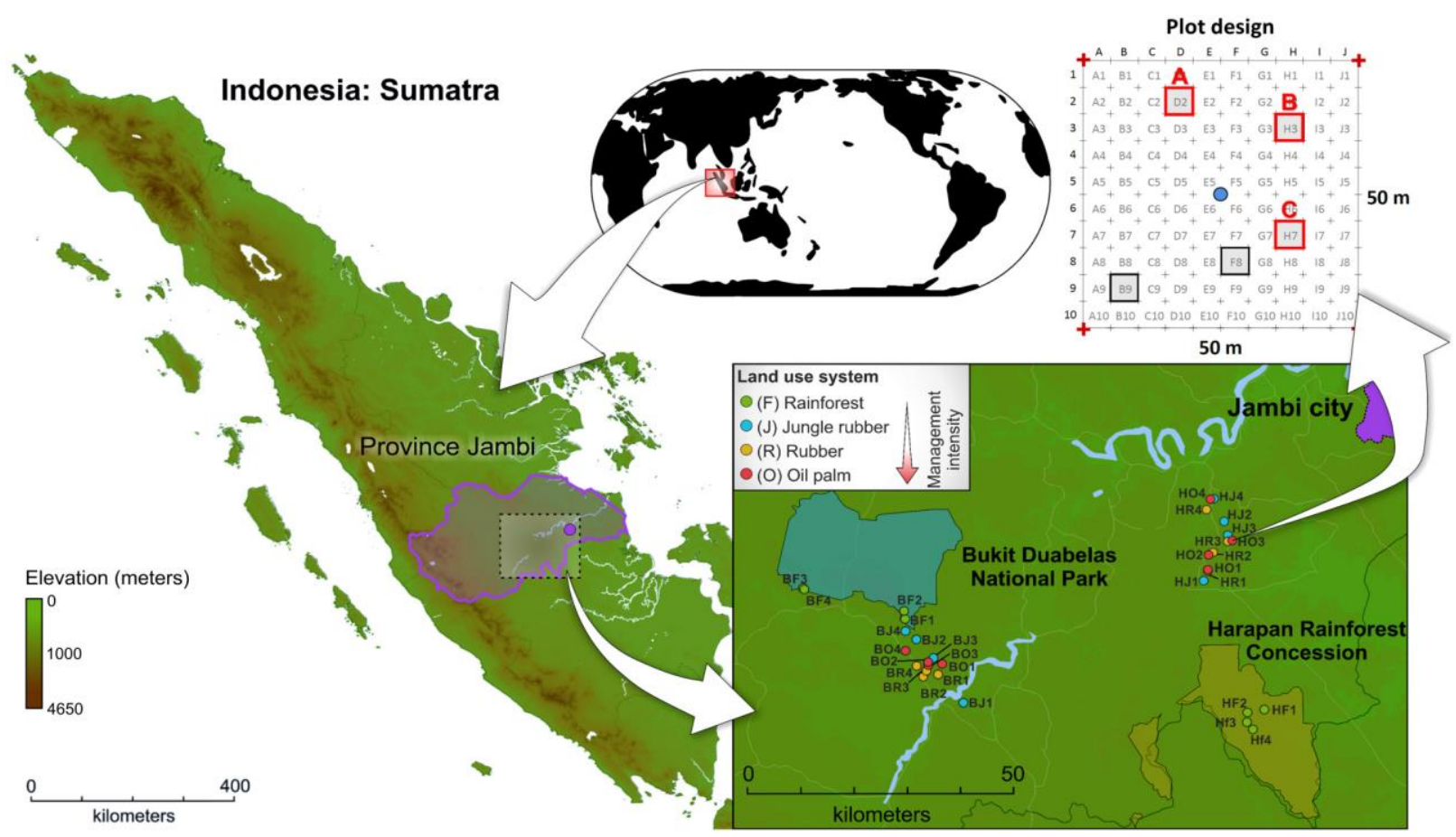

Figure 1. Location of EFForTS study sites in Sumatra, Jambi Province. The Core Plot Design is based on two landscapes near Bukit Duabelas National Park and Harapan Rainforest (plots marked with grey squares) (from Drescher et al., 2016).

In this $\mathrm{PhD}$ thesis I explored the effects of rainforest conversion to agricultural plantations (rubber and oil palm) in Jambi province (Sumatra, Indonesia) in 2013, 2016 and 2017 focusing on trophic interactions in the belowground food web and community composition of Collembola.

The following overall hypotheses were investigated:

(1) Soil food webs change in response to the conversion of rainforest into agricultural plantations, with the bacterial energy channel being more important in rainforest and the plant energy channel being more important in plantations.

(2) Density, species diversity, and functional diversity are higher in rainforest than in plantations system, with species and trait community composition differing between plantation systems. 


\section{Chapter 1}

(3) Trophic niche differentiation among Collembola species is more pronounced in rainforest than in plantation systems with Collembola in plantations shifting their trophic niches towards herbivory.

(4) Dry season has lower Collembola density and more pronounced differences in community composition among land-use systems.

To answer the hypotheses, the thesis contains the following objectives, corresponding to the four chapters:

(1) Conversion of rainforest into rubber and oil palm plantations alters energy channels in soil food webs (Chapter 2).

In this chapter, I investigate community-level neutral lipid fatty acid profiles in dominant soil fauna taxa to track energy channels in rainforest, rubber and oil palm plantations in Sumatra, Indonesia. The material was collected in 2017. Abundant macrofauna including Araneae, Chilopoda and Diplopoda contained relatively high amounts of plant and fungal biomarker fatty acids (FAs). Lumbricina had the lowest amount of plant, but the highest amount of animal-synthesized C20 polyunsaturated FAs compared to other soil taxa. Mesofauna detritivores (Collembola and Oribatida) contained relatively high amounts of algal biomarker FAs. The differences in FA profiles between taxa were evident if data were analysed across land-use systems, suggesting that soil fauna of different size (macro- and mesofauna) are associated with different energy channels. Despite that, rainforest conversion changed the biomarker FA composition of soil fauna at the community level. Conversion of rainforest into oil palm plantations enhanced the plant energy channel in soil food webs and reduced the bacterial energy channel; conversion into rubber plantations reduced the AMF-based energy channel. The changes in energy distribution within soil food webs may have significant implications for the functioning of tropical ecosystems and their response to environmental changes.

(2) Conversion of rainforest to rubber and oil palm plantations affects the functional composition of litter and soil Collembola (Chapter 3).

This chapter investigates the response of Collembola to the conversion of rainforest into rubber and oil palm plantations. Collembola from litter and soil layers were extracted in 2013 and 2016. In the litter layer, the abundance and 
species richness generally declined by $25-37 \%$ and $30-40 \%$, respectively, in plantations compared to rainforest. By contrast, in the soil layer, density, species richness and trait diversity of Collembola changed little. Species and trait composition of Collembola communities in the litter and soil layer depended on land use. Water content and $\mathrm{pH}$ both in the litter and soil layer were the main factors explaining the differences in species and trait community composition followed by the density of meso- and macrofauna predators. Dominating Collembola species in rainforest and jungle rubber were mostly characterized by small size, absence of furca and absence or intense pigmentation, while larger species with long furca and diffuse or patterned coloration were relatively more abundant in plantations. Overall, the land-use change altered Collembola communities in particular in litter but less in soil. Certain dominant species were found across all land-use systems, reflecting their ability to adapt to environmental changes, such as changes in $\mathrm{pH}$, water content and top-down control. The decline in diversity and abundance of litter-dwelling Collembola calls for management practices mitigating the negative effect of the deterioration of the litter layer in oil palm and rubber plantations.

(3) Trophic niche differentiation of and utilization of food resources by Collembola is altered by the conversion of rainforest into plantations (Chapter 4).

In this chapter, stable isotope analysis $\left({ }^{13} \mathrm{C}\right.$ and $\left.{ }^{15} \mathrm{~N}\right)$ was used to investigate changes in the trophic structure and use of food resources by Collembola with land-use change. Isotopic composition of Collembola from 32 sites representing four land-use systems were analyzed (rainforest, rubber agroforest, and rubber and oil palm monoculture plantations). Across Collembola species $\Delta^{13} \mathrm{C}$ values were highest in rainforest, suggesting predominant consumption of saprotrophic microorganisms associated with decomposed litter materials in this system. Lower $\Delta{ }^{13} \mathrm{C}$ values, but high $\Delta{ }^{13} \mathrm{C}$ variation in Collembola in oil palm plantations indicated that Collembola shifted towards herbivory and used more variable resources in this system. Small range in $\Delta^{15} \mathrm{~N}$ values in Collembola species in monoculture plantations in comparison to rainforest indicated that the conversion of rainforest into 


\section{Chapter 1}

plantations is associated with simplification in the trophic structure of Collembola communities. This was confirmed by generally lower isotopic niche differentiation among species in plantations. Across the studied ecosystems, atmobiotic species (Symphypleona and Paronellidae) occupied the lowest, whereas euedaphic Collembola species occupied the highest trophic position, resembling patterns in temperate forests. Some species of Paronellidae in rainforest and jungle rubber had $\Delta^{15} \mathrm{~N}$ values below those of leaf litter suggesting algivory (Salina sp.1, Callyntrura sp.1 and Lepidonella sp.1), while Pseudosinella sp.1 had the highest $\Delta^{15} \mathrm{~N}$ values in most of the land-use systems suggesting that this species at least in part lives as predator or scavenger. Overall, the results suggest that rainforest conversion into plantation systems is associated with marked shifts in the structure of trophic niches in soil and litter Collembola with potential consequences for ecosystem functioning and food-web stability.

(4) Environmental factors explain seasonal shifts in Collembola community composition in different land-use system (Chapter 5).

In this chapter, I investigated effect of seasonal fluctuations and corresponding changes in environmental factors on the community composition of litter and soil Collembola. Collembola density in the litter layer was at a maximum in the wet season, whereas in the soil layer the density of Collembola strongly decreased in the wet season. Euedaphic species were better adapted to seasonal fluctuations and dominanted in all land-use systems. Collembola community composition in the different land-use systems was affected by seasonal fluctuations with differences being more pronounced in the litter than in soil layer. Differences in community composition in litter and soil of all land-use system were more pronounced in the dry season. Water content and $\mathrm{pH}$ were identified as main factors related to the differences in species composition of Collembola both in litter and soil layers with seasonal changes, followed by the density of bacterial (Gram-positive and Gram-negative bacteria), fungal and arbuscular mycorrhizal fungal density. In addition, C/N ratio was identified as important factor driving Collembola community composition in the soil layer of rainforest. 


\section{CHAPTER 2}

CONVERSION OF RAINFOREST TO OIL PALM AND RUBBER PLANTATIONS ALTERS ENERGY CHANNELS IN SOIL FOOD WEBS

Winda Ika Susanti, Melanie M Pollierer, Rahayu Widyastuti, Stefan Scheu, Anton Potapov
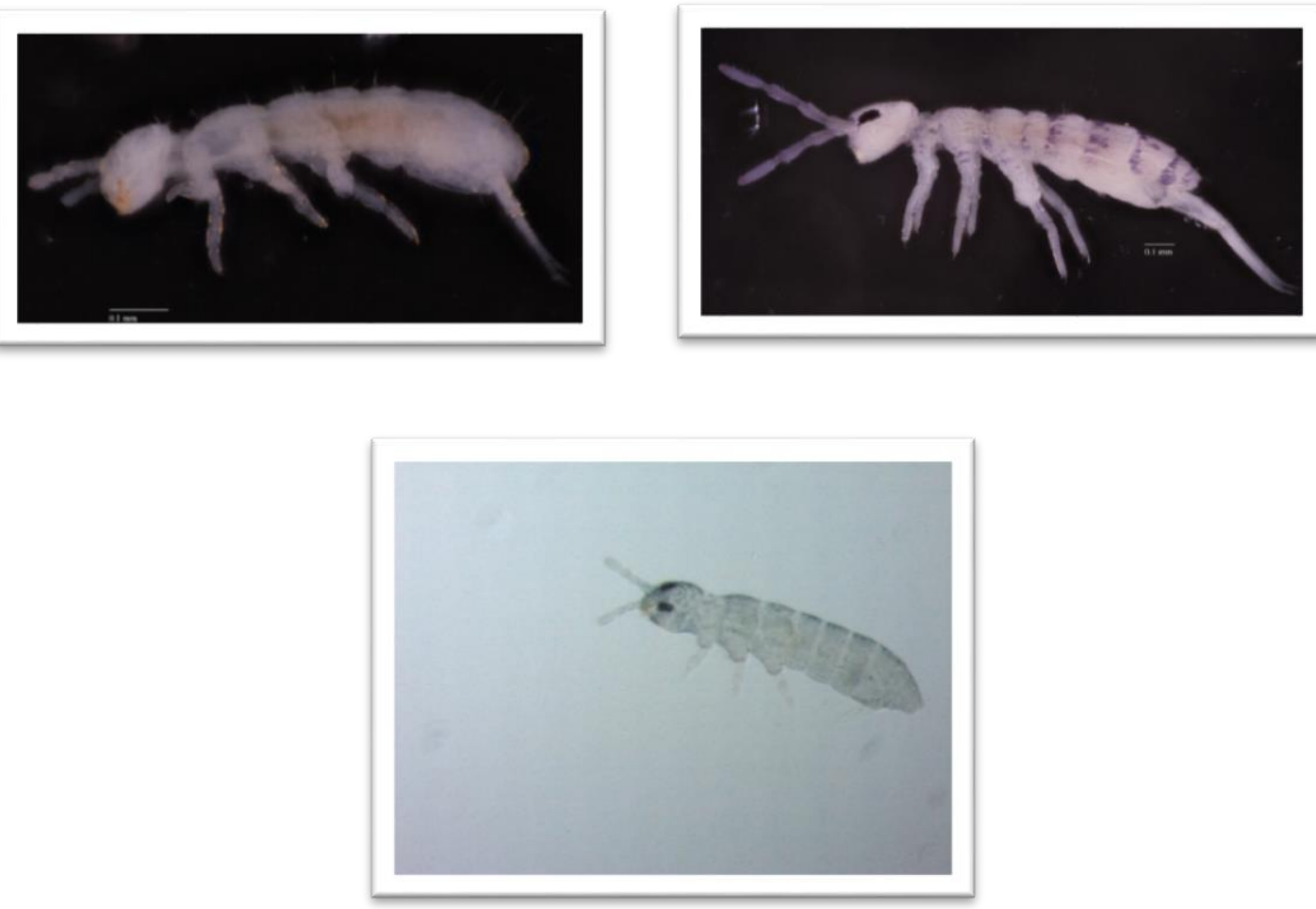

Photos by Winda Ika Susanti 


\title{
CONVERSION OF RAINFOREST TO OIL PALM AND RUBBER PLANTATIONS ALTERS ENERGY CHANNELS IN SOIL FOOD WEBS
}

\begin{abstract}
In the last decades lowland tropical rainforest has been converted in large into plantation systems. Despite the evident changes above ground, the effect of rainforest conversion on the channeling of energy in soil food webs was not studied. Here we investigated community-level neutral lipid fatty acid profiles in dominant soil fauna to track energy channels in rainforest, rubber and oil palm plantations in Sumatra, Indonesia. Abundant macrofauna including Araneae, Chilopoda and Diplopoda contained high amounts of plant and fungal biomarker fatty acids (FAs). Lumbricina had the lowest amount of plant, but the highest amount of animal-synthesized C20 polyunsaturated FAs as compared to other soil taxa. Mesofauna detritivores (Collembola and Oribatida) contained high amounts of algal biomarker FAs. The differences in FA profiles between taxa were evident if data were analysed across land-use systems, suggesting that soil fauna of different size (macro- and mesofauna) are associated with different energy channels. Despite that, rainforest conversion changed the biomarker FA composition of soil fauna at the community level. Conversion of rainforest into oil palm plantations enhanced the plant energy channel in soil food webs and reduced the bacterial energy channel; conversion into rubber plantations reduced the AMF-based energy channel. The changes in energy distribution within soil food webs may have significant implications for the functioning of tropical ecosystems and their response to environmental changes. At present, these responses are hard to predict considering the poor knowledge on structure and functioning of tropical soil food webs.
\end{abstract}

Keywords: soil fauna, biomarker, fatty acids, land-use change, macrofauna, mesofauna

\section{Introduction}

Indonesia is a hotspot of biodiversity on Earth and at the same time a top world producer of agricultural products, such as oil palm (Fitzherbert et al., 2008; Koh and Ghazoul, 2010) and rubber (Marimin et al., 2014). Extension of agriculture in Indonesia 
was associated with deforestation which increased strongly in the last 20 years and this is predicted to continue (Koh and Ghazoul, 2010; Gatto et al., 2015). Since the 1980s, after the transmigration program, large parts of rainforest in Jambi province, Sumatra, have been converted to oil palm (16\% of total area) and rubber plantations (12\%; Gatto et al., 2015). Thus, Sumatra represents an ideal model region to investigate the effect of rainforest conversion on biodiversity and ecosystem functioning at local and regional scale in Southeast Asia (Clough et al., 2016; Drescher et al., 2016).

Conversion of tropical rainforests into plantation systems is associated with changes in ecological niches of species and ultimately with the loss of species, and thereby with changes in ecosystem functioning (Fitzherbert et al., 2008; Gilbert, 2012; Barnes et al., 2014; Clough et al., 2016). Rainforest conversion strongly affects environmental processes, including soil organic carbon pools and soil erosion (Guillaume et al., 2015), primary production of trees (Kotowska et al., 2015), carbon dioxide and methane fluxes (Hassler et al., 2015), as well as nitrogen cycling and soil fertility (Allen et al., 2015). However, the effect of conversion of rainforest into plantations on belowground organisms is less well studied. It has been shown that rainforest conversion strongly affects biomass, vitality and mycorrhizal colonization of roots (Sahner et al., 2015), biodiversity and abundance of microorganisms (Krashevska et al., 2015; Schneider et al., 2015) and trophic-guild composition of litter-dwelling fauna (Barnes et al., 2014; Klarner et al., 2017). However, the effect of rainforest conversion on food resources of soil fauna and the relative importance of energy channels of soil food webs (Moore et al., 2004) is little understood. This is unfortunate, as the way energy is channelled through soil food webs is a major determinant of their stability (Ruiter, Neutel and Moore, 1995; Moore and De Ruiter, 2012).

Rainforest conversion is likely to alter the flux of energy through soil food webs. For instance, nutrient inputs, changes in soil $\mathrm{pH}$, as well as physical disturbances caused by management practices may shift fungal- and bacterial-based energy channels in soils (Rousk et al., 2010). In temperate regions more fertile and productive ecosystems foster the bacterial-based energy channel, while less fertile ecosystems foster the fungal-based energy channel (Wardle et al., 2004). Previous studies showed that conversion of rainforest into oil palm plantations results in a decreased amount of specific bacterial biomarker PLFAs in the litter (Krashevska et al., 2015) and causes a diet shift of generalist predators towards more herbivore prey (Klarner et al., 2017). However, the resulting changes in energy channels of the entire soil food web have not been explored. 
Soil organisms interact in complex ways in soil with their activity regulating ecosystem processes and delivering ecosystem services (Lavelle et al., 2006). Food web models allow calculating energy and nutrient fluxes based on consumer - resource interactions and the way how energy and nutrients are channelled through soil microbial and animal communities (De Ruiter et al., 1993). This channelling has been shown to be altered significantly with land-use intensification (Wagg et al., 2014). Building on these knowledge we explored changes in the channelling of energy through soil food webs after conversion of rainforest into plantation systems of rubber and oil palm.

Lipids play a vital role in animals both as storage compounds, for providing energy (neutral lipid fatty acids, NLFAs) and as structural component of cell membranes (phospholipid fatty acids, PLFAs; Ruess et al., 2002). Biomarker FAs are synthesized only by microorganisms and plants and transferred through food chains without modification, allowing to infer links between basal resources and higher order consumers (Chamberlain et al., 2005; Ruess and Chamberlain, 2010). For soil food web, it has been shown that fatty acids (FAs) are transferred from microorganisms to microbivorous invertebrates to higher order consumers (Ruess et al., 2002, 2005; Chamberlain et al., 2005) as well as from microorganisms and plants to detritivores and their predators (Pollierer, Scheu and Haubert, 2010). Thus, lipid profiles of consumers may serve as tool for soil food web diagnostics (Kühn et al., 2018). To-date, however, virtually no studies investigated lipid profiles of soil fauna including both meso- and macrofauna in the same community.

Here we used lipid profiles of soil fauna to track how energy channels in soil food webs are changing due to rainforest conversion into two major agricultural systems, i.e. rubber and oil palm plantations, in Sumatra, Indonesia. We hypothesized that (1) different groups of meso- and macrofauna are linked to different basal resources with mesofauna detritivores, i.e. Collembola and Oribatida, being closely associated with the fungal energy channel, and macrofauna detritivores such as Lumbricina and Diplopoda being more closely associated with the bacterial and plant energy channel. Further, based on results of previous studies (Krashevska et al., 2015; Klarner et al., 2017), which indicated reduced feeding on bacteria and increased feeding on plants with conversion of rainforest into oil palm plantations, we hypothesized (2) that rainforest conversion strengthens the plant-based energy channel and reduces the bacterial energy channel in soil food webs of plantation systems. 


\section{Chapter 2}

\section{Material and methods}

\section{Study sites}

Soil and litter samples were taken in lowland rainforest, rubber (Hevea brasiliansis) plantations and oil palm (Elaeis guineensis) plantations, located in Jambi province, southwest Sumatra, Indonesia. Jambi province stretches from the Barisan mountain range in the west across extensive lowlands towards the southern Malacca Strait in the east. The climate is tropical and humid with rainy seasons from March to December, and a dryer period during July and August (Drescher et al., 2016). Study sites were located at similar altitude varying between 50 and $100 \mathrm{~m}$ a.s.l. in Harapan landscape; each system was replicated four times (see Drescher et al., 2016 for more details). Rainforest sites used as reference comprised secondary rainforest close to natural condition that underwent selective logging some 20-30 years ago. Rubber and oil palm plantations were intensively managed monocultures of an average age of 6 to 16 and 8 to 15 years, respectively (Drescher et al., 2016). Soils at the study sites were loam acrisols of low fertility (Allen et al., 2015).

Management practices in these smallholder monoculture plantations are described in detail by Allen et al., (2015). In the loam Acrisol soil, oil palm plantations were established after clearing and burning the previous jungle rubber whereas the rubber plantations were established from previously logged forest. Oil palms are fertilized once in the rainy season and once in the dry season. The most commonly used fertilizers are NPK complete fertilizer (i.e. Phonska and Mahkota), potassium chloride $(\mathrm{KCl})$ and urea $\left(\mathrm{CO}\left(\mathrm{NH}_{2}\right)_{2}\right)$. Both manual and chemical weeding took place throughout the year at the rubber and oil palm plantations. The most commonly used herbicides were Gramoxone and Roundup; these were applied at an average rate of 2 to $5 \mathrm{~L}_{\text {herbicide }} \mathrm{ha}^{-1}$ year $^{-1}$ (Allen et al., 2015; Kotowska et al., 2015; Clough et al., 2016).

The description of major soil properties refers to the study by Krashevska et al. (2015) on the same field sites. Soil water content decreased between transformation systems in the order rainforest (39.94 $\pm 7.46 \%)$, rubber $(38.22 \pm 5.85 \%)$, and oil palm plantation $(30.29 \pm 6.47 \%)$. Soil $\mathrm{pH}$ was higher in converted systems and decreased in the order oil palm $(4.97 \pm 0.34)$, rubber $(4.44 \pm 0.11)$, and rainforest $(3.95 \pm 0.17)$. Total soil C concentration was higher in rainforest $(4.25 \pm 1.26 \%)$, but was similar in rubber $(2.35 \pm 0.73 \%)$ and oil palm plantation $(2.57 \pm 1.05 \%)$. Similarly, total soil $\mathrm{N}$ concentration was higher in rainforest $(0.28 \pm 0.06 \%)$, but was similar in rubber $(0.19 \pm$ 
$0.05 \%)$ and oil palm plantation $(0.18 \pm 0.04 \%)$. Soil C-to-N ratio was similar in rainforest $(14.95 \pm 1.56)$ and oil palm $(14.31 \pm 4.50)$, but lower in rubber plantation $(11.64 \pm 1.23)$.

\section{Sampling}

Samples were taken in September 2017 from three 5 x $5 \mathrm{~m}$ subplots within $50 \mathrm{x}$ $50 \mathrm{~m}$ plots with a minimum distance of $200 \mathrm{~m}$ between plots established at each study site (Drescher et al., 2016). Each sample measured 16 x $16 \mathrm{~cm}$ and included the litter layer and underlying top soil to a depth of $5 \mathrm{~cm}$. Since only few soil fauna were collected in oil palm plantation we performed an additional non-quantitative sampling of litter and soil in this system. Large soil fauna (> $2 \mathrm{~mm}$ in body length; macrofauna) were collected by hand after sieving the litter through $2 \mathrm{~cm}$ mesh; small soil fauna $(<2 \mathrm{~mm}$; mesofauna) were extracted by heat (Kempson, Lloyd and Gheraldi, 1963) during three days in water. Every day the extracted mesofauna species were picked manually from the water surface and identified to a family/order level under a dissecting microscope. To track the community-level changes fauna was collected non-selectively and then groups, dominated by biomass, were analyzed. The collection comprised six key groups: Collembola (families Entomobryidae and Paronellidae, and order Symphypleona), Oribatida (families Galumnidae, Otocepheidae, Galumnellidae, Scheloribatidae, Haplozetidae, Zetordestidae, and Lohmanniidae), Diplopoda (families Polydesmidae and Julidae), Araneae, Lumbricina, and Chilopoda (families Geophilomorpha and Scolopendromorpha; see Supplementary Table S1 for the full list of taxa analyzed). To get enough material for lipid analysis, small fauna from different subplots and layers were bulked (in few cases animals from two plots within the same system were combined); one sample consisted of 1-54 specimens of 0.5-2 mg fresh weight. After identification animals were immediately transferred into pure methanol and stored at $-20^{\circ} \mathrm{C}$ before fatty acid extraction (Zieger and Scheu, 2018). In total, 86 samples of soil fauna were analysed. Data on fatty acid composition of all samples are available from Dryad Digital Repository (https://doi.org/10.5061/dryad.d6n77r3).

\section{Analysis of fatty acids and biomarker assignment}

NLFAs were extracted as described in Ruess et al. (2005). In brief, animals were placed in $5 \mathrm{ml}$ single phase extraction solvent (chloroform, methanol, $0.05 \mathrm{M}$ phosphate buffer at a ratio of 1:2:0.8; $\mathrm{pH} 7.4$ ) and NLFAs were extracted overnight on a shaker. The 
solvent was then transferred to new tubes and the extraction was repeated by shaking for 1-2 $\mathrm{h}$ with an additional $2.5 \mathrm{ml}$ of extraction solvent. Extraction solvents of both steps were combined, $0.8 \mathrm{ml}$ of distilled water and $0.8 \mathrm{ml}$ of $\mathrm{CHCl}_{3}$ were added, and samples were centrifuged at $7^{\circ} \mathrm{C}$ in a multi centrifuge $(1500 \mathrm{rpm})$ for $5 \mathrm{~min}$. Then, samples were allowed to stand to separate phases. The top two phases were removed and the chloroform fraction was transferred to a silica gel $(\mathrm{SiOH})$ column $(0.5 \mathrm{~g}$, mesh size 100-200 $\mu \mathrm{m})$. Lipids were eluted with $5 \mathrm{ml}$ of chloroform and the solvent was reduced by evaporation in a vacuum centrifuge. NLFAs were saponified and methylated following the procedure given for the Sherlock Microbial Identification System (MIDI Inc., Newark, Del). For saponification a solution of sodium hydroxide/methanol was used and the samples incubated at $100^{\circ} \mathrm{C}$ for $30 \mathrm{~min}$ followed by acid methanolysis in $\mathrm{HCl}$-methanol at $80^{\circ} \mathrm{C}$ for $10 \mathrm{~min}$. The resulting fatty acid methyl esters (FAMEs) were stored at $-20^{\circ}$ until analysis.

FAMEs were transferred into vials, capped and analyzed by gas chromatography (Clarus 500, Perkin Elmer, Waltham, USA). Helium was used as carrier gas and NLFAs were identified by a flame ionization detector (capillary column: $30 \mathrm{~m} \times 0.32 \mathrm{~mm}$ i.d., $0.25 \mathrm{~mm}$ film thickness; PE-5, Perkin Elmer). The temperature program started with a temperature of $60^{\circ} \mathrm{C}$ (held for $1 \mathrm{~min}$ ) and increased by $30^{\circ} \mathrm{C}$ per min to $160^{\circ} \mathrm{C}$ followed by $3^{\circ} \mathrm{C}$ per min to $260^{\circ} \mathrm{C}$. The injection temperature was $250^{\circ} \mathrm{C}$ and helium was used as carrier gas. On the basis of retention time and comparison with standard mixtures, 37 different FAMEs ranging from $\mathrm{C} 11$ to $\mathrm{C} 24$ and 26 different BAMEs ranging from $\mathrm{C} 11$ to C20 were identified. Hexadecadienoic acid methyl ester and methyl-hexadecatrienoate were used as algal biomarkers (SigmaeAldrich, St Louis, USA). Biomarker FAs for different taxa were assigned according to published studies (Table 2.1). To illustrate differences in proportions of biomarker FAs between taxa and systems, we used an array of biomarker FA-based indices: Arbuscular mycorrhizal fungi (AMF) biomarker (16:1 $\omega 5)$, sum of plant biomarkers $(18: 3 \omega 6,9,12 ; 18: 1 \omega 9 ; 22: 0$ and $24: 0)$, fungal biomarker $(18: 2 \omega 6,9)$, sum of algae biomarkers $(16: 2 \omega 6,9$ and 16:3 $166,9,12)$, sum of animal-synthesized PUFAs $(20: 4 \omega 6,9,12,15$ and 20:5 $\omega 3,6,9,12,15)$, non-specific

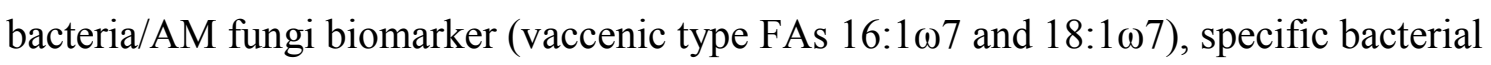
biomarkers (sum of all Gram+ bacteria biomarkers i15:0, a15:0, i16:0, i17:0 and Grambacteria biomarkers cy17:0, cy 19:0, 2-OH 12:0, 3-OH 12:0, 2-OH 14:0, 3-OH 14:0), ratio between fungal biomarker and plant biomarkers, and ratio between fungal biomarker and specific bacterial biomarker FAs. 
Table 2.1. List of fatty acids (FAs) used as biomarkers.

\begin{tabular}{|c|c|c|}
\hline FA & Resource & References \\
\hline $16: 1 \omega 5$ & $\begin{array}{l}\text { Arbuscular mycorrhizal } \\
\text { fungi (AMF) }\end{array}$ & Olsson, 1999; Madan et al., 2002 \\
\hline $16: 2 \omega 6,9$ & Green algae & Buse, Ruess and Filser, 2013 \\
\hline $16: 3 \omega 3,6,9$ & Green algae & Buse, Ruess and Filser, 2013 \\
\hline $20: 4 \omega 6,9,12,15$ & Animal-synthesized* & Chen et al., 2001; Chamberlain et al., 2005 \\
\hline $20: 5 \omega 3,6,9,12,15$ & Animal-synthesized* & Chamberlain et al., 2005 \\
\hline $18: 2 \omega 6,9$ & Fungi & $\begin{array}{l}\text { Frostegard and Baath, 1996; Ruess et al., 2005; } \\
\text { Haubert et al., 2006; Ruess and Chamberlain, } 2010\end{array}$ \\
\hline $16: 1 \omega 7$ & $\begin{array}{l}\text { General/widespread } \\
\text { bacteria }\end{array}$ & $\begin{array}{l}\text { Welch, 1991; Zelles, 1999; Ruess et al., 2005; } \\
\text { Haubert et al., 2006; Ruess and Chamberlain, } 2010\end{array}$ \\
\hline $18: 1 \omega 7$ & $\begin{array}{l}\text { General/widespread } \\
\text { bacteria }\end{array}$ & Welch, 1991; Zelles, 1999 \\
\hline i15:0 & Gram+ bacteria & Zelles, 1999; Haubert et al., 2006 \\
\hline a15:0 & Gram+bacteria & Zelles, 1999; Haubert et al., 2006 \\
\hline i16:0 & Gram+bacteria & Zelles, 1999; Haubert et al., 2006 \\
\hline i17:0 & Gram+bacteria & Zelles, 1999; Haubert et al., 2006 \\
\hline $2-\mathrm{OH} \mathrm{12:0}$ & Gram- bacteria & Wakeham et al., 2003; Lee et al., 2004 \\
\hline 3-OH 12:0 & Gram- bacteria & Wakeham et al., 2003; Lee et al., 2004 \\
\hline $2-\mathrm{OH} \mathrm{14:0}$ & Gram- bacteria & Wakeham et al., 2003; Lee et al., 2004 \\
\hline $3-\mathrm{OH} \mathrm{14:0}$ & Gram- bacteria & Wakeham et al., 2003; Lee et al., 2004 \\
\hline cy17:0 & Gram- bacteria & Zelles, 1999 \\
\hline 2-OH 16:0 & Gram- bacteria & Wakeham et al., 2003; Lee et al., 2004 \\
\hline cy19:0 & Gram- bacteria & Zelles, 1999 \\
\hline $18: 3 \omega 6,9,12$ & Plants & Millar, Smith and Kunst, 2000; Ruess et al., 2007 \\
\hline $18: 1 \omega 9$ & Plants & $\begin{array}{l}\text { Harwood and Russell, 1984; Ruess et al., } 2005 \text {; } \\
\text { Ruess } \text { et al., } 2007\end{array}$ \\
\hline $22: 0$ & Plants & Zelles, 1999; Ruess et al., 2007 \\
\hline $24: 0$ & Plants & Zelles, 1999; Ruess et al., 2007 \\
\hline
\end{tabular}

*Animal-synthesized C20 polyunsaturated FAs cannot be used as biomarkers for animal consumption 


\section{Statistical analysis}

Statistical computations were done in R version 3.4.0 (R Core Team, 2017) with R Studio interface (R studio Inc.). To normalize the data across different taxa and sites, we analyzed proportions of total (\%) of individual NLFAs instead of the initial peak areas. We first calculated mean proportions for each NLFA for each group on each plot. In cases where more than one sample of the same group was analyzed per plot, we calculated mean proportions across these samples. We used the mean values for each taxon on each plot as replicates in statistical analyses (Supplementary Material: Table S2). Proportional data were arcsine- and logit-transformed. After visual inspection of distributions for individual NLFAs, arcsine transformation was chosen for further statistical analyses, as it was more close to normal.

To analyze the effect of taxonomic group on individual biomarker FAs and NLFA indices in soil fauna we used a subset of dominating groups found on at least four plots across at least two ecosystems, which were classified as macrofauna predators (Araneae and Chilopoda), macrofauna detritivores (Diplopoda and Lumbricina), or mesofauna detritivores (Collembola and Oribatida). Since different groups dominated different ecosystems and plots, we were not able to run a reliable full-design model with both taxonomic group and ecosystem type as fixed factors. Thus, we built a set of linear mixedeffects models with individual biomarker FAs as response variables and ecosystem (rainforest, rubber or oil palm) as random effect using the lme4 package (Bates et al., 2015). Significance of the overall effect was evaluated using the lmerTest package (Kuznetsova et al., 2016); significant differences between groups were tested using general linear hypothesis Tukey test in the multcomp package (Hothorn et al., 2008). Vice versa, to analyze the effect of ecosystem on individual biomarker FAs in soil fauna, we used a set of linear mixed-effects models with taxonomic group as random effect. Taxonomic group was used as random effect since we were interested in shifts of community food resources irrespective of community taxonomic composition. In addition, we analyzed shifts in NLFA composition between ecosystems using linear discriminant analysis (LDA) and multivariate analysis of variance (MANOVA) based on seven NLFA indices in vegan and MASS packages. Data are presented as means $\pm 1 \mathrm{SD}$; $p<0.05$ was used as the level of significance. 


\section{Chapter 2}

\section{Results}

\section{Food resources of different groups of soil fauna}

Twenty-three biomarker FAs were grouped into seven categories representing different basal resources and two indices were calculated: plant-to-fungi and fungi-to-

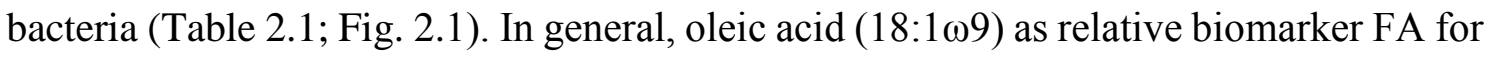
plants and linoleic acid $(18: 2 \omega 6,9)$ as relative biomarker for fungi were the most common fatty acids in soil fauna across ecosystems and taxa (Appendix Table 2.1). Groups of macrofauna (Araneae, Chilopoda, and Diplopoda) contained about twice higher proportions of fungal and plant biomarker FAs (Fig. 2.1) as compared to mesofauna groups (Collembola and Oribatida). Mesofauna detritivores (Collembola and Oribatida) contained four times higher proportions of algal biomarker FAs as compared to macrofauna groups. Collembola contained the highest proportion of algal biomarker, and the lowest proportion of fungal biomarker FAs across groups. This resulted in the highest ratio of plant-to-fungi and the lowest ratio of fungi-to-bacteria in Collembola. The concentration of Gram- bacteria biomarker FAs varied among groups, but tended to be higher in mesofauna than macrofauna; Oribatida contained the highest proportion of the Gram- bacteria biomarker FA 2-OH 14:0 and Collembola contained the highest proportion of the Gram- bacteria biomarker FA cy 17:0 (Appendix Table 2.1). Lumbricina contained the lowest proportion of plant biomarker FAs, but the highest proportion of fungal biomarker FAs and had by far the lowest ratio of plant-to-fungi biomarker FAs. Animal-synthesized (C20 polyunsaturated) FAs were four times higher in predators than in most detritivores except for Lumbricina that contained four times higher proportion of animal-synthesized FAs than both predatory groups (Araneae and Chilopoda). The proportion of the AMF and non-specific bacteria biomarker FAs did not differ significantly between taxonomic groups, but the latter were about twice lower in mesofauna than in macrofauna (Fig. 2.1). 


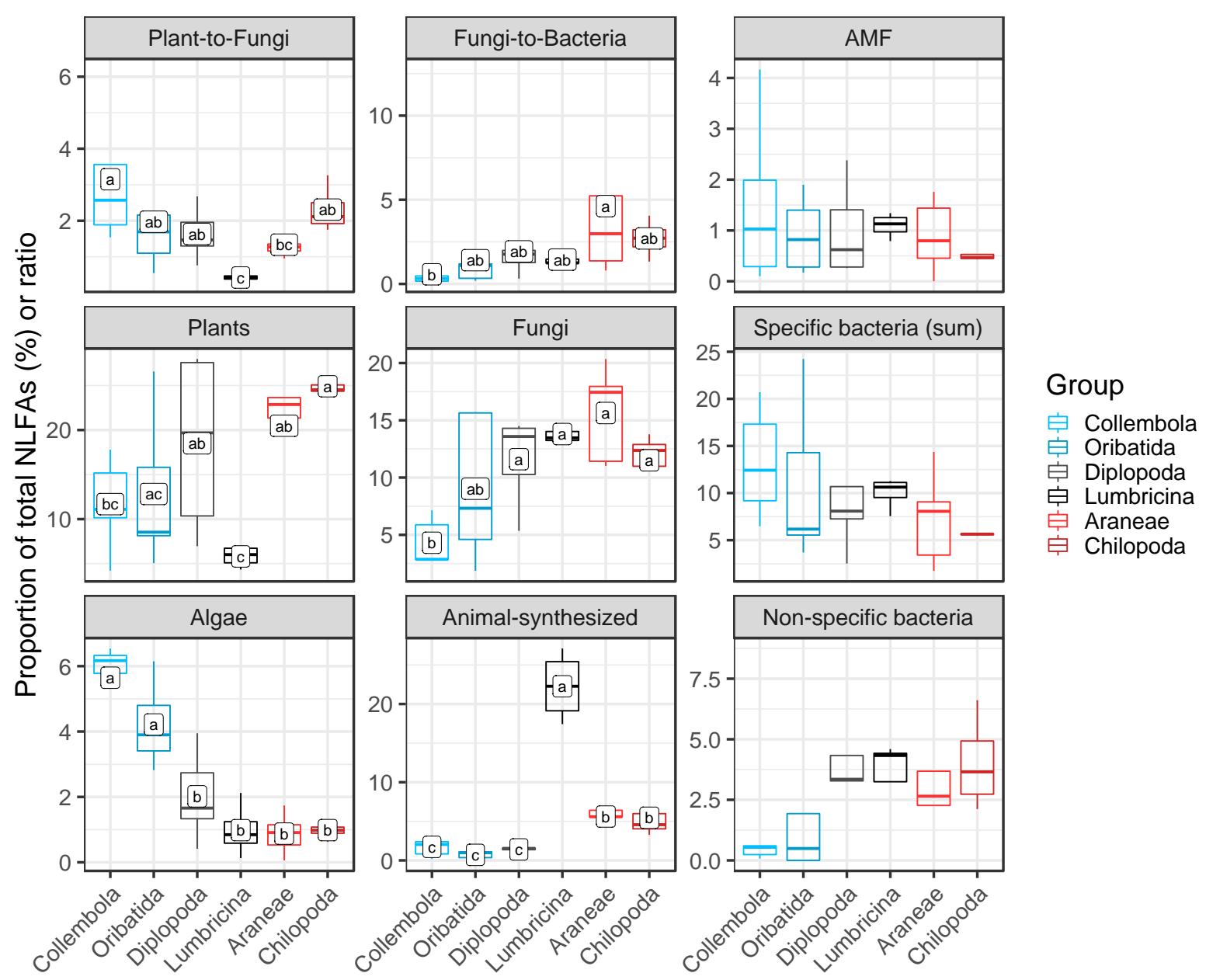

Figure 2.1. Biomarker fatty acid-based indices in dominating soil fauna across the studied ecosystems. All indices are given as proportion of total biomarker FAs except for plant-to-fungi and fungi-to-bacteria biomarker fatty acid ratios (see methods). Taxa include two groups of detritivore mesofauna (Collembola and Oribatida, blue), two groups of detritivore macrofauna (Diplopoda and Lumbricina, grey) and two predatory groups (Araneae and Chilopoda, red). Individual biomarker FAs with the same (or absent) letter are not significantly different according to Tukey's honestly significant difference test $(\mathrm{P}>0.05)$.

\section{Effect of land-use change on food resources of soil fauna}

Land-use affected the NLFA indices-based profiles, if all groups of soil fauna were analyzed together (MANOVA: $\mathrm{F}_{2,14}=1.82, \mathrm{p}=0.0469$; Fig. 2.2). The first axis of LDA, explaining $63 \%$ of distinction between land-use systems, was related to specific and non-specific bacterial biomarker FAs with the proportion of the former being higher in animals from rainforest and the latter being higher in animals from plantation systems (Appendix Table 2.2). AMF biomarker NLFA was positively associated with animal taxa 
in rainforest and negatively with animal taxa in plantation systems, especially in rubber. Plant and algae biomarker FAs were negatively intercorrelated, with the proportion of the former being higher in animals from rainforest and oil palm plantations, and the latter being higher in animals from rubber plantations. The proportion of the fungal biomarker FA was lower in animals from oil palm plantations. Animal-synthesized FAs showed no clear trend (Fig. 2).

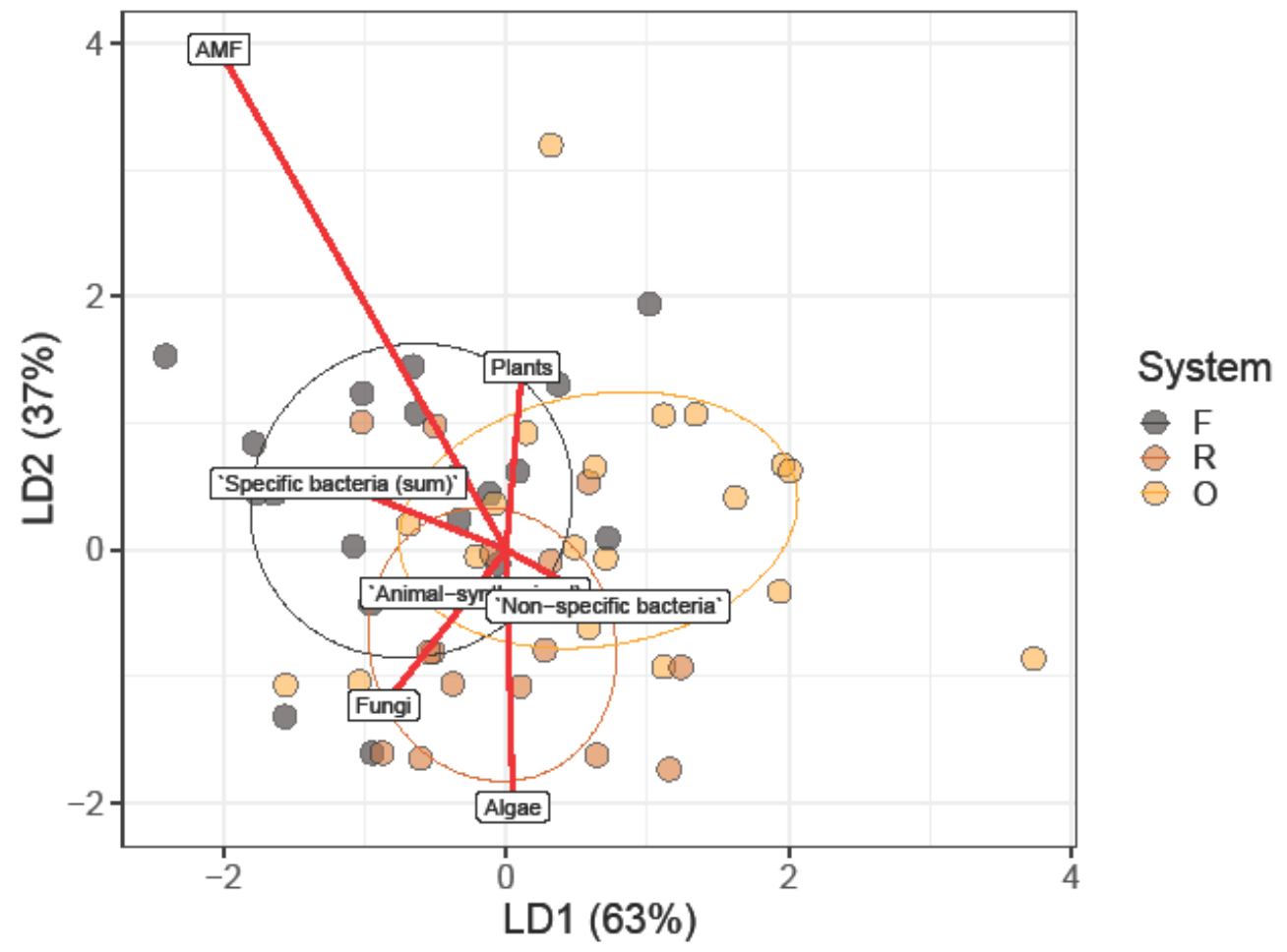

Figure 2.2. Linear discriminant analysis of biomarker FA composition in soil fauna from rainforest, rubber and oil palm plantations. Each point represents a sample (an animal or a group of animals); all groups are plotted together, seven biomarker FA indices were included in the analysis (Table 1). Colors represent land-use systems: $\mathrm{F}$ - rainforest (grey), $\mathrm{R}$ - rubber plantation (brown), $\mathrm{O}-$ oil palm plantation (orange). Ellipses are drawn on $60 \%$ confidence limits.

Overall, out of 23 biomarker FAs analyzed, seven differed significantly between land-use systems (Appendix Table 2.3). Out of eight indices analyzed, five differed significantly between land-use systems, i.e. AMF, plant, specific bacteria (Gram+ bacteria, Gram- bacteria), non-specific bacteria and plant-to-fungi ratio (Fig. 2.3). The proportion of the AMF biomarker NLFA 16:165 was ca. 33\% lower in soil fauna in rubber plantations as compared to soil fauna in rainforest. The proportion of the plant

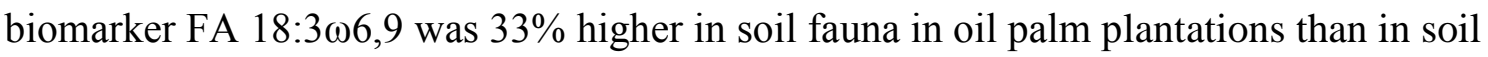


fauna in rainforest and rubber plantations. This resulted in the highest plant-to-fungi biomarker ratio in oil palm plantations. The proportion of specific bacteria biomarker FAs including those of gram+ (a15:0, i16:0, and i17:0) and gram- bacteria (cy17:0) was highest in soil fauna from rainforest and decreased gradually to rubber and oil palm plantations (in total, by about 35\%). The opposite was true for the proportion of the nonspecific bacteria biomarker FA 16:1 107 which was 70\% higher in soil fauna in oil palm plantations than in soil fauna in other land-use systems (Fig. 2.4).
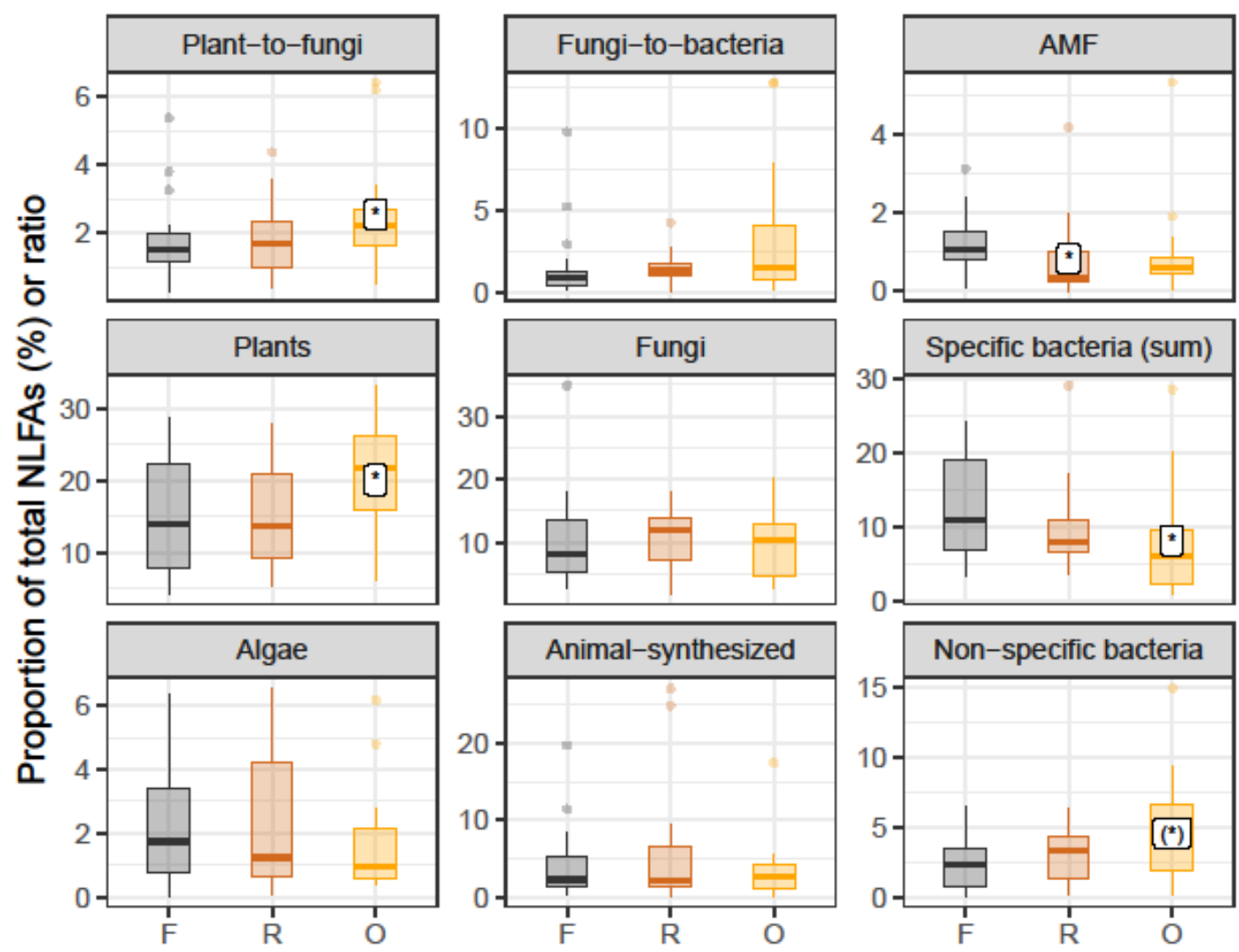

System
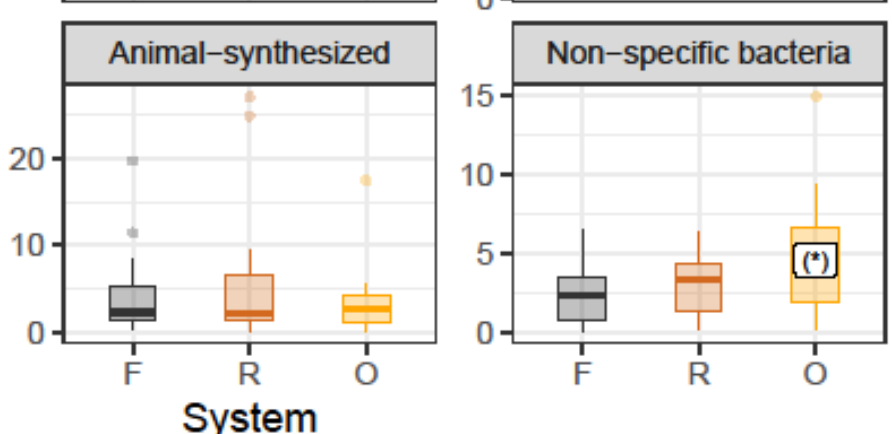

Figure 2.3. Biomarker FA-based indices in soil fauna from different land-use systems. All indices are given as proportions of total biomarker FAs except for plant-to-fungi and bacteria-to-fungi FA ratios (see methods). Data are bulked for soil fauna groups. Significant differences between plantation systems and forest (as the reference) are based on linear mixed effects models and indicated by asterisks ( $\mathrm{p}$ 0.05). Colors represent land-use systems: F - rainforest (grey), R rubber plantation (brown), $\mathrm{O}$ - oil palm plantation (orange). 


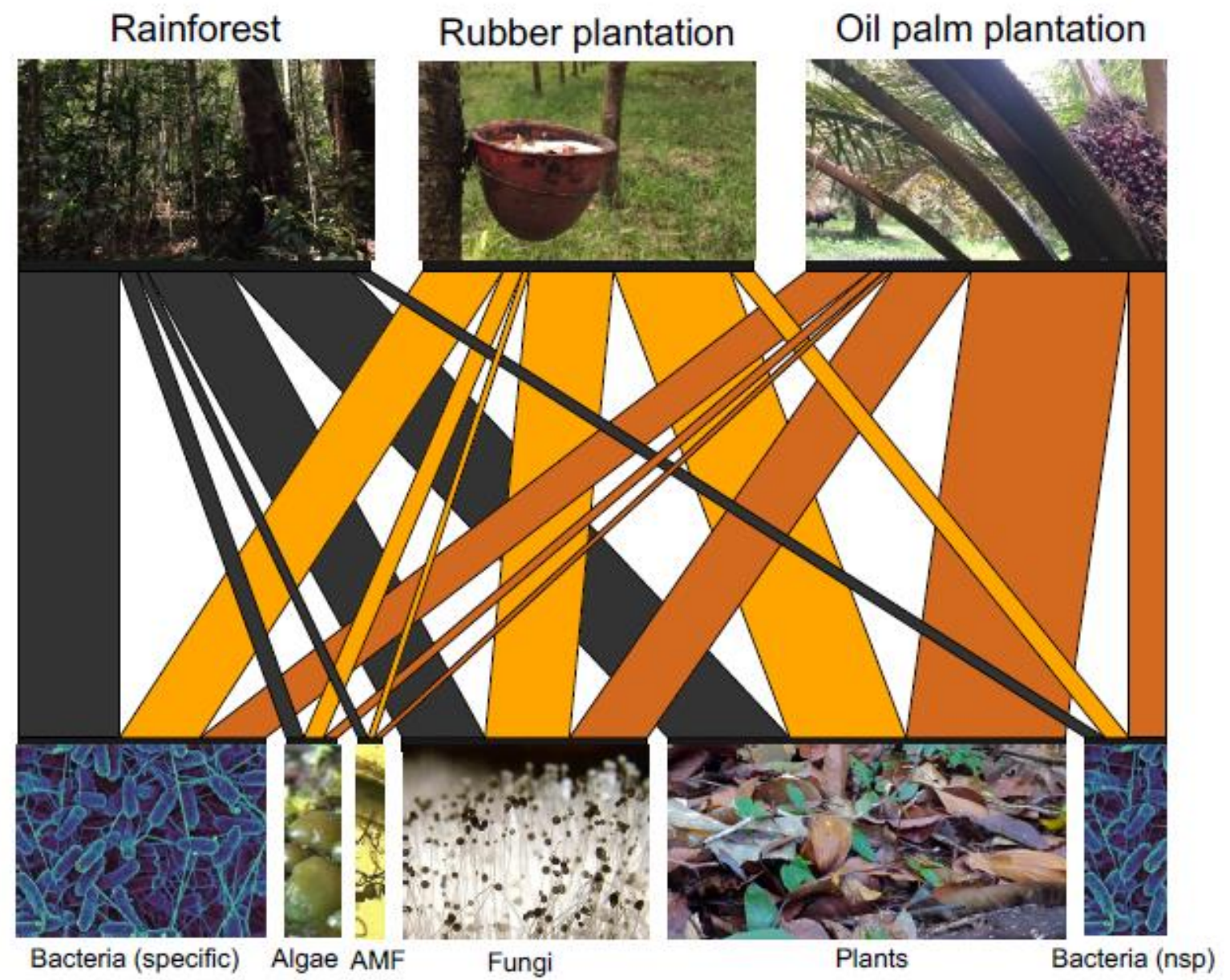

Figure 2.4. Channeling of energy from basal resources into consumers in rainforest, rubber and oil palm plantations as indicated by fatty acid analysis. The width of channels to respective land use systems reflects the relative importance of the channels as indicated by the mean proportion of biomarker NLFAs of total NLFAs in soil fauna in the respective land-use systems (data bulked across animal groups). The width of boxes / pictures of basal resources reflects the relative importance of these resources across land-use systems; note that it ignores potential differences in production and assimilation rate of different NLFAs among producer and consumer groups. Colors represent land-use systems: F - rainforest (grey), $\mathrm{R}$ - rubber plantation (brown), $\mathrm{O}$ - oil palm plantation (orange). Figure prepared using the R package bipartite.

\section{Discussion}

Conversion of rainforests into rubber and oil palm plantations alters energy channels in the soil food web as shown by different composition of biomarker FAs in soil fauna communities in different land-use systems. Our results show a general increase in the plant energy channel and strong alterations in the bacterial energy channel in oil palm plantations, and a reduction of the AMF-root-based energy channel in rubber plantations 
as compared to rainforest. Despite the general shifts in the use of basal food resources, our results also showed that different trophic groups and size classes of soil fauna contain different specific biomarker FAs across land-use systems and thus are linked to different basal resources. In most of the cases, the group identity had a larger effect on the FAs composition than land-use change and thus this effect will be discussed in the first place.

\section{Food resources of different groups of soil fauna}

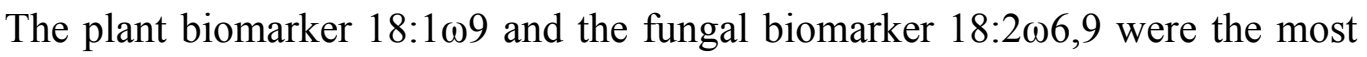
common biomarker FAs in the majority of soil fauna groups, which is in line with other studies (Ruess et al., 2002; Pollierer et al., 2012). Soils contain high amounts of plant residues which are colonized by fungi and serve as basal resources/food for many groups of detritivore soil fauna. Via predator-prey interactions, these resources are transferred to higher trophic levels (Pollierer et al., 2010; Ruess and Chamberlain, 2010). Linoleic acid $(18: 2 \omega 6,9)$ in NLFAs of soil fauna may also be derived from de novo synthesis with oleic acid 18:1 19 as precursor via lipid metabolism (Ruess et al., 2007; Menzel et al., 2017).

Contrasting our first hypothesis, the proportion of the fungal biomarker FA was unexpectedly low in Collembola and Oribatida as compared to other soil fauna groups, although these detritivore mesofauna taxa are assumed to predominantly feed on fungi (Visser et al., 1981; Klironomos and Kendrick, 1995; Scheu and Falca, 2000; Chahartaghi et al., 2005; Lenoir et al., 2007). Previous studies from temperate forest ecosystems found indeed a high proportion of fungal biomarker FAs in these groups (Haubert et al., 2004; Ruess et al., 2004; Chamberlain et al., 2005; Chen et al., 2017). Based on FA analysis, Ferlian et al. (2015) reported plant, fungi and bacteria as major resources for Collembola in a temperate ecosystem. Plant and fungal biomarker FAs represented $34 \%$ and $27 \%$ of total NLFAs in Collembola, respectively (Haubert et al., 2004) and up to $80 \%$ of total NLFAs in Oribatida (Pollierer et al., 2012). However, Ngosong et al. (2009) reported that fungi were of minor importance for Collembola nutrition in an arable soil. In our study, plant and fungal biomarker FAs represented only $13 \%$ and $4 \%$ of total NLFAs in Collembola, respectively, and $15 \%$ and $9 \%$ in Oribatida, respectively. Rather than on fungi, NLFA analysis suggests that both Collembola and Oribatida predominantly feed on bacteria (about $12-15 \%$ of their total NLFAs were represented by specific and nonspecific bacterial biomarker FAs). Further, our results indicate that in the studied tropical ecosystems both Collembola and Oribatida to some extent feed on algae, with on average 
algal biomarker FAs comprising 6\% of total NLFAs in Collembola and $4 \%$ in Oribatida. Photoautotrophic algae and bacteria may represent a considerable portion of the diet of Collembola in various habitats (Schmidt et al., 2016; Potapov et al., 2018a). A high palatability of algae for Collembola and Oribatida was demonstrated in laboratory experiments (Scheu and Folger, 2004; Buse et al., 2013, 2014; Brückner et al., 2018) and field stable isotope-based studies from temperate forest ecosystems suggested that algae serve as food resource for certain species of Collembola and Oribatida (Schneider et al., 2004; Maraun et al., 2011; Potapov et al., 2016). However, field data on algal biomarker FAs from temperate ecosystems are scarce since biomarkers for algae only were suggested recently (Buse et al., 2013, 2014). Overall, the results indicate that the diet of Collembola and Oribatida in tropical ecosystems predominantly consists of bacteria and to some extent on algae, contrasting temperate and boreal ecosystems where these animals predominantly feed on fungi and dead organic matter. This shift in diet may be related to low food quality of litter in tropical ecosystems (Illig et al., 2005; Hättenschwiler et al., 2011; Marian et al., 2017, 2018; Krashevska et al., 2018). Further, low quality of litter associated with low availability of fungi as food might be responsible for the lack of primary decomposers (Illig et al., 2005) and low abundance of Collembola and Oribatida in tropical ecosystems (Marian et al., 2018).

Biomarker FA composition of Diplopoda suggests that they predominantly feed on plant material and fungi, less on bacteria and only little on algae. This is in line with previous studies reporting that Diplopoda typically function as primary decomposers by feeding on plant litter (Bonkowski et al., 1998; Cárcamo et al., 2000). Supporting this notion, Diplopoda readily feed on decomposing leaf litter of tropical trees in the laboratory (Ashwini and Sridhar, 2005). High ${ }^{13} \mathrm{C}$ and low ${ }^{15} \mathrm{~N}$ enrichment indicates that litter-dwelling Diplopoda likely assimilate plant material colonized by saprotrophic fungi (Semenyuk et al., 2011; Potapov et al., 2013b; Potapov et al., 2018b). Similarly high concentrations of plant biomarkers in Diplopoda of temperate systems (Rawlins et al., 2006; Ambarish and Sridhar, 2015) as in the present study suggest that litter-dwelling Diplopoda occupy similar trophic niches in temperate and tropical ecosystems.

Among the soil fauna groups studied, Lumbricina contained the highest amount (on average 22\%) of C20 carbon polyunsaturated FAs (C20 PUFAs; 20:4 $66,9,12,15$ and $20: 5 \omega 3,6,9,12,15)$ which are assumed to be of animal origin (Albro, Schroeder and Corbett, 1992; Sampedro et al., 2006). As Lumbricina are detritivores that live in and feed on soil organic matter these FAs presumably are synthesized predominantly by the 
animals themselves as suggested earlier (Petersen and Holmstrup 2000, Chamberlain et $a l ., 2005)$ rather than originate from the diet. However, Sampedro et al. (2006) also found high concentrations of C20 PUFAs in the digestive tract of Lumbricina suggesting that at least in part they may also originate from the diet, possibly by feeding on protozoa and other soil microfauna (Bonkowski and Schaefer, 1997; Pokarzhevskii, 1997; Aira et al., 2003; Domínguez et al., 2003; Sampedro et al., 2006; Monroy et al., 2008). Bacterial and fungal biomarker FAs were found to comprise up to $14 \%$, whereas plant biomarker FAs only $6 \%$ of total NLFAs in Lumbricina. This also is in line with results of Sampedro et al. (2006) who showed that bacterial and fungal biomarker FAs are more abundant than plant biomarker FAs in Lumbricina suggesting that they little assimilate plant-based resources. Studies on tropical Lumbricina (Lavelle et al., 1987; Lattaud et al., 1997) also revealed that digestion system in association with soil microflora is very efficient and to digest soil organic matter through a mutualist Lumbricina microflora-digestion system and the intestinal mucus was supposed to play a central role in the process of digestion. The similarity in lipid profiles and gut content of Lumbricina in our study and published data from temperate and tropical ecosystems suggests that Lumbricina occupy a similar trophic niche across biomes and function as trophic level omnivores feeding on detritus, microorganisms and microfauna.

Araneae and Chilopoda are generalist predators which commonly feed on Collembola (Rusek, 1998; Voigtländer, 2011; Eitzinger et al., 2018). Unexpectedly and contrasting our first hypothesis, the biomarker FA composition of both Araneae and Chilopoda was different from that of Collembola, and comprised high proportions of plant and fungal biomarker FAs. Since Araneae are generalist predators, they can consume prey from both the decomposer and herbivore system (Wise, 2004, 2006; Oelbermann et al., 2008). The similarly high concentrations of plant biomarker FAs in Araneae and Chilopoda suggest that, in contrast to boreal and temperate systems, these predators heavily rely on herbivorous prey in tropical systems. Conform to this finding, generalist predators have been shown to be able to regulate populations of pest species in tropical agricultural systems (Panizzi et al., 1997; Sigsgaard, 2000). In addition, predators contained higher amounts of C20 PUFAs than decomposer animals, suggesting that in addition to synthesizing these FAs they also incorporate significant amounts from their diet which makes C20 PUFAs a potential trophic level biomarker (but there are some remarkable exceptions, such as Lumbricina). 


\section{Effect of land-use change on food resources of soil fauna}

Despite the majority of NLFAs in soil fauna in all studied land-use systems originated from plants and fungi, the relative proportions of several NLFA biomarkers varied systematically among the systems. Due to the large spatial scale of the study and the limited number of replicates analyzed variability in NLFA data was high. However, the fact that differences between land-use systems were significant suggests that changes would even be more pronounced if more data would have been available. The high abundance of plant biomarker FAs and high plant-to-fungi NLFA ratio suggests that the soil fauna is closely linked to plant basal resources in oil palm plantations. Supporting this conclusion, based on changes in stable isotope ratios, Klarner et al. (2017) found generalist litter-dwelling predators (Chilopoda) in oil palm plantations to more heavily rely on plant-based food chains than in rainforest. Our results suggest that this not only applies to generalist predators, but also to the entire soil animal community of oil palm plantations. Presumably, this is due to the well-developed herb layer in oil palm plantations and occasional weeding increasing the input of high-quality plant material to the belowground system. Overall, the results indicate that conversion of rainforest to oil palm plantations increases the plant-based energy channel in soil food webs.

The AMF biomarker NLFA showed different trends in plant litter (Krashevska et al. 2015) and in soil fauna. Investigating the same study sites, Krashevska et al. (2015) found the AMF biomarker NLFA in litter to be lowest in rainforest litter and not to differ significantly between land-use systems in soil, whereas we found the proportion of AMF biomarker NLFAs in soil fauna to be highest in rainforest. The Acrisol soils at our study sites are poor in nutrients (Allen et al., 2016), potentially forcing AMF to exploit nutrients in litter. The AMF biomarker NLFA was low in fauna from rubber plantations indicating that soil fauna little feed on AMF in this land-use system. Low AMF abundance in rubber plantations likely is related to high root phosphorus concentration (Bolan, 1991; Li et al., 2006) and reduced energy flow from leaves to roots due to cutting the phloem for collecting rubber. Our results suggest that the root-based energy channel in soil food webs of rubber plantations is reduced (Fig. 4).

In line with the study of Krashevska et al. (2015), we found the abundance of nonspecific bacterial biomarker FAs to be highest in oil palm plantations while the abundance of specific bacterial biomarker FAs decreased. Based on metagenomics, Schneider et al. (2015) also found certain bacteria taxa to be more abundant in oil palm plantations as 
compared to rainforest. The increase likely is due to more beneficial environmental factors, in particular increased soil pH (Rousk et al., 2010; Nacke et al., 2011), base saturation (Schneider et al., 2015) and/or fertilization (Shen et al., 2012). The increase in soil $\mathrm{pH}$ in plantation systems as compared to rainforest presumably is due to ashes from plant biomass burning (van Straaten et al., 2015) and liming (Krashevska et al., 2015; Allen et al., 2016). The more open canopy and improved light conditions in oil palm plantations as compared to rainforest may also favour photosynthetically active bacteria (Schneider et al., 2015). However, changes in non-specific bacterial biomarker FAs may not be related to changes in bacterial community composition in a straightforward way. De novo synthesis of non-specific bacterial biomarkers (vaccenic type FAs 16:1 107 and 18:1 17 ) was shown for fungi and protozoa (Devillard et al., 2006; Maia et al., 2007; OrRashid et al., 2007; Or-Rashid et al., 2008). Even some animals such as Collembola can synthesize these FAs (Pollierer et al., 2010). Olsson (1999) also reported that AMF can synthesize vaccenic type FA 16:1 107 . Although soil $\mathrm{pH}$ and base saturation is higher in rubber and oil palm plantations, rainforest has higher soil water content, soil $\mathrm{C}$ and $\mathrm{N}$ concentrations and amount of litter (Krashevska et al., 2015; van Straaten et al., 2015), resulting in more favourable conditions for bacteria and thereby strengthening the bacterial energy channel (Wardle et al., 2004; Fig. 4). Conform to these findings, the absolute biomarker FAs for Gram+ (a15:0, i16:0, i17:0) and Gram- bacteria (cy17:0) in soil fauna significantly declined from rainforest to rubber to oil palm plantations (Appendix Table A4). This decline mirrors the decrease in biomarker PLFAs cy17:0 and i17:0 in litter of rubber and oil palm plantations as compared to rainforest (Krashevska et al., 2015), suggesting that soil meso- and macrofauna non-selectively feed on bacteria.

\section{Conclusions}

The results suggest that different high-rank taxonomic groups are linked to different resources. Fungi appear to contribute only little to the nutrition of detritivore mesofauna taxa, in particular Collembola, contrasting temperate and boreal ecosystems. Further, algae are likely to play a more important role as food resource of mesofauna (Collembola and Oribatida) in tropical than temperate ecosystems. Litter-dwelling macrofauna (Araneae, Chilopoda and Diplopoda) heavily rely on fungal- and plant-based resources, while soil-dwelling Lumbricina mostly rely on microbial resources, but only little on plant-based resources such as leaf litter. Differences between soil fauna groups 
were rather constant across land-use systems, suggesting a consistent niche differentiation between different trophic groups and body size classes (meso- and macrofauna) in soil. The unexpected differences in trophic niches of some fauna groups between temperate and tropical ecosystems call for further studies to understand the driving factors for these differences.

Changes in energy channels with conversion of rainforest into plantation systems suggest that soil fauna communities respond in a flexible way to changes in the availability of food resources. Soil fauna in oil palm plantations predominantly rely on plant-based resources. By contrast, the soil fauna community in rainforest heavily relies on the bacterial energy channel. The contribution of AMF in fuelling soil food webs appears to be particularly low in rubber plantations, presumably due to alleviated limitation by phosphorus and by cutting the phloem for harvesting rubber. These alterations suggest that land-use change alters food-web structure and may disrupt interaction networks resulting in changes in ecosystem functioning and food-web resilience that are hard to predict with the current limited knowledge on tropical belowground systems.

\section{Acknowledgements}

Financial support was provided by the German Research Foundation (DFG) in the framework of the collaborative German-Indonesian research project CRC990 (EFForTS). We thank the State Ministry of Research and Technology of Indonesia (RISTEK), the Indonesian Institute of Sciences (LIPI), Ministry of Forestry (PHKA) and Restoration Ecosystem Indonesia Harapan for the permits. We thank Indonesian organizations and farmers for granting access to the sampling plots and use of their properties. We acknowledge support by the German Research Foundation and the Open Access Publication Funds of the University of Göttingen. 
Chapter 2

\section{Appendix}

Table 2.1. Average proportions of biomarker FAs in dominating taxa of soil animals.

\begin{tabular}{|c|c|c|c|c|c|c|}
\hline Biomarker, NLFA & Araneae & Chilopoda & Collembola & Diplopoda & Lumbricina & Oribatida \\
\hline \multicolumn{7}{|l|}{ Algae } \\
\hline $16: 2 \omega 6,9$ & $0.23 \pm 0.14$ & $0.2 \pm 0.14$ & $0.19 \pm 0.27$ & $0.22 \pm 0.24(5)$ & $0.14 \pm 0.12$ & $0.43 \pm 0.31$ \\
\hline $16: 3 \omega 3,6,9$ & $0.65 \pm 0.62(5) \mathrm{c}$ & $0.78 \pm 0.2(4) \mathrm{c}$ & $5.46 \pm 1.21(5) \mathrm{a}$ & $1.8 \pm 1.43(5) \mathrm{bc}$ & $0.84 \pm 0.74(4) \mathrm{c}$ & $3.78 \pm 1.37(5) \mathrm{ab}$ \\
\hline \multicolumn{7}{|l|}{ AMF } \\
\hline $16: 1 \omega 5$ & $0.89 \pm 0.72(5)$ & $0.51 \pm 0.11$ & $1.51 \pm 1.66(5)$ & $0.99 \pm 0.91(5)$ & $1.1 \pm 0.24$ & $0.91 \pm 0.74(5)$ \\
\hline \multicolumn{7}{|l|}{ Animal-synthesized } \\
\hline $20: 4 \omega 6$ & $4.08 \pm 1.51(5) \mathrm{b}$ & $4.24 \pm 2.03(4) \mathrm{b}$ & $1.22 \pm 0.86(5) \mathrm{c}$ & $1.24 \pm 0.47(5) \mathrm{c}$ & $18.13 \pm 3.29(4) \mathrm{a}$ & $0.42 \pm 0.58(5) \mathrm{c}$ \\
\hline $20: 5 \omega 3$ & $1.52 \pm 0.85(5) b$ & $1.22 \pm 0.76(4) \mathrm{bc}$ & $0.5 \pm 0.58(5) \mathrm{bc}$ & $0.17 \pm 0.25(5) \mathrm{c}$ & $4.15 \pm 1.21(4) \mathrm{a}$ & $0.29 \pm 0.47(5) \mathrm{c}$ \\
\hline \multicolumn{7}{|l|}{ Fungi } \\
\hline $18: 2 \omega 6,9$ & $15.64 \pm 4.18(5) \mathrm{a}$ & $11.51 \pm 2.76(4) \mathrm{a}$ & $4.31 \pm 2.06(5) \mathrm{b}$ & $11.6 \pm 3.89(5) \mathrm{a}$ & $13.8 \pm 1.13(4) \mathrm{a}$ & $9.03 \pm 6.37(5) \mathrm{ab}$ \\
\hline \multicolumn{7}{|c|}{ Non-specific bacteria } \\
\hline $18: 1 \omega 7$ & $1.48 \pm 0.62(5) \mathrm{ab}$ & $1.13 \pm 0.34(4) \mathrm{ab}$ & $0.36 \pm 0.29(5) \mathrm{bc}$ & $2.41 \pm 1.83(5) \mathrm{a}$ & $3.12 \pm 2.06(4) \mathrm{a}$ & $0.12 \pm 0.19(5) \mathrm{c}$ \\
\hline \multicolumn{7}{|c|}{ Gram-negative bacteria } \\
\hline 2-OH 12:0 & $0.16 \pm 0.3(5)$ & $0.38 \pm 0.33(4)$ & $0.37 \pm 0.45(5)$ & $0.18 \pm 0.35(5)$ & $0.33 \pm 0.4(4)$ & $0.8 \pm 1.63$ \\
\hline 3-OH 12:0 & $0.23 \pm 0.21$ & $0.56 \pm 0.42$ & $0.58 \pm 0.65(5)$ & $0.47 \pm 0.47(5)$ & $0.32 \pm 0.37$ & $1 \pm 1.44(5)$ \\
\hline 2-OH 14:0 & $0.05 \pm 0.03(5) \mathrm{ab}$ & $0.02 \pm 0.01(4) b$ & $0.07 \pm 0.05(5) \mathrm{ab}$ & $0.03 \pm 0.02(5) b$ & $0.02 \pm 0.01(4) b$ & $0.09 \pm 0.05(5) \mathrm{a}$ \\
\hline $3-\mathrm{OH}$ 14:0 & $0.12 \pm 0.1$ & $0.04 \pm 0.06$ & $0.1 \pm 0.16$ & $0.04 \pm 0.02$ & $0.13 \pm 0.08$ & $0.1 \pm 0.1(5)$ \\
\hline
\end{tabular}


Chapter 2

\begin{tabular}{|c|c|c|c|c|c|c|}
\hline cy17:0 & $0.14 \pm 0.15(5) a b$ & $0.4 \pm 0.14(4) \mathrm{ab}$ & $1.47 \pm 1.7(5) \mathrm{a}$ & $1.22 \pm 1.91(5) \mathrm{ab}$ & $0.3 \pm 0.28(4) \mathrm{ab}$ & $0.07 \pm 0.14(5) b$ \\
\hline 2-OH 16:0 & $0.13 \pm 0.13(5)$ & $0.09 \pm 0.09$ & $0.22 \pm 0.27(5)$ & $0.13 \pm 0.21$ & $0.42 \pm 0.14$ & $0.27 \pm 0.24(5)$ \\
\hline \multicolumn{7}{|l|}{ cy19:0 } \\
\hline \multicolumn{7}{|c|}{ Gram-positive bacteria } \\
\hline $\mathrm{i} 15: 0$ & $1.98 \pm 1.57(5)$ & $1.58 \pm 0.16(4)$ & $3.89 \pm 1.24(5)$ & $3.31 \pm 3.26(5)$ & $2.87 \pm 0.62$ & $2.3 \pm 2.14(5)$ \\
\hline a15:0 & $1.94 \pm 1.47(5)$ & $1.4 \pm 0.5(4)$ & $4.68 \pm 3.01(5)$ & $2.02 \pm 2.21(5)$ & $1.8 \pm 0.64$ & $4.52 \pm 2.77(5)$ \\
\hline i16:0 & $0.72 \pm 0.58(5) b$ & $0.5 \pm 0.3(4) b$ & $0.43 \pm 0.16(5) b$ & $0.51 \pm 0.33(5) \mathrm{b}$ & $1.69 \pm 0.17(4) \mathrm{a}$ & $0.29 \pm 0.38(5) b$ \\
\hline i17:0 & $0.88 \pm 1.15(5)$ & $0.44 \pm 0.25$ & $0.97 \pm 0.84(5)$ & $0.69 \pm 0.5(5)$ & $0.84 \pm 0.62$ & $0.54 \pm 0.44(5)$ \\
\hline \multicolumn{7}{|l|}{ Plants } \\
\hline $18: 3 \omega 6,9,12$ & $0.13 \pm 0.11(5)$ & $0.26 \pm 0.11$ & $0.03 \pm 0.04$ & $2.66 \pm 5.59(5)$ & $0.14 \pm 0.06$ & $0.08 \pm 0.16(5)$ \\
\hline $18: 1 \omega 9$ & $20.49 \pm 5.46(5) \mathrm{ab}$ & $24.92 \pm 1.16(4) \mathrm{a}$ & $11.68 \pm 5.21(5) \mathrm{bc}$ & $18.51 \pm 9.66(5) \mathrm{ab}$ & $5.81 \pm 1.19(4) \mathrm{c}$ & $12.82 \pm 8.65(5) \mathrm{ac}$ \\
\hline $22: 0$ & $0.34 \pm 0.52(5)$ & $0.17 \pm 0.17$ & $0.58 \pm 0.47(5)$ & $0.4 \pm 0.46(5)$ & $0.28 \pm 0.38$ & $0.16 \pm 0.23(5)$ \\
\hline $24: 0$ & $0.04 \pm 0.07$ & $0 \pm 0(4)$ & $0.08 \pm 0.07(5)$ & $0.44 \pm 0.84(5)$ & $0.03 \pm 0.03$ & $2.24 \pm 4.84(5)$ \\
\hline
\end{tabular}

*Animal-synthesized C20 polyunsaturated FAs cannot be used as biomarkers for animal consumption 
Table 2.2. LDA coefficients for each of the analyzed biomarker FA indices.

\begin{tabular}{lcc}
\hline Biomarker NLFA & LD1 & LD2 \\
\hline Algae & 0.24 & -10.14 \\
AMF & -10.14 & 19.78 \\
Animal-synthesized* & -1.10 & -1.69 \\
Non-spesific bacteria & 3.62 & -2.24 \\
Spesific bacteria (sum) & -5.92 & 2.62 \\
Fungi & -4.33 & -6.18 \\
Plants & 0.56 & 7.18
\end{tabular}

*Animal-synthesized C20 polyunsaturated FAs cannot be used as biomarkers for animal consumption 
Table 2.3. Biomarker FAs composition in soil animals from different land-use systems. Means and standard deviations across all taxa are shown (number of replicates is given in brackets). Significant differences from forest based on linear mixed-effects models are shown in bold; *p $<0.05 ; * * \mathrm{p}<0.01 ; * * * \mathrm{p}<0.001$.

\begin{tabular}{|c|c|c|c|c|}
\hline Biomarker & FA & Rainforest & Rubber plantation & Oil palm plantation \\
\hline AMF & $16: 1 \omega 5$ & $1.24 \pm 0.72(19)$ & $0.81 \pm 1.12(15)^{*}$ & $0.93 \pm 1.23(17)$ \\
\hline Algae & $16: 2 \omega 6,9$ & $0.27 \pm 0.32(19)$ & $0.16 \pm 0.1(15)$ & $0.34 \pm 0.3(17)$ \\
\hline Algae & $16: 3 \omega 3,6,9$ & $1.95 \pm 1.63(19)$ & $2.34 \pm 2.34(15)$ & $1.39 \pm 1.63(17)$ \\
\hline Animal-synthesized ${ }^{*}$ & $20: 4 \omega 6,9,12,15$ & $3.38 \pm 3.85(19)$ & $4.75 \pm 6.91(15)$ & $2.58 \pm 3.38(17)$ \\
\hline Animal-synthesized ${ }^{*}$ & $20: 5 \omega 3,6,9,12,15$ & $0.84 \pm 0.98$ & $1.18 \pm 1.77(15)$ & $0.69 \pm 0.83(17)$ \\
\hline Fungi & $18: 2 \omega 6,9$ & $10.42 \pm 7.33(19)$ & $10.65 \pm 5.06(15)$ & $9.82 \pm 5.27(17)$ \\
\hline General bacteria & $16: 1 \omega 7$ & $0.91 \pm 1.44(19)$ & $1.59 \pm 2.17(15)$ & $3.23 \pm 2.49(17)^{* * * *}$ \\
\hline General bacteria & $18: 1 \omega 7$ & $1.53 \pm 1.37(19)$ & $1.46 \pm 1.43(15)$ & $1.35 \pm 2.12(17)$ \\
\hline $\mathrm{Gram}^{+}$-bacteria & i15:0 & $2.58 \pm 1.54(19)$ & $3.57 \pm 2.64(15)$ & $2.5 \pm 2.22(17)$ \\
\hline $\mathrm{Gram}^{+}$-bacteria & a15:0 & $4.18 \pm 3.07(19)$ & $2.24 \pm 1.64(15)^{*}$ & $2.2 \pm 1.83(17)^{*}$ \\
\hline $\mathrm{Gram}^{+}$-bacteria & i16:0 & $0.86 \pm 0.73(19)$ & $0.69 \pm 0.48(15)$ & $0.34 \pm 0.43(17)^{* *}$ \\
\hline $\mathrm{Gram}^{+}$-bacteria & i17:0 & $1.59 \pm 1.58(19)$ & $0.71 \pm 0.83(15)^{*}$ & $0.56 \pm 0.77(17)^{* *}$ \\
\hline Gram ${ }^{-}$bacteria & 2-OH 12:0 & $0.45 \pm 0.92(19)$ & $0.61 \pm 1.03(15)$ & $0.45 \pm 0.84(17)$ \\
\hline Gram ${ }^{-}$bacteria & 3-OH 12:0 & $0.53 \pm 0.84(19)$ & $0.87 \pm 1.68(15)$ & $0.57 \pm 1.09(17)$ \\
\hline Gram ${ }^{-}$bacteria & 2-OH 14:0 & $0.04 \pm 0.04$ (19) & $0.05 \pm 0.03(15)$ & $0.05 \pm 0.07(17)$ \\
\hline Gram ${ }^{-}$bacteria & $3-\mathrm{OH} 14: 0$ & $0.08 \pm 0.15(19)$ & $0.11 \pm 0.1(15)$ & $0.03 \pm 0.06(17)$ \\
\hline Gram ${ }^{-}$bacteria & cy17:0 & $1.37 \pm 1.63(19)$ & $0.5 \pm 1.09(15)^{*}$ & $0.55 \pm 0.82(17)^{*}$ \\
\hline Gram ${ }^{-}$bacteria & 2-OH 16:0 & $0.21 \pm 0.21(19)$ & $0.27 \pm 0.24(15)$ & $0.13 \pm 0.16(17)$ \\
\hline Gram ${ }^{-}$bacteria & су19:0 & $0.75 \pm 0.86(19)$ & $0.54 \pm 0.77(15)$ & $0.87 \pm 1.01(17)$ \\
\hline Plants & $18: 3 \omega 6,9,12$ & $0.13 \pm 0.13(19)$ & $1 \pm 3.23(15)$ & $0.09 \pm 0.07(17)$ \\
\hline Plants & $18: 1 \omega 9$ & $15.39 \pm 8.46(19)$ & $14.69 \pm 7.76(15)$ & $20.05 \pm 7.99(17)^{*}$ \\
\hline Plants & $22: 0$ & $0.43 \pm 0.41(19)$ & $0.17 \pm 0.24(15)$ & $0.23 \pm 0.39(17)$ \\
\hline Plants & $24: 0$ & $0.18 \pm 0.43(19)$ & $0.74 \pm 2.81(15)$ & $0.03 \pm 0.05(17)$ \\
\hline
\end{tabular}


Supplementary Table S2.1. List of soil fauna used for NLFA analyzes.

\begin{tabular}{|c|c|c|c|c|c|c|}
\hline No. & System & Plot_ID & Group & Subroup & Number of animals & Comments \\
\hline 1 & Rainforest & HF1\&3 & Araneae & Araneae & 8 & HF1 and HF3, small free-hunting Araneae \\
\hline 2 & Rainforest & HF2 & Blattodea & Blattodea & 1 & Large size, wingless \\
\hline 3 & Rainforest & HF1\&3 & Diplura & Campodeidae & 5 & $\mathrm{HF} 2$ and HF3 \\
\hline 4 & Rainforest & $\mathrm{HF} 2$ & Coleoptera & Carabidae - imago & 6 & Small size, brown \\
\hline 5 & Rainforest & HF4 & Coleoptera & Carabidae - imago & 8 & Small size, brown \\
\hline 6 & Rainforest & HF1\&3 & Lumbricina & Clitellata & 1 & \\
\hline 7 & Rainforest & $\mathrm{HF} 1 \& 3$ & Lumbricina & Clitellata & 2 & Medium size \\
\hline 8 & Rainforest & HF1\&3 & Lumbricina & Clitellata & & \\
\hline 9 & Rainforest & $\mathrm{HF} 1 \& 3$ & Coleoptera & Coleoptera - imago & 1 & cf. Agathidium \\
\hline 10 & Rainforest & HF1\&3 & Coleoptera & Coleoptera - larvae & 2 & HF1 and HF3 larvae cf. Elateridae \\
\hline 11 & Rainforest & HF1\&3 & Coleoptera & Coleoptera - larvae & 1 & larvae cf. Elateridae, 1 large \\
\hline 12 & Rainforest & $\mathrm{HF} 4$ & Coleoptera & Coleoptera - larvae & 3 & larvae cf. Elateridae, 1 large, 2 small \\
\hline 13 & Rainforest & HF2 & Chilopoda & Geophilomorpha & 1 & Large size \\
\hline 14 & Rainforest & $\mathrm{HF} 2$ & Chilopoda & Geophilomorpha & 1 & Medium size \\
\hline 15 & Rainforest & $\mathrm{HF} 2$ & Chilopoda & Geophilomorpha & 1 & Medium size \\
\hline 16 & Rainforest & HF4 & Araneae & Hunting Araneae & 4 & Pale, medium size, free-hunting \\
\hline 17 & Rainforest & HF4 & Isopoda & Isopoda & 1 & juvenile cf. Oniscidae \\
\hline 18 & Rainforest & HF1 \&3 & Diplura & Japygidae & 25 & HF1 and HF3 \\
\hline 19 & Rainforest & $\mathrm{HF} 2$ & Collembola & Lepidocyrtini & 13 & Sinella + Lepidocyrtus \\
\hline 20 & Rainforest & HF4 & Collembola & Lepidocyrtini & 50 & Sinella + Lepidocyrtus \\
\hline 21 & Rainforest & HF2 & Mesostigmata & Mesostigmata & 15 & Gamasina \\
\hline 22 & Rainforest & HF4 & Opiliones & Opiliones & 2 & Small size, „mite-harvestman“ \\
\hline 23 & Rainforest & HF2 & Oribatida & Oribatida & 23 & Galumnidae, Otocepheidae, Galumnellidae, Scheloribatidae \\
\hline 24 & Rainforest & HF4 & Oribatida & Oribatida & 34 & Galumnidae, Haplozetidae, Galumnellidae, Zetordestidae \\
\hline 25 & Rainforest & $\mathrm{HF} 2$ & Collembola & Paronellidae & 1 & 1 large \\
\hline
\end{tabular}


Chapter 2

\begin{tabular}{|c|c|c|c|c|c|c|}
\hline 26 & Rainforest & HF1\&3 & Diplopoda & Polydesmidae & 1 & Medium size \\
\hline 27 & Rainforest & $\mathrm{HF} 2$ & Diplopoda & Polydesmidae & 3 & Medium size \\
\hline 28 & Rainforest & $\mathrm{HF} 2$ & Diplopoda & Polydesmidae & 1 & Medium size \\
\hline 29 & Rainforest & $\mathrm{HF} 2$ & Pseudoscorpionida & Pseudoscorpionida & 16 & \\
\hline 30 & Rainforest & HF4 & Schizomida & Schizomida & 2 & 1 large, 1 small \\
\hline 31 & Rainforest & HF1\&3 & Symphyla & Symphyla & 54 & $\mathrm{HF} 1$ and $\mathrm{HF} 3$ \\
\hline 32 & Rainforest & HF2 & Collembola & Symphypleona & 8 & \\
\hline 33 & Oil Palm & $\mathrm{HO} 2$ & Araneae & Araneae & 4 & $\begin{array}{l}1 \text { small }+1 \text { mrdium brown white legs, } 1 \text { brown small, } 1 \\
\text { black small white legs, free hunters }\end{array}$ \\
\hline 34 & Oil Palm & HO1 & Blattodea & Blattodea & 1 & Juvenile \\
\hline 35 & Oil Palm & $\mathrm{HO} 2$ & Blattodea & Blattodea & 1 & Juvenile \\
\hline 36 & Oil Palm & $\mathrm{HO} 3 \& 4$ & Blattodea & Blattodea & 1 & Juvenile \\
\hline 37 & Oil Palm & $\mathrm{HO} 3 \& 4$ & Lumbricina & Clitellata & 1 & \\
\hline 38 & Oil Palm & HO1 & Dermaptera & Dermaptera & 2 & Medium, brown \\
\hline 39 & Oil Palm & HO1 & Dermaptera & Dermaptera & 2 & Medium, brown \\
\hline 40 & Oil Palm & $\mathrm{HO} 2$ & Dermaptera & Dermaptera & 3 & Juvenile \\
\hline 41 & Oil Palm & HO1 & Chilopoda & Geophilomorpha & 2 & Medium size \\
\hline 42 & Oil Palm & $\mathrm{HO} 1$ & Chilopoda & Geophilomorpha & 2 & Medium size \\
\hline 43 & Oil Palm & $\mathrm{HO} 2$ & Chilopoda & Geophilomorpha & 1 & 1 small \\
\hline 44 & Oil Palm & $\mathrm{HO} 2$ & Chilopoda & Geophilomorpha & 1 & 1 medium size \\
\hline 45 & Oil Palm & HO1 & Araneae & Hunting Araneae & 4 & $\begin{array}{l}1 \text { black small, } 1 \text { black small white legs, } 1 \text { medium black, } \\
\text { free-hunters }\end{array}$ \\
\hline 46 & Oil Palm & HO1 & Araneae & Hunting Araneae & 4 & $\begin{array}{l}1 \text { small }+1 \text { mrdium brown white legs, } 1 \text { brown small, } 1 \\
\text { black small white legs, free hunters }\end{array}$ \\
\hline 47 & Oil Palm & $\mathrm{HO} 2$ & Araneae & Hunting Araneae & 1 & 1 black large \\
\hline 48 & Oil Palm & $\mathrm{HO} 2$ & Araneae & Hunting Araneae & 1 & Large size, black, free hunter \\
\hline 49 & Oil Palm & HO1 & Isopoda & Isopoda & 2 & Medium size cf. Oniscidae \\
\hline 50 & Oil Palm & HO1 & Isopoda & Isopoda & 3 & Medium size cf. Oniscidae \\
\hline 51 & Oil Palm & $\mathrm{HO} 2$ & Isopoda & Isopoda & 4 & Medium size cf. Oniscidae +3 small \\
\hline
\end{tabular}


Chapter 2

\begin{tabular}{|c|c|c|c|c|c|c|}
\hline 52 & Oil Palm & HO1 & Diplopoda & Julidae & 3 & Medium size \\
\hline 53 & Oil Palm & HO1 & Diplopoda & Julidae & 3 & Medium size \\
\hline 54 & Oil Palm & $\mathrm{HO} 3 \& 4$ & Collembola & Lepidocyrtini & 27 & \\
\hline 55 & Oil Palm & $\mathrm{HO} 2$ & Lepidoptera & Lepidoptera & 1 & medium, grey, stock \\
\hline 56 & Oil Palm & HO1 & Mesostigmata & Mesostigmata & 10 & Uropodina \\
\hline 57 & Oil Palm & $\mathrm{HO} 2$ & Mesostigmata & Mesostigmata & 34 & Uropodina \\
\hline 58 & Oil Palm & $\mathrm{HO} 2$ & Mesostigmata & Mesostigmata & 21 & Gamasina \\
\hline 59 & Oil Palm & $\mathrm{HO} 2$ & NA & Mixed sample & $5,3,2$ & Pseudoscorpion+Entomobryidae+Coleoptera \\
\hline 60 & Oil Palm & HO1 & Opiliones & Opiliones & 1 & Small size \\
\hline 61 & Oil Palm & $\mathrm{HO} 2$ & Oribatida & Oribatida & 28 & $\begin{array}{l}\text { Lohmanniididae, Otocepheidae, Galumnellidae, } \\
\text { Scheloribatidae }\end{array}$ \\
\hline 62 & Oil Palm & HO1 & Diplopoda & Polydesmidae & 2 & \\
\hline 63 & Oil Palm & $\mathrm{HO} 1$ & Diplopoda & Polydesmidae & 1 & \\
\hline 64 & Oil Palm & $\mathrm{HO} 2$ & Chilopoda & Scolopendromorpha & 3 & 2 small juvenile, 1 medium \\
\hline 65 & Oil Palm & $\mathrm{HO} 3 \& 4$ & Symphyla & Symphyla & 10 & \\
\hline 66 & Rubber & HR2 & Araneae & Araneae & 4 & \\
\hline 67 & Rubber & HR2 & Coleoptera & Carabidae - imago & 1 & Medium size, black, cf. Nebria \\
\hline 68 & Rubber & HR2 & Lumbricina & Clitellata & 1 & \\
\hline 69 & Rubber & HR2 & Lumbricina & Clitellata & 1 & \\
\hline 70 & Rubber & HR3 & Lumbricina & Clitellata & 1 & large (piece) \\
\hline 71 & Rubber & HR2 & Coleoptera & Coleoptera - predator larvae & 4 & Medium/small size, predator \\
\hline 72 & Rubber & HR2 & Collembola & $\begin{array}{l}\text { Epigeic } \\
\text { Entomobryidae/Paronellidae }\end{array}$ & 10 & Cingula \\
\hline 73 & Rubber & HR3 & Chilopoda & Geophilomorpha & 1 & large (dead) \\
\hline 74 & Rubber & HR2 & Diplura & Japygidae+Campodeidae & 4 & \\
\hline 75 & Rubber & HR1 & Collembola & Lepidocyrtini & 21 & Sinella \\
\hline
\end{tabular}


Chapter 2

\begin{tabular}{|c|c|c|c|c|c|c|}
\hline 76 & Rubber & HR2 & Collembola & Lepidocyrtini & 30 & \\
\hline 77 & Rubber & HR2 & Opiliones & Opiliones & 1 & \\
\hline 78 & Rubber & HR $1 \& 3$ & Oribatida & Oribatida & 8 & Lohmanniididae \\
\hline 79 & Rubber & HR2 & Oribatida & Oribatida & 17 & Galumnidae, Galumnellidae \\
\hline 80 & Rubber & HR2 & Diplopoda & Polydesmidae & 2 & 1 from HR4 \\
\hline 81 & Rubber & HR4 & Diplopoda & Polydesmidae & 1 & \\
\hline 82 & Rubber & HR4 & Pseudoscorpionida & Pseudoscorpionida & 22 & \\
\hline 83 & Rubber & HR2 & Psocoptera & Psocoptera & 10 & different ages \\
\hline 84 & Rubber & HR2 & Schizomida & Schizomida & 1 & \\
\hline 85 & Rubber & HR2 & Symphyla & Symphyla & 2 & \\
\hline 86 & Rubber & HR2 & Collembola & Symphypleona & 7 & \\
\hline
\end{tabular}


Chapter 2

Supplementary Table S2.2. Plot-based mean values of NLFA proportions in soil fauna groups.

\begin{tabular}{|c|c|c|c|c|c|c|c|c|c|c|c|c|c|c|c|c|c|c|c|c|c|c|c|c|c|}
\hline & & Sys & & & & & & & & & & & & & & & & & & & & 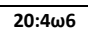 & $20: 5 \omega 3$, & & \\
\hline \multirow[b]{2}{*}{ Group } & Plo & te & 2-OH & 3-OH & & & 2-OH & 3-OH & & $16: 2$ & $16: 3 \omega$ & $16: 1 \omega$ & $16: 1 \omega$ & & & 2-OH & $18: 3 \omega$ & $18: 2 \mathrm{w}$ & 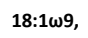 & $18: 1 \omega 7$ & & ,9,12,1 & $6,9,12,1$ & \multirow[b]{2}{*}{ 22:0 } & \multirow[b]{2}{*}{$24: 0$} \\
\hline & $t$ & $\mathrm{~m}$ & 12:0 & 12:0 & i15:0 & a15:0 & $14: 0$ & $14: 0$ & i16:0 & $\omega 6,9$ & $3,6,9$ & 7 & 5 & i17:0 & сy17:0 & $16: 0$ & 6 & 6,9 & $18: 3 \omega 3$ & & cy19:0 & 5 & 5 & & \\
\hline & $\mathrm{HF}$ & & & & & & & & & & & & & & & & & & & & & & & & \\
\hline & $1 \&$ & & & & & & & & & & & & & & & & & & & & & & & & \\
\hline \multirow[t]{2}{*}{ Araneae } & 3 & $\mathrm{~F}$ & 0 & 0.32 & 2.03 & 3.2 & 0.06 & 0.16 & 1.32 & 0.12 & 1.62 & 0.91 & 1.76 & 0.54 & 0 & 0 & 0.01 & 11.02 & 21.35 & 1.36 & 0.43 & 4.49 & 1.92 & 1.26 & 0.16 \\
\hline & $\mathrm{HF}$ & & & & & & & & & & & & & & & & & & & & & & & & \\
\hline \multirow[t]{3}{*}{ Araneae } & 4 & $\mathrm{~F}$ & 0.05 & 0.07 & 0.94 & 0.39 & 0.02 & 0.17 & 1.36 & 0.43 & 0.72 & 0.56 & 1.44 & 0.18 & 0 & 0.18 & 0.29 & 17.96 & 22.87 & 2.09 & 0.07 & 6.25 & 2.14 & 0 & 0 \\
\hline & & & $0.03 \pm$ & $0.03 \pm$ & $0.22 \pm$ & & $0.02 \pm$ & & & $0.26 \pm$ & & & $0.46 \pm$ & $0.16 \pm$ & $0.13 \pm$ & $0.11 \pm$ & $0.05 \pm$ & $17.45 \pm$ & & & $0.64 \pm$ & & & $0.22 \pm$ & \\
\hline & HO & & 0.01 & 0.04 & 0.08 & $0.3 \pm 0$. & 0.02 & $0 \pm 0.0$ & $0.1 \pm 0$ & 0.19 & $0.66 \pm$ & $8.03 \pm 9$ & 0.11 & 0.23 & 0.03 & 0.08 & 0.03 & 1.86 & $23.65 \pm 3$ & $0.72 \pm 0$ & 0.52 & $3.64 \pm 1$. & $1.9 \pm 1.39$ & 0.28 & $0 \pm 0.0$ \\
\hline \multirow[t]{3}{*}{ Araneae } & 1 & 0 & (2) & (2) & (2) & $16(2)$ & (2) & $1(2)$ & $06(2)$ & (2) & $0.8(2)$ & $.04(2)$ & (2) & (2) & (2) & (2) & (2) & (2) & $.2(2)$ & $24(2)$ & (2) & $34(2)$ & (2) & (2) & $1(2)$ \\
\hline & & & & $0.56 \pm$ & $2.35 \pm$ & $2.65 \pm$ & $0.06 \pm$ & $0.02 \pm$ & $0.39 \pm$ & & $0.26 \pm$ & & & $0.62 \pm$ & $0.35 \pm$ & $0.05 \pm$ & $0.19 \pm$ & $20.35 \pm$ & & & $1.33 \pm$ & & & $0.04 \pm$ & $0.02 \pm$ \\
\hline & Hо & & $0.7 \pm 1$. & 0.93 & 1.65 & 1.28 & 0.04 & 0.03 & 0.44 & $0.27 \pm$ & 0.22 & $2.58 \pm 2$ & $0.8 \pm 0$ & 0.65 & 0.33 & 0.06 & 0.28 & 6.29 & $23.69 \pm 7$ & $1.1 \pm 0.8$ & 1.15 & $3.97 \pm 0$ & $1.62 \pm 0.7$ & 0.04 & 0.04 \\
\hline \multirow[t]{2}{*}{ Araneae } & 2 & 0 & $2(3)$ & (3) & (3) & (3) & (3) & (3) & (3) & $0.3(3)$ & (3) & $.05(3)$ & $53(3)$ & (3) & (3) & (3) & (3) & (3) & $.81(3)$ & $9(3)$ & (3) & $86(3)$ & $3(3)$ & (3) & (3) \\
\hline & HR & & & & & & & & & & & & & & & & & & & & & & & & \\
\hline Araneae & 2 & $\mathrm{R}$ & 0 & 0.17 & 4.34 & 3.15 & 0.1 & 0.22 & 0.41 & 0.06 & 0 & 0.11 & 0 & 2.91 & 0.22 & 0.34 & 0.09 & 11.42 & 10.87 & 2.13 & 2.51 & 2.07 & 0.03 & 0.18 & 0 \\
\hline Blattode & $\mathrm{HF}$ & & & & & & & & & & & & & & & & & & & & & & & & \\
\hline a & 2 & $\mathrm{~F}$ & 0 & 0.01 & 0.91 & 0.2 & 0.01 & 0.02 & 0.74 & 0.09 & 0.32 & 1.16 & 1.42 & 0.83 & 0.69 & 0.03 & 0.35 & 34.85 & 21.79 & 2.27 & 0.13 & 2.21 & 0.02 & 0 & 0 \\
\hline Blattode & но & & & & & & & & & & & & & & & & & & & & & & & & \\
\hline a & 1 & 0 & 0.02 & 0.04 & 0.82 & 0.64 & 0.02 & 0.01 & 0.2 & 0.4 & 0.11 & 5.23 & 0.87 & 0.04 & 0.31 & 0.03 & 0.09 & 3.82 & 10.84 & 1.48 & 0.34 & 1.01 & 0.11 & 0.04 & 0 \\
\hline Blattode & HO & & & & & & & & & & & & & & & & & & & & & & & & \\
\hline \multirow[t]{2}{*}{ a } & 2 & 0 & 0.02 & 0.02 & 0.44 & 0.6 & 0.01 & 0 & 0 & 0.2 & 0.38 & 5.01 & 0.01 & 0.3 & 0.19 & 0.02 & 0 & 13.88 & 33.22 & 0.4 & 0.17 & 0.23 & 0 & 0.01 & 0 \\
\hline & HO & & & & & & & & & & & & & & & & & & & & & & & & \\
\hline Blattode & $3 \&$ & & & & & & & & & & & & & & & & & & & & & & & & \\
\hline \multirow[t]{2}{*}{ a } & 4 & 0 & 0.03 & 0.06 & 0.35 & 0.22 & 0.04 & 0 & 0.09 & 0.28 & 0.26 & 5.85 & 5.32 & 0.17 & 0 & 0.05 & 0.17 & 14.08 & 28.95 & 9.12 & 0.09 & 1.3 & 0.02 & 0 & 0 \\
\hline & & & $0.09 \pm$ & $0.28 \pm$ & $1.74 \pm$ & $1.28 \pm$ & $0.02 \pm$ & $0.02 \pm$ & $0.67 \pm$ & & $0.52 \pm$ & & $0.67 \pm$ & $0.61 \pm$ & $0.35 \pm$ & & $0.31 \pm$ & $13.78 \pm$ & & & $0.33 \pm$ & & & & \\
\hline Chilopod & $\mathrm{HF}$ & & 0.15 & 0.16 & 1.21 & 1.36 & 0.03 & 0.03 & 0.22 & $0.1 \pm 0$. & 0.33 & $1.18 \pm 1$ & 0.37 & 0.39 & 0.33 & $0.07 \pm$ & 0.21 & 2.25 & $24.55 \pm 7$ & $0.94 \pm 0$ & 0.38 & $2.38 \pm 2$ & $0.88 \pm 0.5$ & $0.39 \pm$ & $0 \pm 0$ \\
\hline a & 2 & $\mathrm{~F}$ & (3) & (3) & (3) & (3) & (3) & (3) & (3) & $06(3)$ & (3) & $.02(3)$ & (3) & (3) & (3) & $0.1(3)$ & (3) & (3) & $.99(3)$ & 75 (3) & (3) & 69 (3) & $2(3)$ & $0.6(3)$ & (3) \\
\hline
\end{tabular}


Chapter 2

\begin{tabular}{|c|c|c|c|c|c|c|c|c|c|c|c|c|c|c|c|c|c|c|c|c|c|c|c|c|c|}
\hline & & & & $0.81 \pm$ & & $1.43 \pm$ & & & & $0.39 \pm$ & $0.97 \pm$ & & $0.43 \pm$ & & $0.52 \pm$ & $0.02 \pm$ & $0.17 \pm$ & $12.15 \pm$ & & & & & & $0.22 \pm$ & \\
\hline Chilopod & HO & & $0.6 \pm 0$. & 1.06 & $1.5 \pm 0$. & 0.52 & $0.01 \pm$ & $0 \pm 0$ & $0.12 \pm$ & 0.07 & 0.08 & $5.87 \pm 3$ & 0.18 & $0.45 \pm$ & 0.02 & 0.03 & 0.11 & 3.96 & $26.62 \pm 4$ & $0.75 \pm 0$ & $0.3 \pm 0$ & $3.68 \pm 0$ & $1.15 \pm 0.4$ & 0.11 & $0 \pm 0$ \\
\hline \multirow[t]{2}{*}{ a } & 1 & 0 & $57(2)$ & (2) & $94(2)$ & (2) & $0(2)$ & (2) & $0(2)$ & (2) & (2) & $.26(2)$ & (2) & $0.2(2)$ & (2) & (2) & (2) & (2) & $.75(2)$ & $44(2)$ & $37(2)$ & 35 (2) & $4(2)$ & (2) & (2) \\
\hline & & & $0.11 \pm$ & $0.14 \pm$ & $1.39 \pm$ & $2.05 \pm$ & $0.04 \pm$ & $0.12 \pm$ & & & & & $0.46 \pm$ & & & $0.05 \pm$ & $0.17 \pm$ & & & & $0.23 \pm$ & & & $0.07 \pm$ & $0.01 \pm$ \\
\hline Chilopod & но & & 0.08 & 0.16 & 1.59 & 1.78 & 0.03 & 0.17 & $0.4 \pm 0$ & $0.08 \pm$ & $0.9 \pm 1$ & $2.91 \pm 2$ & 0.16 & $0.61 \pm$ & $0.5 \pm 0$ & 0.08 & 0.08 & $12.6 \pm 2$ & $24.02 \pm 1$ & $1.46 \pm 0$ & 0.38 & $3.75 \pm 2$ & $0.55 \pm 0.7$ & 0.06 & 0.02 \\
\hline a & 2 & 0 & (3) & (3) & (3) & (3) & (3) & (3) & $28(3)$ & $0.1(3)$ & $13(3)$ & $.55(3)$ & (3) & $0.4(3)$ & $66(3)$ & (3) & (3) & $.86(3)$ & $0.93(3)$ & $62(3)$ & (3) & 75 (3) & $8(3)$ & (3) & (3) \\
\hline Chilopod & HR & & & & & & & & & & & & & & & & & & & & & & & & \\
\hline a & 3 & $\mathrm{R}$ & 0.74 & 1.03 & 1.7 & 0.84 & 0.01 & 0.01 & 0.81 & 0.24 & 0.74 & 1.57 & 0.48 & 0.08 & 0.22 & 0.22 & 0.4 & 7.5 & 24.48 & 1.37 & 0 & 7.14 & 2.29 & 0 & 0 \\
\hline Coleopte & $\mathrm{HF}$ & & & & & & & & & & & & & & & & & & & & & & & & \\
\hline ra & 2 & $\mathrm{~F}$ & 0.04 & 0.13 & 0.33 & 1.19 & 0.01 & 0 & 1.15 & 0.03 & 0.13 & 3.22 & 0.55 & 1.93 & 0.87 & 0.08 & 0.19 & 5.35 & 28.75 & 1.49 & 0.06 & 3.49 & 0.45 & 0.25 & 0.02 \\
\hline Coleopte & $\mathrm{HF}$ & & & & & & & & & & & & & & & & & & & & & & & & \\
\hline \multirow[t]{2}{*}{$\mathrm{ra}$} & 4 & $\mathrm{~F}$ & 0.02 & 0.02 & 1.99 & 0.23 & 0 & 0 & 1.83 & 0.16 & 0.64 & 1.63 & 0.81 & 2.27 & 0.29 & 0.09 & 0.34 & 8.06 & 26.3 & 1.95 & 0.05 & 4.32 & 0.36 & 0 & 0 \\
\hline & & & $0.38 \pm$ & $0.41 \pm$ & $0.66 \pm$ & & $0.07 \pm$ & & $0.44 \pm$ & $0.08 \pm$ & $4.36 \pm$ & & $1.77 \pm$ & & $0.47 \pm$ & & $0.12 \pm$ & $18.08 \pm$ & & & $0.82 \pm$ & & & & \\
\hline Coleopte & HR & & 0.53 & 0.51 & 0.93 & $1.48 \pm$ & 0.03 & $0.1 \pm 0$. & 0.01 & 0.11 & 5.19 & $5.86 \pm 4$ & 1.17 & $1.27 \pm$ & 0.66 & $0.8 \pm 1$ & 0.17 & 6.31 & $17.77 \pm 4$ & $0.12 \pm 0$ & 1.16 & $2.8 \pm 2.9$ & $0.26 \pm 0.3$ & $0 \pm 0$ & $0 \pm 0.0$ \\
\hline \multirow[t]{2}{*}{ ra } & 2 & $\mathrm{R}$ & (2) & (2) & (2) & $0.2(2)$ & (2) & $11(2)$ & (2) & (2) & (2) & $.06(2)$ & (2) & $1.8(2)$ & (2) & $12(2)$ & (2) & (2) & $.91(2)$ & 01 (2) & (2) & $5(2)$ & $7(2)$ & (2) & $1(2)$ \\
\hline & & & $1.09 \pm$ & $1.68 \pm$ & $5.33 \pm$ & $9.02 \pm$ & $0.12 \pm$ & $0.01 \pm$ & & & $3.36 \pm$ & & $1.03 \pm$ & $0.94 \pm$ & $1.56 \pm$ & $0.36 \pm$ & & & & & $0.26 \pm$ & & & $0.91 \pm$ & $0.08 \pm$ \\
\hline Collembo & $\mathrm{HF}$ & & 1.68 & 1.01 & 1.41 & 2.98 & 0.09 & 0.02 & $0.35 \pm$ & $0.06 \pm$ & 1.36 & & 1.54 & 0.61 & 1.47 & 0.26 & $0 \pm 0.0$ & $2.81 \pm 1$ & $4.19 \pm 4$. & $0.08 \pm 0$ & 0.23 & $0.4 \pm 0.6$ & $0.42 \pm 0.6$ & 0.68 & 0.12 \\
\hline la & 2 & $\mathrm{~F}$ & (3) & (3) & (3) & (3) & (3) & (3) & $0.3(3)$ & $0.1(3)$ & (3) & $0 \pm 0(3)$ & (3) & (3) & (3) & (3) & $1(3)$ & $.54(3)$ & $88(3)$ & $14(3)$ & (3) & $3(3)$ & $3(3)$ & (3) & (3) \\
\hline Collembo & $\mathrm{HF}$ & & & & & & & & & & & & & & & & & & & & & & & & \\
\hline \multirow[t]{2}{*}{ la } & 4 & $\mathrm{~F}$ & 0.5 & 0.21 & 3.64 & 4.8 & 0.05 & 0.04 & 0.45 & 0.67 & 5.66 & 0 & 0.1 & 1.92 & 0.7 & 0.05 & 0.07 & 5.88 & 11.1 & 0.6 & 0.07 & 2 & 0.04 & 0.96 & 0.04 \\
\hline & HO & & & & & & & & & & & & & & & & & & & & & & & & \\
\hline Collembo & $3 \&$ & & & & & & & & & & & & & & & & & & & & & & & & \\
\hline la & 4 & 0 & 0 & 0.15 & 4.9 & 2.85 & 0.12 & 0 & 0.21 & 0.15 & 6.02 & 0.4 & 0.29 & 0.07 & 0.18 & 0.03 & 0.08 & 2.87 & 17.8 & 0.14 & 0.68 & 1.76 & 1.44 & 0 & 0.16 \\
\hline Collembo & HR & & & & & & & & & & & & & & & & & & & & & & & & \\
\hline \multirow[t]{2}{*}{ la } & 1 & $\mathrm{R}$ & 0.05 & 0.68 & 3.34 & 5.66 & 0.02 & 0.39 & 0.62 & 0 & 5.78 & 0 & 1.99 & 1.7 & 4.38 & 0.03 & 0 & 2.85 & 10.15 & 0.24 & 0.45 & 0.17 & 0 & 0.89 & 0.14 \\
\hline & & & & $0.17 \pm$ & $2.26 \pm$ & $1.06 \pm$ & $0.04 \pm$ & $0.08 \pm$ & $0.54 \pm$ & $0.09 \pm$ & $6.45 \pm$ & & 4.17士 & & $0.54 \pm$ & & $0.02 \pm$ & & & & & & & $0.15 \pm$ & \\
\hline Collembo & HR & & $0.2 \pm 0$ & 0.19 & 2.54 & 0.95 & 0.04 & 0.04 & 0.39 & 0.15 & 0.41 & $4.63 \pm 3$ & 5.05 & $0.2 \pm 0$. & 0.79 & $0.65 \pm$ & 0.03 & $7.14 \pm 4$ & $15.18 \pm 0$ & $0.74 \pm 0$ & $0.76 \pm$ & $1.78 \pm 1$. & $0.61 \pm 1.0$ & 0.25 & $0 \pm 0$ \\
\hline la & 2 & $\mathrm{R}$ & $27(3)$ & (3) & (3) & (3) & (3) & (3) & (3) & (3) & (3) & $.83(3)$ & (3) & $18(3)$ & (3) & $0.4(3)$ & (3) & $.08(3)$ & $.65(3)$ & 77 (3) & $1(3)$ & $49(3)$ & $4(3)$ & (3) & (3) \\
\hline
\end{tabular}


Chapter 2

\begin{tabular}{|c|c|c|c|c|c|c|c|c|c|c|c|c|c|c|c|c|c|c|c|c|c|c|c|c|c|}
\hline & & & & $0.02 \pm$ & $0.17 \pm$ & $0.18 \pm$ & $0.02 \pm$ & & $0.04 \pm$ & & $0.31 \pm$ & $11.61 \pm$ & $0.27 \pm$ & $0.08 \pm$ & $0.02 \pm$ & $0.01 \pm$ & & & & $20.14 \pm$ & $0.09 \pm$ & & & $0.03 \pm$ & \\
\hline Dermapt & HO & & $0 \pm 0$ & 0.01 & 0.08 & 0.08 & 0.01 & $0.01 \pm$ & 0.06 & $0.1 \pm 0$ & 0.04 & 7.75 & 0.35 & 0.01 & 0.01 & 0.01 & $0.02 \pm$ & $4.61 \pm 0$ & $15.23 \pm 0$ & 27.59 & 0.04 & $0.31 \pm 0$ & $0.01 \pm 0.0$ & 0.01 & $0 \pm 0$ \\
\hline era & 1 & 0 & (2) & (2) & (2) & (2) & (2) & $0(2)$ & (2) & $02(2)$ & (2) & (2) & (2) & (2) & (2) & (2) & 0 (2) & $.6(2)$ & $.08(2)$ & (2) & (2) & $33(2)$ & $1(2)$ & (2) & (2) \\
\hline Dermapt & HO & & & & & & & & & & & & & & & & & & & & & & & & \\
\hline \multirow[t]{2}{*}{ era } & 2 & 0 & 0.03 & 0.05 & 0.9 & 1.07 & 0.01 & 0 & 0.06 & 0.26 & 0.26 & 8.01 & 0.22 & 0.28 & 0.43 & 0 & 0.09 & 7.67 & 29.34 & 0.91 & 0.13 & 0.25 & 0.29 & 0 & 0 \\
\hline & $\mathrm{HF}$ & & & & & & & & & & & & & & & & & & & & & & & & \\
\hline Diplopod & $1 \&$ & & & & & & & & & & & & & & & & & & & & & & & & \\
\hline \multirow[t]{2}{*}{ a } & 3 & $\mathrm{~F}$ & 0.03 & 0.86 & 3.37 & 5.83 & 0 & 0.07 & 0.24 & 0 & 1.66 & 0 & 2.38 & 1.23 & 4.61 & 0 & 0 & 5.35 & 6.95 & 3.35 & 0.68 & 0.65 & 0 & 0.89 & 0.22 \\
\hline & & & & & & $1.61 \pm$ & $0.06 \pm$ & $0.04 \pm$ & $0.89 \pm$ & $0.62 \pm$ & $0.71 \pm$ & & & $1.14 \pm$ & & $0.08 \pm$ & & & & & $1.13 \pm$ & & & $0.92 \pm$ & $1.94 \pm$ \\
\hline Diplopod & $\mathrm{HF}$ & & $0.02 \pm$ & $0.2 \pm 0$ & $1.8 \pm 0$. & 0.91 & 0.02 & 0.04 & 0.03 & 0.16 & 0.37 & $1.94 \pm 2$ & $1.4 \pm 0$. & 0.22 & $0.3 \pm 0$ & 0.08 & $0.17 \pm$ & $14.5 \pm 3$ & $19.69 \pm 9$ & $4.48 \pm 1$. & 1.17 & $1.53 \pm 0$ & & 0.85 & 2.55 \\
\hline \multirow[t]{2}{*}{ a } & 2 & $\mathrm{~F}$ & $0(2)$ & $01(2)$ & $47(2)$ & (2) & (2) & (2) & (2) & (2) & (2) & $.41(2)$ & $66(2)$ & (2) & $12(2)$ & (2) & $0.1(2)$ & $.3(2)$ & $.03(2)$ & $75(2)$ & (2) & $54(2)$ & $0 \pm 0$ (2) & (2) & (2) \\
\hline & & & $0.02 \pm$ & $0.02 \pm$ & $0.79 \pm$ & $0.63 \pm$ & & & $0.21 \pm$ & $0.16 \pm$ & $0.25 \pm$ & & $0.62 \pm$ & $0.52 \pm$ & & $0.02 \pm$ & $0.13 \pm$ & $10.28 \pm$ & & & $0.12 \pm$ & & & & $0.02 \pm$ \\
\hline Diplopod & HO & & 0.03 & 0.01 & 0.14 & 0.25 & $0.01 \pm$ & $0 \pm 0.0$ & 0.02 & 0.06 & 0.12 & $3.42 \pm 1$ & 0.28 & 0.26 & $0.2 \pm 0$ & 0.02 & 0.02 & 1.86 & $27.58 \pm 5$ & $0.91 \pm 0$ & 0.08 & $1.14 \pm 0$ & $0.23 \pm 0.2$ & $0.1 \pm 0$. & 0.02 \\
\hline a & 1 & 0 & (4) & (4) & (4) & (4) & $0(4)$ & $1(4)$ & (4) & (4) & (4) & $.76(4)$ & (4) & (4) & $14(4)$ & (4) & (4) & (4) & $.3(4)$ & $96(4)$ & (4) & $53(4)$ & (4) & $11(4)$ & (4) \\
\hline Diplopod & HR & & & & & & & & & & & & & & & & & & & & & & & & \\
\hline a & 2 & $\mathrm{R}$ & 0.81 & 1.08 & 1.68 & 1.67 & 0.02 & 0.06 & 0.84 & 0.15 & 3.8 & 0.07 & 0.28 & 0.57 & 0.73 & 0.05 & 0.33 & 14.3 & 27.98 & 3.21 & 0.58 & 1 & 0.59 & 0.04 & 0.03 \\
\hline Diplopod & HR & & & & & & & & & & & & & & & & & & & & & & & & \\
\hline \multirow[t]{2}{*}{ a } & 4 & $\mathrm{R}$ & 0.03 & 0.2 & 8.9 & 0.34 & 0.05 & 0.04 & 0.36 & 0.18 & 2.56 & 0.02 & 0.26 & 0 & 0.27 & 0.5 & 12.66 & 13.59 & 10.36 & 0.11 & 0 & 1.86 & 0.02 & 0.05 & 0 \\
\hline & $\mathrm{HF}$ & & & & & & & & & & & & & & & & & & & & & & & & \\
\hline \multirow[t]{3}{*}{ Isopoda } & 4 & $\mathrm{~F}$ & 1.82 & 0.67 & 3.88 & 7.53 & 0.04 & 0 & 0.32 & 1.01 & 2.99 & 0 & 1.93 & 0.73 & 4.9 & 0.53 & 0.02 & 4.76 & 5.27 & 0.49 & 1.95 & 0.57 & 0.3 & 0 & 0.2 \\
\hline & & & & $0.08 \pm$ & $1.18 \pm$ & & $0.02 \pm$ & & $0.66 \pm$ & $0.28 \pm$ & & & $1.35 \pm$ & $0.62 \pm$ & $0.26 \pm$ & $0.06 \pm$ & $0.15 \pm$ & $12.98 \pm$ & & & & & & $0.15 \pm$ & \\
\hline & HO & & $0.01 \pm$ & 0.08 & 0.43 & $0.4 \pm 0$ & 0.01 & $0 \pm 0$ & 0.09 & 0.06 & $0.3 \pm 0$ & $6.84 \pm 0$ & 0.01 & 0.33 & 0.09 & 0.08 & 0.03 & 0.74 & $21.03 \pm 2$ & $2.56 \pm 0$ & $0.4 \pm 0$ & $2.29 \pm 0$ & $0.46 \pm 0.1$ & 0.12 & $0 \pm 0$ \\
\hline \multirow[t]{2}{*}{ Isopoda } & 1 & 0 & $0(2)$ & (2) & (2) & $17(2)$ & (2) & (2) & (2) & (2) & $01(2)$ & $.71(2)$ & (2) & (2) & (2) & (2) & (2) & (2) & $.74(2)$ & $62(2)$ & $46(2)$ & $37(2)$ & $5(2)$ & (2) & (2) \\
\hline & HO & & & & & & & & & & & & & & & & & & & & & & & & \\
\hline Isopoda & 2 & 0 & 1.07 & 0.37 & 4.74 & 4.76 & 0.03 & 0 & 0.34 & 1.18 & 0.84 & 2.02 & 0.63 & 3.02 & 2.4 & 0.53 & 0.12 & 3.95 & 10.41 & 0.21 & 2.91 & 0 & 0 & 1.05 & 0 \\
\hline Lepidopt & но & & & & & & & & & & & & & & & & & & & & & & & & \\
\hline \multirow[t]{2}{*}{ era } & 2 & 0 & 0.02 & 0.01 & 0.75 & 0.4 & 0.01 & 0.02 & 0 & 0.16 & 1.16 & 3.49 & 0.07 & 0.04 & 0.07 & 0.01 & 0 & 9.93 & 40.64 & 0.05 & 0.13 & 0.11 & 0 & 0.01 & 0 \\
\hline & $\mathrm{HF}$ & & $0.03 \pm$ & $0.36 \pm$ & & $2.55 \pm$ & $0.01 \pm$ & $0.23 \pm$ & $1.57 \pm$ & $0.02 \pm$ & $0.72 \pm$ & & $1.03 \pm$ & $1.18 \pm$ & $0.67 \pm$ & $0.59 \pm$ & $0.19 \pm$ & $13.55 \pm$ & & & & & & $0.83 \pm$ & $0.04 \pm$ \\
\hline Lumbrici & 1\& & & 0.01 & 0.24 & $2.5 \pm 1$ & 1.07 & 0.02 & 0.24 & 1.11 & 0.04 & 0.65 & & 0.62 & 1.02 & 0.94 & 0.52 & 0.28 & 3.21 & $4.34 \pm 1$ & $4.59 \pm 1$ & $1.4 \pm 1$. & $16 \pm 6.9$ & $3.69 \pm 2.4$ & 0.44 & 0.04 \\
\hline na & 3 & $\mathrm{~F}$ & (3) & (3) & $04(3)$ & (3) & (3) & (3) & (3) & (3) & (3) & $0 \pm 0(3)$ & (3) & (3) & (3) & (3) & (3) & (3) & $25(3)$ & $57(3)$ & $75(3)$ & (3) & $6(3)$ & (3) & (3) \\
\hline
\end{tabular}


Chapter 2

HO

Lumbrici 3

\begin{tabular}{|c|c|c|c|c|c|c|c|c|c|c|c|c|c|c|c|c|c|c|c|c|c|c|c|c|c|}
\hline \multirow{2}{*}{ na } & \multirow[t]{2}{*}{4} & \multirow[t]{2}{*}{0} & \multirow{2}{*}{$\begin{array}{r}0.3 \\
0.09+\end{array}$} & \multirow{2}{*}{$\begin{array}{r}0.09 \\
0.01 \pm\end{array}$} & \multirow{2}{*}{$\begin{array}{r}2.43 \\
2.78 \pm\end{array}$} & \multirow{2}{*}{$\begin{array}{r}1.6 \\
2.02 \pm\end{array}$} & \multirow[t]{2}{*}{0.02} & 0.06 & \multirow{2}{*}{$\begin{array}{r}1.9 \\
1.54 \pm\end{array}$} & \multirow{2}{*}{$\begin{array}{c}0.31 \\
0.11 \pm\end{array}$} & \multirow{2}{*}{$\begin{array}{r}1.81 \\
0.84 \pm\end{array}$} & \multirow[t]{2}{*}{0} & \multirow{2}{*}{$\begin{array}{r}1.34 \\
1.23 \pm\end{array}$} & \multirow{2}{*}{$\begin{array}{r}0.28 \\
0.37 \pm\end{array}$} & \multirow{2}{*}{$\begin{array}{c}0 \\
0.22 \pm\end{array}$} & \multirow{2}{*}{$\begin{array}{r}0.45 \\
0.28 \pm\end{array}$} & \multirow{2}{*}{$\begin{array}{c}0.07 \\
0.14 \pm\end{array}$} & \multirow[t]{2}{*}{12.85} & \multirow[t]{2}{*}{6.68} & \multirow[t]{2}{*}{0.09} & \multirow[t]{2}{*}{3.06} & 14.69 & 2.75 & 0.01 & 0.02 \\
\hline & & & & & & & & $0.06 \pm$ & & & & & & & & & & & & & & & & $0.02 \pm$ & $0.08 \pm$ \\
\hline umbrici & HR & & 0.07 & 0.01 & 0.08 & 2.12 & $0 \pm 0.0$ & 0.04 & 0.31 & 0.01 & 1.19 & $0.68 \pm 0$ & 0.56 & 0.42 & 0.11 & 0.39 & 0.18 & $15.43 \pm$ & $6.87 \pm 0$ & $3.68 \pm 0$ & $0.2 \pm 0$ & $21.51 \pm$ & $5.59 \pm 1.6$ & 0.04 & 0.06 \\
\hline na & 2 & $\mathrm{R}$ & (2) & (2) & (2) & (2) & $1(2)$ & (2) & (2) & (2) & (2) & $.96(2)$ & (2) & (2) & (2) & (2) & (2) & $1.8(2)$ & $3(2)$ & $3(2)$ & $28(2)$ & $5.11(2)$ & $8(2)$ & (2) & (2) \\
\hline Lumbrici & HR & & & & & & & & & & & & & & & & & & & & & & & & \\
\hline na & 3 & $\mathrm{R}$ & 0.9 & 0.84 & 3.78 & 1.04 & 0.03 & 0.16 & 1.76 & 0.13 & 0 & 0.17 & 0.79 & 1.54 & 0.31 & 0.35 & 0.18 & 13.37 & 5.34 & 4.13 & 0.56 & 20.3 & 4.55 & 0.25 & 0 \\
\hline Mesostig & $\mathrm{HF}$ & & & & & & & & & & & & & & & & & & & & & & & & \\
\hline mata & 2 & $\mathrm{~F}$ & 0.59 & 0.67 & 3.24 & 6.33 & 0.13 & 0 & 0.45 & 0 & 2.93 & 0 & 1.61 & 6.23 & 4.05 & 0.26 & 0.05 & 9.25 & 13.87 & 0.89 & 0 & 2.03 & 0.64 & 0.05 & 0.17 \\
\hline Mesostig & HO & & & & & & & & & & & & & & & & & & & & & & & & \\
\hline mata & 1 & 0 & 0.09 & 0.11 & 4.29 & 2.51 & 0.03 & 0 & 0.28 & 0 & 2.12 & 1.05 & 0.6 & 0 & 0.16 & 0.13 & 0.01 & 7.62 & 26.11 & 0.83 & 2.02 & 1.66 & 0 & 0 & 0 \\
\hline & & & & & $4.67 \pm$ & $2.83 \pm$ & & $0.08 \pm$ & $0.35 \pm$ & & $2.38 \pm$ & & $0.48 \pm$ & & $0.67 \pm$ & & $0.02 \pm$ & & & & $0.41 \pm$ & & & & \\
\hline Mesostig & HO & & $1.5 \pm 2$ & $2.8 \pm 3$ & 5.93 & 3.11 & $0.29 \pm$ & 0.08 & 0.01 & $0.4 \pm 0$ & 0.79 & $1.18 \pm 0$ & 0.18 & $0.9 \pm 1$. & 0.16 & $0.2 \pm 0$ & 0.02 & $6.7 \pm 3$. & $16.76 \pm 1$ & $1.03 \pm 0$ & 0.44 & $2.19 \pm 1$ & $0.42 \pm 0.1$ & $0.72 \pm$ & $0 \pm 0$ \\
\hline mata & 2 & 0 & 09 (2) & $27(2)$ & (2) & (2) & $0.4(2)$ & (2) & (2) & $49(2)$ & (2) & $.35(2)$ & (2) & $27(2)$ & (2) & $23(2)$ & (2) & $74(2)$ & $3.82(2)$ & $1(2)$ & (2) & 48 (2) & $3(2)$ & $0.9(2)$ & (2) \\
\hline & $\mathrm{HF}$ & & & & & & & & & & & & & & & & & & & & & & & & \\
\hline Opiliones & 4 & $\mathrm{~F}$ & 0.21 & 0.15 & 3.92 & 8.81 & 0.07 & 0.02 & 0.32 & 0.01 & 0 & 0 & 0.46 & 3.12 & 0.97 & 0.1 & 0.01 & 4.96 & 10.92 & 0.82 & 1.32 & 1.98 & 0.2 & 0.31 & 0.02 \\
\hline & HO & & & & & & & & & & & & & & & & & & & & & & & & \\
\hline Opiliones & 1 & 0 & 3.22 & 3.93 & 7.55 & 6.53 & 0.05 & 0 & 0.16 & 0.98 & 1.13 & 0 & 0.05 & 1.77 & 2.91 & 0.36 & 0.02 & 4.64 & 6.01 & 0.18 & 2.06 & 1.49 & 1.13 & 1.27 & 0.12 \\
\hline & HR & & & & & & & & & & & & & & & & & & & & & & & & \\
\hline Opiliones & 2 & $\mathrm{R}$ & 0.01 & 0.1 & 4.73 & 2.87 & 0.05 & 0.16 & 0.7 & 0.18 & 0.17 & 0.86 & 0 & 0.44 & 0 & 0.02 & 0.15 & 11.87 & 13.54 & 2.67 & 0 & 4.96 & 0.08 & 0.07 & 0 \\
\hline & $\mathrm{HF}$ & & & & & & & & & & & & & & & & & & & & & & & & \\
\hline Oribatida & 2 & $\mathrm{~F}$ & 0.07 & 0.69 & 3.4 & 6.72 & 0.1 & 0 & 0.96 & 0.47 & 3.43 & 0 & 0.82 & 1 & 0.32 & 0.6 & 0 & 15.65 & 8.53 & 0 & 0.43 & 0.01 & 1.08 & 0.21 & 0.13 \\
\hline & $\mathrm{HF}$ & & & & & & & & & & & & & & & & & & & & & & & & \\
\hline Oribatida & 4 & $\mathrm{~F}$ & 3.71 & 3.55 & 5.47 & 7.45 & 0.03 & 0.01 & 0.21 & 0.93 & 2.48 & 0 & 1.4 & 0.15 & 0.04 & 0.3 & 0.01 & 4.6 & 5.05 & 0 & 3.31 & 0 & 0.35 & 0.54 & 0.14 \\
\hline & HO & & & & & & & & & & & & & & & & & & & & & & & & \\
\hline Oribatida & 2 & 0 & 0 & 0.37 & 0.15 & 4.97 & 0.16 & 0.21 & 0.11 & 0.23 & 4.57 & 1.93 & 1.9 & 0 & 0 & 0.09 & 0.01 & 7.32 & 15.81 & 0 & 0.12 & 0.97 & 0 & 0.01 & 0.04 \\
\hline & HR & & & & & & & & & & & & & & & & & & & & & & & & \\
\hline & 18 & & & & & & & & & & & & & & & & & & & & & & & & \\
\hline Oribatida & 3 & R & 0 & 0.14 & 1.51 & 2.58 & 0.12 & 0.2 & 0.1 & 0.13 & 2.69 & 0.06 & 0.17 & 0.69 & 0 & 0 & 0.36 & 1.86 & 8.13 & 0.43 & 0.13 & 1.13 & 0 & 0.03 & 10.8 \\
\hline
\end{tabular}


Chapter 2

\begin{tabular}{|c|c|c|c|c|c|c|c|c|c|c|c|c|c|c|c|c|c|c|c|c|c|c|c|c|c|}
\hline Oribatida & 2 & $\mathrm{R}$ & 0.21 & 0.25 & 0.98 & 0.87 & 0.05 & 0.1 & 0 & 0.4 & 5.75 & 6.13 & 0.28 & 0.87 & 0 & 0.35 & 0 & 15.72 & 26.59 & 0.16 & 0.02 & 0 & 0 & 0 & 0 \\
\hline $\begin{array}{l}\text { Pseudosc } \\
\text { orpionid }\end{array}$ & $\mathrm{HF}$ & & & & & & & & & & & & & & & & & & & & & & & & \\
\hline a & 2 & $\mathrm{~F}$ & 0.17 & 0.09 & 2.99 & 3.86 & 0.04 & 0 & 0.48 & 0.18 & 1.95 & 1.07 & 0.74 & 0.24 & 0.77 & 0.52 & 0.24 & 8.11 & 16.29 & 2.17 & 0.81 & 4.48 & 1.34 & 0 & 0.11 \\
\hline \multicolumn{26}{|l|}{ Pseudosc } \\
\hline orpionid & $\mathrm{HR}$ & & & & & & & & & & & & & & & & & & & & & & & & \\
\hline a & 4 & $\mathrm{R}$ & 1.39 & 1.07 & 2.68 & 1.38 & 0.02 & 0.04 & 1.14 & 0.11 & 0.99 & 2.19 & 0.35 & 0.07 & 0.01 & 0.11 & 0.37 & 11.11 & 23.82 & 2.18 & 0 & 6 & 1.91 & 0.5 & 0 \\
\hline Psocopte & $\mathrm{HR}$ & & & & & & & & & & & & & & & & & & & & & & & & \\
\hline $\mathrm{ra}$ & 2 & $\mathrm{R}$ & 3.26 & 1.82 & 1.14 & 1.32 & 0 & 0 & 0.27 & 0.61 & 0.72 & 0.97 & 0.31 & 0.57 & 0.48 & 0 & 0.33 & 20.59 & 16.88 & 0.97 & 0.31 & 0.13 & 0 & 0.46 & 0 \\
\hline Schizomi & $\mathrm{HF}$ & & & & & & & & & & & & & & & & & & & & & & & & \\
\hline da & 4 & $\mathrm{~F}$ & 0.05 & 0.16 & 1.48 & 7.01 & 0 & 0.63 & 0 & 0 & 2.21 & 0 & 3.11 & 4.73 & 3.72 & 0 & 0 & 10.41 & 13.57 & 0.79 & 1.52 & 9.39 & 1.98 & 0.11 & 0.02 \\
\hline Schizomi & $\mathrm{HR}$ & & & & & & & & & & & & & & & & & & & & & & & & \\
\hline \multirow[t]{2}{*}{ da } & 2 & $\mathrm{R}$ & 0.33 & 0.15 & 4.55 & 2.94 & 0.03 & 0 & 0.62 & 0.27 & 0 & 1.49 & 0.42 & 0 & 0.19 & 0.05 & 0.2 & 12.52 & 14.04 & 0.54 & 0 & 0.12 & 1.7 & 0.27 & 0 \\
\hline & $\mathrm{HF}$ & & & & & & & & & & & & & & & & & & & & & & & & \\
\hline Symphyl & $1 \&$ & & & & & & & & & & & & & & & & & & & & & & & & \\
\hline \multirow[t]{2}{*}{ a } & 3 & $\mathrm{~F}$ & 0.01 & 0.01 & 0.02 & 1.48 & 0.03 & 0.04 & 3.03 & 0.16 & 4.97 & 5.58 & 0.83 & 1.18 & 1.18 & 0.13 & 0.12 & 7.11 & 27.03 & 0.77 & 0.23 & 2.12 & 0.08 & 0.5 & 0.19 \\
\hline & Hо & & & & & & & & & & & & & & & & & & & & & & & & \\
\hline Symphyl & $3 \&$ & & & & & & & & & & & & & & & & & & & & & & & & \\
\hline a & 4 & 0 & 0 & 0.07 & 4.75 & 2.49 & 0 & 0.06 & 0.25 & 0.16 & 0.71 & 2.63 & 0.25 & 0 & 0.52 & 0.07 & 0.13 & 3.37 & 21.63 & 1.92 & 0 & 0.04 & 0 & 0.01 & 0.05 \\
\hline Symphyl & $\mathrm{HR}$ & & & & & & & & & & & & & & & & & & & & & & & & \\
\hline a & 2 & $R$ & 4.01 & 6.78 & 9.61 & 5.75 & 0.06 & 0.01 & 0.43 & 0.26 & 0.98 & 0.05 & 0 & 0 & 0 & 0.35 & 0.02 & 2.96 & 5.28 & 0.18 & 2.07 & 0.41 & 0.01 & 0.13 & 0 \\
\hline
\end{tabular}


Chapter 2 
CHAPTER 3

CONVERSION OF RAINFOREST INTO OIL PALM AND RUBBER PLANTATIONS AFFECTS THE FUNCTIONAL COMPOSITION OF LITTER AND SOIL COLLEMBOLA

Winda Ika Susanti, Tamara Bartels, Valentyna Krashevska, Rahayu Widyastuti, Louis Deharveng, Stefan Scheu, Anton Potapov
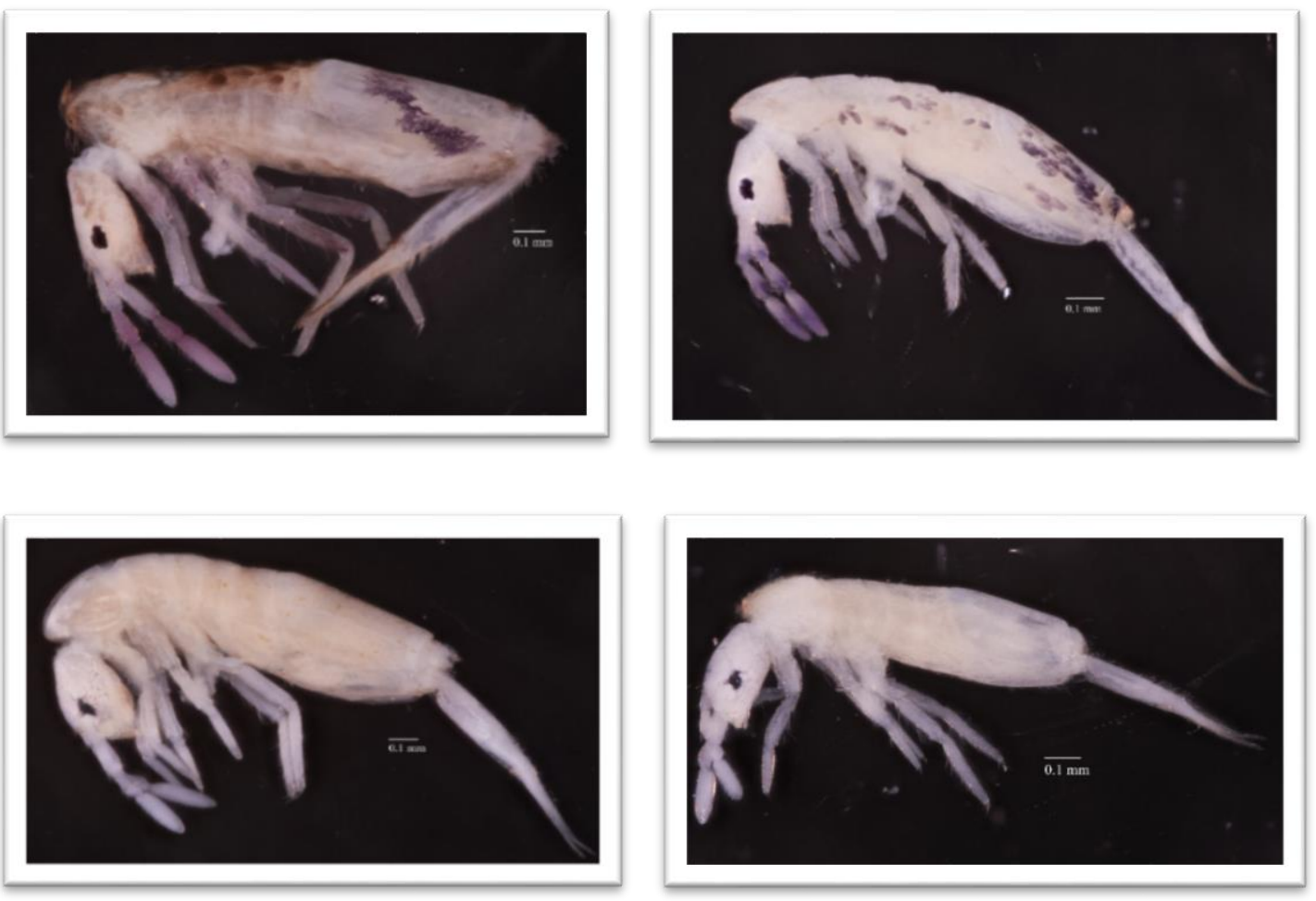

Photos by Winda Ika Susanti 


\title{
CONVERSION OF RAINFOREST INTO OIL PALM AND RUBBER PLANTATIONS AFFECTS THE FUNCTIONAL COMPOSITION OF LITTER AND SOIL COLLEMBOLA
}

\begin{abstract}
Rainforest conversion and expansion of plantations in tropical regions are associated with changes in animal communities and biodiversity decline. In soil, Collembola are one of the most numerous invertebrate groups that affect the functioning of microbial communities and support arthropod predators. Despite that, information on the impact of changes in land use in the tropics on species and trait composition of Collembola communities is very limited. We investigated the response of Collembola to the conversion of rainforest into rubber agroforestry ('jungle rubber'), rubber and oil palm plantations in Jambi province (Sumatra, Indonesia), a region which experienced one of the strongest recent deforestation globally. Collembola were sampled in 2013 and 2016 from the litter and soil layer using heat extraction, and environmental factors were measured (litter $\mathrm{C} / \mathrm{N}$ ratio and amount, $\mathrm{pH}$, water content, composition of microbial community and predator abundance). In the litter layer, density and species richness in plantation systems were $25-38 \%$ and $30-40 \%$ lower, respectively, than in rainforest. However, in the soil layer, density, species richness and trait diversity of Collembola were only slightly affected by land-use change, contrasting the response of many other animal groups. Species and trait composition of Collembola communities in litter and soil differed between all land-use systems. Water content and $\mathrm{pH}$ were identified as main factors related to the differences in species and trait composition both in litter and soil, followed by the density of micro- and macropredators. Dominant species of Collembola in rainforest and jungle rubber were characterized by small body size, absence of furca and absent or intense pigmentation, while in plantations larger species with long furca and diffuse or patterned coloration were more abundant. Overall, land-use change negatively affected Collembola communities in the litter layer, but their impact was lower in the soil layer. Several pantropical genera of Collembola (i.e., Isotomiella, Pseudosinella and Folsomides) dominated across land-use systems, reflecting their high environmental adaptability or efficient dispersal calling for studies on their ecology and genetic diversity. The decline in species richness and density of litter-dwelling Collembola with the conversion of rainforest into plantation systems calls for
\end{abstract}


management practices mitigating negative effects of the deterioration of the litter layer in rubber plantations, but even more in oil palm plantations.

Keywords: springtail, community structure, trait composition, rainforest conversion, agricultural plantation

\section{Introduction}

Agricultural intensification in Indonesia is associated with deforestation, which has increased greatly in the last 30 years and is predicted to continue (Koh and Ghazoul, 2010; Gatto et al., 2015). The conversion of tropical rainforest into plantations is associated with the degradation and destruction of habitats, which is among the most significant and immediate threats to biodiversity worldwide (Titeux et al., 2016). Environmental changes and habitat degradation eliminate or alter ecological niches resulting in a loss of biodiversity and associated changes in ecosystem functioning (Fitzherbert et al., 2008; Gilbert, 2012; Barnes et al., 2014; Clough et al., 2016). Experiencing massive deforestation in the last decades, Sumatra represents a model region for investigating the effect of rainforest conversion into plantation systems on biodiversity and ecosystem functioning at local and regional scales (Clough et al., 2016; Drescher et al., 2016).

Lowland rainforest in Jambi province, Sumatra, has been converted in large into oil palm (16\% of total area) and rubber plantations (12\%) (Gatto et al., 2015). Conversion of rainforest into oil palm and rubber plantations is associated with a strong decline in plant and animal diversity above the ground (Fitzherbert et al., 2008; Rembold et al., 2017; Clough et al., 2016), but also alters soil habitats and detrimentally affects soilassociated biodiversity, as shown for nematodes (Krashevska et al., 2019), testate amoebae (Krashevska et al., 2016), ground spiders (Potapov et al., 2020) and litter macroinvertebrates (Barnes et al., 2014). Extensive compared to intensive management of agricultural systems, such as rubber plantations, however, can mitigate alterations in community composition and negative effects of the conversion of rainforest into agricultural production systems on biodiversity (Nazarreta et al., 2020; Schulz et al., 2019; Krashevska et al., 2016). However, information on effects of the conversion of rainforest into monoculture plantations and agroforestry systems is lacking to a large extent to validate such assumptions regarding many groups of the soil fauna, including Collembola. 
Collembola is a dominant group of soil animals globally, being very abundant in forest soils (Hopkin, 1997; Rusek, 1998; Devi et al., 2011). Collembola significantly affect soil microbial communities, nutrient cycling and soil fertility by feeding on soil microorganisms and dead organic matter (Rusek, 1998; Coulibaly et al., 2019; Potapov et al., 2020). In particular, the presence of different ecological groups of Collembola (soil or surface dwellers) can affect microbial-driven ecosystem processes, such as decomposition and nutrient cycling (Coulibaly et al., 2019). In temperate regions, agricultural intensification (Sousa et al., 2006) and forest plantations (Deharveng 1996) typically are associated with a decrease in species richness and density, as well as a strong alteration in the community composition of Collembola as compared to natural forest. The few studies existing from tropical regions suggest that effects are similar as shown e.g., for monoculture coffee plantations in Mexico (Rojas et al., 2009), and rubber and peach plantations in Amazonia (Martius et al., 2004). However, very few studies investigated the impact of land-use change on Collembola in other tropical regions, including Southeast Asia. A study from Sumatra indicated that the conversion of rainforest into oil palm and rubber plantations negatively affects Collembola density and genus-level diversity and composition (Widrializa et al., 2015), but consequences for the functional diversity and species composition remained unexplored.

Since the biology of tropical soil invertebrates is poorly studied, their functional roles and responses might be inferred from approximations, with functional traits (properties of species that govern their effects on or response to their environment) being a promising approach (Violle et al., 2007; Pey et al., 2014). Trait-based approaches have been suggested to be more advantageous than species-based approaches since they do provide more mechanistic and generalizable links between organisms and their environment (Gagic et al., 2015). Collembola species display a wide variation in traits that can provide a functional and insightful tool for assessing their response to land-use changes (Van Straalen et al., 2008). Traits usually considered for Collembola are morphological characters assumed to be connected to adaptations to environmental conditions. They often are combined into composite traits such as 'life forms', statistically linked to particular habitats, i.e. size of furca, number of ocelli, length of antennae, pigmentation and presence of scales (Vandewalle et al., 2010; Salmon and Ponge, 2012). Collembola species and communities show trait-related responses to a variety of environmental factors, like changes in soil chemistry, microhabitat configuration, vegetation cover and agricultural practices, but primarily Collembola traits reflect the soil 
water content and pH (Chagnon et al., 2001; Chauvat et al., 2007; Son et al., 2009; De Boer et al., 2012). However, it remains unknown which factors drive changes in species and trait composition of Collembola communities in tropical rainforests and plantation systems.

In this study, we investigated species and trait composition of Collembola communities from litter and soil layers in rainforest, rubber agroforestry ('jungle rubber'), rubber and oil palm plantations, in Jambi province, Sumatra, Indonesia. Based on previous studies on the impact of land-use changes on invertebrate communities (Barnes et al., 2014; Krashevska et al., 2016; Krashevska et al., 2019; Potapov et al., 2020), we hypothesized that (1) rainforest and jungle rubber have higher total density, species richness and functional diversity of Collembola than monoculture plantations, (2) community composition of Collembola changes with land use, with the changes being most pronounced between more natural ecosystems (rainforest and jungle rubber) and more intensively used ecosystems (oil palm and rubber plantations), (3) similar to temperate ecosystems, $\mathrm{pH}$ and water content are the most important factors correlated with the composition of Collembola in rainforest and plantations systems, and (4) functional trait composition of Collembola communities varies more predictably with environmental factors than species composition because species turnover across sites is governed by environment-specific trait responses favouring certain functional groups of species.

\section{Material and Methods}

\section{Site description}

The study was conducted in the framework of the EFForTS project investigating in a comprehensive way ecological and socioeconomic changes associated with the transformation of rainforest into plantation systems (http://www.unigoettingen.de/en/310995.html). Soil and litter samples were taken in lowland rainforest, jungle rubber, rubber (Hevea brasiliensis) plantations and oil palm (Elaeis guineensis) plantations, located in Jambi province, southwest Sumatra, Indonesia. The climate is tropical and humid with moderate variations between the rainy season from October to April and the dry season from June to September; between 1991 and 2011 mean annual temperature was $26.7 \pm 0.2^{\circ} \mathrm{C}$ and mean annual precipitation $2235 \pm 381 \mathrm{~mm}$ (Drescher et al., 2016). The study sites were located at similar altitude varying from 50 to $100 \mathrm{~m}$ 
a.s.l. in two landscapes, Harapan and Bukit Duabelas region; the overall area where sampling sites were distributed was about $80 \mathrm{~km}$ in diameter with the distance between sites in the two regions being $<40 \mathrm{~km}$ (for more details see Drescher et al., 2016). Rainforest was included to represent baseline conditions allowing to evaluate changes due to the conversion into agricultural plantations. Except in jungle rubber, samples from upland and riparian sites (being at least partly flooded during the rainy season) were studied. Soils in the Harapan region mainly comprise loamy Acrisols of low fertility, whereas in the Bukit Duabelas region the major soil type is clay Acrisol (Allen et al., 2015; Kotowska et al., 2015). Two regions, and upland and riparian sites were included in the analysis to increase the variation in environmental factors considered (water regimes, soil types) and thereby the generality of our results.

Rubber and oil palm plantations were intensively managed monocultures of 6 to 16 and 8 to 15 years, respectively (Drescher et al., 2016). Typically, oil palm plantations were established after clearing and burning of jungle rubber, whereas rubber plantations were established after logging of rainforest (Allen et al., 2015). Rubber and oil palm plantations were fertilized once in the rainy season and once in the dry season with NPK complete fertilizer (i.e., Phonska and Mahkota), potassium chloride $(\mathrm{KCl})$ and urea $\left(\mathrm{CO}\left(\mathrm{NH}_{2}\right)_{2}\right)$. Additionally, manual and chemical weeding took place throughout the year in both rubber and oil palm plantations. The most commonly used herbicides were Gramoxone and Roundup, applied at an average rate of 2-5 $\mathrm{L} \mathrm{ha}^{-1} \mathrm{y}^{-1}$ (Allen et al., 2015; Kotowska et al., 2015; Clough et al., 2016). More details on management practices of the studied smallholder monoculture plantations are described in Allen et al. (2015).

\section{Sampling procedure}

Samples were taken in October 2013 and October 2016 within $50 \times 50 \mathrm{~m}^{2}$ plots established at each study site (see Drescher et al., 2016) with a distance of ca. 500 meters minimum, but usually more than 1 kilometer, between the plots. Two different years were used as temporal replicates increasing the variability of environmental factors considered and therefore the generality of our results. In 2013, samples were taken in natural rainforest, jungle rubber, rubber and oil palm plantations in two regions with four replicates each, resulting in 32 plots in total (2 regions $\mathrm{x} 4$ land-use systems $\mathrm{x} 4$ replicates). In 2016, samples were taken in rainforest, rubber and oil palm plantations in two regions (2 regions x 3 land-use systems x 4 replicates) with additional rainforest, 
rubber and oil palm plantations in riparian sites of the Harapan region with four replicates each (1 region x 3 land-use systems x 4 replicates), resulting in 36 plots in total (Fig. 3.1). From each plot one randomly positioned sample was taken. Each sample measured $16 \mathrm{x}$ $16 \mathrm{~cm}^{2}$ taken to a depth of $5 \mathrm{~cm}$ of the mineral soil. Litter and soil layers were separated in the field. Then, samples were transported to the laboratory for extraction of soil animals. Animals were extracted by heat for three days (Kempson et al., 1963), collected in dimethyleneglycol - water solution $(1: 1)$ and stored in $70 \%$ ethanol until further processing. Environmental variables were measured in composite samples of each litter and soil (five cores per plot within a radius of ca. $2 \mathrm{~m}$ around the soil animal sample), including abiotic factors ( $\mathrm{pH}$, water content, $\mathrm{C}$-to- $\mathrm{N}$ ratio) and biotic factors (microbial community composition in litter and soil as indicated by phospholipid fatty acids; Krashevska et al., 2015, V. Krashevska unpubl. data; see Appendix Tables 3.1 and 3.2). Litter and soil $\mathrm{pH}\left(\mathrm{CaCl}_{2}\right)$ was measured using a digital $\mathrm{pH}$ meter. Aliquots of litter and soil material were dried at $65{ }^{\circ} \mathrm{C}$ for $72 \mathrm{~h}$, milled and analyzed for total $\mathrm{C}$ and $\mathrm{N}$ concentrations using an elemental analyzer (Carlo Erba, Milan, Italy). Water content of litter and soil were determined gravimetrically (for more details on environmental factors see Krashevska et al., 2015). The extracted animals were sorted to high-rank taxonomic groups (mostly orders; Potapov et al., 2019). From these data we used the abundance of potential predators (Mesostigmata, Araneae and Formicidae) and potential competitors (Oribatida) of Collembola as biotic factors potentially affecting their abundance and community composition; for details see Appendix Tables 3.1. We sorted and identified all individuals of Collembola from the 2013 samples (in total 4069 individuals) and we took a random subsample of $25 \%$ of individuals from the 2016 samples (in total 986 individuals). This unequal sampling effort is expected to affect species richness, but not density and proportional community composition estimates. 


\section{Chapter 3}

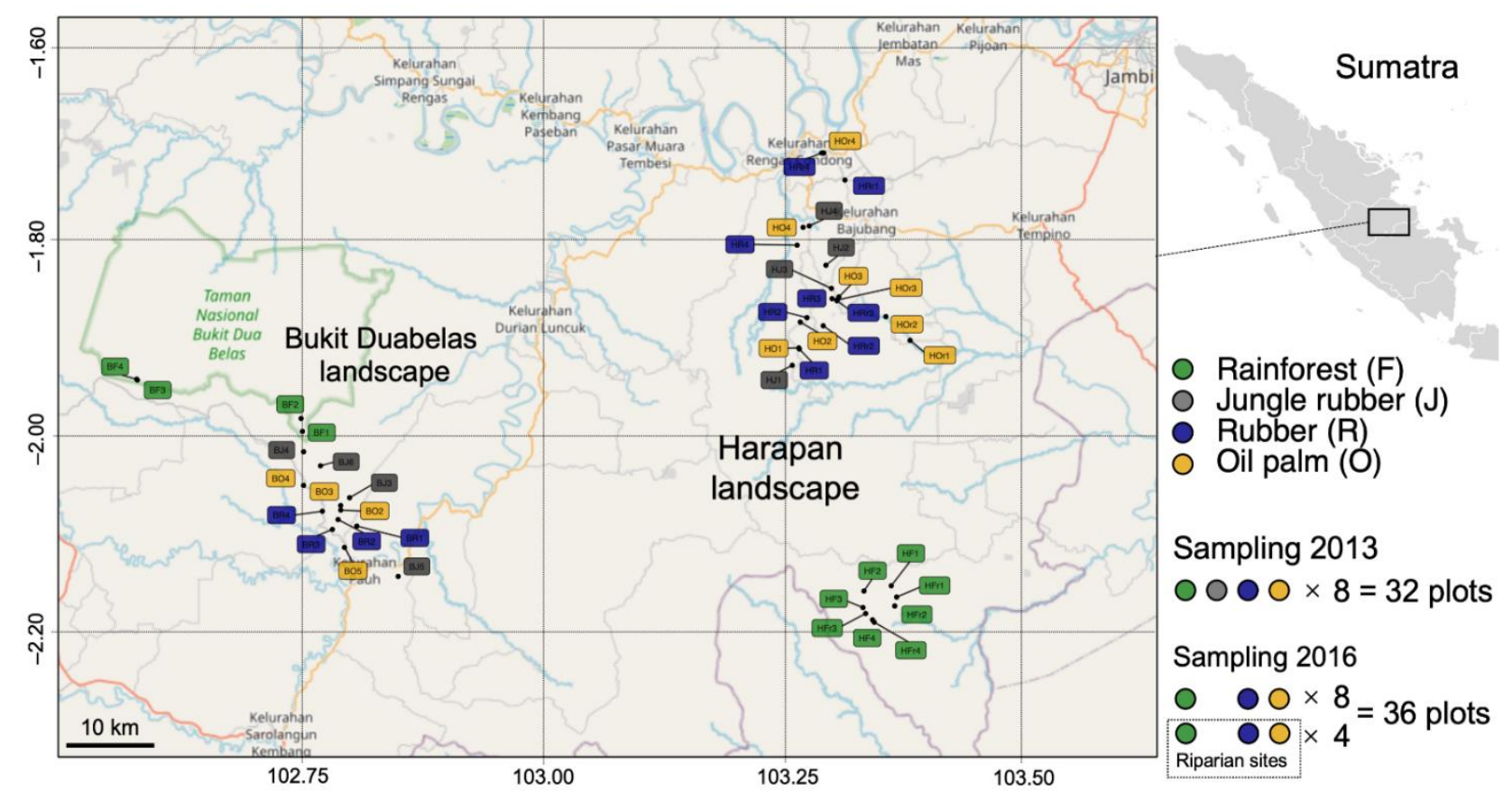

Figure 3.1. Map of the sampling locations in two landscapes (Bukit Duabelas and Harapan) in 2013 and 2016; for details see text.

\section{Species identification}

Collembola were sorted into morphogroups under a dissecting microscope (Zeiss, Stemi 508) at 50x magnification, based on basic morphological characters (body size, body shape, morphology of furca, antennae, and number of eyes). A number of individulas of each morphogroup from each sample were subsequently cleared with Nesbitt solution on a heating plate $\left(50^{\circ} \mathrm{C}\right)$ for 3-10 min. Then, the animals were mounted on slides with Hoyer's solution (for details see Glime and Wagner, 2017). Collembola were identified to species level using a compound microscope (Axiovert 35, Zeiss, Jena, Germany) at maximum 400× magnification, using the checklist for Indonesian Collembola (Suhardjono et al., 2012) and additional articles containing keys for Collembola of southeast Asia, particularly Indonesia (Potapov and Starostenko, 2002; Potapov, 2012; Mateos and Greenslade, 2015). Due to the poorly described fauna, in many cases we had to assign individuals to morphospecies without Latin binomials (in total $70 \%$ of all identified species). Below, we refer to all identifications as 'species' for simplicity. Whenever possible, juvenile specimens were ascribed to species by comparing with adults or subadults. The full list of traits and species is given in Supplementary Table S1. Along with the identification, each species was described using a set of morphological 
traits (Table 3.1). The same selected set of traits was used for each species across landuse systems for statistical analysis, including: presence/absence of empodial appendage, sucking/chewing mouthpart, presence/absence of post-antennal organ (PAO), presence/absence of scales, elongated/normal abdomen IV, elongated/spherical abdomen, separate/fused abdominal segments, presence/absence of furca, straight/curved and short/long furca, presence/absence of pigmentation, diffuse/patterned and intensive/light pigmentation, normal/modified antennae, small/medium/large body size (Table 3.1). Data on Collembola species, including their traits and pictures, were uploaded to the open virtual research environment "Ecotaxonomy" (http://ecotaxonomy.org). 
Table 3.1. Collembola traits used in the study and their potential functions. For more details on the traits see http://ecotaxonomy.org/traits. Combinations of different traits (life form) are related to trophic niches of species and thus their role in ecosystems (Potapov et al., 2016a).

\begin{tabular}{|c|c|c|c|}
\hline Trait & Potential function & Trait states & References \\
\hline $\begin{array}{l}\text { Abdominal } \\
\text { modifications }\end{array}$ & $\begin{array}{l}\text { Excretion, digestion, } \\
\text { reproduction. } \\
\text { Colonization of specific } \\
\text { (micro)habitats }\end{array}$ & $\begin{array}{l}\text { Abdomen IV } \\
\text { elongated, spherical } \\
\text { abdomen, fused } \\
\text { abdomen }\end{array}$ & $\begin{array}{l}\text { Hopkin, 1997; } \\
\text { Suhardjono et al., } \\
2012\end{array}$ \\
\hline $\begin{array}{l}\text { Antennae } \\
\text { modifications }\end{array}$ & $\begin{array}{l}\text { Sensory function, } \\
\text { modified antennae in } \\
\text { some genera (clasping } \\
\text { antennae) also used for } \\
\text { mating }\end{array}$ & $\begin{array}{l}\text { Clasping antennae, } \\
\text { subdivided antennae I } \\
\text { and II, subdivided } \\
\text { antennae III and IV, } \\
\text { antennae I very long, } \\
\text { antennae IV shorter } \\
\text { than III }\end{array}$ & $\begin{array}{l}\text { Hopkin, 1997; } \\
\text { Suhardjono et al., } \\
2012\end{array}$ \\
\hline Body size & $\begin{array}{l}\text { Metabolic demands, } \\
\text { dispersal ability, } \\
\text { predator-prey } \\
\text { interactions }\end{array}$ & $\begin{array}{l}\text { Total length from the } \\
\text { front of the head to } \\
\text { the end of the } \\
\text { abdomen: } \\
\text { small: }<0.7 \mathrm{~mm} \text {, } \\
\text { medium: } 0.7-1.2 \\
\text { mm, large: }>1.2 \mathrm{~mm}\end{array}$ & Hopkin, 1997 \\
\hline $\begin{array}{l}\text { Empodial } \\
\text { appendage }\end{array}$ & $\begin{array}{l}\text { Helping to walk, } \\
\text { particularly on wet } \\
\text { surfaces }\end{array}$ & Present, absent & Christiansen, 1965 \\
\hline $\begin{array}{l}\text { Furca } \\
\text { development }\end{array}$ & $\begin{array}{l}\text { Dispersal abilities of } \\
\text { species and predator } \\
\text { avoidance }\end{array}$ & $\begin{array}{l}\text { Furca absent, short, } \\
\text { straight furca, long } \\
\text { furca }\end{array}$ & $\begin{array}{l}\text { Hopkin, 1997; } \\
\text { Ponge and Salmon, } \\
2013\end{array}$ \\
\hline Mouthparts & $\begin{array}{l}\text { Type of food or feeding } \\
\text { strategy. Small size of } \\
\text { the apical mouth } \\
\text { opening and no molar } \\
\text { plate indicate absence of } \\
\text { capability to convey } \\
\text { solid food particles }\end{array}$ & $\begin{array}{l}\text { Molar plate present or } \\
\text { absent (piercing- } \\
\text { sucking mouthparts) }\end{array}$ & $\begin{array}{l}\text { Hopkin, 1997; } \\
\text { Suhardjono et al., } \\
\text { 2012; Adams, } 1979\end{array}$ \\
\hline $\begin{array}{l}\text { Post- } \\
\text { antennal } \\
\text { organ (PAO) }\end{array}$ & $\begin{array}{l}\text { Sensory function, } \\
\text { particularly in olfaction }\end{array}$ & $\begin{array}{l}\text { PAO absent, PAO } \\
\text { simple, PAO complex }\end{array}$ & $\begin{array}{l}\text { Hopkin, 1997; } \\
\text { Suhardjono et al., } \\
2012\end{array}$ \\
\hline Pigmentation & $\begin{array}{l}\text { UV protection, } \\
\text { thermodynamic } \\
\text { buffering and signaling, } \\
\text { camouflage }\end{array}$ & $\begin{array}{l}\text { Absent, diffuse, } \\
\text { intensive, patterned }\end{array}$ & $\begin{array}{l}\text { Hopkin, 1997; } \\
\text { Salmon et al., } 2014\end{array}$ \\
\hline Scales & $\begin{array}{l}\text { dessiccation protection, } \\
\text { thermodynamic } \\
\text { buffering and signaling, } \\
\text { potentially predation } \\
\text { avoidance }\end{array}$ & Present, absent & $\begin{array}{l}\text { Hopkin, 1997; } \\
\text { Salmon } \text { et al., 2014; } \\
\text { Hawes and } \\
\text { Greenslade, } 2015\end{array}$ \\
\hline
\end{tabular}




\section{Chapter 3}

\section{Statistical analysis}

In all analyses, we used individual soil samples as replicates, i.e. $\mathrm{n}=64$ in 2013 (32 plots x 2 layers, litter and soil) and $n=72$ in 2016 (36 plots x 2 layers, litter and soil). Area-based density and species richness were analysed as count data (individuals/species per sample). In addition, we calculated density per gram of carbon in litter and soil using the data from Krashevska et al. (2015). Statistical analyses were conducted using R version 3.5.3 (R Core Team, 2017) unless stated otherwise.

To test whether rainforest and jungle rubber have higher total density, species richness and functional diversity of Collembola than rubber and oil palm plantations (hypothesis 1), we inspected the effect of land-use system (rainforest, jungle rubber, rubber, oil palm), layer (litter, soil), land-use system - layer interaction, hydrological position (riparian, upland), year $(2013,2016)$ and landscape (Harapan, Bukit) on total density, species richness, functional diversity (FD) and functional dispersion (FDis) of Collembola. Generalized linear models with these factors were run using glmer.nb (negative binomial distribution and optimizer="bobyqa" option) in the lme4 package v. 1.1-21 (Bates et al., 2015). Soil core was included as random effect to account for interdependency of co-sampled soil and litter layers (Zuur 2009). We run several models of increasing complexity and the final model was selected using anova comparison of more complex versus simplified models based on AIC values. Significance was evaluated using the Wald chi-square test with anova in the car package v. 3.0-2 (Fox and Weisberg, 2019). Pairwise differences between treatments were assessed using Tukey contrasts by applying glth and cld in the multcomp package v. 1.4-10 (Hothorn et al., 2008). The model selection procedure is described in more detail in Appendix Tables 3.2-3.6.

To calculate FD, traits were coded as binary zero and one variables except for body length which was coded as numerical variable scaled between 0 and 1 . The trait matrix was transformed into a dendrogram using hclust and then used to calculate FD in each community (i.e., sample) using treedive in the vegan package v. 2.5-5 (Oksanen et al., 2019) (Supplementary Fig. S3.1). FDis was calculated using fdisp in the FD package and represented the weighted average distance of all species to the weighted community centroid in multidimensional space (Laliberte and Legendre, 2010). FDis is another measure of FD that, in contrast to FD, accounts for species abundances. Since the distribution of FD and FDis data were close to normal, we used lmer instead of glmer.nb. The final models were selected using the same procedure as described above. 
To test whether community composition of Collembola changes with land use (hypothesis 2), we applied linear discriminant analyses (LDA) to assess differences in community composition of Collembola species and traits among land-use systems. All species and trait values were weighted based on their relative abundance in the community. For that, we used the number of individuals of certain species or with a certain trait state. Only species occurring in at least three plots were included in the analysis (Supplementary Tables S3.2 and S3.3). Non-metric multidimensional scaling (metaMDS) with six dimensions was done before continuing with LDA. LDA was done with the MASS package using all six axes from the NMDS (Venables and Ripley, 2002). Wilks Lambda and $p$-values determined the effect of land-use system on community composition and were calculated using manova in the pander package v. 0.6.3 (Daroczi and Tsegelskyi, 2018). After this, pairwise tests between land-use systems were conducted using HotellingsT2 in the ICSNP package v. 1.1-1 (Nordhausen et al., 2018). To numerically estimate distances among communities from different land-use systems, squared Mahalanobis distances $\left(\mathrm{MD}^{2}\right)$ between land-use systems were calculated using the mahal function in the HDMD package v. 1.2 (McFerrin, 2013).

To inspect effects of environmental factors on species and traits (hypotheses 3 and 4), we used multivariate community analyses (canonical correspondence analysis (CCA) and redundancy analysis (RDA). We applied forward-selection CCA as implemented in CANOCO 5.02 (ter Braak and Smilauer, 2012) to explore the correlation between species composition of communities and environmental variables across the two sampling years. CCAs were performed because the lengths of gradients were 4.0 SD units for litter and 3.4 SD units for soil indicating unimodal species - environment relationships (Leaps, 2003). Only species occurring in at least two plots in each land-use were included in the analyses (Supplementary Tables S3.2 and S3.3). The following environmental variables were included in the CCA that were a priori assumed to affect Collembola community composition: C-to-N ratio, $\mathrm{pH}\left(\mathrm{CaCl}_{2}\right)$ value, water content $(\%)$, sum of phospholipid fatty acid (PLFA) marker lipids of Gram-positive bacteria (i15:0, a15:0, i16:0, i17:0) and

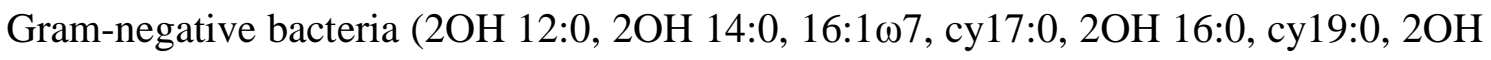

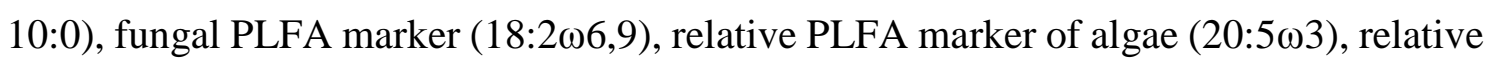
marker of arbuscular mycorrhizal fungi based on the neutral lipid fatty acid (NLFA) 16:1 $\omega 5 \mathrm{c}$, abundance of potential predators (Mesostigmata, Araneae and Formicidae), and abundance of potential competitors (Oribatida) (Appendix Tables 3.1). Monte-Carlo tests (999 permutations) were performsed to evaluate the overall model significance and the 
significance of environmental variables and individual axes. Since the global test with all environmental variables was significant, we used forward selection to identify the most important environmental variables affecting Collembola communities. The forward selection procedure was stopped if a variable reached a level of significance $>0.05$. We additionally tested for spatial autocorrelation in community data by relating pcnm spatial vectors (vegan package in $\mathrm{R}$ ) to species matrix using CCA (cca function in $\mathrm{R}$ ). We separately analysed data from 2013 and 2016 after pooling soil and litter data from the same soil cores. Although some spatial patterns were observed visually, the results of anova showed no significant spatial effects $\left(p=0.143\right.$, adjusted $\mathrm{R}^{2}=0.03$ in 2013 and $p$ $=0.141$, adjusted $\mathrm{R}^{2}=0.03$ in 2016).

Correlations between trait composition of communities (Supplementary Table S3.4 and S3.5) and environmental variables was analysed using RDA as implemented in CANOCO 5.02 (ter Braak and Smilauer, 2012). RDA instead of CCA was performed because gradients of the trait data were 2.5 SD units for litter and 2.0 SD units for soil indicating linear trait-environment relationships (Leaps, 2003). Hellinger standardization, recommended for RDA, was applied to the data prior to the analysis. The same twelve environmental variables as in CCA (see above) were included in the RDA analysis and the same procedures were used to assess significance of the environmental factors included. Land-use systems, regions and years were included as silent variables not affecting the ordination, and displayed on both CCA and RDA figures.

\section{Results}

\section{Density, species richness, functional diversity (FD) and functional dispersion (FDis)}

In total 5055 individuals were assigned to 54 species from 27 genera and 13 families across all land-use systems and two sampling years with $22.2 \%$ of total species found in all land-use systems, $11.1 \%$ only in rainforest, $3.7 \%$ only in jungle rubber, 3.7 $\%$ only in rubber plantations, and $11.1 \%$ only in oil palm plantations, while remaining $\sim 48 \%$ were found in two or three land-use systems (Fig. 3.2). Rarefaction curves of the samples indicated that the number of species saturated in jungle rubber and rubber plantations in 2013 and all systems in 2016, and were close to saturation in rainforest and oil palm plantations in 2013 (Supplementary Material Fig 3.1). The number of Collembola per unit area was $66 \%$ higher in litter than in soil, but did not significantly 
differ among land-use systems (Fig. 3.3a, Table 3.2). Across the two sampling years, differences in density and species richness among land-use systems were moderate, and the main effect was the land-use system $\times$ layer interaction due to significantly lower density $(-50 \%)$ and species richness (-30\%) of Collembola in litter than in soil of oil palm plantations than in rainforest (Fig. 3.3b, c, Table 3.2). Although post-hoc pairwise comparions did not show significant differences, both density and species richness of Collembola in the litter layer tend to be higher in jungle rubber and rainforest than in rubber and oil palm plantations (-25 to $-40 \%$ ) (Fig. 3.3b, c). Density and species richness of Collembola in the soil layer also did not differ significantly among land-use systems, being even slightly higher in oil palm plantations than in the other land-use systems. FD in the litter layer was higher (23-26\%) in litter of rainforest than in litter of plantations. By contrast, FD in the soil layer did not differ significantly among the land-use systems (Fig. 3.3d, Table 3.2). Both species richness and FD were strongly affected by year presumably due to different sampling effort in the two years (see Methods). FDis did not differ significantly among land-use systems according to post-hoc comparisons, but it was generally highest in the litter layer in rainforest and lowest in the litter layer in oil palm plantations (significant land-use system $\times$ layer interaction; Table 3.2).

Table 3.2. Wald chi-square test on the effect of layer, land-use system, year, riparian, landscape and their interaction on characteristics of Collembola communities based on mixed-effects models. Factors that were not selected by the model based on AIC are denoted by dashes.

\begin{tabular}{|c|c|c|c|c|c|}
\hline Factor & D.f. & $\begin{array}{c}\text { Density } \\
\left(\text { ind. } \mathrm{m}^{-2}\right)\end{array}$ & $\begin{array}{c}\text { Species } \\
\text { richness } \\
\text { (per sample) }\end{array}$ & $\begin{array}{l}\text { Functional } \\
\text { diversity } \\
\text { (FD per } \\
\text { sample) }\end{array}$ & $\begin{array}{c}\text { Functional } \\
\text { dispersion } \\
\text { (FDis per } \\
\text { sample) }\end{array}$ \\
\hline Layer & 1 & 2.7 & 1.4 & 2.9 & 0.1 \\
\hline Land-use system & 3 & 6.7 & 7.5 & 6.4 & 1.2 \\
\hline Year & 1 & - & $48.7 * * *$ & $29.4 * * *$ & 3.8 \\
\hline Riparian & 1 & - & - & 0.1 & - \\
\hline Landscape & 1 & 3.5 & - & 3.6 & - \\
\hline $\begin{array}{l}\text { Layer } \times \text { Land-use } \\
\text { system }\end{array}$ & 3 & $11.8 *$ & $14.5 * *$ & $14.0 * *$ & $12.7 * *$ \\
\hline
\end{tabular}


Chapter 3

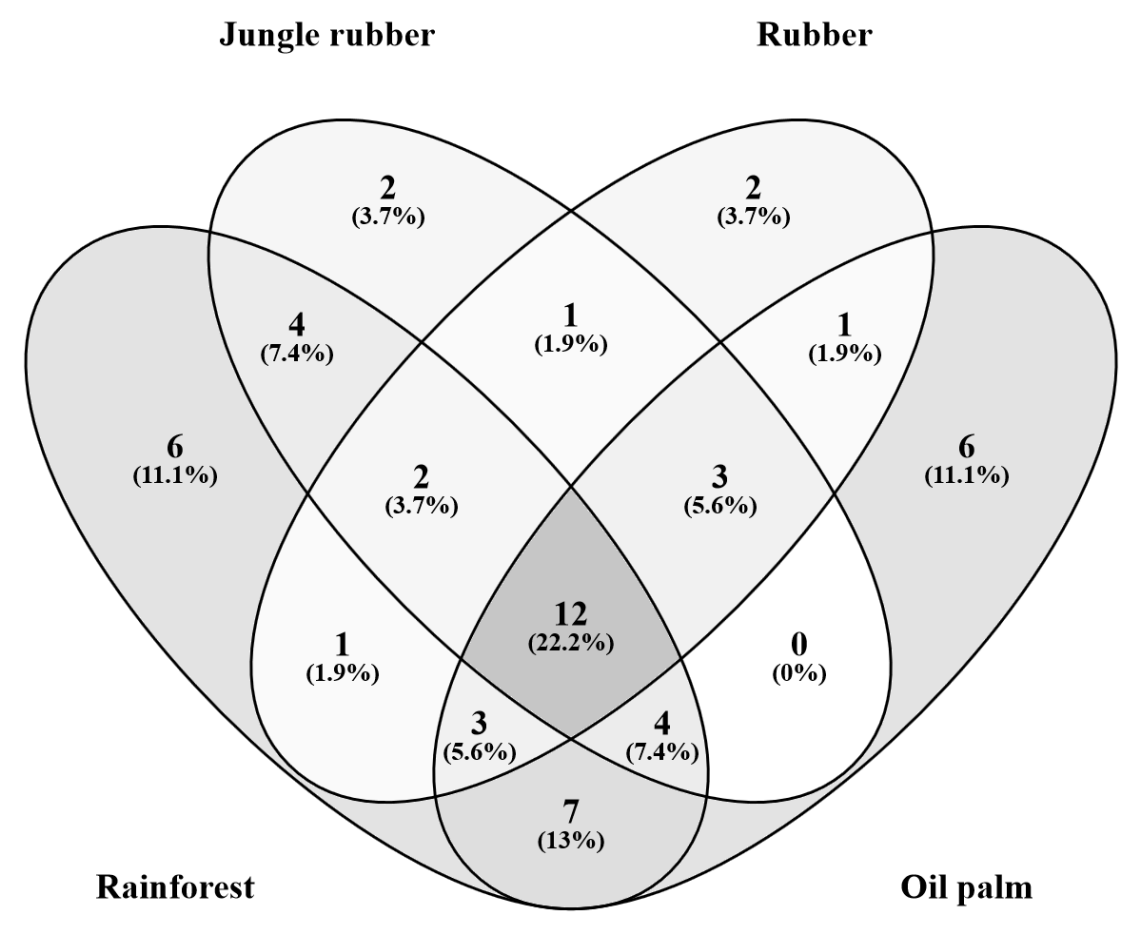

Figure 3.2. Venn diagram of species in different land-use systems (rainforest, jungle rubber, rubber and oil palm plantations). 


\section{Chapter 3}
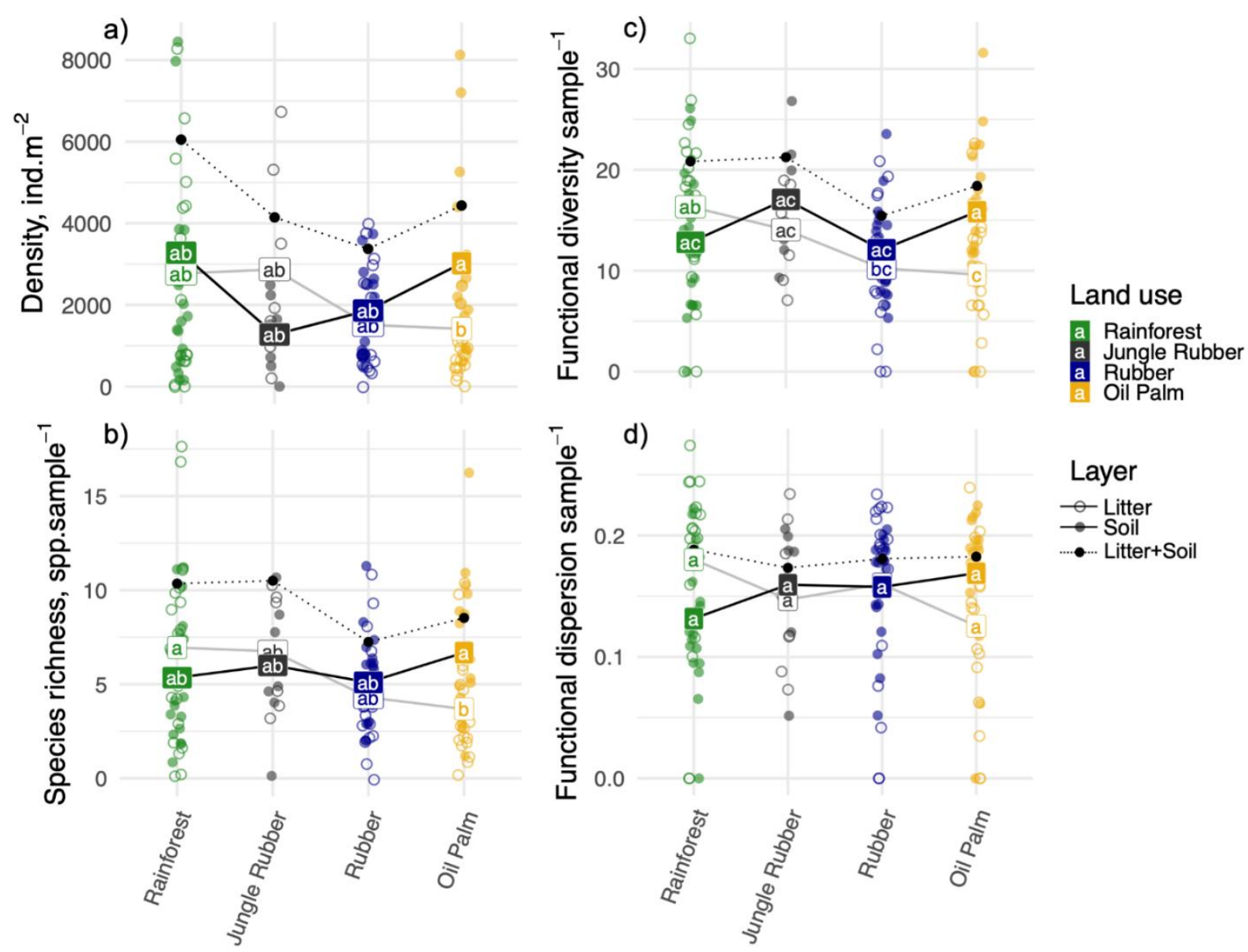

Figure 3.3. Density and species richness of Collembola in different land-use systems across sampling years. (a) Density of Collembola per square meter, (b) number of Collembola species per sample $\left(256 \mathrm{~cm}^{2}\right)$, (c) functional diversity (FD) of Collembola communities per sample, and (d) functional dispersion (FDis) of Collembola communities per sample. Each soil core was divided into litter and soil layers $(0-5 \mathrm{~cm})$, and these layers were treated as replicates and presented as separate points in the figure (open points - litter, filled points - soil). Labels connected by solid lines show mean values for litter and soil separately (white labels - litter, black labels - soil). Mean values across layers and systems sharing the same letter are not significantly different for the given variable (Tukey contrasts). Black points connected with dotted lines show mean values for litter and soil combined (sum of density, newly calculated after combining layers for species richness, FD and FDis).

\section{Species and trait composition of communities}

Of the 54 species identified, Folsomides centralis, Isotomiella spp. and Pseudosinella sp. 1 were most abundant across land-use systems (Appendix, Figs 3.2). Land use had a pronounced effect on the species composition of Collembola communities in the litter layer, with the first LDA axis explaining $71 \%$ of the variation (Wilks' lambda 
$=0.32$, approx. $\mathrm{F}=8.89, p<0.001$; Fig. 3.4a). Separation of communities by different land-use systems was also evident if the years 2013 (Wilks' lambda $=0.11$, approx. $\mathrm{F}=$ 9.47, $p<0.001$ ) and 2016 (Wilks' lambda $=0.23$, approx. $\mathrm{F}=14.64, p<0.001$ ) were analyzed separately (Appendix, Figs 3.3,4). Species composition of Collembola communities was different in each of the land-use systems with rainforest being more similar to oil palm than to jungle rubber. Collembola communities in jungle rubber were similar to those in rubber plantations, while rubber and oil palm plantations were distinct. Differences between land-use systems were associated with changes in species dominance: Isotomiella spp. and Pseudosinella sp.1 were the dominant species in rainforest, Ascocyrtus cinctus was dominant in rubber plantations, and Rambutsinella cf. scopae was dominant in oil palm plantations (Appendix, Figs 3.2).

Land use also significantly affected the species composition of Collembola communities in the soil layer, with the first LDA axis explaining $48 \%$ of the variation (Wilks' lambda $=0.48$, approx. $\mathrm{F}=5.64, p<0.001$; Fig. 3.4c) which was evident in both years (Wilks' lambda $=0.23$, approx. $\mathrm{F}=5.37, p<0.001$ for 2013 and Wilks' lambda $=$ 0.49 , approx. $\mathrm{F}=4.38, p<0.001$ for 2016) (Appendix, Figs 3.3,4). Isotomiella spp. reached very high abundance in rainforest and was also abundant in oil palm plantations, but was less abundant in jungle rubber and rubber plantations. Pseudosinella sp.1 reached high abundance across all land-use systems except for jungle rubber, and Folsomides centralis reached high abundance in oil palm plantations (Appendix, Figs 3.2).

Similar to species composition, land use also strongly affected the trait composition of Collembola communities in the litter layer, with the first LDA axis explaining 53\% of the variation (Wilks' lambda $=0.39$, approx. $\mathrm{F}=7.22, p<0.001$; Fig. $3.4 b$ ), which was evident in 2013 (Wilks' lambda $=0.17$, approx. $\mathrm{F}=8.62, p<0.001$ ) and 2016 (Wilks' lambda $=0.36$, approx. $\mathrm{F}=9.14, p<0.001$ ) (Appendix, Figs 3.3,4). In contrast to species community composition, trait community composition in rainforest was most distinct between rainforest and oil palm plantations, and intermediate in jungle rubber and rubber plantations. Absence of empodial appendage, sucking mouthparts, absence of furca, small body size, absence of pigmentation and spherical abdomen were abundant traits in rainforest and jungle rubber. By contrast, long furca, present of scales, large size, patterned and diffuse pigmentation, straight furca and presence of PAO were abundant traits in plantation systems. Details of Mahalanobis distances for species and trait community composition in the litter layer are given in Appendix Tables 3.7 and 3.8. 


\section{Chapter 3}

Also, trait composition of Collembola communities in the soil layer was significantly affected by land use with the first LDA axis explaining $57 \%$ of the variation (Wilks' lambda $=0.45$, approx. $\mathrm{F}=6.18, p<0.001$; Fig. 3.4d), which was evident in both years (Wilks' lambda $=0.23$, approx. $\mathrm{F}=5.30, p<0.001$ for 2013 and Wilks' lambda $=$ 0.45 , approx. $\mathrm{F}=7.29, p<0.001$ for 2016) (Appendix, Figs 3.3,4). Trait community composition in rubber plantations was similar to that in oil palm plantations in both years (characterized by diffuse pigmentation, straight furca, presence of PAO, modified antennae, large size and patterned pigmentation). By contrast, absent or intense pigmentation, absence of empodial appendage, small size and spherical abdomen were abundant traits in rainforest. Details of Mahalanobis distances for species and trait community composition in the soil layer are given in Appendix, Tables 3.7 and 3.8.
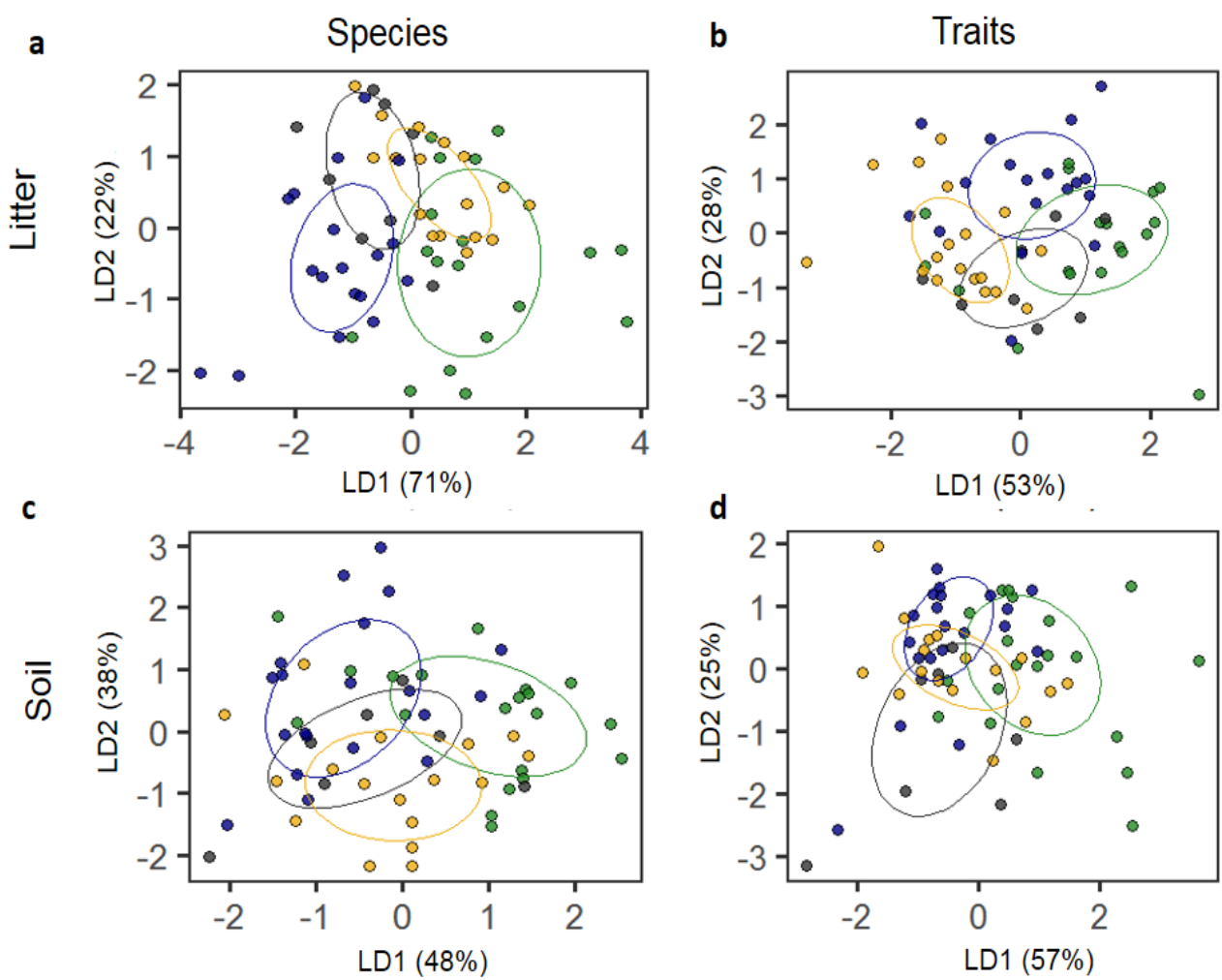

\section{Land use}

- Rainforest

- Jungle rubber

- Rubber

- Oil palm

Figure 3.4. Linear discriminant analysis (LDA) of species (a, c) and trait composition (b, d) of Collembola communities in litter (a, b) and soil (c, d); data pooled for sampling years (2013 and 2016). Land-use system was used as grouping variable. Ellipses were calculated using the MASS package in $R$ to visualize the grouping of species or traits in the different land-use systems. Each point represents a sample. 


\section{Chapter 3}

\section{Correlation between environmental factors and community composition}

In the litter layer, four of the twelve environmental variables significantly correlated with species composition (CCA, forward selection), explaining $13.6 \%$ of the variation in species composition (Trace $=0.83, \mathrm{~F}=1.59, p=0.001$; Fig. 3.5a). Litter $\mathrm{pH}$ accounted for $4.2 \%$ of total variation in species composition (pseudo $\mathrm{F}=2.5, p=0.002$ ), water content for additional $3.9 \%$ (pseudo $\mathrm{F}=2.4, p=0.003$ ), density of Mesostigmata for $2.8 \%$ (pseudo $\mathrm{F}=1.7, p=0.033$ ) and relative amount of Gram-negative bacterial PLFAs for $2.8 \%$ (pseudo $\mathrm{F}=1.8, p=0.025$ ). In 2013 rainforest and jungle rubber communities were associated with high water content and high density of Mesostigmata, whereas in 2016 rainforest communities were associated with high amount of Gramnegative bacteria only. Communities in rubber and oil palm plantations both in 2013 and 2016 were associated with litter pH. Several atmobiotic and epedaphic species, i.e., Acrocyrtus sp.3, Lepidocyrtus sp.1, Pararrhopalites sp.1, Sphaeridia sp.1 and Sphaeridia sp.2, but also euedaphic species, i.e., Pseudosinella sp.1, Isotomiella spp. and Onychiuridae spp., were associated with high water content and density of Mesostigmata, whereas Superodontella sp.1, Xenylla sp.1 and Callyntrura sp.1 were associated with Gram-negative bacteria. Ascocyrtus cinctus, Rambutsinella cf. scopae, Folsomides parvulus, Folsomides centralis, Homidia cingula, Acrocyrtus sp.1, and Megalothorax cf. minimus in plantation systems were associated with high litter $\mathrm{pH}$.

In the soil layer, only two of the twelve environmental variables significantly correlated with species composition, together explaining $5.7 \%$ of the variation (Trace = $0.67, \mathrm{~F}=1.27, p=0.02$; Fig. 3.5b). Based on forward selection, density of Araneae accounted for $2.9 \%$ of the total variation in community composition (pseudo $\mathrm{F}=1.7, p$ $=0.044$ ) and soil $\mathrm{pH}$ accounted for additional $2.8 \%$ (pseudo $\mathrm{F}=1.7, p=0.045$ ). In line with the effects of environmental factors in the litter layer, in the soil layer high $\mathrm{pH}$ was associated with high density of several atmobiotic species, i.e., Homidia cingula, Isotomurus cf. parabalteatus, Dicranocentrus sp.1 and Acrocyrtus sp.1. By contrast, Pseudosinella sp.1, Isotomiella spp., Sphyrotheca sp.1, Cyphoderopsis sp.1, Xenylla sp.1 and Folsomides parvulus were associated with high density of Araneae (potential macropredator) in rainforest. 
$\mathbf{a}$

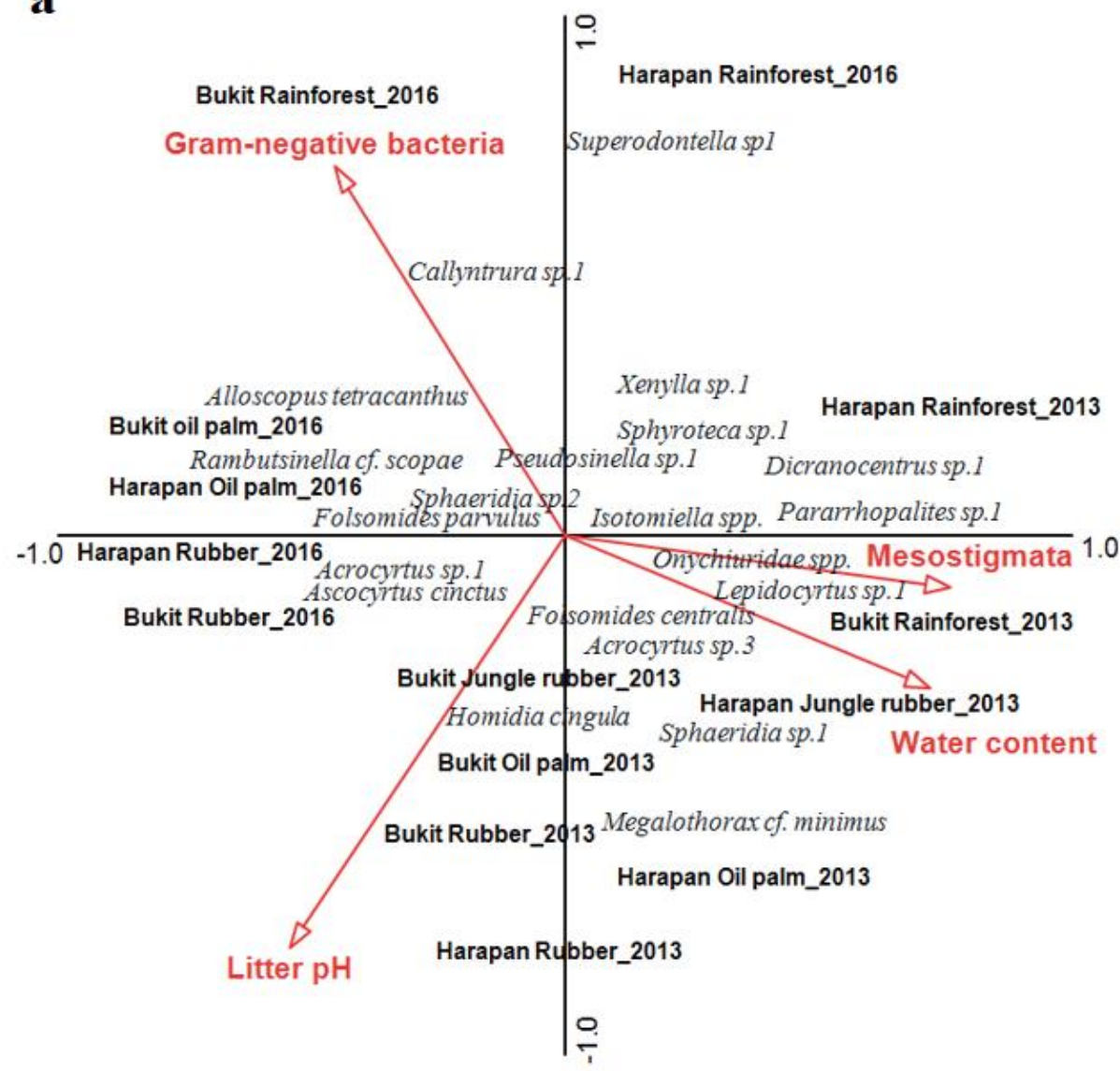

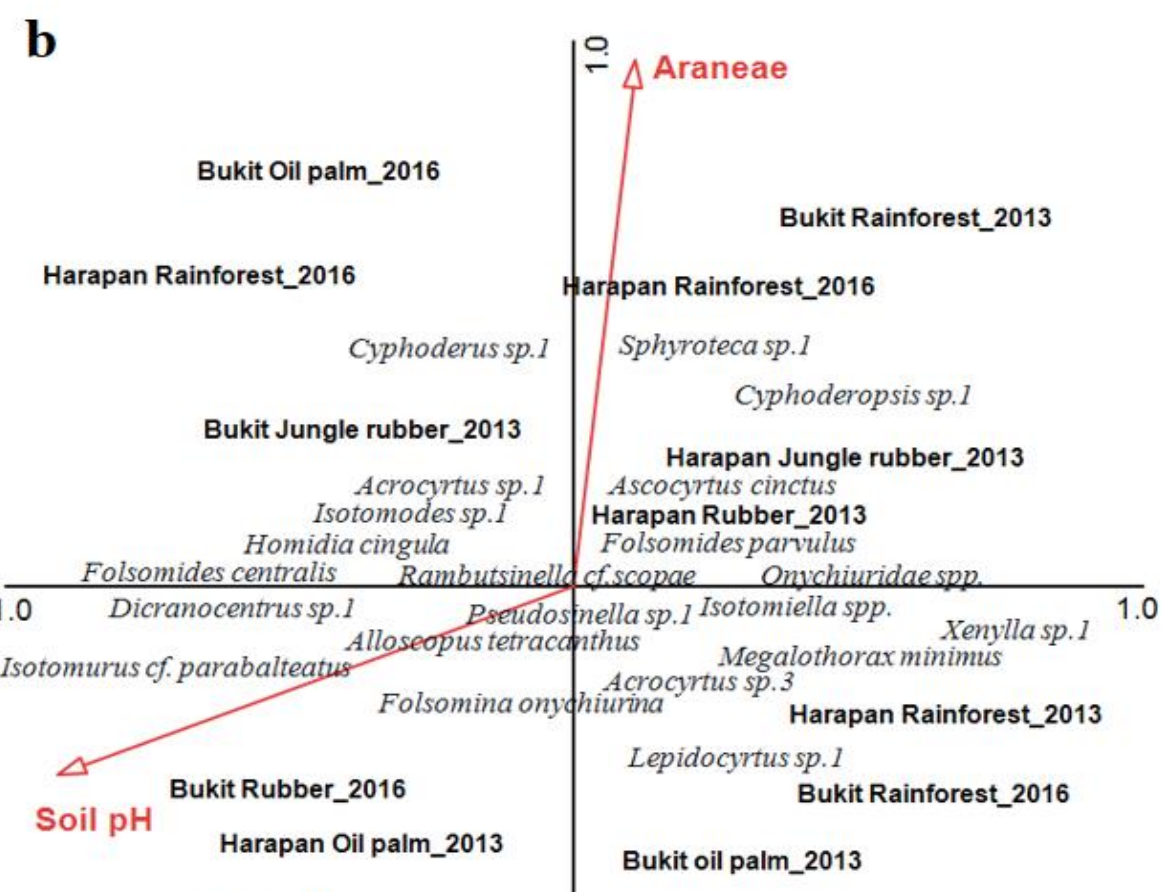

Harapan Oil plm_2016

Figure 3.5. Canonical correspondence analysis of Collembola species in (a) litter and (b) soil as related to environmental variables (red arrows). Only variables selected as being significant in the forward selection procedure are shown. The four land-use systems (rainforest, jungle rubber, rubber plantations, oil palm plantations), two years $(2013,2016)$ and both regions (Harapan, Bukit Duabelas) are shown together. The name of species and land-use systems were centred to their position. The length of arrows represent the percentage variation explained by environmental variables. For environmental data see Appendix, Tables 1 and 2. 
In the litter layer, none of the environmental variables were correlated significantly with the trait composition of Collembola communities (RDA, forward selection; Trace = $0.22, \mathrm{~F}=1.09, p=0.27$; Fig. 3.6a). While the overall model was not significant, both water content and $\mathrm{pH}$ were identified as significant factors, accounting for 3.6 and $3.3 \%$ of the total variation in trait composition, respectively (pseudo $\mathrm{F}=2.2, p=0.045$ and $\mathrm{F}=2.1, p=$ 0.048 , respectively).

In the soil layer, four of the twelve environmental variables were significant, explaining $15.2 \%$ of the variation in the trait composition (Trace $=0.26, \mathrm{~F}=1.39, p=0.02$; Fig. 3.6b). Based on forward selection, water content accounted for $4.4 \%$ of total variation in trait composition (pseudo $\mathrm{F}=2.7, p=0.022$ ), soil $\mathrm{pH}$ for $3.9 \%$ (pseudo $\mathrm{F}=2.5, p=0.012$ ), abundance of Mesostigmata for 3.4\% (pseudo $\mathrm{F}=2.2, p=0.026$ ) and the fungal PLFA marker $(18: 2 \omega 6,9)$ for $3.4 \%$ (pseudo $\mathrm{F}=2.2, p=0.014$ ). High $\mathrm{pH}$ values were correlated positively with diffuse pigmentation, straight furca, presence of PAO, presence of scales, modified antennae, large body size and patterned pigmentation. High water content and to some extent the fungal PLFA marker were correlated positively with absence of furca, small body size, spherical and fused abdomen, absent and intensive pigmentation, and sucking mouthparts. In addition, high density of Mesostigmata were correlated positively with long furca and absence of empodial appendage. 

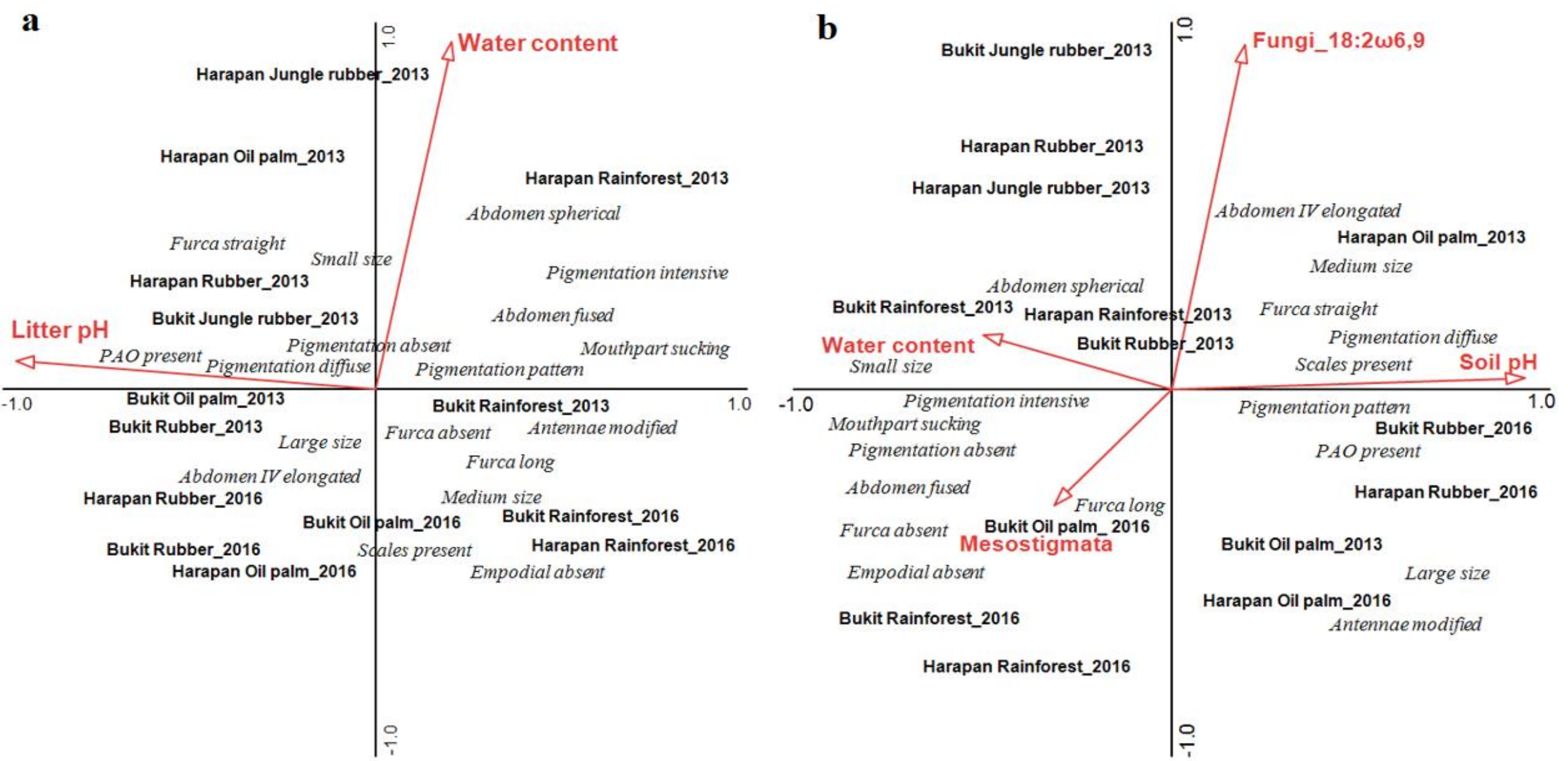

Figure 3.6. Redundancy analysis of Collembola traits in (a) litter and (b) soil as related to environmental variables (red arrows). Only variables selected as being significant during the forward selection procedure are shown. All land-use systems (rainforest, jungle rubber, rubber plantations, oil palm plantations), two years $(2013,2016)$ and both regions (Harapan, Bukit Duabelas) are shown together. The traits and land-use systems were centred to their position. The length of arrows represent the percentage variation explained by environmental variables. For environmental data see Appendix, Tables 1 and 2. 


\section{Chapter 3}

\section{Discussion}

Our study comprehensively assessed the response of the species and trait composition of soil and litter Collembola communities after conversion of rainforest into plantation systems. The results indicate that density, species richness and functional diversity indices of Collembola vary moderately among the studied land-use systems compared to other animal groups, which responded more strongly to the conversion of rainforest into agricultural plantation systems (Deharveng, 1996; Rusek, 1998; Sousa et al., 2006; Devi et al., 2011; Clough et al., 2016). However, density, species richness and functional diversity (FD) consistently declined in the litter layer of rubber and especially oil palm plantations. Despite the only moderate changes in community metrics, trait and species composition of Collembola communities were distinct in all land-use systems studied. Even in the extensively managed jungle rubber agroforesty system, species and trait composition of Collembola communities differed clearly from that in rainforest. Among the studied environmental variables, Collembola community composition was related closest to $\mathrm{pH}$ and water content with correlations between environmental factors and changes in communities being more pronounced in litter than in soil.

\section{Density, species richness, functional diversity and functional dispersion}

In part supporting our first hypothesis, density, species richness and FD of Collembola were at a maximum in litter of rainforest. This is in line with the study of Martius et al. (2004) in central Amazonia reporting more Collembola species in rainforest than in rubber and peach palm plantations. In another study from Jambi province, density and species richness of soil Collembola decreased gradually from rainforest to jungle rubber to rubber plantations (Deharveng, 1992 in Michon and de Foresta, 1995). This was expected since there is less litter (Krashevska et al., 2015), and thus less space and food for Collembola in rubber and even less in oil palm plantations. The amount of litter is likely to be the main factor driving Collembola communties since, according to our results, the density of Collembola per gram of carbon in the litter was similar across all land-use systems. In trend, species richness, FD and FDis also were lowest in the litter layer of oil palm plantations. This suggests that in intensively managed plantations mulching practices may help in increasing the density and species richness of Collembola, and ecosystem processes Collembola are involved in (Tao et al., 2018). 
In contrast to our first hypothesis, density, species richness and diversity indices of Collembola were similar or even slightly higher in soil of oil palm plantations than in soil of rainforest. This suggests that the soil in plantations provides suitable environmental conditions for Collembola allowing a variety of species to survive and successfully reproduce even in monoculture plantations of rubber and oil palm. The land-use effect was least pronounced for the total community FDis, suggesting that species with different functional traits may reach high densities in monoculture plantations. The results are in contrast to the study of Winck et al. (2017) in southern Brazil, which found that functional diversity of Collembola was significantly higher in tropical forest than in grassland and plantation systems. High density of soil Collembola in plantations may be related to the soil buffering Collembola species against adverse environmental conditions, thereby allowing them to cope with disturbances (Filser, 1995). For the temperate zone it has been shown that Collembola may reach high population densities even in intensively managed agroecosystems (Filser, 1995; Frampton and Brink, 2002; Chauvat et al., 2007). In Borneo the conversion of rainforest into oil palm plantations reduced species richness in a large number of taxa except in scavenging mammals and Collembola (Edwards et al., 2014). In general, soil food webs in oil palm plantations are not limited by the available energy in comparison to rainforest (Potapov et al., 2019). Fertilization and weeding likely provide additional resources for the detrital system in plantations (Clough et al., 2016). Further, Susanti et al. (2019) reported that algae serve as important food resource for Collembola in tropical ecosystems, especially in oil palm plantations. Schulz et al. (2019) reported the density of photoautotrophic protists to be strongly increased in oil palm plantations compared to rainforest at our study sites. In addition, it is increasingly recognized that Collembola benefit from root-based resources in both forests and agricultural systems (Fujii et al., 2014; Potapov et al., 2016; Li et al., 2020) and this likely contributed to the high density and number of species of Collembola in plantations. Another explanation for the high density of Collembola in soil of oil palm plantations may be reduced top-down control by predators. In fact, the density of small soil-dwelling spiders, one of the main Collembola predators (Lawrence and Wise, 2000), is low in plantations (Potapov et al., 2020).

\section{Species and trait community composition}

Supporting in part our second hyphothesis, communities of Collembola differed among all the four land-use systems studied. However, in contrast to our expectations 
community composition in jungle rubber and rainforest was distinctly different, especially in the litter layer. Further, unexpectedly, a number of Collembola species present in rainforest were also present in monoculture plantations. The community core included Isotomiella spp. and Pseudosinella sp.1 in rainforest, which were replaced by Folsomides spp. in jungle rubber and monoculture plantations, suggesting a uniform change in community composition already starting in extensively managed agroforestry systems, i.e. jungle rubber. Folsomides spp. are pantropical parthenogenetic species, usually numerically dominant in soils of disturbed ecosystems all over the tropics. Our study shows that they may reach high density in disturbed habitats even if the disturbance level is low. The observed shift in community composition is also in line with strong changes in energy channeling in the belowground food web after conversion of rainforest into agricultural landuse systems at our study sites, i.e. increased energy sequestration in large decomposers and decreased predation (Potapov et al., 2019). This higlights the susceptibility of the belowground system to changes in land use.

Confirming our third hypothesis, water content and $\mathrm{pH}$ were the most important environmental factors explaining species and trait composition of Collembola in both litter and soil. Generally, effects of environmental factors were more pronounced in litter than in soil, presumably because soil buffers variations in environmental conditions (Cassagnau 1961), whereas biota in the litter layer are more heavily exposed to such variations (Krashevska et al., 2015, 2019). The strong effect of $\mathrm{pH}$ in structuring the species and trait composition of Collembola likely is related to the increase in soil $\mathrm{pH}$ with the conversion of rainforest into plantation systems and associated changes in microbial community composition (Brinkmann et al., 2019; Berkelmmann et al., 2020). The functional link between $\mathrm{pH}$ and changes in trait composition, however, remains to be discovered. Moreover, most of the variation in Collembola community composition in litter and soil remained unexplained suggesting that stochastic or spatial processes were of major importance.

Species composition in litter in rainforest was associated with high water content, and high density of Mesostigmata and Gram-negative bacteria. The relevance of water content for Collembola community composition is in line with a number of studies from temperate regions (Ponge 1993; Kuznetsova 2003; Fujii et al., 2014). Soil moisture controls the risk of desiccation and the availability of microorganisms as food for Collembola (Fierer et al., 2003; Rousk et al., 2010; Zhang et al., 2013). High amounts of organic matter and high water holding capacity in rainforest soil likely improves habitat conditions and increases the availability of food resources in particular for eudaphic Collembola (Muturi et 
al., 2011). The presence of a number of species, including Acrocyrtus sp.3, Lepidocyrtus sp.1, Pararrhopalites sp.1, Sphaeridia sp.1, Sphaeridia sp.2 and two euedaphic species (Pseudosinella sp.1 and Onychiuridae spp.) in rainforest, as well as in jungle rubber, were associated with high water content. Further, the density of some Collembola species in litter of rainforest also correlated with the amount of Gram-negative bacteria, especially two Poduromorpha species, i.e. Xenylla sp.1 and Superodontella sp.1, suggesting that these species may feed on bacteria or bacterial feeding nematodes. Species in litter and soil of plantations, such as Homidia cingula, Isotomurus cf. parabalteatus, Dicranocentrus sp.1, Acrocyrtus sp.1, Folsomides centralis and Megalothorax cf. minimus, were associated with high $\mathrm{pH}$. In litter, however, high $\mathrm{pH}$ also was associated with low concentrations of Gramnegative bacteria.

Salmon et al. (2014) showed that traits of species living in open habitats and adapted to light exposure and dry conditions included high mobility (long furca), large body size, presence of scales, pigmentation (as protection against UV) and organs for sensing wind and light. Further, Winck et al. (2017) reported that open habitat is linked to sensorial traits (number of ocelli, antenna length and trichobothria) and drought tolerance traits (body size). Salmon and Ponge (2012) also showed that large mobile species were associated with agricultural systems with low amounts of litter. All these traits of large mobile species were also associated with plantations in our study. Presence of more Collembola with patterned coloration in plantations in comparison to rainforest was in line with more pigmented ground spiders at these sites (Potapov et al., 2020). Presumably, coloration patterns in grounddwelling arthropods (epedaphic Collembola and spiders) function as camouflage in rubber and oil palm plantations with more open canopies and shallow litter layer. Recording such universal trait patterns across groups may help linking traits and environmental conditions in the future. However, more data are needed to achieve this goal. For example, in our study Collembola species with PAO reached high density in plantations, whereas the density of Collembola species with PAO was found to be low in agricultural sites in the temperate region (Salmon and Ponge, 2012; Salmon et al., 2014). In our study, Collembola traits correlated with water content and soil $\mathrm{pH}$, whereas this was not the case in the study of Salmon and Ponge (2012), suggesting that trait - environment relationships may differ between temperate and tropical ecosystems or between different types of soils.

Finally, in contrast to our fourth hypothesis, species composition varied in a more predictable way with environmental factors than trait composition, particularly in the litter layer, where environmental factors did not significantly influence the trait composition of 
Collembola. Due to the scarcity of information on the biology of tropical Collembola, we focused on morphological traits, which we assumed to be functional (Vandewalle et al., 2010). More detailed information on functional traits, including biological, physiological and morphological traits, is needed for understanding trait - environment relationships in Collembola (Raymond-Léonard et al., 2019). On the other hand, the environmental factors included in this study may have missed important drivers of Collembola community composition. For example, the community composition of Collembola in rubber plantations differed from that of the other studied land-use systems, but the environmental factors studied failed to explain this difference. Hence, while supporting the usefulness of the traitbased approach, our analysis also highlighted the need for more information on functional traits of Collembola in tropical regions.

\section{Dominant tropical Collembola}

Dominating species at our study sites were previously recorded across various tropical regions, suggesting that certain Collembola species/taxa are well adapted to and widespread across tropical ecosystems. Collembola communities were dominated by species of the family Isotomidae, particularly the genera Folsomides and Isotomiella (43\% of total individuals). This is in line with the study of Warino et al. (2017) in Jambi province who reported that Folsomides and Isotomiella may represent $40 \%$ of total Collembola density in plantation systems. In tropical montane forests of the Doi Inthanon in Thailand Deharveng et al. (1989) also found Isotomiella spp. among the dominant species, in particular in organic layers. Similarly, Muturi et al. (2011) reported Isotomiella species to be among the most abundant Collembola in intensively managed agricultural systems in Kenya. Several species of this genus with similar morphology have been found in Sumatra (Deharveng and Suhardjono, 1994), but their ecology is little known. Three of them are particularly abundant in Sumatra (I. symetrimucronata Najt and Thibaud, 1987, the most abundant, I. nummulifer Deharveng and Oliveira, 1990, and I. cribrata Deharveng and Suhardjono, 1994). Further information on Isotomiella species in different land-use systems is needed to allow insight into the pantropical diversification of this genus.

The other dominant genus of Collembola at our study sites was Folsomides, with the species $F$. centralis and $F$. parvulus. These two species are also co-dominant in many tropical regions accross the world, with the latter especially abundant in disturbed habitats (Deharveng et al., 2020). At our study sites, F. centralis dominated in each of the plantations, 
confirming its preference for disturbed habitats. Similarly, in the tropical region of Oaxaca (Mexico) Rojas et al. (2009) reported Folsomina onychiurina to dominate in disturbed systems. At our study sites, $F$. onychiurina was also present but it was rare. An important characteristic of these pantropical Isotomidae is their mode of reprocuction: all species stated above are parthenogenetic, which may explain their large distribution across the world, as well as their capacity to colonize disturbed habitats. Besides Isotomidae, species of the family Entomobryidae reached high density in soil in most land-use systems. Pseudosinella sp.1 reached high abundance (30\%), reflecting that this species also is well adapted to the environmental conditions in agro-ecosystems. This is in line with the study of Rojas et al. (2009) reporting that Pseudosinella sp.1 may reach high density in fallow soils. The records of another Entomobryidae species, Ascocyrtus cinctus, from our study sites also fits earlier studies from Sumatra (Fatimah et al., 2012, Selvany, 2018).

Collembola communities across contrasting land-use systems were numerically dominated by small, white or pale-coloured species, often lacking eyes. However, true euedaphic (i.e., soil-dwelling) species of the families Onychiuridae and Tullbergiidae, typically abundant in temperate ecosystems, were rare in our study region, presumably due to biogeographical history rather than current ecological conditions (Deharveng et al., 1989). The similarity of Collembola communities across tropical regions suggests that several abundant pantropical species dominate in many natural and transformed tropical ecosystems, either due to historical reasons or adaptation to high temperature and other climate characteristics. Different preferences observed in pantropical genera and species of Collembola call for more focused studies on their genetic diversity and ecological preferences across tropical regions to reveal the history and diversification of Collembola across the tropics, and to understand their contribution to soil community functionning.

\section{Conclusions}

Overall, changes in density, species richness and diversity indices of Collembola communities with conversion of rainforest into plantation systems were moderate, and were more pronounced in the litter than the soil layer. Collembola density and species richness were higher in litter than in soil in rainforest, whereas in oil palm plantations it was the opposite. Community composition of Collembola changed strongly with rainforest conversion, but certain pantropical species were present in high numbers across the studied land-use systems, suggesting that these species are resistant to rainforest conversion and 
associated changes in environmental factors. Unexpectedly, the community composition of Collembola differed to a similar extent between rainforest, jungle rubber and monoculture plantations suggesting that Collembola communities are sensitive to even moderate changes in land use. Collembola communities in plantations were characterized by high abundance of species with sensory- and mobility-associated traits, suggesting that such traits help soildwelling arthropods in colonising plantations. Water content and $\mathrm{pH}$ were identified as environmental factors associated with species and trait composition of Collembola communities across land-use systems and layers. Further, Collembola communities likely were also structured by top-down control by micro- and macropredators (gamasid mites and spiders). However, environmental factors explained only up to $13.6 \%$ of Collembola community composition, suggesting that factors not included in this study may be more important. Overall, conversion of rainforest into plantation systems altered the composition of Collembola communities with potential consequences for decomposition processes and other ecosystem services they provide. To better understand the community assembly and functioning of tropical Collembola communities, studies on the ecology and genetic diversity of pantropical genera are needed.

\section{Acknowledgements}

This study was funded by the Deutsche Forschungsgemeinschaft (DFG, German Research Foundation) - project number 192626868 - SFB 990 in the framework of the collaborative German - Indonesian research project CRC990. We thank the State Ministry of Research and Technology of Indonesia (RISTEK), the Indonesian Institute of Sciences (LIPI), Ministry of Forestry (PHKA) and Restoration Ecosystem Indonesia Harapan for the permits. Sampling of animals used in this study was based on Collection permits No. S.07/KKH2/2013 and No. 2841/IPH.1/KS.02.04/X/2016 issued by the Indonesian Ministry of Forestry (PHKA). We thank Indonesian organizations and farmers for granting access to the sampling plots and use of their properties. We acknowledge support of the Open Access Publication Funds of the University of Göttingen. Special gratitude goes to Feng Zhang, Louis Deharveng and Anne Bedos for verification of Collembola morphospecies identification. We also thank Penelope Greenslade for sharing her unpublished drafts of the key on Indonesian Collembola. 
Chapter 3

\section{Appendix}

Appendix Table 3.1. Mean values of environmental factors in the litter and soil layer in the four land-use systems studied (rainforest, jungle rubber, rubber and oil palm plantations) in the years 2013 and 2016

\begin{tabular}{|c|c|c|c|c|c|c|c|c|c|c|c|c|c|}
\hline & Year & $\begin{array}{l}\text { Water } \\
\text { content } \\
(\%)\end{array}$ & $\mathrm{pH}$ & $\begin{array}{l}\text { C-to-N } \\
\text { ratio }\end{array}$ & $\begin{array}{l}\text { NLFA } \\
16: 1 \omega 5 \mathrm{c} \\
\text { (c nmol/g) }\end{array}$ & $\begin{array}{l}\text { PLFA } \\
18: 2 \omega 6,9 \\
(\%)\end{array}$ & $\begin{array}{l}\text { PLFA } \\
20: 5 \omega 3(\%)\end{array}$ & $\begin{array}{l}\mathrm{Gr}^{+} \\
\text {bacteria } \\
(\%)\end{array}$ & $\begin{array}{l}\mathrm{Gr}^{-} \\
\text {bacteria } \\
(\%)\end{array}$ & $\begin{array}{l}\text { Araneae } \\
\text { log10 } \\
\text { (ind/sample) }\end{array}$ & $\begin{array}{l}\text { Formicidae } \\
\text { log10 } \\
\text { (ind/sample) }\end{array}$ & $\begin{array}{l}\text { Mesostigmata } \\
\text { log10 } \\
\text { (ind/sample) }\end{array}$ & $\begin{array}{l}\text { Oribatida } \\
\text { log10 } \\
\text { (ind/sample) }\end{array}$ \\
\hline \multicolumn{14}{|l|}{ LItter layer } \\
\hline Rainforest & $2013(n=8)$ & 184.0 & 4.3 & 31.9 & 6.7 & 18.4 & 0.7 & 33.8 & 30.4 & 0.32 & 0.73 & 1.53 & 2.09 \\
\hline Rainforest & $2016(n=11)$ & 71.61 & 3.8 & 25.1 & 11.4 & 17.7 & 1.4 & 46.3 & 40.2 & 0.19 & 0.37 & 1.13 & 1.91 \\
\hline Jungle rubber & $2013(n=8)$ & 198.0 & 5.1 & 27.1 & 21.4 & 18.1 & 0.3 & 36.4 & 28.2 & 0.07 & 0.13 & 1.10 & 2.03 \\
\hline Oil palm & $2013(n=7)$ & 149.4 & 5.4 & 29.4 & 13.9 & 17.4 & 0.3 & 37.5 & 27.9 & 0.04 & 0.15 & 0.99 & 1.56 \\
\hline Oil palm & $2016(n=11)$ & 35.3 & 5.1 & 22.1 & 7.2 & 15.2 & 1.4 & 46.9 & 37.6 & 0 & 0 & 0.82 & 1.32 \\
\hline Rubber & $2013(n=7)$ & 116.1 & 5.5 & 29.5 & 15.1 & 20.7 & 0.7 & 39.9 & 24.8 & 0 & 0.51 & 1.07 & 1.72 \\
\hline Rubber & $2016(n=11)$ & 41.0 & 5.3 & 26.7 & 8.3 & 20.8 & 1.4 & 40.5 & 38.6 & 0.08 & 0.22 & 0.85 & 1.28 \\
\hline \multicolumn{14}{|l|}{ Soil layer } \\
\hline Rainforest & $2013(n=8)$ & 71.7 & 3.7 & 15.1 & 7.3 & 7.8 & 0.3 & 58.4 & 37.8 & 0.35 & 1.03 & 0.81 & 1.08 \\
\hline Rainforest & $2016(n=12)$ & 73.6 & 3.9 & 14.1 & 5.9 & 5.7 & 0.9 & 68.4 & 43.8 & 0.33 & 0.82 & 0.64 & 0.91 \\
\hline Jungle rubber & $2013(n=8)$ & 95.5 & 4.3 & 17.0 & 15.0 & 10.2 & 0.3 & 50.2 & 24.3 & 0.32 & 0.39 & 0.56 & 1.11 \\
\hline Oil palm & $2013(n=8)$ & 77.4 & 4.7 & 13.4 & 18.6 & 8.1 & 0.1 & 55.1 & 30.6 & 0.04 & 0.25 & 0.83 & 1.58 \\
\hline Oil palm & $2016(n=10)$ & 43.3 & 4.9 & 12. & 18.8 & 6.7 & 0.3 & 72.1 & 29.1 & 0.19 & 0.62 & 1.14 & 1.24 \\
\hline Rubber & $2013(n=8)$ & 59.0 & 4.3 & 11.9 & 13.9 & 8.8 & 0.1 & 45.5 & 21.7 & 0.13 & 0.59 & 0.88 & 1.38 \\
\hline Rubber & $2016(n=12)$ & 39.8 & 5.0 & 14.5 & 5.9 & 7.7 & 0.5 & 65.1 & 30.7 & 0.29 & 0.52 & 0.58 & 0.81 \\
\hline
\end{tabular}

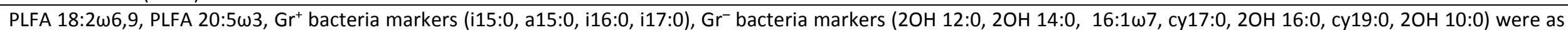

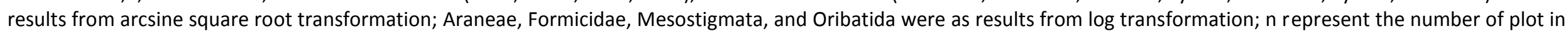
each year. 
Cluster Dendrogram

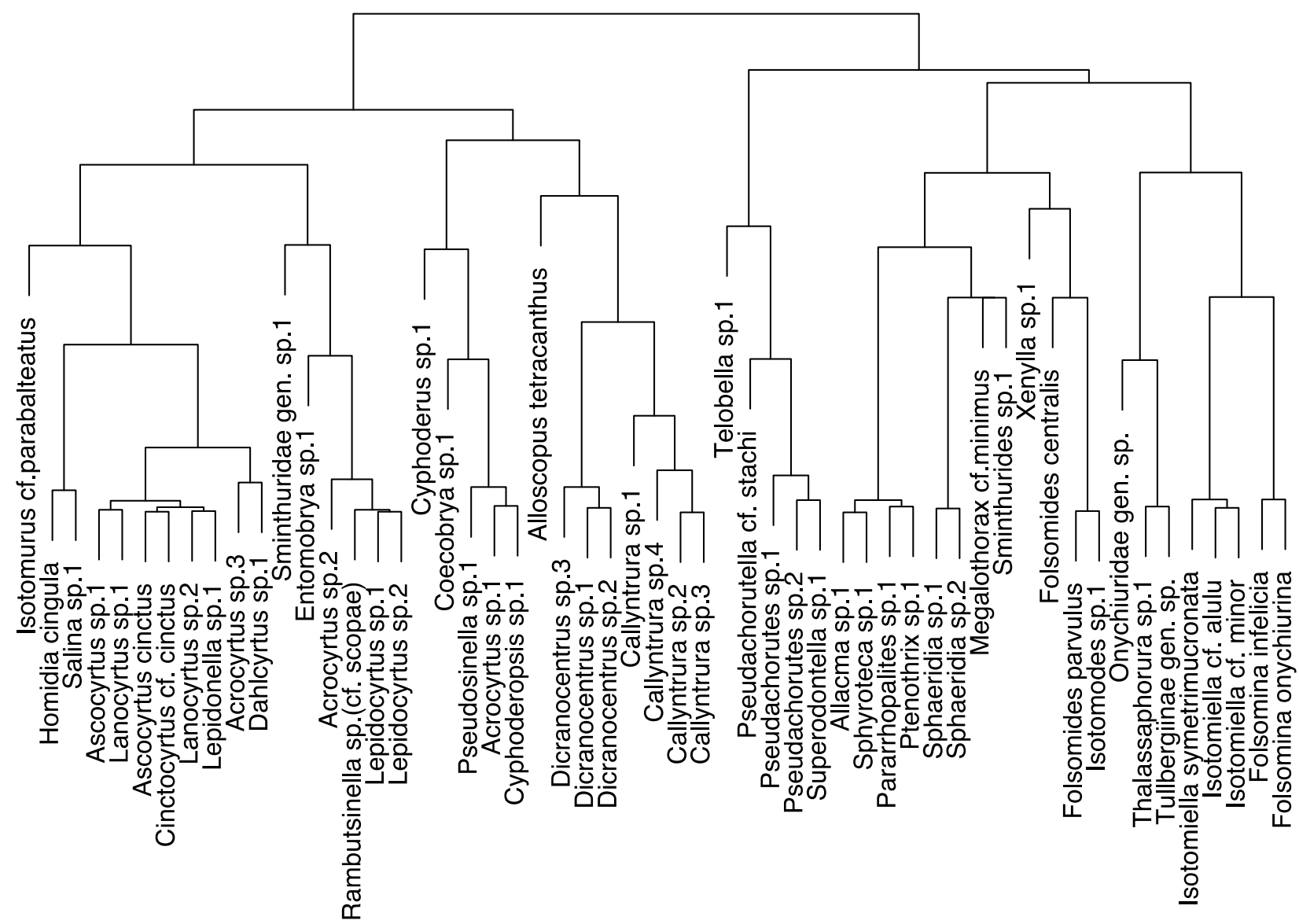

Appendix Figure 3.1. Hierarchical clustering of species based on their trait values. 
Appendix Table 3.2. Selected model and results for density based on region, system, layer, plot and year

\begin{tabular}{|c|c|c|c|c|c|c|c|}
\hline Df & $\mathrm{AIC}$ & $\mathrm{BIC}$ & $\operatorname{logLik}$ & deviance & Chisq & Chi Df & $\operatorname{Pr}(>$ Chisq $)$ \\
\hline 11 & 1360.1 & 1391.9 & -669.0 & 1338.1 & 2.0 & 0 & 0.0000 \\
\hline \multicolumn{8}{|c|}{ Results of the selected model for density per area } \\
\hline & & & Chisq & Df & \multicolumn{3}{|c|}{$\operatorname{Pr}(>$ Chisq $)$} \\
\hline Lay & & & 2.7 & 1 & \multicolumn{3}{|c|}{0.1025} \\
\hline Sys & $\mathrm{em}$ & & 6.7 & 3 & \multicolumn{3}{|c|}{0.0833} \\
\hline Lan & dscape & & 3.5 & 1 & \multicolumn{3}{|c|}{0.0627} \\
\hline Lay & er:System & & 11.8 & 3 & \multicolumn{3}{|c|}{0.0081} \\
\hline
\end{tabular}

\begin{tabular}{lllll}
\hline \multicolumn{6}{l}{ Results of Tukey } & test for density of system and layer & \\
\hline System & Layer & Mean & SD & Letter \\
\hline Jungle Rubber & Litter & 73.5 & 56.8 & $\mathrm{Ab}$ \\
Jungle Rubber & Soil & 32.6 & 22.0 & $\mathrm{Ab}$ \\
Oil Palm & Litter & 36.2 & 65.7 & $\mathrm{~B}$ \\
Oil Palm & Soil & 77.4 & 51.5 & $\mathrm{~A}$ \\
Rainforest & Litter & 71.0 & 61.2 & $\mathrm{ab}$ \\
Rainforest & Soil & 84.0 & 124.5 & $\mathrm{ab}$ \\
Rubber & Litter & 38.7 & 31.6 & $\mathrm{ab}$ \\
Rubber & Soil & 47.7 & 23.9 & $\mathrm{ab}$ \\
\hline
\end{tabular}

Appendix Table 3.3 Selected model and results for species richness based on landscape, system, layer, plot and year

Model: Variable $\sim 1+(\text { Layer }+ \text { System })^{\wedge} 2+$ Year $+(1 \mid$ YearPlot $)$

$\begin{array}{llllll}\text { Df } & \text { AIC } & \text { BIC } & \operatorname{logLik} & \text { Deviance Chisq } & \text { Chi } \\ \text { Df } & \operatorname{Pr}(>\text { Chisq })\end{array}$

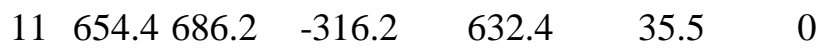
0.0000

Results of the selected model for species richness

\begin{tabular}{lccc}
\hline & Chisq & Df & $\operatorname{Pr}(>$ Chisq) \\
\hline Layer & 1.4 & 1 & 0.2297 \\
System & 7.5 & 3 & 0.0588 \\
Year & 48.7 & 1 & 0.0000 \\
Layer:System & 14.5 & 3 & 0.0023 \\
\hline
\end{tabular}

Results of Tukey test for species richness of system and layer

\begin{tabular}{lllll}
\hline System & Layer & Mean & SD & Letter \\
\hline Junggle Rubber & Litter & 6.75 & 2.71 & $\mathrm{ab}$ \\
Junggle Rubber & Soil & 6.00 & 3.38 & $\mathrm{ab}$ \\
Oil Palm & Litter & 3.68 & 2.76 & $\mathrm{~b}$ \\
Oil Palm & Soil & 6.68 & 3.57 & $\mathrm{a}$ \\
Rainforest & Litter & 6.95 & 5.14 & $\mathrm{a}$ \\
Rainforest & Soil & 5.35 & 3.09 & $\mathrm{ab}$ \\
Rubber & Litter & 4.30 & 2.71 & $\mathrm{ab}$ \\
Rubber & Soil & 5.10 & 2.22 & $\mathrm{ab}$ \\
\hline
\end{tabular}


Appendix Table 3.4. Selected model and results for functional diversity based on landscape, system, layer, plot and year

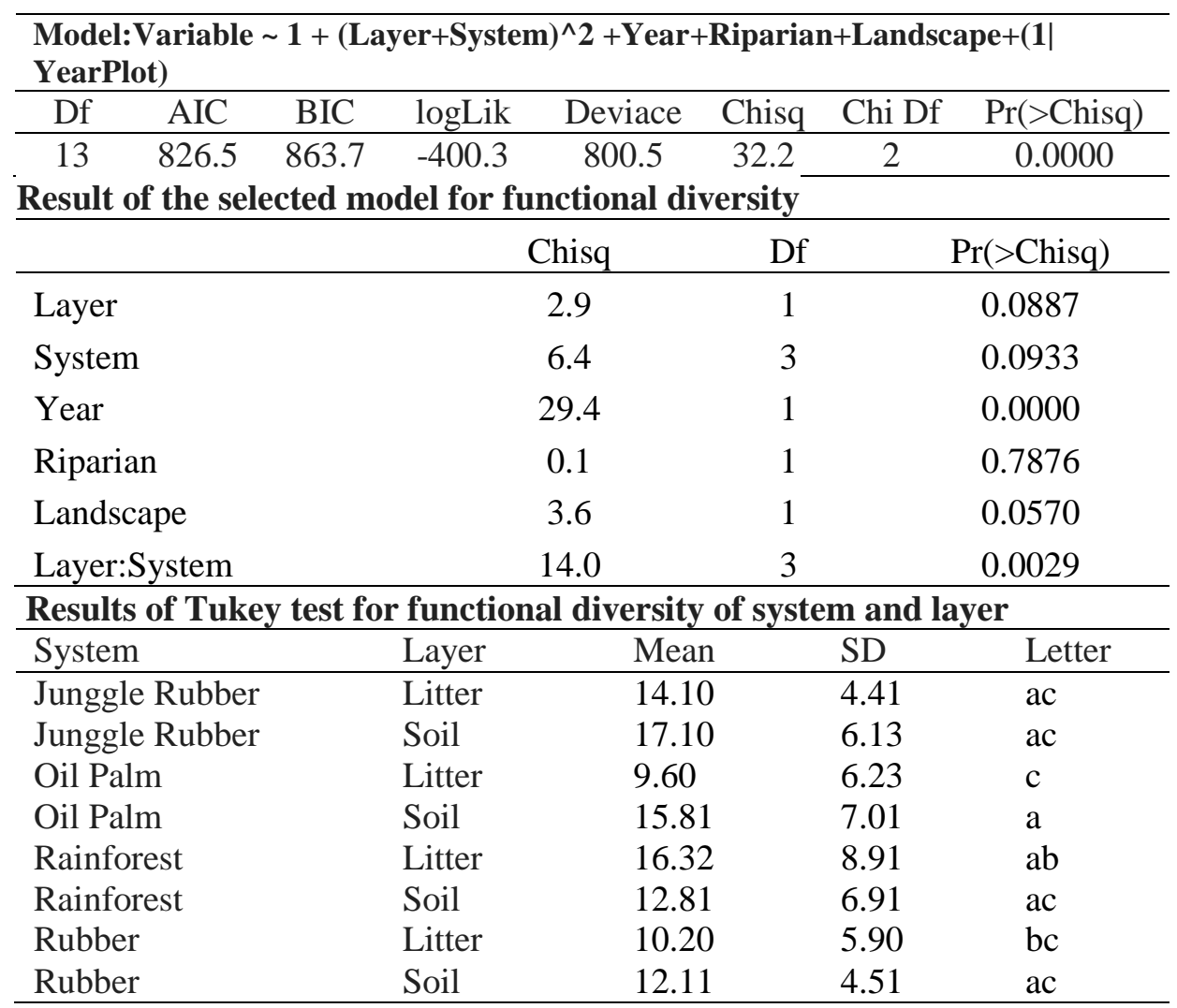

Appendix Table 3.5. Selected model and results for density per gram carbon based on region, system, layer, plot and year

\begin{tabular}{|c|c|c|c|c|c|c|}
\hline \multicolumn{7}{|c|}{$\begin{array}{l}\left.\text { Model :Variable } \sim 1+\text { Layer + System })^{\wedge} \text { 2+Riparian+(1|Year }\right)+(1 \mid \\
\text { Year/Plot)+(1|Landscape })\end{array}$} \\
\hline Df AIC & $\mathrm{BIC}$ & $\log \operatorname{Lik}$ & Deviance & Chisq & Chi Df & $\operatorname{Pr}(>$ Chisq $)$ \\
\hline $11 \quad 1275.7$ & 1307.5 & 626.8 & 1253.7 & 6.7 & 0 & 0.0000 \\
\hline \multicolumn{7}{|c|}{ Result of the selected model for density per gram of carbon } \\
\hline & & \multicolumn{2}{|r|}{ Chisq } & \multicolumn{2}{|r|}{ Df } & $\operatorname{Pr}(>$ Chisq) \\
\hline \multicolumn{2}{|l|}{ Layer } & \multicolumn{2}{|r|}{73.6} & \multicolumn{2}{|r|}{1} & 0.0000 \\
\hline \multicolumn{2}{|l|}{ System } & \multicolumn{2}{|r|}{4.5} & \multicolumn{2}{|r|}{3} & 0.2089 \\
\hline \multicolumn{2}{|l|}{ Year } & \multicolumn{2}{|r|}{7.5} & \multicolumn{2}{|r|}{1} & 0.0061 \\
\hline \multicolumn{2}{|l|}{ Layer:System } & \multicolumn{2}{|r|}{10.3} & \multicolumn{2}{|r|}{3} & 0.0162 \\
\hline
\end{tabular}

Results of Tukey test for density per gram carbon of system and layer

\begin{tabular}{llccc}
\hline \multicolumn{1}{c}{ System } & Layer & Mean & SD & Letter \\
\hline Junggle Rubber & Litter & 9.11 & 7.22 & $\mathrm{a}$ \\
Junggle Rubber & Soil & 0.57 & 0.32 & $\mathrm{~d}$ \\
Oil Palm & Litter & 5.75 & 5.94 & $\mathrm{a}$ \\
Oil Palm & Soil & 1.70 & 1.13 & $\mathrm{bc}$ \\
Rainforest & Litter & 4.22 & 3.47 & $\mathrm{ab}$ \\
Rainforest & Soil & 1.42 & 1.99 & $\mathrm{~cd}$ \\
Rubber & Litter & 4.19 & 3.60 & $\mathrm{ab}$ \\
Rubber & Soil & 1.09 & 0.57 & $\mathrm{~cd}$ \\
\hline
\end{tabular}


Appendix Table 3.6. Selected model and results for functional dispersion based on region, system, layer, plot and year

\begin{tabular}{|c|c|c|c|c|c|c|c|}
\hline \multicolumn{8}{|c|}{ Model: Variable $\sim 1+(\text { Layer+System })^{\wedge} 2+$ Year+(1| YearPlot $)$} \\
\hline Df & AIC & BIC & logLik & Deviace & Chisq & Chi Df & $\operatorname{Pr}(>$ Chisq $)$ \\
\hline 11 & -341.6 & -310.2 & 181.8 & -363.6 & 3.80 & 2 & 0.00 \\
\hline \multicolumn{8}{|c|}{ Results of the selected model for functional dispersion } \\
\hline & & & & Chisq & & & $\operatorname{Pr}(>$ Chisq $)$ \\
\hline \multicolumn{3}{|c|}{ Layer } & & 0.1 & & & 0.8981 \\
\hline \multicolumn{3}{|c|}{ System } & & 1.1 & & & 0.7601 \\
\hline \multicolumn{3}{|c|}{ Year } & & 3.8 & & & 0.0510 \\
\hline \multicolumn{3}{|c|}{ Layer:System } & & 12.6 & & 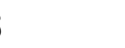 & 0.0053 \\
\hline
\end{tabular}


Chapter 3

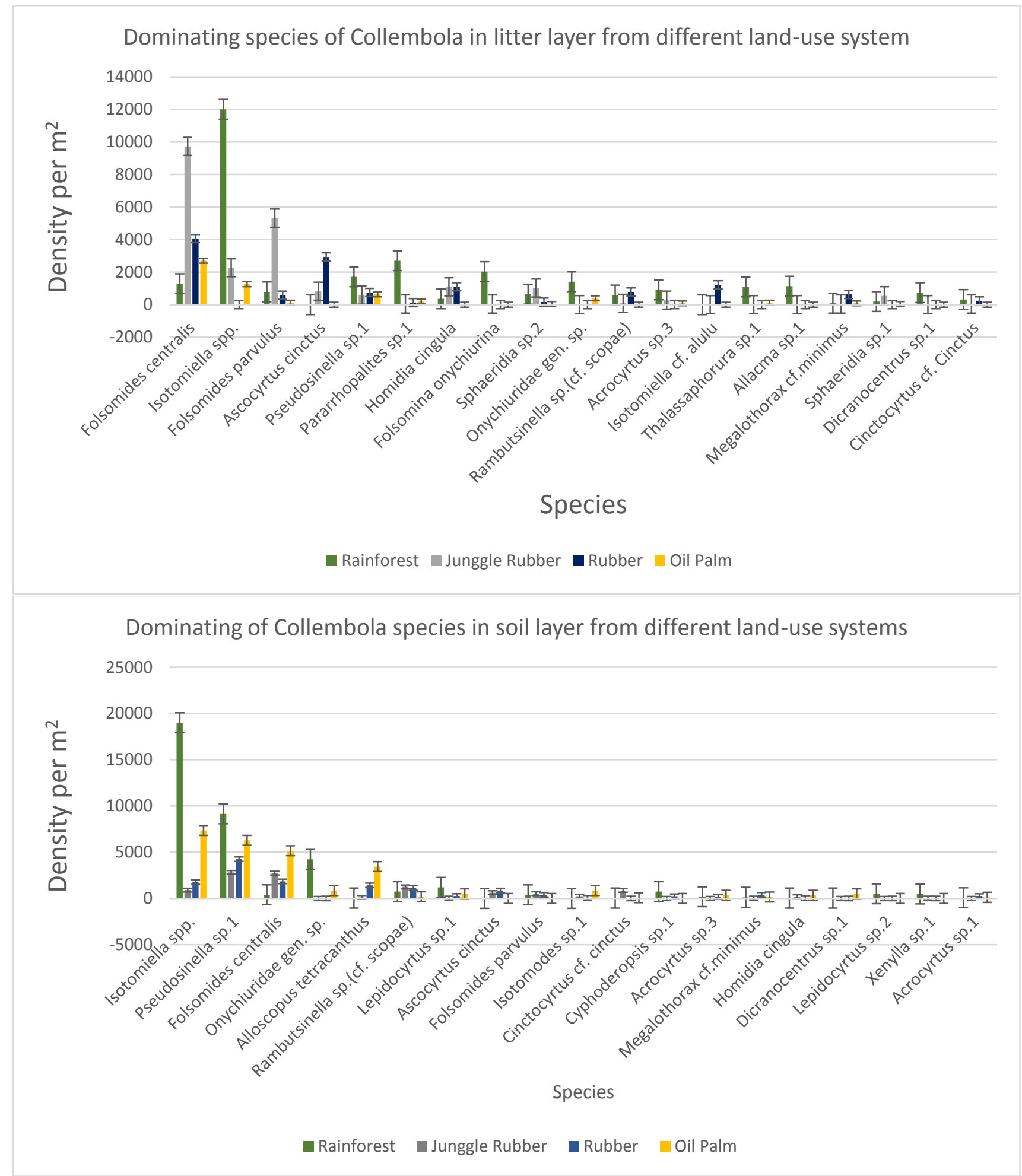

Appendix Figure 3.2. Dominating species of Collembola in litter and soil layer from different landuse systems. 
Land use $\bullet$ Jungle rubber $\bullet$ Oil palm $\bullet$ Rainforest $\bullet$ Rubber

a. Species litter

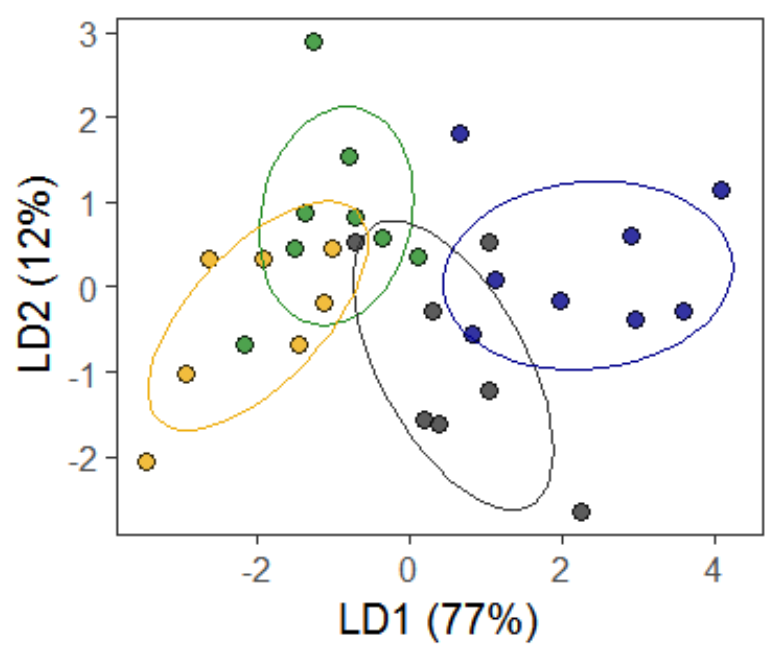

c. Species soil

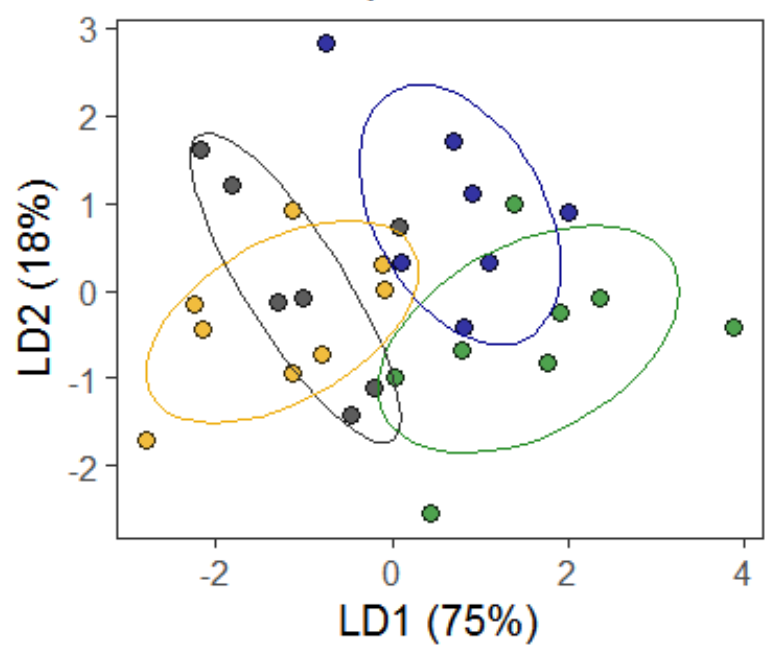

b. Trait litter

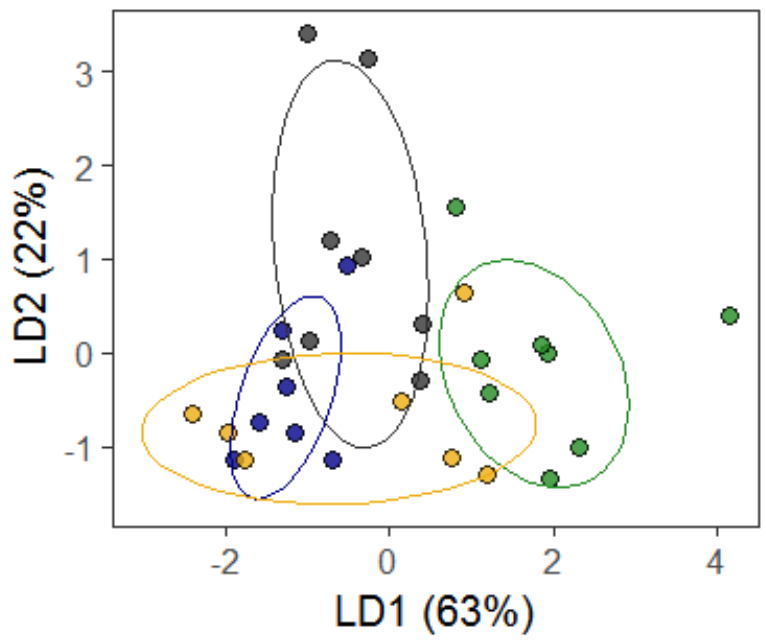

d. Trait soil

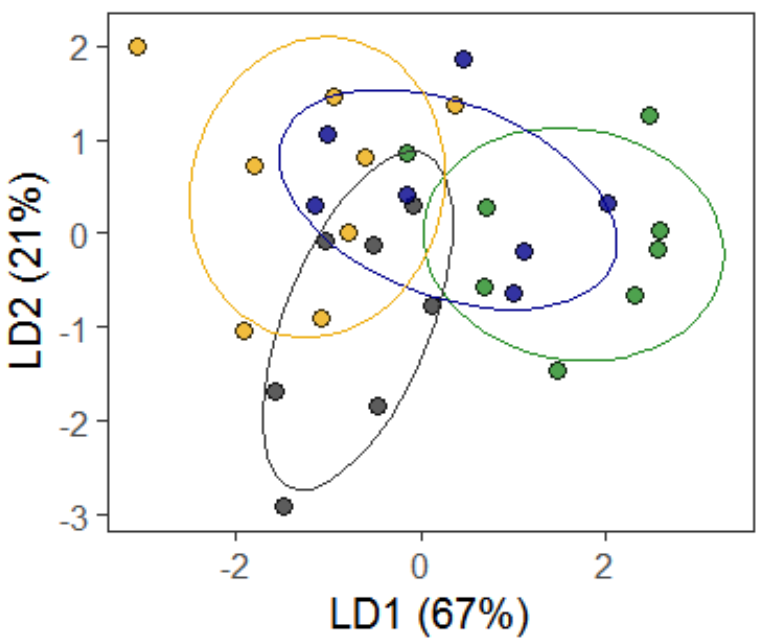

Appendix Figure 3.3. Linear discriminant analysis of species (a, c) and trait composition (b, d) of Collembola communities in the litter (a, b) and soil layer (c, d) in 2013. Land-use system was used as grouping variable. Each point represents a sample. 
a. Species litter

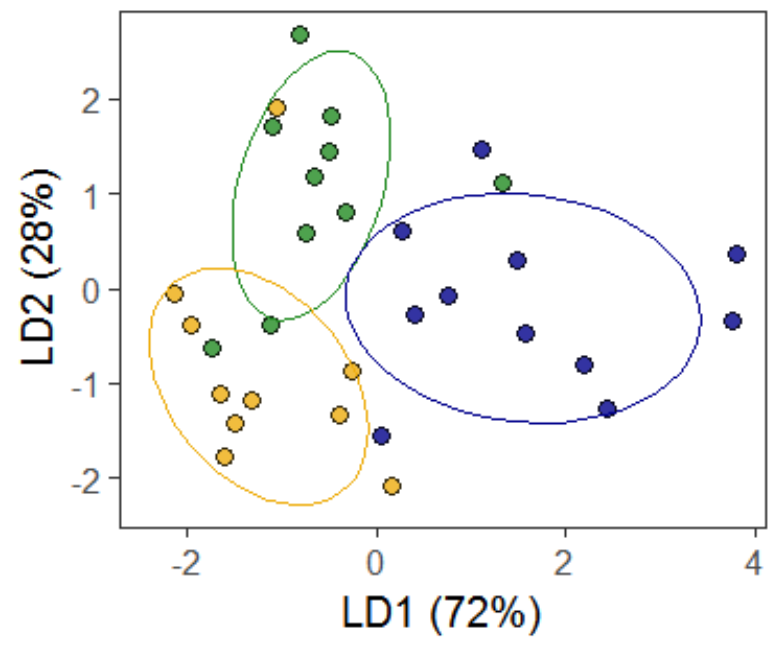

C. Species soil

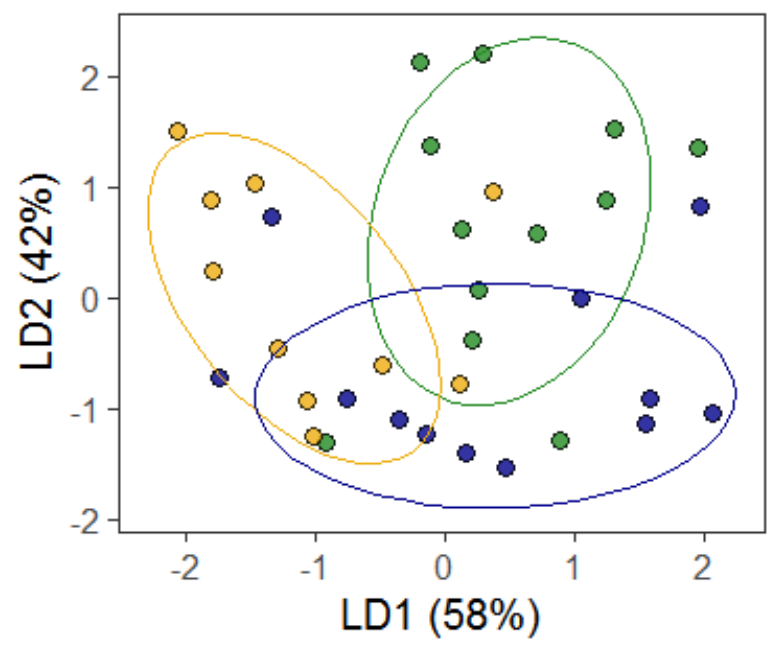

b. Trait litter

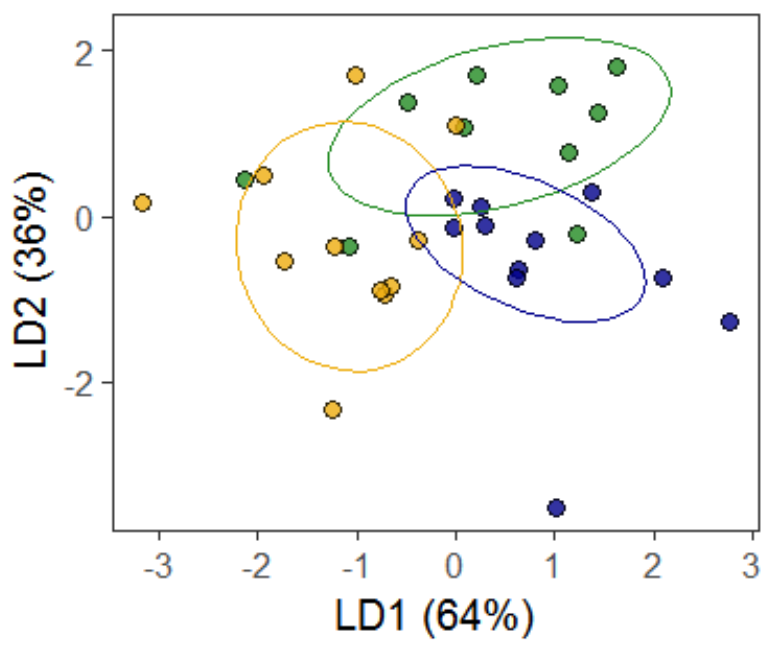

d. Trait soil

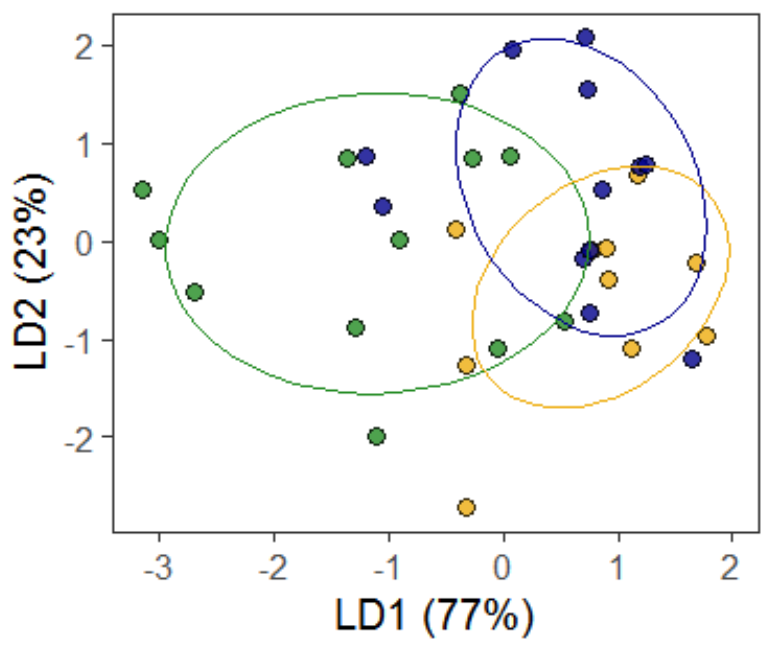

Appendix Figure 3.4. Linear discriminant analysis of species $(a, c)$ and trait composition $(b, d)$ of Collembola communities in the litter (a, b) and soil layer (c, d) in 2016. Land-use system was used as grouping variable. Each point represents a sample. 
Chapter 3

Appendix Table 3.7. Mahalanobis distances from LDA of species composition in litter and soil layer

\begin{tabular}{lccccc}
\hline Litter layer & F-value & df1 & df2 & p-alue & MD $^{2}$ \\
\hline Rainforest - Jungle rubber & 6.57 & 3 & 22 & 0.0024 & 3.2315 \\
Rainforest - Rubber & 14.87 & 3 & 32 & 0.0001 & 1.4898 \\
Rainforest - Oil palm & 4.73 & 3 & 31 & 0.0078 & 3.1200 \\
Jungle rubber - Rubber & 4.50 & 3 & 22 & 0.0130 & 1.6731 \\
Jungle rubber - Oil palm & 5.35 & 3 & 21 & 0.0067 & 1.9256 \\
Rubber - Oil palm & NaN & 3 & 14 & NaN & 2.5382 \\
\hline Soil layer & & & & & \\
\hline Rainforest - Jungle rubber & 5.18 & 3 & 23 & 0.0069 & 2.2691 \\
Rainforest - Rubber & 8.00 & 3 & 36 & 0.0003 & 1.7148 \\
Rainforest - Oil palm & 9.30 & 3 & 33 & 0.0001 & 1.8029 \\
Jungle rubber - Rubber & 3.08 & 3 & 23 & 0.0471 & 1.4614 \\
Jungle rubber - Oil palm & 3.00 & 3 & 20 & 0.0544 & 1.7851 \\
Rubber - Oil palm & NA & 3 & 16 & NA & 1.6884 \\
\hline
\end{tabular}

Appendix Table 3.8. Mahalanobis distances from LDA of trait composition in litter and soil layer

\begin{tabular}{lccccc}
\hline Litter layer & F-value & df1 & df2 & p-value & MD $^{2}$ \\
\hline Rainforest - Jungle rubber & 4.74 & 3 & 22 & 0.0106 & 2.2898 \\
Rainforest - Rubber & 5.73 & 3 & 32 & 0.0029 & 2.5403 \\
Rainforest - Oil palm & 9.99 & 3 & 32 & 0.0001 & 1.7000 \\
Jungle rubber - Rubber & 4.62 & 3 & 22 & 0.0117 & 2.4590 \\
Jungle rubber - Oil palm & 4.53 & 3 & 22 & 0.0127 & 2.5188 \\
Rubber - Oil palm & NA & 3 & 14 & NA & 1.8473 \\
\hline Soil layer & & & & & \\
\hline Rainforest - Jungle rubber & 6.00 & 3 & 23 & 0.0035 & 3.2876 \\
Rainforest - Rubber & 9.35 & 3 & 36 & 0.0001 & 1.8002 \\
Rainforest - Oil palm & 7.90 & 3 & 33 & 0.0004 & 1.9245 \\
Jungle rubber - Rubber & 4.13 & 3 & 23 & 0.0175 & 2.2041 \\
Jungle rubber - Oil palm & 7.63 & 3 & 20 & 0.0013 & 2.3269 \\
Rubber - Oil palm & NA & 3 & 16 & NA & 1.0510 \\
\hline
\end{tabular}


Chapter 3

\section{Supplementary Material}

Supplementary Table S3.1. Full list of species and traits

\begin{tabular}{|c|c|c|c|c|c|c|c|c|c|c|c|}
\hline No. & Species & Pigmentation & Ocelli & Antennae & Furca & Abdomen & Scales & PAO & Mouthparts & $\begin{array}{l}\text { Empodial } \\
\text { appendage }\end{array}$ & Size Class \\
\hline 1 & Acrocyrtus sp. 1 & Absent & $>=6+6$ & Normal, 4 segments & Whip-shaped & $\begin{array}{l}\text { Abdomen IV } \\
\text { elongated }\end{array}$ & $\begin{array}{l}\text { Scales } \\
\text { smooth }\end{array}$ & $\begin{array}{l}\text { PAO } \\
\text { absent }\end{array}$ & Chewing & Present & Medium size \\
\hline 2 & Acrocyrtus sp. 2 & Diffuse & $>=6+6$ & Normal, 4 segments & Whip-shaped & $\begin{array}{l}\text { Abdomen IV } \\
\text { elongated }\end{array}$ & $\begin{array}{l}\text { Scales } \\
\text { smooth }\end{array}$ & $\begin{array}{l}\text { PAO } \\
\text { absent }\end{array}$ & Chewing & Present & Large size \\
\hline 3 & Acrocyrtus sp. 3 & Pattern & $>=6+6$ & Normal, 4 segments & Whip-shaped & $\begin{array}{l}\text { Abdomen IV } \\
\text { elongated }\end{array}$ & $\begin{array}{l}\text { Scales } \\
\text { smooth }\end{array}$ & $\begin{array}{l}\text { PAO } \\
\text { absent }\end{array}$ & Chewing & Present & Large size \\
\hline 4 & Allacma sp.1 & Pattern & $>=6+6$ & $\begin{array}{l}\text { Antennae III and/or IV } \\
\text { subdivided }\end{array}$ & Straight & Spherical body & Absent & $\begin{array}{l}\text { PAO } \\
\text { absent }\end{array}$ & Chewing & Present & Small size \\
\hline 5 & Dahlcyrtus sp. 1 & Pattern & $>=6+6$ & Normal, 4 segments & Whip-shaped & $\begin{array}{l}\text { Abdomen IV } \\
\text { elongated }\end{array}$ & $\begin{array}{l}\text { Scales } \\
\text { smooth }\end{array}$ & $\begin{array}{l}\text { PAO } \\
\text { absent }\end{array}$ & Chewing & Present & Large size \\
\hline 6 & Ascocyrtus cinctus & Pattern & $>=6+6$ & Normal, 4 segments & Whip-shaped & $\begin{array}{l}\text { Abdomen IV } \\
\text { elongated }\end{array}$ & $\begin{array}{l}\text { Scales } \\
\text { smooth }\end{array}$ & $\begin{array}{l}\text { PAO } \\
\text { absent }\end{array}$ & Chewing & Present & Medium size \\
\hline 7 & Ascocyrtus sp. 1 & Pattern & $>=6+6$ & Normal, 4 segments & Whip-shaped & $\begin{array}{l}\text { Abdomen IV } \\
\text { elongated }\end{array}$ & $\begin{array}{l}\text { Scales } \\
\text { smooth }\end{array}$ & $\begin{array}{l}\text { PAO } \\
\text { absent }\end{array}$ & Chewing & Present & Medium size \\
\hline 8 & Callyntrura sp. 1 & Pattern & $>=6+6$ & Antennae I very long & Long cylindric & $\begin{array}{l}\text { Abdomen IV } \\
\text { elongated }\end{array}$ & $\begin{array}{l}\text { Scales } \\
\text { smooth }\end{array}$ & $\begin{array}{l}\text { PAO } \\
\text { absent }\end{array}$ & Chewing & Present & Large size \\
\hline 9 & Callyntrura sp. 2 & Pattern & $>=6+6$ & Antennae I very long & Long cylindric & $\begin{array}{l}\text { Abdomen IV } \\
\text { elongated }\end{array}$ & $\begin{array}{l}\text { Scales } \\
\text { smooth }\end{array}$ & $\begin{array}{l}\text { PAO } \\
\text { absent }\end{array}$ & Chewing & Present & Medium size \\
\hline 10 & Callyntrura sp.3 & Pattern & $>=6+6$ & Antennae I very long & Long cylindric & $\begin{array}{l}\text { Abdomen IV } \\
\text { elongated }\end{array}$ & $\begin{array}{l}\text { Scales } \\
\text { smooth }\end{array}$ & $\begin{array}{l}\text { PAO } \\
\text { absent }\end{array}$ & Chewing & Present & Medium size \\
\hline 11 & Callyntrura sp.4 & Pattern & $>=6+6$ & Antennae I very long & Long cylindric & $\begin{array}{l}\text { Abdomen IV } \\
\text { elongated }\end{array}$ & $\begin{array}{l}\text { Scales } \\
\text { smooth }\end{array}$ & $\begin{array}{l}\text { PAO } \\
\text { absent }\end{array}$ & Chewing & Present & Large size \\
\hline 12 & Coecobrya sp.1 & Absent & Absent & Normal, 4 segments & Whip-shaped & $\begin{array}{l}\text { Abdomen IV } \\
\text { elongated }\end{array}$ & Absent & $\begin{array}{l}\text { PAO } \\
\text { absent }\end{array}$ & Chewing & Present & Medium size \\
\hline 13 & Cyphoderopsis sp.1 & Absent & Absent & Normal, 4 segments & Long cylindric & $\begin{array}{l}\text { Abdomen IV } \\
\text { elongated }\end{array}$ & $\begin{array}{l}\text { Scales } \\
\text { smooth }\end{array}$ & $\begin{array}{l}\text { PAO } \\
\text { absent }\end{array}$ & Chewing & Present & Medium size \\
\hline 14 & Cyphoderus sp.1 & Absent & Absent & Normal, 4 segments & Straight & $\begin{array}{l}\text { Abdomen IV } \\
\text { elongated }\end{array}$ & $\begin{array}{l}\text { Scales } \\
\text { smooth }\end{array}$ & $\begin{array}{l}\text { PAO } \\
\text { absent }\end{array}$ & Chewing & Present & Medium size \\
\hline 15 & Dicranocentrus sp.1 & Pattern & $>=6+6$ & $\begin{array}{l}\text { Antennae I and/or II } \\
\text { subdivided }\end{array}$ & Whip-shaped & Not modified & $\begin{array}{l}\text { Scales } \\
\text { smooth }\end{array}$ & $\begin{array}{l}\text { PAO } \\
\text { absent }\end{array}$ & Chewing & Present & Large size \\
\hline 16 & Dicranocentrus sp.2 & Pattern & $>=6+6$ & $\begin{array}{l}\text { Antennae I and/or II } \\
\text { subdivided }\end{array}$ & Whip-shaped & Not modified & $\begin{array}{l}\text { Scales } \\
\text { smooth }\end{array}$ & $\begin{array}{l}\text { PAO } \\
\text { absent }\end{array}$ & Chewing & Present & Medium size \\
\hline 17 & Dicranocentrus sp.3 & Pattern & $>=6+6$ & $\begin{array}{l}\text { Antennae I and/or II } \\
\text { subdivided }\end{array}$ & Whip-shaped & Not modified & $\begin{array}{l}\text { Scales } \\
\text { smooth }\end{array}$ & $\begin{array}{l}\text { PAO } \\
\text { absent }\end{array}$ & Chewing & Present & Medium size \\
\hline 18 & Entomobrya $\mathrm{sp} .1$ & Diffuse & $>=6+6$ & Normal, 4 segments & Whip-shaped & $\begin{array}{l}\text { Abdomen IV } \\
\text { elongated }\end{array}$ & Absent & $\begin{array}{l}\text { PAO } \\
\text { absent }\end{array}$ & Chewing & Present & Small size \\
\hline
\end{tabular}


Chapter 3

\begin{tabular}{|c|c|c|c|c|c|c|c|c|c|c|c|}
\hline 19 & Folsomides centralis & Diffuse & $>=6+6$ & Normal, 4 segments & Straight & Not modified & Absent & $\begin{array}{l}\text { PAO } \\
\text { simple }\end{array}$ & Chewing & Present & Small size \\
\hline 20 & Folsomides parvulus & Absent & $2+2$ & Normal, 4 segments & Straight & Not modified & Absent & $\begin{array}{l}\text { PAO } \\
\text { simple }\end{array}$ & Chewing & Present & Small size \\
\hline 21 & Folsomina infelicia & Absent & Absent & Normal, 4 segments & Straight & $\begin{array}{l}\text { Abdomen IV to } \\
\text { VI or V to VI } \\
\text { fused }\end{array}$ & Absent & $\begin{array}{l}\text { PAO } \\
\text { absent }\end{array}$ & Chewing & Present & Small size \\
\hline 22 & Folsomina onychiurina & Absent & Absent & Normal, 4 segments & Straight & $\begin{array}{l}\text { Abdomen IV to } \\
\text { VI or V to VI } \\
\text { fused }\end{array}$ & Absent & $\begin{array}{l}\text { PAO } \\
\text { absent }\end{array}$ & Chewing & Present & Medium size \\
\hline 23 & Alloscopus tetracanthus & Absent & Absent & $\begin{array}{l}\text { Antennae I and/or II } \\
\text { subdivided }\end{array}$ & Whip-shaped & Not modified & $\begin{array}{l}\text { Scales } \\
\text { smooth }\end{array}$ & $\begin{array}{l}\text { PAO } \\
\text { simple }\end{array}$ & Chewing & Present & Large size \\
\hline 24 & Homidia cingula & Pattern & $>=6+6$ & Normal, 4 segments & Whip-shaped & $\begin{array}{l}\text { Abdomen IV } \\
\text { elongated }\end{array}$ & Absent & $\begin{array}{l}\text { PAO } \\
\text { absent }\end{array}$ & Chewing & Present & Large size \\
\hline 25 & Isotomiella cf. Alulu & Absent & Absent & Normal, 4 segments & Whip-shaped & $\begin{array}{l}\text { Abdomen IV to } \\
\text { VI or V to VI } \\
\text { fused }\end{array}$ & Absent & $\begin{array}{l}\text { PAO } \\
\text { absent }\end{array}$ & Chewing & Present & Small size \\
\hline 26 & Isotomiella cf. Minor & Absent & Absent & Normal, 4 segments & Whip-shaped & $\begin{array}{l}\text { Abdomen IV to } \\
\text { VI or V to VI } \\
\text { fused }\end{array}$ & Absent & $\begin{array}{l}\text { PAO } \\
\text { absent }\end{array}$ & Chewing & Present & Small size \\
\hline 27 & $\begin{array}{l}\text { Isotomiella } \\
\text { symetrimucronata }\end{array}$ & Absent & Absent & Normal, 4 segments & Whip-shaped & $\begin{array}{l}\text { Abdomen IV to } \\
\text { VI or V to VI } \\
\text { fused }\end{array}$ & Absent & $\begin{array}{l}\text { PAO } \\
\text { absent }\end{array}$ & Chewing & Present & Small size \\
\hline 28 & Isotomodes sp.1 & Absent & Absent & Normal, 4 segments & Straight & Not modified & Absent & $\begin{array}{l}\text { PAO } \\
\text { simple }\end{array}$ & Chewing & Present & Small size \\
\hline 29 & $\begin{array}{l}\text { Isotomurus cf. } \\
\text { Parabalteatus }\end{array}$ & Pattern & $>=6+6$ & Normal, 4 segments & Whip-shaped & Not modified & Absent & $\begin{array}{l}\text { PAO } \\
\text { simple }\end{array}$ & Chewing & Present & Medium size \\
\hline 30 & Lanocyrtus sp.1 & Pattern & $>=6+6$ & Normal, 4 segments & Whip-shaped & $\begin{array}{l}\text { Abdomen IV } \\
\text { elongated }\end{array}$ & $\begin{array}{l}\begin{array}{l}\text { Scales } \\
\text { smooth }\end{array} \\
\end{array}$ & $\begin{array}{l}\text { PAO } \\
\text { absent }\end{array}$ & Chewing & Present & Small size \\
\hline 31 & Lanocyrtus sp. 2 & Pattern & $>=6+6$ & Normal, 4 segments & Whip-shaped & $\begin{array}{l}\text { Abdomen IV } \\
\text { elongated }\end{array}$ & $\begin{array}{l}\text { Scales } \\
\text { smooth }\end{array}$ & $\begin{array}{l}\text { PAO } \\
\text { absent }\end{array}$ & Chewing & Present & Medium size \\
\hline 32 & Lepidocyrtus sp.1 & Diffuse & $>=6+6$ & Normal, 4 segments & Whip-shaped & $\begin{array}{l}\text { Abdomen IV } \\
\text { elongated }\end{array}$ & $\begin{array}{l}\begin{array}{l}\text { Scales } \\
\text { smooth }\end{array} \\
\end{array}$ & $\begin{array}{l}\text { PAO } \\
\text { absent }\end{array}$ & Chewing & Present & Medium size \\
\hline 33 & Lepidocyrtus sp. 2 & Diffuse & $>=6+6$ & Normal, 4 segments & Whip-shaped & $\begin{array}{l}\text { Abdomen IV } \\
\text { elongated }\end{array}$ & $\begin{array}{l}\begin{array}{l}\text { Scales } \\
\text { smooth }\end{array} \\
\end{array}$ & $\begin{array}{l}\text { PAO } \\
\text { absent }\end{array}$ & Chewing & Present & Medium size \\
\hline 34 & Lepidonella sp.1 & Pattern & $>=6+6$ & Normal, 4 segments & Long cylindric & $\begin{array}{l}\text { Abdomen IV } \\
\text { elongated }\end{array}$ & $\begin{array}{l}\begin{array}{l}\text { Scales } \\
\text { smooth }\end{array} \\
\end{array}$ & $\begin{array}{l}\text { PAO } \\
\text { absent }\end{array}$ & Chewing & Present & Medium size \\
\hline 35 & $\begin{array}{l}\text { Megalothorax cf. } \\
\text { Minimus }\end{array}$ & Absent & Absent & Normal, 4 segments & Straight & Spherical body & Absent & $\begin{array}{l}\text { PAO } \\
\text { absent }\end{array}$ & Chewing & Present & Small size \\
\hline 36 & Onychiuridae gen. sp. & Absent & Absent & Normal, 4 segments & Absent & Not modified & Absent & $\begin{array}{l}\mathrm{PAO} \\
\text { complex }\end{array}$ & Chewing & Present & Small size \\
\hline
\end{tabular}


Chapter 3

\begin{tabular}{|c|c|c|c|c|c|c|c|c|c|c|c|}
\hline 37 & Pararrhopalites sp. 1 & Pattern & $>=6+6$ & $\begin{array}{l}\text { Antennae III and/or IV } \\
\text { subdivided }\end{array}$ & Straight & Spherical body & Absent & $\begin{array}{l}\text { PAO } \\
\text { absent }\end{array}$ & Chewing & Present & Small size \\
\hline 38 & $\begin{array}{l}\text { Pseudachorutella cf. } \\
\text { Stachi }\end{array}$ & Intensive & $>=6+6$ & Normal, 4 segments & Straight & Not modified & Absent & $\begin{array}{l}\text { PAO } \\
\text { absent }\end{array}$ & piercing/sucking & Absent & Medium size \\
\hline 39 & Pseudachorutes sp.1 & Intensive & $>=6+6$ & Normal, 4 segments & Straight & Not modified & Absent & $\begin{array}{l}\text { PAO } \\
\text { complex }\end{array}$ & piercing/sucking & Absent & Medium size \\
\hline 40 & Pseudachorutes sp.2 & Intensive & $>=6+6$ & Normal, 4 segments & Straight & Not modified & Absent & $\begin{array}{l}\text { PAO } \\
\text { complex }\end{array}$ & piercing/sucking & Absent & Small size \\
\hline 41 & Pseudosinella sp.1 & Absent & Absent & Normal, 4 segments & Whip-shaped & $\begin{array}{l}\text { Abdomen IV } \\
\text { elongated }\end{array}$ & $\begin{array}{l}\text { Scales } \\
\text { smooth }\end{array}$ & $\begin{array}{l}\text { PAO } \\
\text { absent }\end{array}$ & Chewing & Present & Medium size \\
\hline 42 & Ptenothrix sp.1 & Pattern & $>=6+6$ & $\begin{array}{l}\text { Antennae IV shorter than } \\
\text { III }\end{array}$ & Straight & Spherical body & Absent & $\begin{array}{l}\text { PAO } \\
\text { absent }\end{array}$ & Chewing & Present & Medium size \\
\hline 43 & $\begin{array}{l}\text { Rambutsinella sp. (cf. } \\
\text { scopae) }\end{array}$ & Diffuse & $2+2$ & Normal, 4 segments & Whip-shaped & $\begin{array}{l}\text { Abdomen IV } \\
\text { elongated }\end{array}$ & $\begin{array}{l}\text { Scales } \\
\text { smooth }\end{array}$ & $\begin{array}{l}\text { PAO } \\
\text { absent }\end{array}$ & Chewing & Present & Medium size \\
\hline 44 & Salina sp.1 & Pattern & $>=6+6$ & Normal, 4 segments & Long cylindric & $\begin{array}{l}\text { Abdomen IV } \\
\text { elongated }\end{array}$ & Absent & $\begin{array}{l}\text { PAO } \\
\text { absent }\end{array}$ & Chewing & Present & Large size \\
\hline 45 & Sminthuridae gen. sp.1 & Diffuse & $>=6+6$ & Normal, 4 segments & Long cylindric & Spherical body & Absent & $\begin{array}{l}\text { PAO } \\
\text { absent }\end{array}$ & Chewing & Present & Small size \\
\hline 46 & Sminthurides sp.1 & Diffuse & $>=6+6$ & Normal, 4 segments & Straight & Spherical body & Absent & $\begin{array}{l}\text { PAO } \\
\text { absent }\end{array}$ & Chewing & Present & Small size \\
\hline 47 & Sphaeridia sp.1 & Intensive & $>=6+6$ & Normal, 4 segments & Straight & Spherical body & Absent & $\begin{array}{l}\text { PAO } \\
\text { absent }\end{array}$ & Chewing & Present & Small size \\
\hline 48 & Sphaeridia sp.2 & Intensive & $>=6+6$ & Normal, 4 segments & Straight & Spherical body & Absent & $\begin{array}{l}\text { PAO } \\
\text { absent }\end{array}$ & Chewing & Present & Small size \\
\hline 49 & Sphyroteca sp.1 & Pattern & $>=6+6$ & $\begin{array}{l}\text { Antennae III and/or IV } \\
\text { subdivided }\end{array}$ & Straight & Spherical body & Absent & $\begin{array}{l}\text { PAO } \\
\text { absent }\end{array}$ & Chewing & Present & Small size \\
\hline 50 & Superodontella sp.1 & Intensive & $5+5$ & Normal, 4 segments & Straight & Not modified & Absent & $\begin{array}{l}\text { PAO } \\
\text { complex }\end{array}$ & Not Chewing & Absent & Small size \\
\hline 51 & Telobella $\mathrm{sp} .1$ & Intensive & $2+2$ & Normal, 4 segments & Absent & Not modified & Absent & $\begin{array}{l}\text { PAO } \\
\text { absent }\end{array}$ & piercing/sucking & Absent & Large size \\
\hline 52 & Thalassaphorura sp.1 & Absent & Absent & Normal, 4 segments & Absent & Not modified & Absent & $\begin{array}{l}\text { PAO } \\
\text { complex }\end{array}$ & Chewing & Absent & Small size \\
\hline 53 & Tullbergiinae gen. sp. & Absent & Absent & Normal, 4 segments & Absent & Not modified & Absent & $\begin{array}{l}\text { PAO } \\
\text { complex }\end{array}$ & Chewing & Absent & Medium size \\
\hline 54 & Xenylla sp.1 & Diffuse & $4+4$ & Normal, 4 segments & Straight & Not modified & Absent & $\begin{array}{l}\text { PAO } \\
\text { absent }\end{array}$ & Chewing & Absent & Small size \\
\hline
\end{tabular}


Chapter 3

Supplementary Table S3.2. Species matrix of Collembola (individuals per plot) in litter layer

\begin{tabular}{|c|c|c|c|c|c|c|c|c|c|c|c|c|c|c|c|c|c|c|c|c|c|c|c|}
\hline Systems & Plot & Year & Acrl & Acr 2 & Allo & Call1 & Asco & Dicrl & Folce & Folspa & Homci & Isomi & Lep1 & Mega & Ony & Parl & Pseul & Ram & Sphal & Spha2 & Sphyl & Sup1 & Xen1 \\
\hline Rainforest & $\mathrm{BF} 1 \mathrm{~b}$ & 2013 & 1 & 12 & 0 & 2 & 3 & 1 & 0 & 19 & 0 & 21 & 0 & 0 & 2 & 4 & 1 & 4 & 0 & 0 & 2 & 0 & 0 \\
\hline Rainforest & $\mathrm{BF} 2 \mathrm{~b}$ & 2013 & 0 & 0 & 0 & 0 & 0 & 0 & 21 & 0 & 0 & 2 & 0 & 0 & 30 & 0 & 0 & 0 & 1 & 0 & 0 & 0 & 0 \\
\hline Rainforest & BF3b & 2013 & 0 & 10 & 0 & 1 & 0 & 4 & 3 & 0 & 9 & 0 & 0 & 1 & 13 & 4 & 10 & 7 & 3 & 0 & 0 & 2 & 3 \\
\hline Rainforest & BF4b & 2013 & 0 & 0 & 0 & 0 & 5 & 4 & 0 & 1 & 0 & 28 & 6 & 0 & 16 & 6 & 0 & 0 & 1 & 0 & 0 & 0 & 0 \\
\hline Rainforest & $\mathrm{HF} 1 \mathrm{~b}$ & 2013 & 0 & 0 & 6 & 0 & 0 & 6 & 9 & 0 & 0 & 15 & 18 & 0 & 0 & 16 & 14 & 0 & 0 & 11 & 0 & 0 & 5 \\
\hline Rainforest & HF2b & 2013 & 0 & 0 & 0 & 0 & 0 & 0 & 0 & 0 & 0 & 0 & 0 & 0 & 1 & 0 & 0 & 0 & 0 & 0 & 0 & 0 & 0 \\
\hline Rainforest & HF3b & 2013 & 0 & 0 & 0 & 0 & 0 & 0 & 0 & 0 & 0 & 3 & 8 & 1 & 2 & 21 & 18 & 0 & 0 & 3 & 0 & 0 & 0 \\
\hline Rainforest & HF4b & 2013 & 0 & 1 & 0 & 0 & 0 & 4 & 0 & 0 & 0 & 6 & 45 & 0 & 0 & 18 & 1 & 4 & 0 & 2 & 0 & 0 & 0 \\
\hline Rainforest & $\mathrm{BF} 1 \mathrm{~b}$ & 2016 & 0 & 0 & 8 & 0 & 12 & 0 & 0 & 4 & 0 & 16 & 0 & 0 & 0 & 0 & 64 & 8 & 0 & 0 & 0 & 4 & 0 \\
\hline Rainforest & $\mathrm{BF} 2 \mathrm{~b}$ & 2016 & 4 & 0 & 0 & 8 & 0 & 0 & 12 & 32 & 0 & 36 & 0 & 0 & 0 & 0 & 4 & 4 & 0 & 12 & 0 & 0 & 0 \\
\hline Rainforest & BF3b & 2016 & 0 & 0 & 0 & 0 & 0 & 0 & 16 & 0 & 0 & 0 & 0 & 0 & 4 & 0 & 0 & 0 & 0 & 0 & 0 & 0 & 0 \\
\hline Rainforest & HF1b & 2016 & 0 & 0 & 0 & 0 & 0 & 0 & 0 & 0 & 0 & 12 & 0 & 0 & 0 & 0 & 0 & 0 & 0 & 0 & 0 & 0 & 0 \\
\hline Rainforest & $\mathrm{HF} 2 \mathrm{~b}$ & 2016 & 0 & 0 & 0 & 0 & 12 & 0 & 0 & 0 & 0 & 8 & 0 & 0 & 0 & 0 & 16 & 0 & 0 & 0 & 4 & 12 & 16 \\
\hline Rainforest & HF3b & 2016 & 0 & 0 & 4 & 0 & 36 & 0 & 28 & 8 & 0 & 68 & 0 & 0 & 0 & 0 & 16 & 40 & 0 & 0 & 0 & 0 & 4 \\
\hline Rainforest & HFr1b & 2016 & 0 & 0 & 0 & 0 & 8 & 0 & 0 & 0 & 0 & 4 & 0 & 0 & 0 & 4 & 0 & 0 & 0 & 0 & 0 & 0 & 0 \\
\hline Rainforest & $\mathrm{HFr} 2 \mathrm{~b}$ & 2016 & 0 & 0 & 8 & 0 & 16 & 0 & 16 & 0 & 0 & 84 & 0 & 0 & 0 & 4 & 0 & 0 & 0 & 0 & 12 & 0 & 16 \\
\hline Rainforest & HFr3b & 2016 & 0 & 0 & 0 & 0 & 4 & 0 & 4 & 0 & 0 & 4 & 0 & 0 & 0 & 0 & 0 & 0 & 0 & 0 & 0 & 0 & 8 \\
\hline Rainforest & $\mathrm{HFr} 4 \mathrm{~b}$ & 2016 & 0 & 0 & 0 & 0 & 4 & 0 & 0 & 0 & 0 & 0 & 0 & 0 & 0 & 0 & 4 & 0 & 0 & 0 & 4 & 4 & 0 \\
\hline Jungle rubber & $\mathrm{BJ} 2 \mathrm{~b}$ & 2013 & 0 & 2 & 0 & 0 & 14 & 0 & 21 & 2 & 0 & 2 & 0 & 1 & 0 & 0 & 0 & 2 & 14 & 10 & 0 & 0 & 0 \\
\hline Jungle rubber & $\mathrm{BJ} 3 \mathrm{~b}$ & 2013 & 0 & 0 & 1 & 0 & 0 & 0 & 98 & 7 & 10 & 7 & 0 & 0 & 0 & 0 & 0 & 0 & 0 & 13 & 0 & 0 & 0 \\
\hline Jungle rubber & $\mathrm{BJ} 4 \mathrm{~b}$ & 2013 & 0 & 0 & 0 & 0 & 0 & 0 & 21 & 16 & 0 & 4 & 0 & 0 & 0 & 0 & 0 & 0 & 0 & 0 & 0 & 0 & 0 \\
\hline Jungle rubber & $\mathrm{BJ} 5 \mathrm{~b}$ & 2013 & 0 & 0 & 0 & 0 & 0 & 0 & 12 & 11 & 0 & 0 & 0 & 0 & 0 & 0 & 0 & 0 & 0 & 0 & 0 & 0 & 1 \\
\hline Jungle rubber & $\mathrm{HJ} 1 \mathrm{~b}$ & 2013 & 0 & 4 & 0 & 1 & 1 & 0 & 68 & 81 & 0 & 0 & 0 & 0 & 0 & 0 & 0 & 0 & 0 & 0 & 2 & 0 & 0 \\
\hline Jungle rubber & $\mathrm{HJ} 2 \mathrm{~b}$ & 2013 & 0 & 1 & 0 & 0 & 5 & 0 & 15 & 2 & 18 & 32 & 0 & 0 & 0 & 0 & 14 & 0 & 0 & 0 & 0 & 0 & 0 \\
\hline Jungle rubber & $\mathrm{HJ} 3 \mathrm{~b}$ & 2013 & 0 & 0 & 0 & 0 & 1 & 0 & 1 & 0 & 0 & 0 & 0 & 0 & 0 & 1 & 1 & 0 & 0 & 0 & 1 & 0 & 0 \\
\hline Jungle rubber & $\mathrm{HJ} 4 \mathrm{~b}$ & 2013 & 0 & 0 & 0 & 0 & 1 & 0 & 13 & 17 & 0 & 10 & 0 & 0 & 0 & 0 & 0 & 0 & 0 & 3 & 4 & 0 & 0 \\
\hline Oil palm & $\mathrm{BO} 2 \mathrm{~b}$ & 2013 & 0 & 0 & 0 & 0 & 0 & 0 & 0 & 0 & 0 & 0 & 2 & 0 & 4 & 0 & 0 & 0 & 1 & 0 & 0 & 0 & 0 \\
\hline Oil palm & $\mathrm{BO} 3 \mathrm{~b}$ & 2013 & 0 & 0 & 4 & 0 & 0 & 0 & 32 & 0 & 0 & 0 & 3 & 2 & 5 & 5 & 8 & 0 & 0 & 0 & 0 & 0 & 1 \\
\hline
\end{tabular}


Chapter 3

\begin{tabular}{|c|c|c|c|c|c|c|c|c|c|c|c|c|c|c|c|c|c|c|c|c|c|c|c|}
\hline Systems & Plot & Year & Acrl & Acr 2 & Allo & Call1 & Asco & Dicrl & Folce & Folspa & Homci & Isomi & Lep1 & Mega & Ony & Parl & Pseul & Ram & Sphal & Spha2 & Sphyl & Supl & Xen1 \\
\hline Oil palm & $\mathrm{BO} 4 \mathrm{~b}$ & 2013 & 3 & 0 & 0 & 0 & 0 & 0 & 0 & 0 & 0 & 6 & 3 & 0 & 0 & 0 & 0 & 0 & 0 & 1 & 0 & 0 & 0 \\
\hline Oil palm & $\mathrm{BO} 5 \mathrm{~b}$ & 2013 & 0 & 0 & 0 & 0 & 0 & 0 & 12 & 3 & 0 & 3 & 0 & 0 & 3 & 0 & 5 & 0 & 0 & 0 & 0 & 0 & 0 \\
\hline Oil palm & $\mathrm{HO} 1 \mathrm{~b}$ & 2013 & 0 & 2 & 0 & 0 & 0 & 0 & 25 & 0 & 0 & 0 & 0 & 0 & 0 & 0 & 3 & 0 & 0 & 0 & 0 & 0 & 0 \\
\hline Oil palm & $\mathrm{HO} 2 b$ & 2013 & 0 & 0 & 0 & 0 & 0 & 0 & 0 & 0 & 0 & 10 & 1 & 0 & 0 & 0 & 0 & 0 & 0 & 0 & 0 & 0 & 0 \\
\hline Oil palm & $\mathrm{HO} 3 \mathrm{~b}$ & 2013 & 0 & 0 & 0 & 0 & 0 & 0 & 0 & 0 & 0 & 13 & 1 & 0 & 1 & 0 & 0 & 0 & 0 & 0 & 0 & 0 & 1 \\
\hline Oil palm & $\mathrm{BO} 1 \mathrm{~b}$ & 2016 & 8 & 0 & 4 & 0 & 0 & 4 & 12 & 8 & 0 & 52 & 0 & 0 & 0 & 0 & 0 & 192 & 0 & 8 & 0 & 0 & 0 \\
\hline Oil palm & $\mathrm{BO} 2 \mathrm{~b}$ & 2016 & 0 & 0 & 0 & 0 & 0 & 0 & 4 & 0 & 0 & 0 & 0 & 0 & 0 & 0 & 0 & 4 & 0 & 0 & 0 & 0 & 0 \\
\hline Oil palm & $\mathrm{BO} 3 \mathrm{~b}$ & 2016 & 4 & 0 & 0 & 0 & 0 & 0 & 12 & 12 & 0 & 0 & 0 & 0 & 0 & 0 & 0 & 0 & 0 & 0 & 0 & 0 & 0 \\
\hline Oil palm & $\mathrm{BO} 4 \mathrm{~b}$ & 2016 & 0 & 0 & 0 & 0 & 0 & 0 & 48 & 0 & 0 & 0 & 0 & 0 & 0 & 0 & 4 & 0 & 0 & 0 & 0 & 0 & 0 \\
\hline Oil palm & $\mathrm{HO} 1 \mathrm{~b}$ & 2016 & 0 & 0 & 0 & 0 & 0 & 0 & 0 & 0 & 0 & 0 & 0 & 0 & 0 & 0 & 0 & 0 & 0 & 0 & 0 & 0 & 0 \\
\hline Oil palm & $\mathrm{HO} 2 \mathrm{~b}$ & 2016 & 0 & 0 & 0 & 0 & 0 & 0 & 16 & 0 & 0 & 0 & 0 & 0 & 8 & 0 & 0 & 0 & 0 & 0 & 0 & 0 & 0 \\
\hline Oil palm & $\mathrm{HO} 3 \mathrm{~b}$ & 2016 & 0 & 0 & 0 & 0 & 0 & 0 & 4 & 0 & 0 & 0 & 0 & 0 & 0 & 0 & 0 & 0 & 0 & 0 & 0 & 0 & 0 \\
\hline Oil palm & $\mathrm{HO} 4 \mathrm{~b}$ & 2016 & 0 & 0 & 0 & 0 & 0 & 0 & 4 & 0 & 0 & 0 & 0 & 0 & 0 & 0 & 0 & 8 & 0 & 0 & 0 & 0 & 0 \\
\hline Oil palm & $\mathrm{HO} 4 \mathrm{rb}$ & 2016 & 0 & 0 & 0 & 0 & 0 & 0 & 0 & 0 & 0 & 20 & 0 & 0 & 0 & 0 & 0 & 0 & 0 & 0 & 0 & 0 & 0 \\
\hline Oil palm & HOr2b & 2016 & 0 & 0 & 0 & 0 & 0 & 0 & 12 & 0 & 0 & 0 & 0 & 0 & 0 & 0 & 0 & 0 & 0 & 0 & 0 & 0 & 0 \\
\hline Oil palm & HOr3b & 2016 & 0 & 0 & 0 & 0 & 0 & 0 & 20 & 4 & 0 & 0 & 0 & 0 & 0 & 0 & 0 & 0 & 0 & 0 & 0 & 0 & 0 \\
\hline Rubber & BR1b & 2013 & 0 & 0 & 0 & 0 & 16 & 0 & 12 & 2 & 1 & 8 & 0 & 5 & 0 & 0 & 6 & 5 & 0 & 0 & 0 & 0 & 0 \\
\hline Rubber & $\mathrm{BR} 2 \mathrm{~b}$ & 2013 & 3 & 0 & 0 & 0 & 6 & 0 & 7 & 0 & 0 & 0 & 0 & 0 & 0 & 0 & 0 & 0 & 0 & 0 & 3 & 0 & 0 \\
\hline Rubber & BR3b & 2013 & 0 & 0 & 0 & 0 & 18 & 0 & 9 & 8 & 13 & 5 & 3 & 0 & 0 & 2 & 5 & 11 & 0 & 0 & 0 & 0 & 0 \\
\hline Rubber & HR1b & 2013 & 0 & 0 & 0 & 0 & 25 & 0 & 46 & 0 & 1 & 18 & 0 & 4 & 0 & 0 & 4 & 4 & 0 & 0 & 0 & 0 & 0 \\
\hline Rubber & $\mathrm{HR} 2 \mathrm{~b}$ & 2013 & 2 & 0 & 0 & 0 & 14 & 0 & 20 & 0 & 13 & 0 & 0 & 7 & 0 & 1 & 0 & 0 & 0 & 4 & 0 & 0 & 0 \\
\hline Rubber & HR3b & 2013 & 0 & 0 & 1 & 0 & 0 & 0 & 8 & 1 & 1 & 0 & 0 & 0 & 0 & 0 & 3 & 0 & 0 & 0 & 0 & 0 & 0 \\
\hline Rubber & $\mathrm{HR} 4 \mathrm{~b}$ & 2013 & 0 & 0 & 0 & 0 & 2 & 0 & 2 & 4 & 0 & 0 & 0 & 0 & 0 & 0 & 1 & 0 & 0 & 0 & 0 & 0 & 0 \\
\hline Rubber & BR1b & 2016 & 0 & 0 & 0 & 0 & 8 & 0 & 4 & 0 & 0 & 0 & 0 & 0 & 0 & 0 & 0 & 0 & 0 & 0 & 0 & 0 & 0 \\
\hline Rubber & $\mathrm{BR} 2 \mathrm{~b}$ & 2016 & 0 & 0 & 0 & 0 & 0 & 0 & 40 & 32 & 0 & 0 & 0 & 0 & 0 & 4 & 0 & 0 & 0 & 0 & 0 & 0 & 0 \\
\hline Rubber & BR3b & 2016 & 0 & 0 & 0 & 0 & 8 & 0 & 32 & 8 & 4 & 0 & 0 & 0 & 0 & 0 & 0 & 0 & 0 & 0 & 0 & 0 & 12 \\
\hline Rubber & $\mathrm{BR} 4 \mathrm{~b}$ & 2016 & 4 & 0 & 0 & 0 & 0 & 0 & 0 & 0 & 8 & 0 & 0 & 0 & 0 & 0 & 4 & 4 & 0 & 0 & 0 & 0 & 0 \\
\hline Rubber & $\mathrm{HR} 2 \mathrm{~b}$ & 2016 & 0 & 0 & 0 & 0 & 0 & 0 & 0 & 0 & 8 & 0 & 0 & 0 & 0 & 0 & 0 & 0 & 0 & 0 & 0 & 0 & 0 \\
\hline Rubber & HR3b & 2016 & 0 & 0 & 4 & 0 & 8 & 0 & 0 & 0 & 0 & 8 & 0 & 0 & 0 & 0 & 0 & 0 & 0 & 0 & 0 & 0 & 0 \\
\hline Rubber & $\mathrm{HR} 4 \mathrm{~b}$ & 2016 & 0 & 0 & 0 & 0 & 4 & 0 & 4 & 0 & 4 & 4 & 0 & 0 & 0 & 0 & 0 & 0 & 0 & 0 & 0 & 0 & 0 \\
\hline
\end{tabular}


Chapter 3

\begin{tabular}{|c|c|c|c|c|c|c|c|c|c|c|c|c|c|c|c|c|c|c|c|c|c|c|c|}
\hline Systems & Plot & Year & Acrl & Acr 2 & Allo & Call1 & Asco & Dicrl & Folce & Folspa & Homci & Isomi & Lep1 & Mega & Ony & Parl & Pseul & Ram & Sphal & Spha2 & Sphyl & Supl & Xenl \\
\hline Rubber & $\mathrm{HRr} 2 \mathrm{~b}$ & 2016 & 0 & 0 & 0 & 0 & 12 & 0 & 12 & 0 & 0 & 8 & 0 & 0 & 0 & 0 & 0 & 0 & 0 & 0 & 0 & 0 & 8 \\
\hline Rubber & $\mathrm{HRr} 3 \mathrm{~b}$ & 2016 & 0 & 0 & 0 & 0 & 12 & 0 & 0 & 0 & 8 & 0 & 0 & 0 & 0 & 0 & 0 & 0 & 0 & 0 & 0 & 0 & 0 \\
\hline Rubber & $\mathrm{HRr} 4 \mathrm{~b}$ & 2016 & 0 & 0 & 0 & 0 & 16 & 0 & 8 & 12 & 0 & 0 & 0 & 0 & 0 & 0 & 0 & 0 & 0 & 0 & 0 & 0 & 0 \\
\hline
\end{tabular}

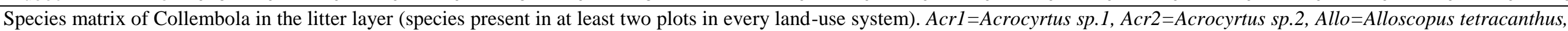

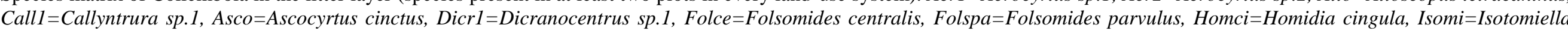

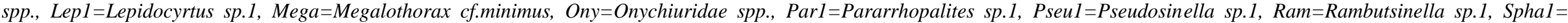
Sphaeridia sp.1, Spha2=Sphaeridia sp.2, Sphyl=Sphyroteca sp.1, Sup1=Superodontella sp.1, Xen1=Xenylla sp.1 
Chapter 3

Supplementary Table S3.3. Species matrix of Collembola (individuals per plot) in the soil layer

\begin{tabular}{|c|c|c|c|c|c|c|c|c|c|c|c|c|c|c|c|c|c|c|c|c|c|c|c|}
\hline Systems & Plot & Year & Acrl & Acr 3 & Allo & Asco & Cyps 1 & Cyphl & Dicrl & Folsce & Folspa & Folny & Homci & Isomi & Isodel & Isotus & Lep1 & Mega & Ony & Pseul & Ram & Sphyl & Xen1 \\
\hline Rainforest & BF1b & 2013 & 2 & 0 & 0 & 0 & 10 & 0 & 0 & 0 & 0 & 0 & 0 & 5 & 0 & 0 & 2 & 0 & 0 & 12 & 0 & 0 & 0 \\
\hline Rainforest & $\mathrm{BF} 2 \mathrm{~b}$ & 2013 & 0 & 3 & 1 & 1 & 0 & 0 & 0 & 8 & 10 & 0 & 0 & 17 & 0 & 0 & 1 & 0 & 15 & 6 & 0 & 1 & 0 \\
\hline Rainforest & $\mathrm{BF} 3 \mathrm{~b}$ & 2013 & 0 & 0 & 0 & 0 & 2 & 0 & 0 & 0 & 0 & 1 & 0 & 0 & 0 & 0 & 0 & 0 & 0 & 0 & 0 & 0 & 0 \\
\hline Rainforest & $\mathrm{BF} 4 \mathrm{~b}$ & 2013 & 0 & 2 & 0 & 0 & 7 & 0 & 0 & 1 & 0 & 0 & 0 & 385 & 0 & 0 & 0 & 3 & 93 & 54 & 2 & 0 & 1 \\
\hline Rainforest & HF1b & 2013 & 0 & 0 & 0 & 0 & 0 & 0 & 0 & 1 & 0 & 0 & 1 & 34 & 0 & 0 & 4 & 0 & 0 & 39 & 0 & 0 & 1 \\
\hline Rainforest & $\mathrm{HF} 2 \mathrm{~b}$ & 2013 & 0 & 0 & 0 & 0 & 0 & 0 & 1 & 0 & 0 & 0 & 0 & 41 & 0 & 0 & 6 & 0 & 0 & 39 & 0 & 0 & 2 \\
\hline Rainforest & $\mathrm{HF} 3 \mathrm{~b}$ & 2013 & 0 & 0 & 0 & 0 & 0 & 0 & 0 & 0 & 0 & 0 & 0 & 0 & 0 & 0 & 11 & 0 & 0 & 28 & 1 & 0 & 0 \\
\hline Rainforest & $\mathrm{HF} 4 \mathrm{~b}$ & 2013 & 0 & 0 & 0 & 0 & 0 & 0 & 0 & 0 & 0 & 0 & 0 & 5 & 0 & 0 & 7 & 0 & 0 & 56 & 16 & 0 & 8 \\
\hline Rainforest & BF1b & 2016 & 0 & 0 & 0 & 0 & 0 & 0 & 0 & 0 & 0 & 0 & 0 & 4 & 0 & 0 & 0 & 0 & 0 & 4 & 0 & 0 & 0 \\
\hline Rainforest & $\mathrm{BF} 2 \mathrm{~b}$ & 2016 & 0 & 0 & 16 & 0 & 0 & 0 & 0 & 4 & 0 & 0 & 0 & 12 & 0 & 0 & 0 & 0 & 0 & 0 & 8 & 0 & 12 \\
\hline Rainforest & $\mathrm{BF} 3 \mathrm{~b}$ & 2016 & 0 & 0 & 4 & 4 & 0 & 0 & 0 & 0 & 0 & 0 & 0 & 4 & 0 & 0 & 0 & 0 & 0 & 8 & 0 & 0 & 0 \\
\hline Rainforest & $\mathrm{BF} 4 \mathrm{~b}$ & 2016 & 0 & 0 & 0 & 0 & 0 & 0 & 0 & 0 & 0 & 0 & 0 & 4 & 0 & 0 & 0 & 0 & 12 & 8 & 0 & 0 & 0 \\
\hline Rainforest & HF1b & 2016 & 0 & 0 & 12 & 0 & 0 & 0 & 0 & 0 & 0 & 0 & 0 & 4 & 0 & 4 & 0 & 0 & 0 & 24 & 0 & 0 & 0 \\
\hline Rainforest & $\mathrm{HF} 2 \mathrm{~b}$ & 2016 & 0 & 0 & 12 & 4 & 0 & 0 & 0 & 0 & 0 & 0 & 0 & 0 & 0 & 0 & 0 & 0 & 0 & 0 & 0 & 0 & 0 \\
\hline Rainforest & $\mathrm{HF} 4 \mathrm{~b}$ & 2016 & 0 & 0 & 8 & 32 & 4 & 4 & 0 & 0 & 0 & 0 & 0 & 88 & 0 & 0 & 0 & 0 & 16 & 8 & 16 & 12 & 12 \\
\hline Rainforest & HFr1b & 2016 & 0 & 0 & 0 & 0 & 0 & 0 & 0 & 0 & 0 & 0 & 4 & 0 & 0 & 0 & 0 & 0 & 0 & 0 & 0 & 0 & 0 \\
\hline Rainforest & $\mathrm{HFr} 2 \mathrm{~b}$ & 2016 & 0 & 0 & 4 & 8 & 16 & 0 & 0 & 0 & 0 & 0 & 0 & 4 & 0 & 0 & 0 & 0 & 0 & 4 & 0 & 0 & 0 \\
\hline Rainforest & HFr3b & 2016 & 0 & 0 & 0 & 8 & 0 & 0 & 0 & 4 & 0 & 0 & 0 & 0 & 0 & 0 & 0 & 0 & 0 & 0 & 0 & 0 & 0 \\
\hline Rainforest & $\mathrm{HFr} 4 \mathrm{~b}$ & 2016 & 0 & 0 & 0 & 4 & 0 & 0 & 0 & 0 & 0 & 0 & 0 & 8 & 0 & 0 & 0 & 0 & 0 & 0 & 0 & 0 & 0 \\
\hline Jungle rubber & $\mathrm{BJ} 2 \mathrm{~b}$ & 2013 & 0 & 0 & 2 & 0 & 0 & 2 & 0 & 11 & 1 & 0 & 1 & 2 & 0 & 0 & 0 & 0 & 0 & 20 & 3 & 0 & 0 \\
\hline Jungle rubber & $\mathrm{BJ} 3 \mathrm{~b}$ & 2013 & 0 & 0 & 0 & 8 & 0 & 1 & 0 & 12 & 1 & 0 & 3 & 12 & 0 & 0 & 0 & 0 & 0 & 16 & 2 & 0 & 0 \\
\hline Jungle rubber & $\mathrm{BJ} 4 \mathrm{~b}$ & 2013 & 0 & 0 & 0 & 0 & 0 & 0 & 0 & 32 & 4 & 2 & 0 & 0 & 0 & 0 & 0 & 0 & 0 & 0 & 0 & 0 & 0 \\
\hline Jungle rubber & BJ5b & 2013 & 0 & 0 & 1 & 3 & 0 & 0 & 0 & 3 & 0 & 0 & 1 & 1 & 0 & 0 & 0 & 1 & 0 & 26 & 25 & 1 & 1 \\
\hline Jungle rubber & $\mathrm{HJ} 1 \mathrm{~b}$ & 2013 & 0 & 0 & 0 & 3 & 0 & 0 & 0 & 12 & 5 & 0 & 0 & 6 & 0 & 0 & 0 & 0 & 0 & 0 & 2 & 0 & 0 \\
\hline Jungle rubber & $\mathrm{HJ} 3 \mathrm{~b}$ & 2013 & 0 & 0 & 0 & 1 & 0 & 0 & 0 & 0 & 0 & 0 & 0 & 2 & 6 & 0 & 0 & 0 & 0 & 3 & 0 & 0 & 0 \\
\hline Jungle rubber & $\mathrm{HJ} 4 \mathrm{~b}$ & 2013 & 0 & 0 & 0 & 1 & 0 & 0 & 0 & 0 & 2 & 1 & 0 & 0 & 0 & 0 & 0 & 0 & 6 & 7 & 0 & 0 & 0 \\
\hline Oil palm & $\mathrm{BO} 2 \mathrm{~b}$ & 2013 & 0 & 0 & 0 & 0 & 0 & 1 & 0 & 13 & 0 & 0 & 0 & 7 & 0 & 0 & 2 & 0 & 0 & 47 & 0 & 0 & 0 \\
\hline
\end{tabular}


Chapter 3

\begin{tabular}{|c|c|c|c|c|c|c|c|c|c|c|c|c|c|c|c|c|c|c|c|c|c|c|c|}
\hline Systems & Plot & Year & Acrl & Acr3 & Allo & Asco & Cyps 1 & Cyph1 & Dicrl & Folsce & Folspa & Folny & Homci & Isomi & Isode1 & Isotus & Lep1 & Mega & Ony & Pseul & Ram & Sphyl & Xen1 \\
\hline Oil palm & $\mathrm{BO} 3 \mathrm{~b}$ & 2013 & 0 & 0 & 0 & 2 & 0 & 0 & 0 & 5 & 0 & 0 & 7 & 14 & 0 & 0 & 0 & 1 & 16 & 14 & 4 & 0 & 0 \\
\hline Oil palm & $\mathrm{BO} 4 \mathrm{~b}$ & 2013 & 0 & 0 & 9 & 0 & 0 & 0 & 0 & 57 & 0 & 0 & 0 & 91 & 0 & 2 & 2 & 1 & 5 & 38 & 1 & 0 & 0 \\
\hline Oil palm & HO1b & 2013 & 0 & 5 & 20 & 0 & 0 & 0 & 0 & 21 & 0 & 0 & 0 & 37 & 0 & 0 & 7 & 0 & 0 & 41 & 0 & 0 & 0 \\
\hline Oil palm & $\mathrm{HO} 2 \mathrm{~b}$ & 2013 & 0 & 2 & 0 & 0 & 0 & 0 & 13 & 23 & 0 & 0 & 0 & 21 & 0 & 0 & 1 & 0 & 1 & 11 & 0 & 0 & 0 \\
\hline Oil palm & $\mathrm{HO} 3 \mathrm{~b}$ & 2013 & 0 & 0 & 0 & 0 & 0 & 0 & 0 & 0 & 0 & 0 & 0 & 11 & 0 & 0 & 1 & 0 & 0 & 10 & 0 & 0 & 0 \\
\hline Oil palm & BO1b & 2016 & 4 & 0 & 16 & 0 & 0 & 0 & 0 & 8 & 0 & 0 & 0 & 0 & 0 & 0 & 0 & 0 & 0 & 4 & 36 & 0 & 0 \\
\hline Oil palm & $\mathrm{BO} 2 \mathrm{~b}$ & 2016 & 16 & 0 & 12 & 0 & 0 & 0 & 0 & 16 & 0 & 0 & 0 & 8 & 8 & 0 & 0 & 0 & 0 & 4 & 12 & 0 & 0 \\
\hline Oil palm & $\mathrm{BO} 3 \mathrm{~b}$ & 2016 & 0 & 0 & 4 & 12 & 0 & 0 & 0 & 8 & 0 & 0 & 0 & 0 & 0 & 0 & 0 & 0 & 0 & 8 & 0 & 0 & 0 \\
\hline Oil palm & $\mathrm{BO} 4 \mathrm{~b}$ & 2016 & 0 & 0 & 16 & 0 & 0 & 0 & 12 & 12 & 0 & 0 & 0 & 0 & 12 & 0 & 0 & 0 & 0 & 28 & 0 & 0 & 0 \\
\hline Oil palm & $\mathrm{HO} 2 \mathrm{~b}$ & 2016 & 0 & 0 & 0 & 4 & 0 & 0 & 0 & 8 & 0 & 0 & 0 & 0 & 0 & 8 & 0 & 0 & 0 & 0 & 4 & 0 & 0 \\
\hline Oil palm & $\mathrm{HO} 3 \mathrm{~b}$ & 2016 & 4 & 0 & 0 & 0 & 0 & 0 & 0 & 8 & 0 & 20 & 4 & 4 & 4 & 0 & 0 & 0 & 8 & 0 & 0 & 0 & 0 \\
\hline Oil palm & $\mathrm{HO} 4 \mathrm{~b}$ & 2016 & 0 & 0 & 16 & 0 & 4 & 0 & 0 & 4 & 0 & 16 & 0 & 0 & 0 & 0 & 0 & 0 & 4 & 0 & 4 & 0 & 0 \\
\hline Oil palm & $\mathrm{HO} 4 \mathrm{rb}$ & 2016 & 0 & 0 & 0 & 4 & 0 & 0 & 0 & 8 & 4 & 0 & 0 & 60 & 0 & 0 & 0 & 0 & 12 & 32 & 64 & 0 & 0 \\
\hline Oil palm & HOr1b & 2016 & 0 & 0 & 24 & 0 & 0 & 0 & 0 & 16 & 0 & 0 & 0 & 4 & 0 & 0 & 0 & 0 & 0 & 0 & 0 & 0 & 0 \\
\hline Rubber & BR1b & 2013 & 0 & 0 & 6 & 22 & 1 & 0 & 0 & 5 & 1 & 0 & 0 & 22 & 0 & 0 & 2 & 2 & 0 & 2 & 4 & 0 & 0 \\
\hline Rubber & $\mathrm{BR} 2 \mathrm{~b}$ & 2013 & 3 & 1 & 0 & 0 & 0 & 0 & 0 & 16 & 5 & 0 & 0 & 3 & 0 & 0 & 0 & 2 & 0 & 14 & 0 & 0 & 0 \\
\hline Rubber & $\mathrm{BR} 3 \mathrm{~b}$ & 2013 & 2 & 0 & 0 & 0 & 0 & 0 & 0 & 7 & 0 & 0 & 1 & 0 & 0 & 0 & 0 & 0 & 0 & 15 & 25 & 0 & 0 \\
\hline Rubber & $\mathrm{BR} 4 \mathrm{~b}$ & 2013 & 0 & 0 & 0 & 0 & 0 & 0 & 0 & 4 & 0 & 0 & 0 & 0 & 1 & 0 & 0 & 0 & 0 & 8 & 0 & 0 & 0 \\
\hline Rubber & HR1b & 2013 & 0 & 4 & 17 & 6 & 0 & 1 & 0 & 7 & 0 & 0 & 0 & 20 & 0 & 0 & 0 & 0 & 0 & 35 & 0 & 2 & 0 \\
\hline Rubber & $\mathrm{HR} 2 \mathrm{~b}$ & 2013 & 0 & 0 & 5 & 18 & 5 & 0 & 0 & 2 & 0 & 0 & 0 & 0 & 0 & 0 & 0 & 6 & 0 & 0 & 0 & 0 & 0 \\
\hline Rubber & HR3b & 2013 & 2 & 0 & 6 & 0 & 0 & 0 & 0 & 6 & 4 & 0 & 0 & 0 & 0 & 0 & 0 & 0 & 0 & 15 & 0 & 0 & 0 \\
\hline Rubber & $\mathrm{HR} 4 \mathrm{~b}$ & 2013 & 0 & 0 & 2 & 0 & 0 & 0 & 0 & 0 & 0 & 0 & 0 & 0 & 1 & 0 & 0 & 0 & 0 & 20 & 0 & 0 & 0 \\
\hline Rubber & BR1b & 2016 & 0 & 0 & 0 & 8 & 0 & 0 & 0 & 0 & 0 & 0 & 0 & 8 & 0 & 0 & 0 & 0 & 0 & 0 & 4 & 0 & 0 \\
\hline Rubber & $\mathrm{BR} 2 \mathrm{~b}$ & 2016 & 0 & 0 & 28 & 0 & 0 & 0 & 0 & 16 & 4 & 0 & 0 & 0 & 0 & 0 & 0 & 0 & 0 & 24 & 0 & 0 & 0 \\
\hline Rubber & BR3b & 2016 & 0 & 0 & 0 & 0 & 0 & 0 & 0 & 16 & 0 & 0 & 0 & 0 & 0 & 0 & 0 & 0 & 0 & 4 & 0 & 0 & 0 \\
\hline Rubber & $\mathrm{BR} 4 \mathrm{~b}$ & 2016 & 0 & 0 & 20 & 0 & 0 & 0 & 0 & 0 & 0 & 0 & 0 & 0 & 0 & 0 & 0 & 0 & 0 & 4 & 32 & 0 & 0 \\
\hline Rubber & HR1b & 2016 & 0 & 0 & 0 & 12 & 0 & 0 & 0 & 4 & 0 & 0 & 0 & 0 & 0 & 0 & 0 & 0 & 0 & 40 & 0 & 0 & 0 \\
\hline Rubber & $\mathrm{HR} 2 \mathrm{~b}$ & 2016 & 0 & 0 & 36 & 44 & 0 & 0 & 0 & 12 & 0 & 0 & 0 & 0 & 0 & 0 & 0 & 0 & 0 & 0 & 4 & 0 & 0 \\
\hline
\end{tabular}


Chapter 3

\begin{tabular}{|c|c|c|c|c|c|c|c|c|c|c|c|c|c|c|c|c|c|c|c|c|c|c|c|}
\hline Systems & Plot & Year & Acrl & Acr3 & Allo & Asco & Cyps1 & Cyph1 & Dicrl & Folsce & Folspa & Folny & Homci & Isomi & Isodel & Isotus & Lep 1 & Mega & Ony & Pseul & Ram & Sphyl & Xen1 \\
\hline Rubber & $\mathrm{HR} 4 \mathrm{~b}$ & 2016 & 0 & 0 & 0 & 12 & 0 & 8 & 0 & 0 & 0 & 0 & 0 & 0 & 0 & 0 & 0 & 0 & 0 & 0 & 4 & 0 & 0 \\
\hline Rubber & HRr1b & 2016 & 0 & 0 & 12 & 8 & 0 & 0 & 0 & 4 & 0 & 0 & 8 & 0 & 0 & 8 & 0 & 0 & 0 & 0 & 0 & 0 & 0 \\
\hline Rubber & HRr2b & 2016 & 0 & 0 & 4 & 8 & 0 & 0 & 0 & 0 & 0 & 0 & 0 & 0 & 0 & 0 & 0 & 0 & 0 & 0 & 0 & 0 & 0 \\
\hline Rubber & HRr3b & 2016 & 0 & 0 & 4 & 12 & 0 & 4 & 0 & 12 & 0 & 0 & 12 & 0 & 0 & 0 & 0 & 0 & 0 & 20 & 0 & 0 & 0 \\
\hline Rubber & $\mathrm{HRr} 4 \mathrm{~b}$ & 2016 & 0 & 0 & 0 & 16 & 0 & 0 & 0 & 4 & 8 & 4 & 12 & 8 & 0 & 0 & 0 & 0 & 0 & 0 & 0 & 0 & 0 \\
\hline
\end{tabular}

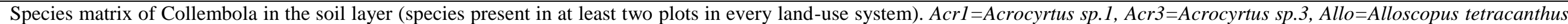

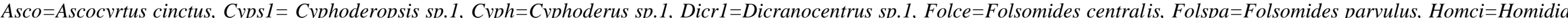

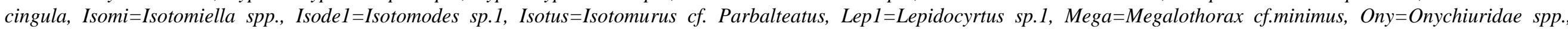
Pseul=Pseudosinella sp.1, Ram=Rambutsinella sp.1, Sphyl= Sphyroteca sp.1, Xen1= Xenylla sp.1 
Supplementary Table S3.4. Trait matrix of Collembola in litter layer based on density

\begin{tabular}{|c|c|c|c|c|c|c|c|c|c|c|c|c|c|c|c|c|c|c|c|c|}
\hline System & Plot & Year & $\mathrm{Ea}$ & Ms & PAOp & $\mathrm{Sp}$ & $\mathrm{Ae}$ & As & Af & Fs & $\mathrm{Fa}$ & $\mathrm{Fl}$ & $\mathrm{Pd}$ & $\mathrm{Pp}$ & $\mathrm{Pa}$ & $\mathrm{Pi}$ & Am & Ss & Ms & Ls \\
\hline Rainforest & $\mathrm{BF} 1 \mathrm{~b}$ & 2013 & 1 & 1 & 22 & 37 & 28 & 6 & 21 & 26 & 2 & 58 & 4 & 37 & 44 & 1 & 18 & 50 & 22 & 14 \\
\hline Rainforest & $\mathrm{BF} 2 \mathrm{~b}$ & 2013 & 28 & 0 & 51 & 0 & 0 & 1 & 2 & 22 & 30 & 2 & 21 & 0 & 32 & 1 & 0 & 54 & 0 & 0 \\
\hline Rainforest & $\mathrm{BF} 3 \mathrm{~b}$ & 2013 & 5 & 2 & 18 & 43 & 50 & 10 & 52 & 70 & 13 & 60 & 21 & 36 & 81 & 5 & 17 & 39 & 32 & 72 \\
\hline Rainforest & $\mathrm{BF} 4 \mathrm{~b}$ & 2013 & 1 & 0 & 18 & 15 & 11 & 14 & 28 & 15 & 17 & 43 & 6 & 22 & 46 & 1 & 17 & 59 & 16 & 0 \\
\hline Rainforest & HF1b & 2013 & 5 & 0 & 15 & 44 & 32 & 37 & 15 & 51 & 0 & 59 & 32 & 32 & 35 & 11 & 38 & 66 & 38 & 6 \\
\hline Rainforest & $\mathrm{HF} 2 \mathrm{~b}$ & 2013 & 0 & 0 & 1 & 0 & 0 & 0 & 0 & 0 & 1 & 0 & 0 & 0 & 1 & 0 & 0 & 1 & 0 & 0 \\
\hline Rainforest & $\mathrm{HF} 4 \mathrm{~b}$ & 2013 & 2 & 2 & 2 & 57 & 53 & 28 & 6 & 22 & 0 & 71 & 57 & 25 & 7 & 4 & 24 & 34 & 56 & 3 \\
\hline Rainforest & $\mathrm{BF} 1 \mathrm{~b}$ & 2016 & 4 & 4 & 16 & 104 & 96 & 0 & 16 & 8 & 0 & 120 & 8 & 24 & 92 & 4 & 20 & 24 & 96 & 8 \\
\hline Rainforest & $\mathrm{BF} 2 \mathrm{~b}$ & 2016 & 0 & 0 & 44 & 20 & 20 & 12 & 36 & 56 & 0 & 56 & 16 & 8 & 76 & 12 & 8 & 92 & 12 & 8 \\
\hline Rainforest & $\mathrm{BF} 3 \mathrm{~b}$ & 2016 & 4 & 0 & 20 & 0 & 0 & 0 & 0 & 16 & 4 & 0 & 16 & 0 & 4 & 0 & 0 & 20 & 0 & 0 \\
\hline Rainforest & HF1b & 2016 & 0 & 0 & 0 & 0 & 0 & 0 & 12 & 0 & 0 & 12 & 0 & 0 & 12 & 0 & 0 & 12 & 0 & 0 \\
\hline Rainforest & $\mathrm{HF} 2 \mathrm{~b}$ & 2016 & 28 & 12 & 12 & 36 & 36 & 4 & 8 & 40 & 0 & 36 & 16 & 16 & 32 & 12 & 4 & 40 & 36 & 0 \\
\hline Rainforest & HFr1b & 2016 & 0 & 0 & 0 & 8 & 8 & 4 & 4 & 4 & 0 & 12 & 0 & 12 & 4 & 0 & 4 & 8 & 8 & 0 \\
\hline Rainforest & $\mathrm{HFr} 2 \mathrm{~b}$ & 2016 & 16 & 0 & 24 & 32 & 24 & 20 & 84 & 52 & 0 & 116 & 32 & 36 & 100 & 0 & 28 & 132 & 28 & 8 \\
\hline Rainforest & HFr3b & 2016 & 8 & 0 & 4 & 4 & 4 & 0 & 4 & 12 & 0 & 8 & 12 & 4 & 4 & 0 & 0 & 16 & 4 & 0 \\
\hline Rainforest & $\mathrm{HFr} 4 \mathrm{~b}$ & 2016 & 4 & 4 & 4 & 8 & 8 & 4 & 0 & 8 & 0 & 8 & 0 & 8 & 4 & 4 & 4 & 8 & 8 & 0 \\
\hline Jungle rubber & $\mathrm{BJ} 2 \mathrm{~b}$ & 2013 & 0 & 0 & 23 & 18 & 18 & 27 & 2 & 50 & 0 & 20 & 23 & 18 & 5 & 24 & 2 & 50 & 18 & 2 \\
\hline Jungle rubber & $\mathrm{BJ} 3 \mathrm{~b}$ & 2013 & 0 & 0 & 106 & 1 & 10 & 13 & 7 & 118 & 0 & 18 & 98 & 10 & 15 & 13 & 1 & 125 & 0 & 11 \\
\hline Jungle rubber & $\mathrm{BJ} 4 \mathrm{~b}$ & 2013 & 0 & 0 & 37 & 0 & 0 & 0 & 4 & 37 & 0 & 4 & 21 & 0 & 20 & 0 & 0 & 41 & 0 & 0 \\
\hline Jungle rubber & $\mathrm{BJ} 5 \mathrm{~b}$ & 2013 & 1 & 0 & 23 & 0 & 0 & 0 & 1 & 25 & 0 & 0 & 13 & 0 & 12 & 0 & 0 & 24 & 0 & 1 \\
\hline Jungle rubber & $\mathrm{HJ} 1 \mathrm{~b}$ & 2013 & 0 & 0 & 149 & 15 & 15 & 5 & 3 & 151 & 0 & 21 & 71 & 17 & 84 & 0 & 3 & 158 & 9 & 5 \\
\hline Jungle rubber & $\mathrm{HJ} 2 \mathrm{~b}$ & 2013 & 0 & 0 & 19 & 21 & 39 & 0 & 32 & 20 & 0 & 70 & 15 & 24 & 51 & 0 & 0 & 51 & 20 & 19 \\
\hline Jungle rubber & HJ3b & 2013 & 0 & 0 & 1 & 2 & 2 & 2 & 0 & 3 & 0 & 2 & 1 & 3 & 1 & 0 & 2 & 3 & 2 & 0 \\
\hline Jungle rubber & HJ4b & 2013 & 0 & 0 & 31 & 1 & 1 & 7 & 10 & 38 & 0 & 11 & 13 & 5 & 28 & 3 & 4 & 48 & 1 & 0 \\
\hline Oil palm & $\mathrm{BO} 2 \mathrm{~b}$ & 2013 & 3 & 0 & 4 & 2 & 2 & 1 & 0 & 1 & 4 & 2 & 2 & 0 & 4 & 1 & 0 & 5 & 2 & 0 \\
\hline
\end{tabular}




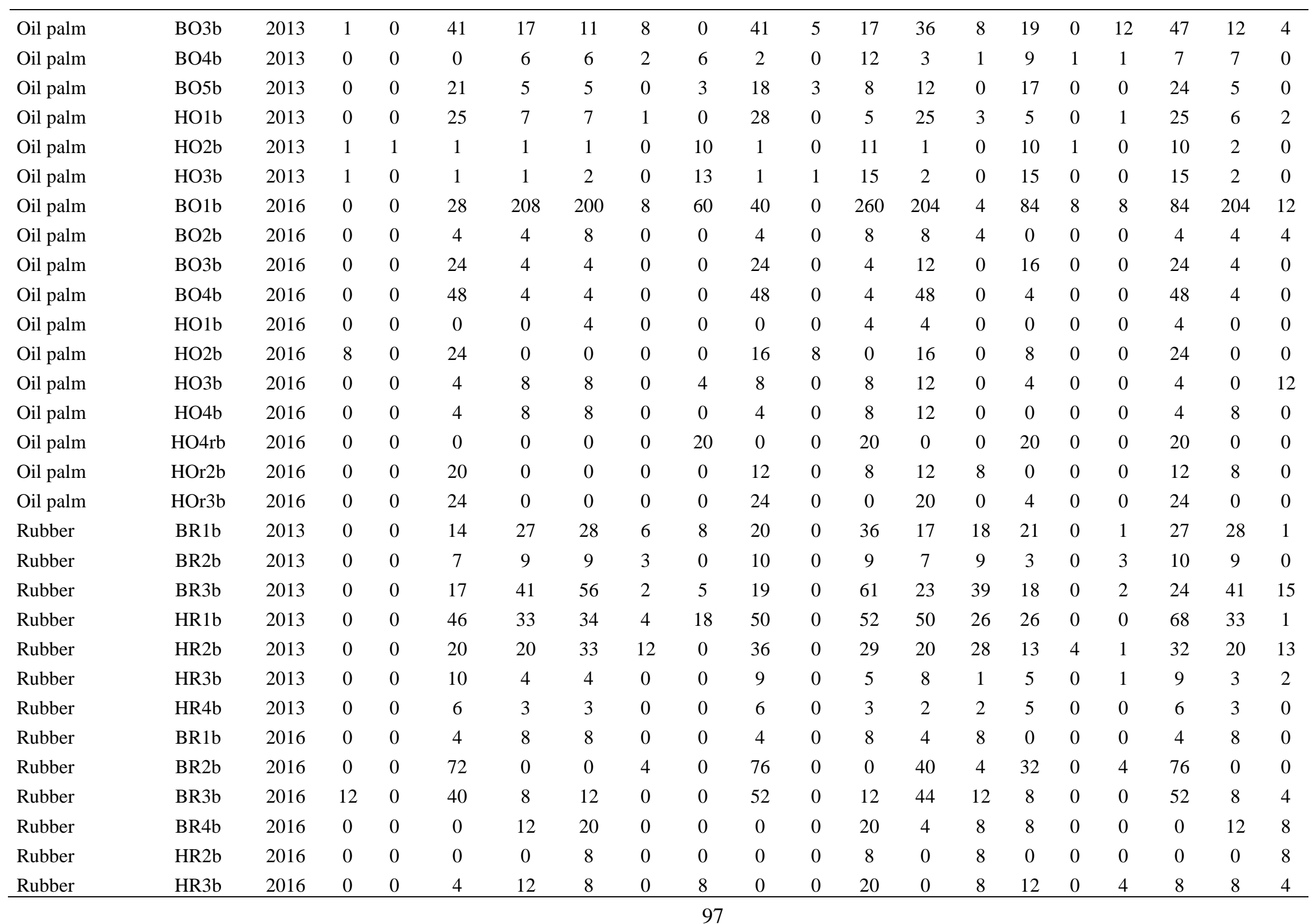


Chapter 3

\begin{tabular}{lcccccccccccccccccccccccccccc}
\hline Rubber & HR4b & 2016 & 0 & 0 & 4 & 4 & 8 & 0 & 4 & 4 & 0 & 12 & 4 & 8 & 4 & 0 & 0 & 8 & 4 & 4 \\
Rubber & HRr1b & 2016 & 0 & 0 & 48 & 84 & 48 & 0 & 0 & 12 & 0 & 84 & 16 & 44 & 36 & 0 & 36 & 12 & 48 & 36 \\
Rubber & HRr2b & 2016 & 8 & 0 & 12 & 12 & 12 & 0 & 8 & 20 & 0 & 20 & 20 & 12 & 8 & 0 & 0 & 28 & 12 & 0 \\
Rubber & HRr3b & 2016 & 0 & 0 & 0 & 12 & 20 & 0 & 0 & 0 & 0 & 20 & 0 & 20 & 0 & 0 & 0 & 0 & 12 & 8 \\
Rubber & HRr4b & 2016 & 0 & 0 & 20 & 16 & 16 & 0 & 0 & 20 & 0 & 16 & 8 & 16 & 12 & 0 & 0 & 20 & 16 & 0
\end{tabular}

Abbreviation of traits: Ea=Empodial appendage absent, Ms=Mouthpart sucking, PAOp= PAO present, Sp=Scales present, Ae=Abdoment IV elongated, $\mathrm{As}=$ Abdoment spherical, $\mathrm{Af}=\mathrm{Abdoment}$ fused, $\mathrm{Fs}=$ Furca straight, $\mathrm{Fa}=$ Furca absent, $\mathrm{Fl}=$ Furca long, $\mathrm{Pd}=\mathrm{Pigmenttaion}$ diffuse, $\mathrm{Pp}=\mathrm{Pigmentation}$ pattern, $\mathrm{Pa}=$ Pigmentation absent, $\mathrm{Pi}=$ Pigmenttaion intensive, $\mathrm{Am}=$ Antennae modified, $\mathrm{Ss}=$ Small size, Ms=Medium size, $\mathrm{Ls}=\mathrm{Large}$ size 
Chapter 3

Supplementary Table S3.5. Trait matrix of Collembola in soil layer based on density

\begin{tabular}{|c|c|c|c|c|c|c|c|c|c|c|c|c|c|c|c|c|c|c|c|c|}
\hline System & Plot & Year & $\mathrm{Ea}$ & Ms & PAOp & $\mathrm{Sp}$ & $\mathrm{Ae}$ & As & Af & Fs & $\mathrm{Fa}$ & $\mathrm{Fl}$ & $\mathrm{Pd}$ & $\mathrm{Pp}$ & $\mathrm{Pa}$ & $\mathrm{Pi}$ & $\mathrm{Am}$ & Ss & Ms & Ls \\
\hline Rainforest & BF1b & 2013 & 4 & 4 & 2 & 26 & 26 & 0 & 5 & 3 & 1 & 31 & 2 & 0 & 29 & 4 & 0 & 5 & 29 & 1 \\
\hline Rainforest & $\mathrm{BF} 2 \mathrm{~b}$ & 2013 & 0 & 0 & 34 & 12 & 11 & 2 & 17 & 20 & 15 & 29 & 9 & 5 & 49 & 1 & 2 & 52 & 8 & 4 \\
\hline Rainforest & $\mathrm{BF} 3 \mathrm{~b}$ & 2013 & 0 & 0 & 0 & 2 & 2 & 0 & 2 & 2 & 0 & 2 & 0 & 0 & 4 & 0 & 0 & 0 & 3 & 1 \\
\hline Rainforest & $\mathrm{BF} 4 \mathrm{~b}$ & 2013 & 1 & 0 & 94 & 66 & 66 & 3 & 385 & 5 & 93 & 451 & 5 & 2 & 542 & 0 & 0 & 483 & 64 & 2 \\
\hline Rainforest & $\mathrm{HF} 1 \mathrm{~b}$ & 2013 & 1 & 0 & 1 & 43 & 44 & 1 & 34 & 3 & 0 & 78 & 6 & 2 & 73 & 0 & 1 & 37 & 43 & 1 \\
\hline Rainforest & $\mathrm{HF} 2 \mathrm{~b}$ & 2013 & 3 & 1 & 1 & 52 & 51 & 2 & 41 & 5 & 0 & 93 & 14 & 3 & 80 & 1 & 3 & 45 & 53 & 0 \\
\hline Rainforest & HF3b & 2013 & 0 & 0 & 0 & 40 & 40 & 1 & 0 & 1 & 0 & 40 & 12 & 1 & 28 & 0 & 1 & 1 & 40 & 0 \\
\hline Rainforest & $\mathrm{HF} 4 \mathrm{~b}$ & 2013 & 8 & 0 & 0 & 85 & 85 & 1 & 5 & 9 & 0 & 90 & 37 & 1 & 61 & 0 & 1 & 14 & 85 & 0 \\
\hline Rainforest & $\mathrm{BF} 1 \mathrm{~b}$ & 2016 & 0 & 0 & 0 & 4 & 4 & 0 & 4 & 0 & 0 & 8 & 0 & 0 & 8 & 0 & 0 & 4 & 4 & 0 \\
\hline Rainforest & $\mathrm{BF} 2 \mathrm{~b}$ & 2016 & 12 & 0 & 20 & 24 & 8 & 0 & 12 & 16 & 0 & 36 & 24 & 0 & 28 & 0 & 16 & 28 & 8 & 16 \\
\hline Rainforest & $\mathrm{BF} 3 \mathrm{~b}$ & 2016 & 0 & 0 & 4 & 16 & 12 & 0 & 4 & 0 & 0 & 20 & 0 & 4 & 16 & 0 & 4 & 4 & 12 & 4 \\
\hline Rainforest & $\mathrm{BF} 4 \mathrm{~b}$ & 2016 & 12 & 0 & 12 & 8 & 8 & 0 & 4 & 0 & 12 & 12 & 0 & 0 & 24 & 0 & 0 & 16 & 8 & 0 \\
\hline Rainforest & $\mathrm{HF} 1 \mathrm{~b}$ & 2016 & 0 & 0 & 16 & 36 & 24 & 0 & 4 & 0 & 0 & 44 & 0 & 4 & 40 & 0 & 12 & 4 & 28 & 12 \\
\hline Rainforest & $\mathrm{HF} 2 \mathrm{~b}$ & 2016 & 0 & 0 & 12 & 16 & 4 & 0 & 0 & 0 & 0 & 16 & 0 & 4 & 12 & 0 & 12 & 0 & 4 & 12 \\
\hline Rainforest & $\mathrm{HF} 3 \mathrm{~b}$ & 2016 & 0 & 0 & 64 & 64 & 20 & 0 & 120 & 28 & 0 & 176 & 20 & 0 & 184 & 0 & 44 & 140 & 20 & 44 \\
\hline Rainforest & $\mathrm{HF} 4 \mathrm{~b}$ & 2016 & 44 & 16 & 40 & 72 & 64 & 12 & 88 & 44 & 16 & 156 & 28 & 44 & 128 & 16 & 20 & 144 & 64 & 8 \\
\hline Rainforest & HFr1b & 2016 & 0 & 0 & 0 & 0 & 4 & 0 & 0 & 0 & 0 & 4 & 0 & 4 & 0 & 0 & 0 & 0 & 0 & 4 \\
\hline Rainforest & HFr2b & 2016 & 0 & 0 & 4 & 32 & 28 & 0 & 4 & 0 & 0 & 36 & 0 & 8 & 28 & 0 & 4 & 4 & 28 & 4 \\
\hline Rainforest & $\mathrm{HFr} 3 \mathrm{~b}$ & 2016 & 0 & 0 & 4 & 8 & 8 & 0 & 0 & 4 & 0 & 8 & 4 & 8 & 0 & 0 & 0 & 4 & 8 & 0 \\
\hline Rainforest & $\mathrm{HFr} 4 \mathrm{~b}$ & 2016 & 0 & 0 & 0 & 4 & 4 & 0 & 8 & 0 & 0 & 12 & 0 & 4 & 8 & 0 & 0 & 8 & 4 & 0 \\
\hline Jungle rubber & $\mathrm{BJ} 2 \mathrm{~b}$ & 2013 & 0 & 0 & 14 & 27 & 26 & 0 & 2 & 14 & 0 & 28 & 14 & 1 & 27 & 0 & 2 & 14 & 25 & 3 \\
\hline Jungle rubber & $\mathrm{BJ} 3 \mathrm{~b}$ & 2013 & 2 & 2 & 13 & 27 & 30 & 0 & 12 & 16 & 0 & 41 & 14 & 11 & 30 & 2 & 0 & 25 & 29 & 3 \\
\hline Jungle rubber & $\mathrm{BJ} 4 \mathrm{~b}$ & 2013 & 0 & 0 & 36 & 0 & 0 & 1 & 2 & 39 & 0 & 0 & 32 & 0 & 6 & 1 & 0 & 37 & 0 & 2 \\
\hline Jungle rubber & BJ5b & 2013 & 2 & 1 & 5 & 55 & 55 & 2 & 1 & 7 & 0 & 57 & 29 & 5 & 29 & 1 & 2 & 8 & 54 & 2 \\
\hline Jungle rubber & $\mathrm{HJ} 1 \mathrm{~b}$ & 2013 & 0 & 0 & 17 & 5 & 5 & 0 & 6 & 17 & 0 & 11 & 14 & 3 & 11 & 0 & 0 & 23 & 5 & 0 \\
\hline Jungle rubber & $\mathrm{HJ} 3 \mathrm{~b}$ & 2013 & 0 & 0 & 6 & 4 & 4 & 0 & 2 & 6 & 0 & 6 & 0 & 1 & 11 & 0 & 0 & 8 & 4 & 0 \\
\hline Jungle rubber & $\mathrm{HJ} 4 \mathrm{~b}$ & 2013 & 6 & 0 & 8 & 9 & 9 & 0 & 1 & 3 & 6 & 9 & 0 & 2 & 16 & 0 & 1 & 8 & 8 & 2 \\
\hline
\end{tabular}


Chapter 3

\begin{tabular}{|c|c|c|c|c|c|c|c|c|c|c|c|c|c|c|c|c|c|c|c|c|}
\hline Oil palm & $\mathrm{BO} 2 \mathrm{~b}$ & 2013 & 4 & 0 & 17 & 52 & 50 & 7 & 7 & 21 & 4 & 58 & 15 & 3 & 59 & 6 & 3 & 26 & 57 & 0 \\
\hline Oil palm & $\mathrm{BO} 3 \mathrm{~b}$ & 2013 & 0 & 0 & 90 & 71 & 7 & 2 & 7 & 33 & 0 & 80 & 13 & 11 & 89 & 0 & 66 & 47 & 3 & 63 \\
\hline Oil palm & $\mathrm{BO} 4 \mathrm{~b}$ & 2013 & 4 & 4 & 25 & 25 & 31 & 10 & 14 & 17 & 16 & 48 & 11 & 17 & 45 & 8 & 7 & 47 & 27 & 7 \\
\hline Oil palm & $\mathrm{BO} 5 \mathrm{~b}$ & 2013 & 0 & 0 & 73 & 50 & 41 & 2 & 91 & 59 & 5 & 143 & 60 & 3 & 144 & 0 & 10 & 155 & 43 & 9 \\
\hline Oil palm & $\mathrm{HO} 1 \mathrm{~b}$ & 2013 & 0 & 0 & 24 & 30 & 16 & 2 & 21 & 25 & 1 & 53 & 26 & 18 & 33 & 2 & 16 & 49 & 28 & 2 \\
\hline Oil palm & $\mathrm{HO} 2 \mathrm{~b}$ & 2013 & 0 & 0 & 0 & 11 & 11 & 1 & 11 & 1 & 0 & 22 & 1 & 1 & 21 & 0 & 1 & 11 & 12 & 0 \\
\hline Oil palm & $\mathrm{HO} 3 \mathrm{~b}$ & 2013 & 0 & 0 & 24 & 60 & 44 & 0 & 0 & 8 & 0 & 60 & 44 & 0 & 24 & 0 & 16 & 8 & 44 & 16 \\
\hline Oil palm & $\mathrm{BO} 2 \mathrm{~b}$ & 2016 & 0 & 0 & 36 & 44 & 32 & 0 & 8 & 24 & 0 & 52 & 28 & 0 & 48 & 0 & 12 & 32 & 32 & 12 \\
\hline Oil palm & BO3b & 2016 & 0 & 0 & 12 & 24 & 20 & 4 & 0 & 12 & 0 & 24 & 8 & 16 & 12 & 0 & 8 & 8 & 24 & 4 \\
\hline Oil palm & $\mathrm{BO} 4 \mathrm{~b}$ & 2016 & 0 & 0 & 40 & 56 & 28 & 0 & 0 & 24 & 0 & 56 & 12 & 12 & 56 & 0 & 28 & 24 & 40 & 16 \\
\hline Oil palm & $\mathrm{BO} 1 \mathrm{~b}$ & 2016 & 0 & 0 & 16 & 0 & 0 & 0 & 0 & 0 & 0 & 16 & 0 & 16 & 0 & 0 & 0 & 0 & 16 & 0 \\
\hline Oil palm & $\mathrm{HO} 2 \mathrm{~b}$ & 2016 & 0 & 0 & 16 & 8 & 8 & 0 & 0 & 8 & 0 & 16 & 12 & 12 & 0 & 0 & 0 & 8 & 16 & 0 \\
\hline Oil palm & HO3b & 2016 & 8 & 0 & 20 & 4 & 8 & 0 & 24 & 32 & 8 & 12 & 8 & 4 & 40 & 0 & 0 & 24 & 4 & 24 \\
\hline Oil palm & HO4b & 2016 & 4 & 0 & 24 & 24 & 8 & 0 & 16 & 20 & 4 & 24 & 8 & 0 & 40 & 0 & 16 & 8 & 8 & 32 \\
\hline Oil palm & $\mathrm{HO} 4 \mathrm{rb}$ & 2016 & 12 & 0 & 24 & 100 & 100 & 0 & 60 & 12 & 12 & 160 & 72 & 4 & 108 & 0 & 0 & 84 & 100 & 0 \\
\hline Oil palm & HOr1b & 2016 & 0 & 0 & 40 & 24 & 0 & 0 & 4 & 16 & 0 & 28 & 16 & 0 & 28 & 0 & 24 & 20 & 0 & 24 \\
\hline Oil palm & HOr3b & 2016 & 0 & 0 & 48 & 40 & 16 & 0 & 0 & 20 & 0 & 44 & 20 & 4 & 40 & 0 & 24 & 20 & 20 & 24 \\
\hline Rubber & BR1b & 2013 & 1 & 1 & 12 & 37 & 31 & 2 & 22 & 9 & 0 & 59 & 11 & 22 & 34 & 1 & 6 & 30 & 32 & 6 \\
\hline Rubber & $\mathrm{BR} 2 \mathrm{~b}$ & 2013 & 0 & 0 & 21 & 18 & 18 & 2 & 3 & 23 & 0 & 21 & 16 & 1 & 27 & 0 & 0 & 26 & 17 & 1 \\
\hline Rubber & BR3b & 2013 & 0 & 0 & 7 & 42 & 43 & 1 & 0 & 8 & 0 & 43 & 33 & 1 & 17 & 0 & 0 & 8 & 42 & 1 \\
\hline Rubber & $\mathrm{BR} 4 \mathrm{~b}$ & 2013 & 0 & 0 & 5 & 8 & 8 & 0 & 0 & 5 & 0 & 8 & 4 & 0 & 9 & 0 & 0 & 5 & 8 & 0 \\
\hline Rubber & HR1b & 2013 & 0 & 0 & 24 & 63 & 46 & 2 & 20 & 10 & 0 & 82 & 7 & 12 & 73 & 0 & 19 & 29 & 42 & 21 \\
\hline Rubber & $\mathrm{HR} 2 \mathrm{~b}$ & 2013 & 0 & 0 & 7 & 28 & 25 & 6 & 0 & 8 & 0 & 30 & 2 & 20 & 16 & 0 & 5 & 8 & 23 & 7 \\
\hline Rubber & HR3b & 2013 & 0 & 0 & 16 & 23 & 20 & 0 & 0 & 10 & 0 & 26 & 8 & 1 & 27 & 0 & 6 & 12 & 17 & 7 \\
\hline Rubber & HR4b & 2013 & 0 & 0 & 3 & 22 & 20 & 0 & 0 & 1 & 0 & 22 & 0 & 0 & 23 & 0 & 2 & 1 & 20 & 2 \\
\hline Rubber & $\mathrm{BR} 1 \mathrm{~b}$ & 2016 & 0 & 0 & 0 & 12 & 12 & 0 & 8 & 0 & 0 & 20 & 4 & 8 & 8 & 0 & 0 & 8 & 12 & 0 \\
\hline Rubber & $\mathrm{BR} 2 \mathrm{~b}$ & 2016 & 0 & 0 & 48 & 52 & 24 & 0 & 0 & 20 & 0 & 52 & 16 & 0 & 56 & 0 & 28 & 20 & 24 & 28 \\
\hline Rubber & $\mathrm{BR} 3 \mathrm{~b}$ & 2016 & 0 & 0 & 16 & 4 & 4 & 0 & 0 & 16 & 0 & 4 & 16 & 0 & 4 & 0 & 0 & 16 & 4 & 0 \\
\hline Rubber & $\mathrm{BR} 4 \mathrm{~b}$ & 2016 & 0 & 0 & 20 & 56 & 36 & 0 & 0 & 0 & 0 & 56 & 32 & 0 & 24 & 0 & 20 & 0 & 36 & 20 \\
\hline Rubber & HR1b & 2016 & 0 & 0 & 4 & 52 & 52 & 0 & 0 & 4 & 0 & 52 & 4 & 12 & 40 & 0 & 0 & 4 & 52 & 0 \\
\hline
\end{tabular}


Chapter 3

\begin{tabular}{|c|c|c|c|c|c|c|c|c|c|c|c|c|c|c|c|c|c|c|c|c|}
\hline Rubber & $\mathrm{HR} 2 \mathrm{~b}$ & 2016 & 0 & 0 & 48 & 84 & 48 & 0 & 0 & 12 & 0 & 84 & 16 & 44 & 36 & 0 & 36 & 12 & 48 & 36 \\
\hline Rubber & HR3b & 2016 & 0 & 0 & 16 & 36 & 36 & 0 & 0 & 20 & 0 & 32 & 12 & 12 & 28 & 0 & 4 & 12 & 32 & 8 \\
\hline Rubber & $\mathrm{HR} 4 \mathrm{~b}$ & 2016 & 0 & 0 & 0 & 24 & 24 & 0 & 0 & 8 & 0 & 16 & 4 & 12 & 8 & 0 & 0 & 0 & 24 & 0 \\
\hline Rubber & HRr1b & 2016 & 0 & 0 & 24 & 20 & 16 & 0 & 0 & 4 & 0 & 36 & 4 & 24 & 12 & 0 & 12 & 4 & 16 & 20 \\
\hline Rubber & $\mathrm{HRr} 2 \mathrm{~b}$ & 2016 & 0 & 0 & 4 & 12 & 8 & 0 & 0 & 0 & 0 & 12 & 0 & 8 & 4 & 0 & 4 & 0 & 8 & 4 \\
\hline Rubber & $\mathrm{HRr} 3 \mathrm{~b}$ & 2016 & 0 & 0 & 16 & 40 & 48 & 0 & 0 & 16 & 0 & 48 & 12 & 24 & 28 & 0 & 4 & 12 & 36 & 16 \\
\hline Rubber & $\mathrm{HRr} 4 \mathrm{~b}$ & 2016 & 0 & 0 & 12 & 16 & 28 & 0 & 12 & 16 & 0 & 36 & 4 & 28 & 20 & 0 & 0 & 20 & 16 & 16 \\
\hline
\end{tabular}

Abbreviation of traits: Ea=Empodial appendage absent, Ms=Mouthpart sucking, PAOp= PAO present, $\mathrm{Sp}=\mathrm{Scales}$ present, Ae=Abdoment IV elongated,

As=Abdoment spherical, Af=Abdoment fused, Fs=Furca straight, $\mathrm{Fa}=$ Furca absent, Fl=Furca long, $\mathrm{Pd}=\mathrm{Pigmenttaion}$ diffuse, $\mathrm{Pp}=\mathrm{Pigmentation}$ pattern,

$\mathrm{Pa}=$ Pigmentation absent, $\mathrm{Pi}=$ Pigmenttaion intensive, $\mathrm{Am}=$ Antennae modified, $\mathrm{Ss}=$ Small size, Ms=Medium size, Ls=Large size 
Supplementary Figure S3.1 Rarefaction curves of Collembola species from different system of each year
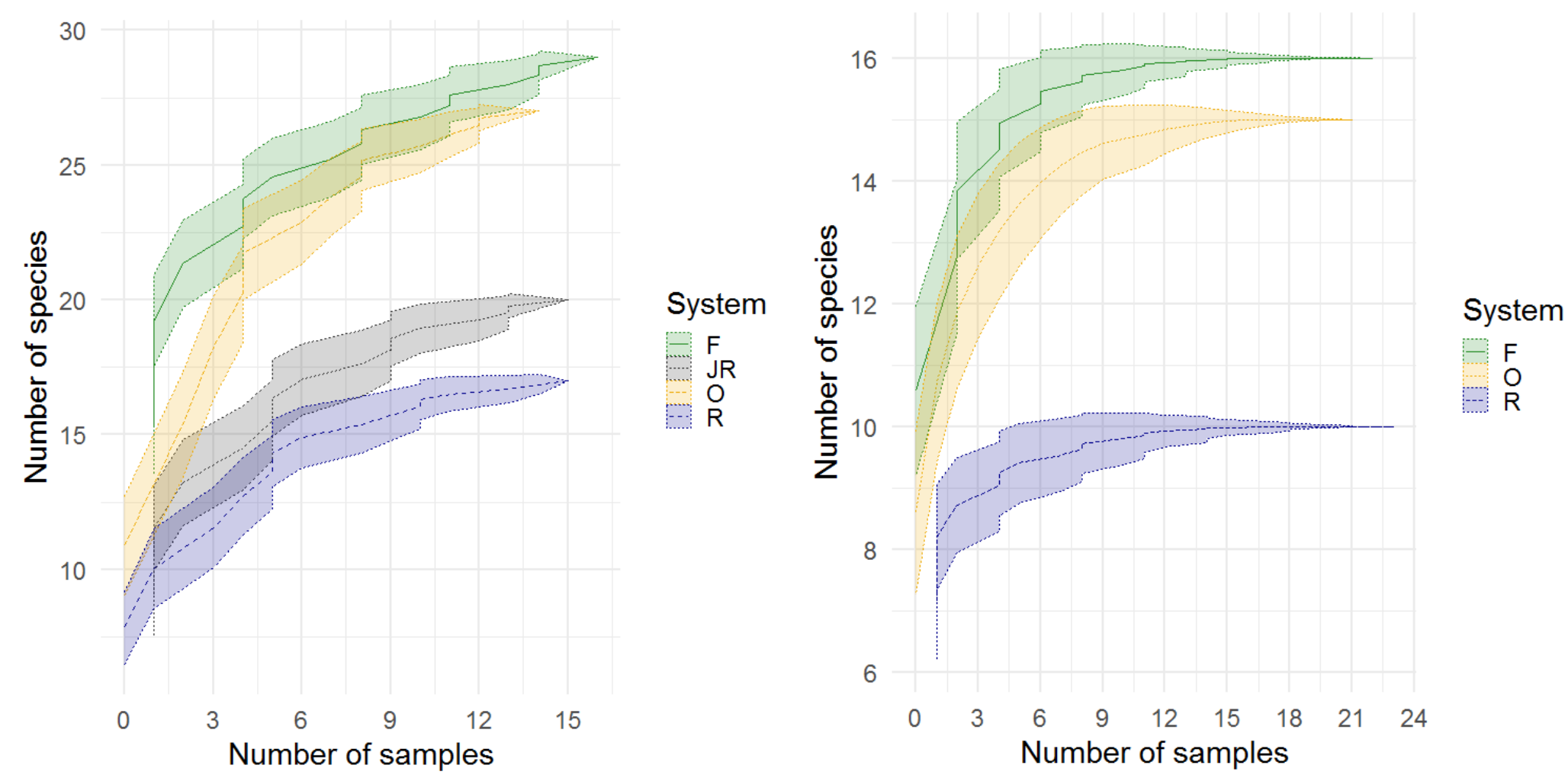

Supplementary Figure S3.1. Rarefaction curves of Collembola in different land-use systems in 2013 ( $F=$ Rainforest, JR=Jungle Rubber, $R=$ Rubber, $O=O$ Oil Palm) and 2016 (F=Rainforest, R=Rubber, O=Oil Palm) 


\section{CHAPTER 4}

TROPHIC NICHE DIFFERENTIATION AND UTILISATION OF FOOD RESOURCES IN COLLEMBOLA IS ALTERED BY RAINFOREST CONVERSION TO PLANTATION SYSTEMS

Winda Ika Susanti, Rahayu Widyastuti, Stefan Scheu, Anton Potapov
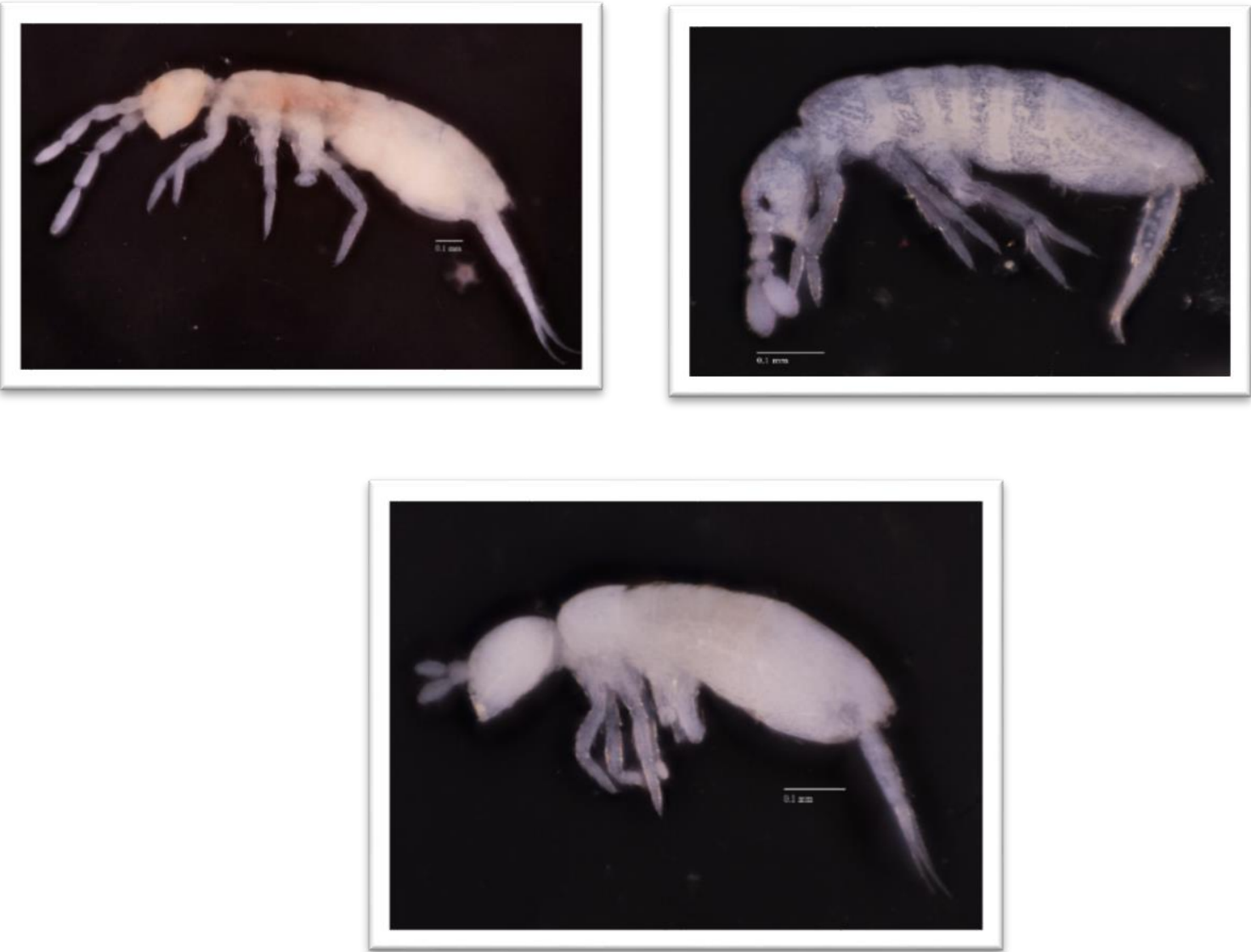

Photos by Winda Ika Susanti 


\section{TROPHIC NICHE DIFFERENTIATION AND UTILISATION OF FOOD RESOURCES IN COLLEMBOLA IS ALTERED BY RAINFOREST CONVERSION TO PLANTATION SYSTEMS}

\section{ABSTRACT}

Intensively managed monoculture plantations are increasingly replacing natural forests across the tropics resulting in changes in ecological niches of species and communities, and in ecosystem functioning. Collembola are among the most abundant arthropods inhabiting the belowground system sensitively responding to changes in vegetation and soil conditions. However, most studies on the response of Collembola to land-use change were conducted in temperate ecosystems and focused on shifts in community composition or morphological traits, while parameters more closely linked to ecosystem functioning, such as trophic niches, received little attention. Here, we used stable isotope analysis $\left({ }^{13} \mathrm{C}\right.$ and ${ }^{15} \mathrm{~N}$ ) to investigate changes in the trophic structure and use of food resources by Collembola in Jambi province (Sumatra, Indonesia), a region that experienced strong deforestation in the last decades. Isotopic values of Collembola from 32 sites representing four land-use systems were analyzed (rainforest, rubber agroforest, rubber (Hevea brasiliansis) and oil palm (Elaeis guineensis) monoculture plantations). Across Collembola species $\Delta^{13} \mathrm{C}$ values were highest in rainforest suggesting more pronounced processing of litter resources by microorganisms and consumption of these microorganisms by Collembola in this system. Lower $\Delta^{13} \mathrm{C}$ values, but high $\Delta^{13} \mathrm{C}$ variation in Collembola in oil palm plantations indicated that Collembola shifted towards herbivory and used more variable resources in this system. Small range in $\Delta^{15} \mathrm{~N}$ values in collembolan species in monoculture plantations in comparison to rainforest indicated that conversion of rainforest into plantations is associated with simplification in the trophic structure of Collembola communities. This was further confirmed by generally lower isotopic niche differentiation among species in plantations. Across the studied ecosystems, atmobiotic species (Symphypleona and Paronellidae) occupied the lowest, whereas euedaphic collembolan species occupied the highest trophic position, resembling patterns in temperate forests. Some species of Paronellidae in rainforest and jungle rubber had $\Delta^{15} \mathrm{~N}$ values below those of leaf litter suggesting algivory (Salina sp.1, Callyntrura sp.1 and Lepidonella sp.1), while a dominant species, Pseudosinella sp.1, had the highest $\Delta^{15} \mathrm{~N}$ values in most of the land-use systems suggesting that this species at least in part lives as predator or scavenger. Overall, the results suggest that rainforest conversion into 
plantation systems is associated with marked shifts in the structure of trophic niches in soil and litter Collembola with potential consequences for ecosystem functioning and food-web stability.

Key words: rainforest, oil palm, rubber, land-use change, stable isotope analysis, trophic niches, springtail

\section{Introduction}

Agricultural intensification in Indonesia is associated with deforestation which increased strongly in the last 30 years (Koh \& Ghazoul, 2010; Gatto et al., 2015). Large parts of rainforest in lowland Sumatra (Indonesia) have been converted into oil palm (Elaeis guineensis) (16\% of total area) and rubber plantations (Hevea brasiliansis) (12\%) (Gatto et al., 2015). These processes are driven by the high global demand for agricultural products, and positively affect income and employment of local smallholders (Grass et al., 2020; Qaim et al., 2020). At the same time, conversion of tropical rainforest into plantation systems is associated with major changes in ecological niches of animal species, loss of biodiversity, and thereby with changes in ecosystem functioning (Fitzherbert et al., 2008; Gilbert, 2012; Barnes et al., 2014; Clough et al., 2016). These changes affect both the above- and belowground system. Complex and diverse microbial and animal communities in soil regulate important ecosystem functions and support aboveground life (Bardgett and Van Der Putten, 2014), but knowledge on effects of landuse change on soil life in the tropics is very limited.

Studies from Sumatra showed that conversion of rainforest into oil palm and rubber plantations is associated with a decline in species diversity, population density, biomass and energy flux in litter macroinvertebrate communities by approximately $45 \%$ (Barnes et al., 2014). Uneven decline in energy flux across size classes and trophic levels was documented for meso- and macrofauna soil communities leading to strong alterations in soil food-web structure (Potapov et al., 2019). Different basal resources available in different land-use systems result in changes in trophic niches of decomposer and predatory soil invertebrates, and results in reduced abundance of primary decomposers and in soil animals shifting their feeding habits towards herbivory (Klarner et al., 2017; Krause et al., 2019; Susanti et al., 2019). Land-use change may also result in reduced 
trophic niche differentiation among species in belowground communities (Korotkevich et al., 2018), but this has not been investigated in tropical ecosystems.

Collembola are among the most abundant soil decomposer invertebrates, inhabiting various organic substrates and using a wide range of food resources (Rusek, 1998). Early studies on food resources of Collembola concluded that the majority of euedaphic and hemiedaphic species feed unselectively on a wide variety of food materials (Hopkin, 1997). However, stable isotope analysis showed pronounced trophic niche differentiation among collembolan species in temperate forests (Chahartagi et al., 2005). This differentiation in large has been explained by the taxonomic identity and life forms of Collembola (Potapov et al., 2016). Species, living aboveground and on the litter surface (atmobiotic and epedaphic life forms) are mainly phycophages, feeding on lichens, algae and pollen. Species, living in the litter (hemiedaphic life form) are detritivores feeding on saprotrophic microorganisms and litter. Species, living in soil (euedaphic life form) feed on soil organic matter, roots and fungi (Ponge, 2000; Potapov et al., 2016). Further, food resources and trophic levels also vary among high-rank taxa with e.g., Poduromorpha occupying higher trophic positions than Entomobryomorpha and Symphypleona, suggesting evolutionary selection for microbivory in the former (Potapov et al., 2016). Since lowland tropical forest ecosystems often have a less pronounced organic layer and different plant and animal community composition than temperate forest ecosystems (Petersen and Luxton, 1982), they provide different ecological niches for Collembola, potentially resulting in a different trophic structure of communities. To date, information on food resources and trophic niches of Collembola is based on studies from temperate ecosystems, whereas information from tropical ecosystems is virtually lacking entirely.

Over the last two decades, stable isotope analysis has become the most commonly used tool to assess trophic niches of soil animals (Potapov et al., 2019). Two isotopic ratios, ${ }^{13} \mathrm{C} /{ }^{12} \mathrm{C}$ (i.e., $\delta^{13} \mathrm{C}$ values) and ${ }^{15} \mathrm{~N} /{ }^{14} \mathrm{~N}$ (i.e., $\delta^{15} \mathrm{~N}$ values), typically are used in foodweb studies. Trophic positions and length of trophic chains can be assessed using $\delta^{15} \mathrm{~N}$ values, whereas the range of $\delta^{13} \mathrm{C}$ values reflects variability in the use of basal resources (Potapov et al., 2019). Stable isotope composition of consumers follows that in food resources, thus allowing to reveal potential diet switching with land-use change (Klarner et al., 2017; Krause et al., 2019; Susanti et al., 2019).

Here, we use stable isotope analysis to investigate trophic positions and food resources of soil and litter Collembola in four different land-use systems in Sumatra, 
Indonesia: rainforest, rubber agroforest ('jungle rubber'), and monoculture rubber and oil palm plantations. The study aimed at investigating how trophic positions and food resources of Collembola change after rainforest conversion into agricultural plantations, such as rubber and oil palm, and, for the first time, at exploring patterns in trophic niche differentiation among tropical collembolan species. In more detail we investigated the following hypotheses:

1) Analogous to other soil invertebrates, Collembola shift their trophic niches towards herbivory in plantation systems in comparison to rainforest.

2) Due to reduced food resources (poor litter layer), the trophic niche width of Collembola is narrower in plantations in comparison to rainforest.

3) Trophic niche differentiation among collembolan species is more pronounced in rainforest than in plantation systems.

4) Trophic niche differentiation among families and life forms of Collembola in tropical ecosystems follows similar patterns as in ecosystems of the temperate zone.

\section{Material and Methods}

\section{Site description}

Four land-use systems were investigated: lowland rainforest, jungle rubber, rubber and oil palm plantations, located in Jambi province, southwest Sumatra, Indonesia. The study sites were located at a similar altitude varying between 50 and $100 \mathrm{~m}$ a.s.1. in two landscapes, the Harapan and Bukit Dua Belas landscape; each land-use system was replicated four times per landscape, resulting in a total of 32 sites (for more details see Drescher et al., 2016). Lowland rainforest was used as reference, but represents secondary rainforest, which has been logged once by taking out large trees some 30 years ago. Jungle rubber represents a rubber agroforest system originating from rainforest enriched with rubber trees; the age of rubber trees varied between 15-40 years (Kotowska et al., 2015). Rubber and oil palm plantations were intensively managed monocultures of an average age of 7 to 16 and 8 to 15 years, respectively (Drescher et al., 2016), and were established after logging, clearing, and burning of either rainforest or jungle rubber. Soils at the Harapan landscape are loam Acrisols of low fertility, whereas in Bukit Dua Belas the major soil type is clay Acrisol (Allen et al., 2015; Kotowska et al., 2015). Management practices in these smallholder monoculture plantations are described in more detail in 
Allen et al. (2015). Oil palm plantations typically were fertilized once in the rainy season and once in the dry season. Typically, 300-500 kg NPK complete fertilizer, $300 \mathrm{~kg} \mathrm{KCl}$ and $138 \mathrm{~kg}$ urea $\left(\mathrm{CO}\left(\mathrm{NH}_{2}\right)_{2}\right)$ were added per hectare and year. Rubber and oil palm plantations were weeded manually or chemically throughout the year. The most commonly used herbicides were paraquat and glyphosate; these were applied at an average rate of 2 to $5 \mathrm{~L} \mathrm{ha}^{-1} \mathrm{y}^{-1}$ (Allen et al., 2015; Kotowska et al., 2015; Clough et al., 2016).

\section{Sampling procedure}

Samples were taken in October 2013 in three $5 \times 5$ m subplots within 50 x $50 \mathrm{~m}$ plots established at each study site (Drescher et al., 2016). In each subplot soil samples of $16 \times 16 \mathrm{~cm}$ were taken including the litter layer and the underlying top soil to a depth of $5 \mathrm{~cm}$. Animals from both layers were pooled for stable isotope analysis to obtain sufficient amount of animal tissue for the analyses. Animals were extracted by heat (Kempson et al., 1963) until the substrate was completely dry (6-8 days) using glycerol : water mixture at a ratio of $1: 1$ as collection solution. Field collection was conducted under the research permit No. 389/SIP/FRP/SM/X/2013 issued by the State Ministry of Research and Technology of Indonesia (RISTEK) with collection permit No. S.07/KKH2/2013 issued by the Ministry of Forestry (PHKA) and support from the following persons and organizations who granting us to access and use their properties: village leaders, local plot owners, PT Humusindo, PT Perkebunan Nusantara VI, Harapan Rainforest, and Bukit Duabelas National Park.

\section{Species identification}

Collembola were sorted in Petri dishes using a dissecting microscope. For specieslevel identification, selected individuals were subsequently cleared in Nesbitt solution and mounted on slides with Hoyer solution. Collembola were identified under a compound light microscope at 400× magnification. The checklist and keys for Indonesian Collembola by Suhardjono et al. (2012) were used along with publications on Southeast Asian Collembola. Due to a relatively poorly described fauna, in many cases we had to assign individuals to morphospecies without Linnaean names (in total $72 \%$ of all identified species); for simplicity, we refer to both as 'species'. When possible, juvenile specimens were ascribed to species of adults or subadults present in the same sample or 
in samples from the same plot. After identification, all data on collembolan species and their identification characters were uploaded to Ecotaxonomy database (http://ecotaxonomy.org). In total 56 species from 13 families and 27 genera were found.

\section{Bulk stable isotope analyses}

Stable isotope ratios were- measured from dominant species representing at least $70 \%$ of the individuals on each plot (Supplementary Table S4.1). A number of rare species were observed only on few sites and such data would be not suitable for a proper analysis of the species and land-use effects and also for analytical facilities in the laboratory. Dominant species were chosen for each plot separately to represent the local 'functional community'. This selection procedure resulted in a total of 30 out of 56 species being included in the analysis across all land-use systems. For stable isotope measurements appropriate amounts of animal tissue (ranging from 0.003 to $1.268 \mathrm{mg}$ ) were transferred into tin capsules and dried at $60^{\circ} \mathrm{C}$ for $24 \mathrm{~h}$, weighed and stored in a desiccator until analysis. Stable isotope ratios, and total $\mathrm{C}$ and $\mathrm{N}$ concentration were determined using a coupled system consisting of an elemental analyzer (Eurovector, Milano, Italy) equipped with a Blisotec autosampler (Blisotec, Jülich, Germany) and a Thermo Delta Vplus isotope ratio mass spectrometer connected via a Conflo IV interface (both from Thermo Fisher Scientific, Bremen, Germany) located at the Centre for Stable Isotope Research and Analysis, Göttingen, Germany (Langel \& Dyckmans, 2014). Isotope signatures were expressed using the $\delta$ notation as $\delta \mathrm{X}(\% \mathrm{\%})=\left(\mathrm{R}_{\text {sample }}-\right.$ $\left.\mathrm{R}_{\text {standard }}\right) / \mathrm{R}_{\text {standard, }}$ with $\mathrm{X}$ representing the target isotope and $\mathrm{R}$ the ratio of heavy to light isotope $\left({ }^{13} \mathrm{C} /{ }^{12} \mathrm{C}\right.$ or $\left.{ }^{15} \mathrm{~N} /{ }^{14} \mathrm{~N}\right)$. For $\delta^{15} \mathrm{~N}$ and $\delta^{13} \mathrm{C}$ analyses, $\mathrm{N}$ in atmospheric air and Vienna Pee Dee Belemnite served as standards, respectively. We use IAEA CH6 (-10.43\%, Sucrose) and IAEA 600 (-27.7\%o, Caffein) as C standards, and IAEA N1 (0.4\%o) and IAEA N2 (20.3\%) for N (both are Ammonium sulfates) for internal calibration.

\section{Statistical analysis}

To compensate for inter-site variation in the isotopic baseline, prior to the analysis all data were normalized to the local leaf litter using the following equations (Potapov et al., 2019):

$\Delta^{13} \mathrm{C}=\delta^{13} \mathrm{C}_{\text {Collembola }}-\delta^{13} \mathrm{C}_{\text {litter }}$ 
$\Delta^{15} \mathrm{~N}=\delta^{15} \mathrm{~N}_{\text {Collembola }}-\delta^{15} \mathrm{~N}_{\text {litter }}$

Stable isotope values of litter were taken from Klarner et al. (2017) who investigated the same sampling sites.

Statistical analyses were performed using R v 3.5.2 (R Core Team, 2018) with R studio interface (R Studio, Inc.). First, we analyzed the effect of land-use system on the isotopic composition of Collembola at the community level. Effects of land-use system on the $\Delta^{13} \mathrm{C}$ and $\Delta^{15} \mathrm{~N}$ values of all measured Collembola individuals were tested using Linear Mixed Effect Models (LMM) with species identity as random effect. Species identity was coded as random effect for the following reasons: (1) In this first analysis we were not interested in variations in $\Delta{ }^{13} \mathrm{C}$ and $\Delta{ }^{15} \mathrm{~N}$ values among species but still wanted to account for it, (2) species presence was uneven across plots and land-use systems preventing the analysis of land-use effects, and (3) including species as fixed factor would have compromised the analysis of land-use effects on $\Delta^{13} \mathrm{C}$ and $\Delta^{15} \mathrm{~N}$ values of Collembola by reducing error degrees of freedom. The analysis was conducted using the lmer function in the lme4 package (Bates et al., 2015). Significance of fixed effects (factors) was tested using the Anova function in the car package. Significant differences in stable isotope values between land-use systems were tested using the tukey test in the emmeans and multicomp packages. We also analyzed the effect of land use only for one dominant species, present in sufficient replicates in each land-use system (Pseudosinella sp.1) using analysis of variance implemented in the aov function. Additionally, the ranges in $\Delta^{13} \mathrm{C}$ and $\Delta^{15} \mathrm{~N}$ values in each land-use system were calculated as difference between minimum and maximum values and visualized using the Kernel density estimation in the ggplot 2 package using the geom_violin function.

Second, we assessed trophic niche differentiation among species by assessing the effect of species identity on stable isotope composition of Collembola with LMM. We used species identity as factor and either $\Delta^{13} \mathrm{C}$ or $\Delta^{15} \mathrm{~N}$ values as response variables; sampling plot was included as random effect to account for site-specific differences in trophic niches. The analysis was done separately for each land-use system (eight analyses in total). Only species allowing more than three measurements per land-use system were included.

Third, we analyzed the effects of family identity and life form on stable isotope composition of Collembola using LMM. Here, we used both plot and land-use system as random effects. Significant differences between families and life forms were tested using 
Tukey contrasts as implemented in the glth function in the multicomp package. To display the isotopic niche space of collembolan species, family, and lifeform, ellipses denoting $60 \%$ intervals were plotted using the standard.ellipse function in the siar package, and visualized using the ggplot and ggrepel packages. We also analyzed interactions between land-use system and life form and between land-use system and family with plot as random effect. In the analyses, we excluded Neanuridae and Onychiuridae as they were only represented by a single species not present in each of the land-use systems. In addition, we also analyzed $\mathrm{R}$ square values between species, family, and lifeform to identify the most important factor. Here, we used plot as random effect The analysis was done using the r.squaredGLMM function in the lme4 and MuMin packages.

\section{Results}

\section{Community-level changes with land use}

In rainforest $\Delta^{13} \mathrm{C}$ values of Collembola ranged between 1.5 and $6.0 \%$ (total range $4.5 \%$ ), and in oil palm plantations between -1.5 and $8.5 \%$ (10.0\%o), indicating a wider range of $\Delta^{13} \mathrm{C}$ values in food resources in the latter. In jungle rubber and rubber plantations, the range of $\Delta^{13} \mathrm{C}$ values was similar to rainforest (between 1.0 and $6.0 \%$ ). Mean $\Delta^{13} \mathrm{C}$ values in rainforest were significantly higher than in plantation systems $\left(\mathrm{F}_{3,146}\right.$ $=9.90, \mathrm{p}=0.001 ;$ Fig. 4.1A, Supplementary Material Tables S4.2, S4.3). All $\delta^{13} \mathrm{C}$ values of Collembola species exceeded those of leaf litter except for one individual of Isotomiella cf. minor in oil palm plantations (Fig. 4.2; for details see Supplementary Material Tables S4.6-S4.9).

In contrast to $\Delta^{13} \mathrm{C}$, the range of $\Delta^{15} \mathrm{~N}$ values was largest in rainforest $(-5.0$ to $19.0 \%$ ), lowest in oil palm plantations (-1.0 to 8.0\%o), and intermediate in rubber plantations (-5.0 to $15.0 \%$ o) and in jungle rubber (-7.5 to $16.0 \%$ ). Mean $\Delta^{15} \mathrm{~N}$ values of Collembola did not vary significantly among land-use systems $\left(F_{3,153}=1.46, p=0.228\right.$; Fig. 4.1B, Supplementary Material Tables S4.4, S4.5). Stable isotope values of the most abundant collembolan species present in all land-use systems, Pseudosinella sp.1, did not vary significantly among land-use systems both in $\Delta^{13} \mathrm{C}\left(\mathrm{F}_{3,21}=1.44, \mathrm{p}=0.260\right)$ and $\Delta^{15} \mathrm{~N}$ $\left(\mathrm{F}_{3,21}=0.88, \mathrm{p}=0.467\right)$. 


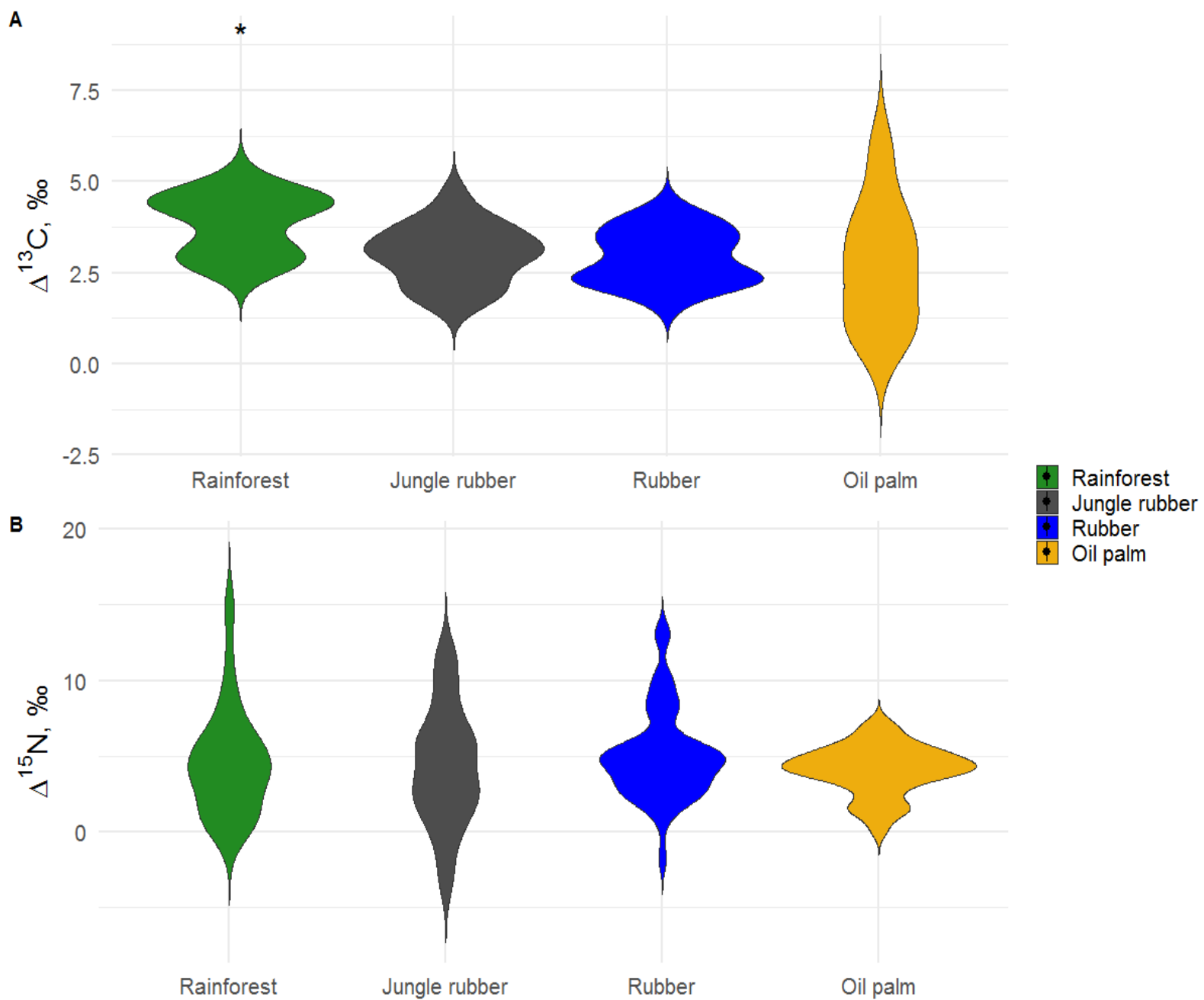

Figure 4.1. Variations in $113 \mathrm{C}$ and $115 \mathrm{~N}$ values of Collembola among the studied land-use systems: (A) rainforest, (B) jungle rubber, (C) rubber and (D) oil palm plantations. Violin plots show frequency distribution of values (mirrored Kernel density estimation), all individual measurements are displayed together independent of taxonomic identity. *Average $113 \mathrm{C}$ values in rainforest were significantly higher than in the other three land-use systems $(\mathrm{P}<0: 05)$.

\section{Trophic niche differentiation among species}

$\Delta^{13} \mathrm{C}$ values across abundant species (represented by at least three measurements, see Methods) varied significantly in rainforest $\left(\mathrm{F}_{4,12}=6.34, \mathrm{p}=0.005\right)$ and jungle rubber $\left(\mathrm{F}_{5,17}=3.67, \mathrm{p}=0.020\right)$, but not in rubber $\left(\mathrm{F}_{5,19}=1.39, \mathrm{p}=0.270\right)$ and oil palm plantations $\left(\mathrm{F}_{2,9}=1.10, \mathrm{p}=0.370\right)$. In rainforest, $\Delta^{13} \mathrm{C}$ values of Pseudosinella sp.1 were highest and differed significantly from those of Lepidocyrtus sp.1 and Pararrhopalites sp.1, whereas in jungle rubber they were highest in Homidia cingula and differed significantly from Callyntrura sp.1. 
$\Delta{ }^{15} \mathrm{~N}$ values across abundant species varied significantly in rainforest $\left(\mathrm{F}_{4,14}=5.00\right.$, $\mathrm{p}=0.010)$, jungle rubber $\left(\mathrm{F}_{5,18}=4.70, \mathrm{p}=0.006\right)$ and oil palm plantations $\left(\mathrm{F}_{2,9}=23.59\right.$, $\mathrm{p}=0.001)$, but not in rubber plantations $\left(\mathrm{F}_{5,19}=2.43, \mathrm{p}=0.072\right)$ (Fig. 3; for details see Supplementary Material Tables S4.10-S4.13). In rainforest Pseudosinella sp.1 occupied the highest trophic position, followed by Isotomiella cf. minor, whereas Allacma sp.1 occupied the lowest trophic position. Similar to rainforest, in jungle rubber Pseudosinella sp.1 also occupied the highest trophic position, but $\Delta^{15} \mathrm{~N}$ values were lowest in Callyntrura sp.1. Overall, Pseudosinella sp.1, the most dominant species, occupied the highest trophic position across all species. Across all samples analyzed (20 species in rainforest, 17 species jungle rubber, 15 species in rubber plantations, and 13 species in oil palm plantations) $3 \%$ of the measurements had $\Delta^{15} \mathrm{~N}$ values below that of litter (see Supplementary Material, Fig. 4.1). 


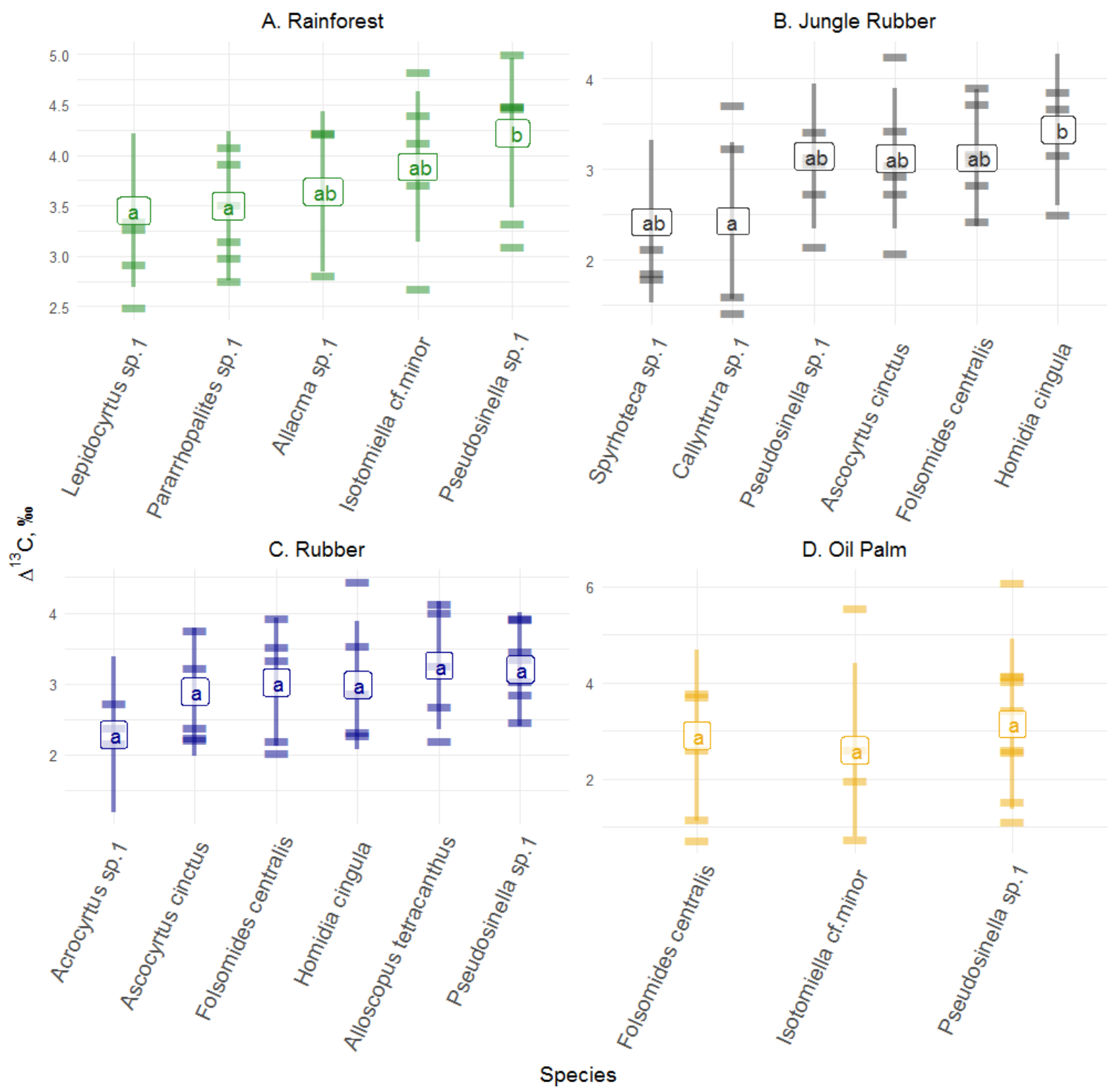

Figure 4.2. Differences of $113 \mathrm{C}$ values of Collembola species in different land-use systems: (A) rainforest, (B) jungle rubber, (C) rubber and (D) oil palm plantations; model-estimated means (lsmeans) with standard deviation. Horizontal stripes represent individual measurements. Only species with more than three replicates per land-use system were analyzed (see Methods). Isotope values of each species in each land-use system with the same letter are not significantly different according to Tukey's honestly significant difference test $(\mathrm{p}>0.05)$. 


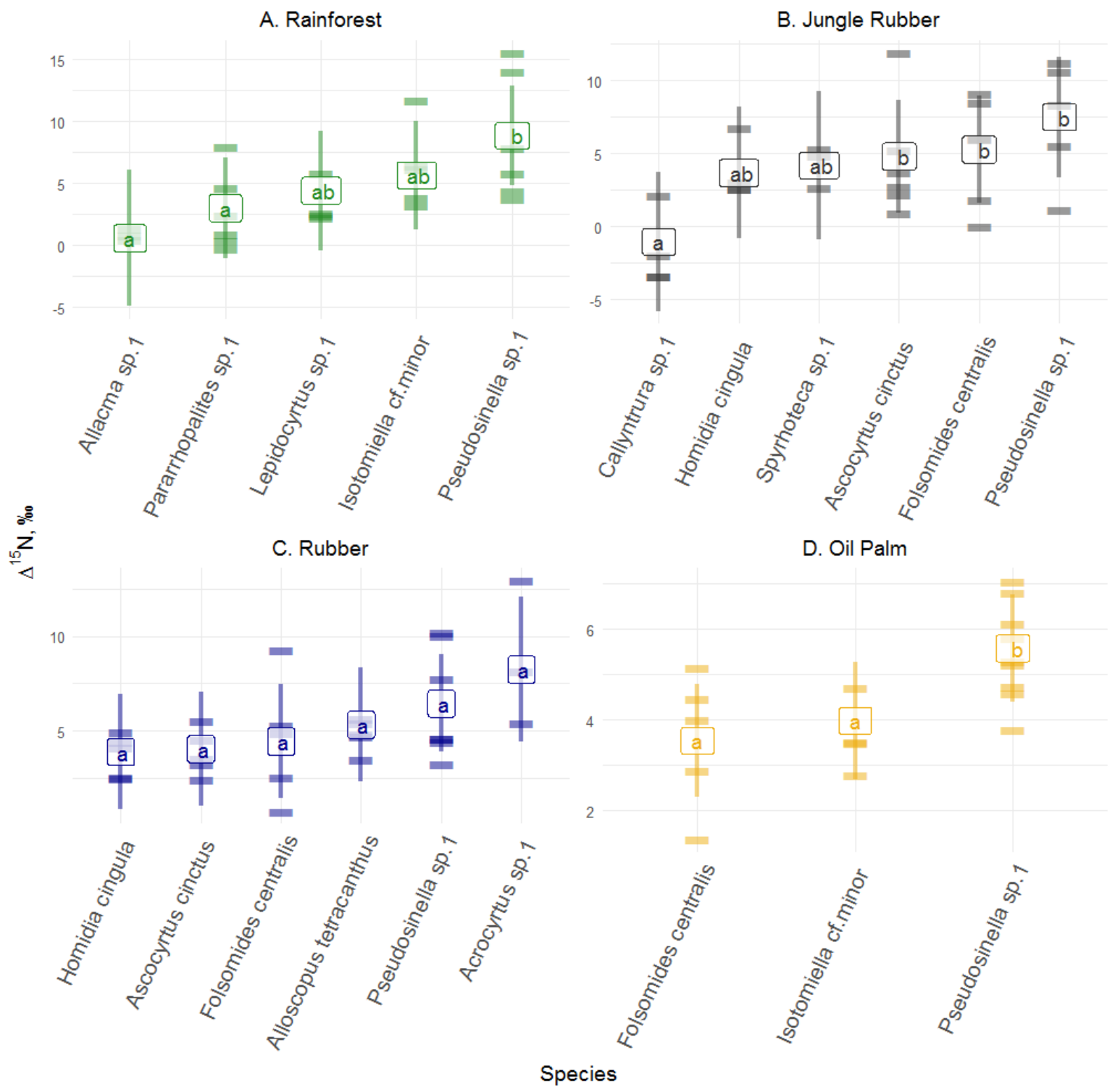

Figure 4.3. Differences of $115 \mathrm{~N}$ values of Collembola in different landuse systems: (A) rainforest, (B) jungle rubber, (C) rubber and (D) oil palm plantation; model-estimated means (lsmeans) with standard deviation. Horizontal stripes represent individual measurements. Only species with more than three replicates per land-use system were analyzed (see Methods). Isotope values of each species in each landuse system with the same letter are not significantly different according to Tukey's honestly significant difference test $(p>0.05)$. 


\section{Chapter 4}

\section{Differences among Collembola life forms and families}

The $\Delta{ }^{15} \mathrm{~N}$ values of Collembola varied among life forms $\left(\mathrm{F}_{24,126}=1.82, \mathrm{p}=0.018\right)$, but this was not the case for $\Delta^{13} \mathrm{C}$ values $\left(\mathrm{F}_{24,126}=1.19, \mathrm{p}=0.260\right)$, with the interaction between lifeform and land-use system neither being significant for $\Delta^{15} \mathrm{~N}\left(\mathrm{~F}_{9,132}=0.87, \mathrm{p}=0.556\right)$ nor for $\Delta^{13} \mathrm{C} \quad\left(\mathrm{F}_{9,135}=0.79, \mathrm{p}=0.619\right)$. Differences between life forms were more pronounced in rainforest and jungle rubber and less in rubber and oil palm plantations (Fig. 4.4). Euedaphic species were generally most enriched in ${ }^{15} \mathrm{~N}$, whereas atmobiotic species on average occupied the lowest trophic position (except in rubber plantations), often having $\Delta^{15} \mathrm{~N}$ values below 5.0\%, with epedaphic and hemiedaphic species being intermediate.

Similar to results of the life form analysis, the $\Delta^{15} \mathrm{~N}\left(\mathrm{~F}_{24,129}=2.19, \mathrm{p}=0.002\right)$ but not $\Delta^{13} \mathrm{C}$ values $\left(\mathrm{F}_{24,128}=1.01, \mathrm{p}=0.470\right)$ of Collembola varied significantly among families (Fig. 4.5), with the interaction between family and land-use system neither being significant for $\Delta^{15} \mathrm{~N}$ $\left(\mathrm{F}_{8,122}=0.34, \mathrm{p}=0.945\right)$ nor for $\Delta^{13} \mathrm{C}\left(\mathrm{F}_{8,121}=1.84, \mathrm{p}=0.075\right)$. Isotomidae and Entomobryidae occupied the highest trophic positions across land-use systems, Symphypleona occupied the lowest trophic position in rainforest and oil palm plantations, whereas in jungle rubber and rubber plantations the lowest trophic position was occupied by Paronellidae. Family and lifeform identity explained approximately two times less variation in $\Delta^{15} \mathrm{~N}$ values than species identity with $\mathrm{R}^{2}=0.29$ in models based on species, and $\mathrm{R}^{2}=0.13$ in both models based on families and life forms. 


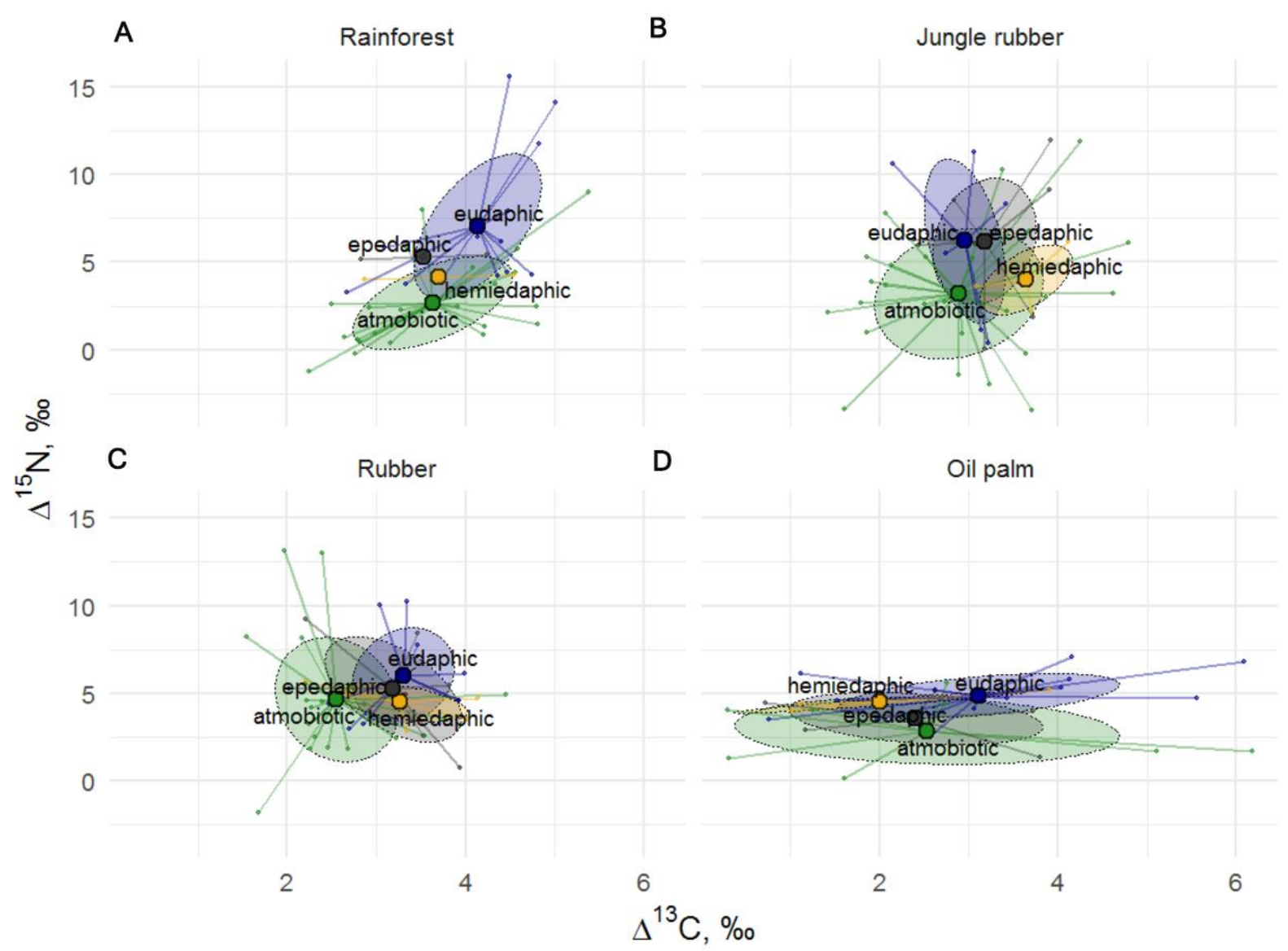

Figure 4.4. Stable isotope niches of four collembolan life forms in (A) rainforest, (B) jungle rubber, (C) rubber, and (D) oil palm plantations. Ellipses denote $60 \%$ confidence intervals, different life forms are shown in color (eudaphic D blue, epedaphic D grey, hemiedaphic D yellow, atmobiotic D green). Large colored dots represent means of land-use systems, small points represent individual measurements. 


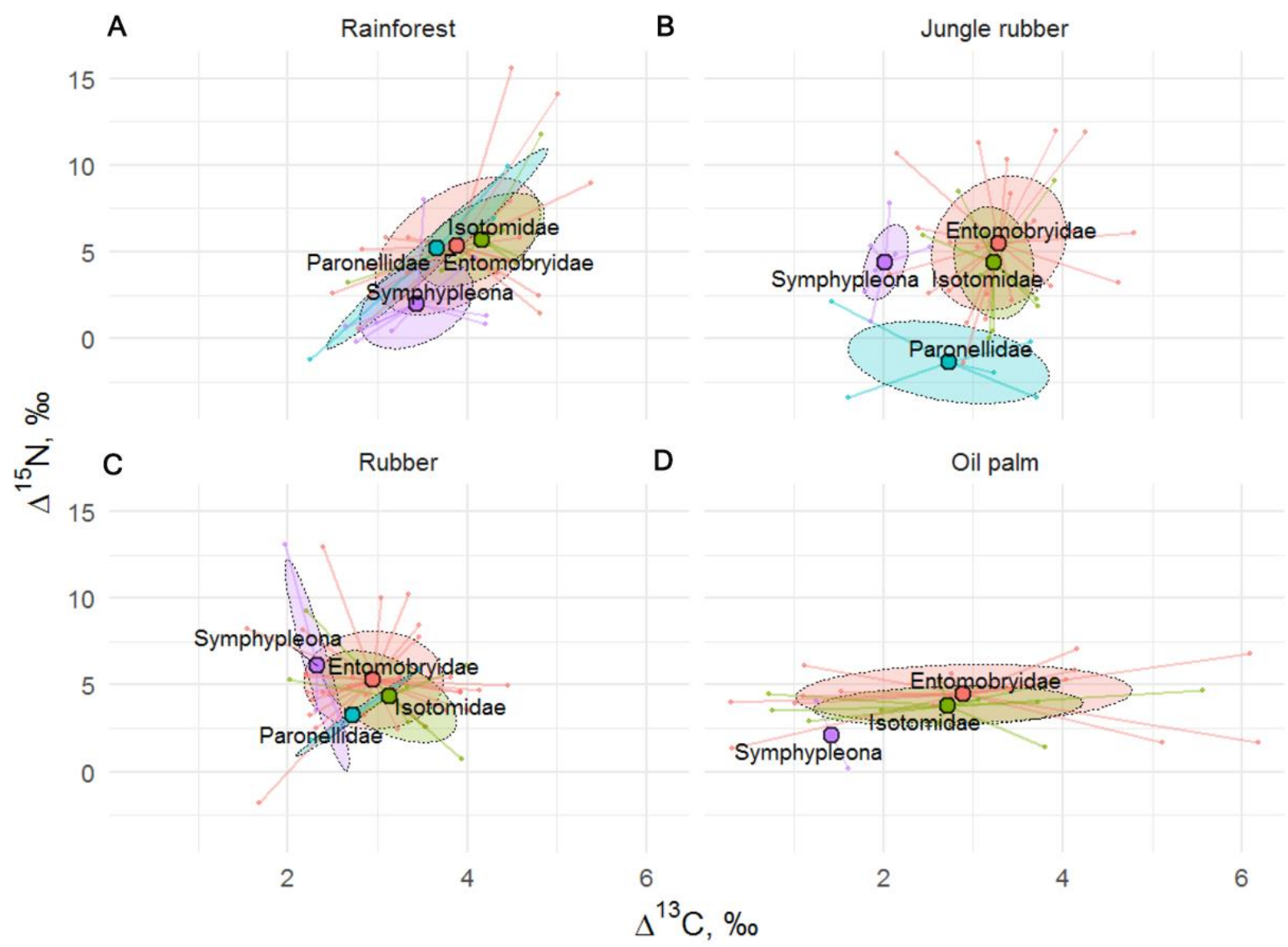

Figure 4.5. Stable isotope niche of collembolan families in (A) rainforest, (B) jungle rubber, (C) rubber, and (D) oil palm plantations. Ellipses denote 60\% intervals, different families are shown in color (Paronellidae D blue, Isotomidae D green, Symphypleona D purple, Entomobryidae D red; Symphypleona comprises the families Sminthuridae, Sminthiridididae, Dycirtomidae and Katiannidae). Large colored dots represent means of land-use systems, small points represent individual measurements.

\section{Discussion}

\section{Variations in trophic niches with land-use system}

Results of our study indicate that rainforest conversion into agricultural plantations is associated with changes in basal resources $\left(\Delta^{13} \mathrm{C}\right.$ values $)$ of Collembola, but does not significantly affect their average trophic positions ( $\Delta^{15} \mathrm{~N}$ values). These findings are in line with the results of the study of Krause et al. (2019) investigating oribatid mites at the same study sites and showing that the shift in trophic niches to be mainly due to changes in the use of basal resources rather than trophic levels. Similar to previous studies on centipedes, oribatid mites and other soil animal taxa (Klarner et al., 2017; Susanti et al., 2019; Krause et al., 2019), we also found the conversion of rainforest into plantations to be associated in Collembola with a 
shift from detritivory towards herbivory (i.e., lower ${ }^{13} \mathrm{C}$ enrichment). In rainforest and jungle rubber, $\delta^{13} \mathrm{C}$ values of Collembola were 2.0-5.0\% higher compared to leaf litter, which resembles the shift observed in temperate forest ecosystems (Pollierer et al., 2009). This "detrital shift" in $\delta^{13} \mathrm{C}$ (Potapov et al., 2019) presumably is due to acquiring $\mathrm{C}$ from saprotrophic fungi and bacteria (Potapov et al., 2013) that preferentially use ${ }^{13} \mathrm{C}$-rich plant compounds (Pollierer et al., 2009). High ${ }^{13} \mathrm{C}$ enrichment in most of the studied collembolan species in more natural ecosystems (rainforest and jungle rubber) suggests that they predominantly rely on microorganisms decomposing organic matter rather than on fresh plant material as food source (Potapov et al., 2019). Overall, similar enrichment in ${ }^{13} \mathrm{C}$ and ${ }^{15} \mathrm{~N}$ in Collembola in temperate and tropical ecosystems suggest that Collembola rely little on fresh plant material or mycorrhizal fungi in both, and this may well apply to soil food webs in general.

As indicated by $\Delta^{13} \mathrm{C}$ values, collembolan species in oil palm plantations used food resources of a wide range of ${ }^{13} \mathrm{C}$ values, which was not the case in the other land-use systems studied. Variations in ${ }^{13} \mathrm{C}$ values of food resources in oil palm plantations may be attributed to the variety of management practices of the smallholder systems studied (Clough et al., 2016). Potentially, variations in organic inputs due to differences in weeding, herbicide, and fertilization practices, resulted in an overestimation of the trophic niche width in oil palm plantations.

The narrow range of $\Delta^{15} \mathrm{~N}$ values in oil palm plantations suggests similar trophic positions of collembolan species in this land-use system. Notably, the narrow range was due to both the lack of high trophic level (predators, scavengers) and low trophic level species (primary decomposers, specialized lichen feeders). This may reflect the dominance of generalist species in the disturbed habitat of oil palm plantations (Korotkevich et al., 2018). Nematode communities also indicated oil palm plantations to be the most disturbed of the four land-use systems studied (Krashevska et al., 2019). Further, Klarner et al. (2017) found $\Delta^{15} \mathrm{~N}$ values of centipede species to be lower in oil palm compared to jungle rubber and rubber plantations suggesting that trophic chains in oil palm plantations are shorter than in the other land-use systems studied. Overall, these findings suggest that the structure of soil food-webs in monoculture plantations, especially oil palm, is simplified due to reduced number of trophic levels.

The most dominant collembolan species at our study sites, Pseudosinella sp.1, occupied a similar trophic niche in each of the land-use systems studied, suggesting that its diet changes little with the conversion of rainforest into plantation systems. Pseudosinella sp.1 colonized both litter and soil, and, as indicated by $\Delta^{15} \mathrm{~N}$ values, occupied the highest trophic position 
among all collembolan species studied. High $\Delta^{15} \mathrm{~N}$ values suggest that this species may either feed on mycrorrhizal fungi, which are enriched in ${ }^{15} \mathrm{~N}$ (Hobbie et al., 2001; Potapov and Tiunov, 2016), and / or live as predators feeding e.g., on nematodes, which are similarly abundant across the land-use systems studied (Krashevska et al., 2019). Conversion of rainforest may have less affected the mineral soil- and root-based resources than litter resources, and this may explain the high abundance of euedaphic Pseudosinella sp.1 in plantations. Similarly, Krause et al. (2019) found the trophic niche of dominant species of oribatid mites to change little with the conversion of rainforest into plantations. This suggests that trophic niches of certain species may be little affected by land-use change despite strong changes in the overall food-web structure.

\section{Trophic differentiation among species}

Variations in $\Delta^{13} \mathrm{C}$ values among collembolan species were more pronounced in rainforest and jungle rubber than in rubber and oil palm plantations. Korotkevich et al. (2018) also found the interspecific (in contrast to intraspecific) variation in trophic niches of Collembola to be higher in natural (forest and meadows) than in disturbed habitats (pastures and lawns). Among the collembolan species studied $\Delta^{13} \mathrm{C}$ values of Pseudosinella sp. 1 in rainforest, and Callyntrura sp.1 and Sphyroteca sp. 1 in jungle rubber differed significantly from those of other collembolan species indicating that these species are able to exploit resources not available to the other collembolan species in the respective land-use system.

Similar to $\Delta^{13} \mathrm{C}$ values, collembolan species in each land-use system, except rubber plantations, also differed in $\Delta^{15} \mathrm{~N}$ values. In jungle rubber Callyntrura sp. 1 was most depleted in $\delta^{15} \mathrm{~N}$ among the studied collembolan species indicating that this species occupied the lowest trophic position pointing to phycophagy (Potapov et al., 2018). In rainforest Pseudosinella sp.1 had the highest $\delta^{15} \mathrm{~N}$ values followed by Isotomiella cf. minor and Acrocyrtus sp. suggesting that these species are as microbivores in undisturbed ecosystems. Overall, based on $\Delta^{13} \mathrm{C}$ and $\Delta^{15} \mathrm{~N}$ values, trophic niche differentiation among species was most pronounced in rainforest, presumably due to the availability of a wider spectrum of food resources and more stable environmental conditions than in plantations. This is likely to result in more efficient food-web functioning in natural ecosystems due to species complementarity (Loreau and Hector 2001), which is partly lost in plantation systems. 


\section{Variations in isotopic niches of Collembola taxa and life forms with land-use systems}

Collembola taxa such as Symphypleona and Paronellidae typically had low $\Delta^{15} \mathrm{~N}$ values, indicating that these taxa feed on algae or lichens (Chahartaghi et al., 2005; Potapov et al., 2018). Symphypleona as well as Paronellidae are well adapted to aboveground life, both are large-sized and possess well-developed visual systems. Such 'atmobiotic' Collembola have been assumed to live at least in part as herbivores on vascular plants or algae (Rusek, 2007). This suggestion is in line with studies from temperate forests investigating variations in stable isotope ratios in Symphypleona (Chahartaghi et al., 2005; Potapov et al., 2016). Paronellidae predominantly occur in tropical regions also living above the ground suggesting that the microhabitat they live in defines feeding preferences of Collembola across different phylogenetic lineages (see life form discussion below). Entomobryidae and Isotomidae occupied high trophic positions and this also is consistent with earlier studies based on variations in stable isotope ratios suggesting that they predominantly feed on microorganisms colonizing decomposing litter materials (Chahartaghi et al., 2005; Potapov et al., 2016). Some species of these two families occupied very high trophic positions resembling those of Onychiuridae and Neanuridae in temperate ecosystems suggesting that at least in part they live as predators or scavengers, presumably including nematodes as prey (Heidemann et al., 2014). Since Onychiuridae and Neanuridae were rare at our study sites, species from other families might have been able to occupy their trophic niches.

Trophic niches of Collembola in our study varied with life form as shown previously for temperate ecosystems (Ponge, 2000; Potapov et al., 2016). Conform to the patterns in collembolan families, the results suggest that atmobiotic and epedaphic species occupied the lowest trophic position across land-use systems, whereas euedaphic species such as Pseudosinella sp.1 occupied the highest trophic position. This is in line with the results of the study of Potapov et al. (2016) indicating that species inhabiting deeper soil layers (hemiedaphic and euedaphic) are more enriched in ${ }^{15} \mathrm{~N}$ than those living in litter and above the ground (epedaphic and atmobiotic). High $\delta^{15} \mathrm{~N}$ values may result from feeding on ectomycorrhizal fungi which are enriched in ${ }^{15} \mathrm{~N}$ (see above; Hobbie et al., 2001; Potapov and Tiunov, 2016), however, this unlikely applies to tropical forests where trees predominantly form mutualistic interactions with arbuscular mycorrhizal fungi. We attributed low $\delta^{15} \mathrm{~N}$ values in epedaphic and atmobiotic Collembola to algae or lichen feeding, which is widespread in Collembola in temperate forests (Potapov et al., 2018), but in our study only few species had $\delta^{15} \mathrm{~N}$ values below those of litter. This contradicts results based on fatty acid analysis suggesting that Collembola feed more on algae in tropical than in temperate ecosystems (Susanti et al., 2019). 
To clarify the contribution of algae in soil food webs in tropical and temperate ecosystems, more data on stable isotope composition of various food resources in tropical forests, or direct experimentation, are needed. Differences between life forms were more pronounced in rainforest and jungle rubber and less in plantation systems, which may reflect the more pronounced litter layer in the former than the latter (Krashevska et al., 2015). Overall, the results suggest that similar to oribatid mites (Tsurikov et al., 2019) the trophic niche structure in Collembola communities in temperate and tropical forests is generally similar and this is partly explained by taxonomic affiliation and life form.

\section{Conclusion}

We showed that the conversion of rainforest into agricultural plantations, such as rubber and oil palm, is associated with changes in trophic niches of Collembola. The use of food resources shifted towards herbivory, with the range of food resources of Collembola in oil palm plantations being the highest, likely due to the heterogeneity in management. By contrast, the range of trophic positions in oil palm plantations was low suggesting that the trophic structure is simplified lacking high but also low trophic levels. This is further supported by the less pronounced trophic niche differentiation among species in monoculture plantations. Similar to the pattern in oribatid mites (Tsurikov et al., 2019), the structure of trophic niches in tropical collembolan communities resembled that in temperate forests. Life form and family identity explained about half of the species-level variation; atmobiotic species occupied the lowest and euedaphic species the highest trophic position, but the difference was less pronounced in plantations. Overall, the results document that changes in community composition associated with the conversion of rainforest into plantation systems are followed by shifts in the trophic structure and trophic niches in collembolan communities, potentially compromising ecosystem functions and food-web stability in plantations.

\section{Acknowledgements}

We thank the State Ministry of Research and Technology of Indonesia (RISTEK), the Indonesian Institute of Sciences (LIPI), Ministry of Forestry (PHKA) and Restoration Ecosystem Indonesia Harapan. We also thank the following persons and organizations: village leaders, local plot owners, PT Humusindo, PT Perkebunan Nusantara VI, Harapan Rainforest, and Bukit Duabelas National Park. We acknowledge support of the Open Access Publication Funds of the University of Göttingen. 


\section{Supplementary Material}

Table S4.1 Model the effect of systems on $\Delta^{13} \mathrm{C}$ isotopic values

Fixed effects:

\begin{tabular}{llllll}
\hline & Estimate & \multicolumn{1}{c}{ Std. Error } & \multicolumn{1}{c}{$d f$} & $\mathrm{t}$ value & $\operatorname{Pr}(>|\mathrm{t}|)$ \\
(Intercept) & 2.95355 & 0.16816 & 65.67466 & 17.563 & $<2 \mathrm{e}-16^{* * *}$ \\
Oil palm & -0.30996 & 0.24381 & 155.96151 & -1.271 & 0.205517 \\
Rainforest & 0.88519 & 0.22810 & 135.10163 & 3.881 & $0.000162 * * *$ \\
Rubber & -0.09414 & 0.21559 & 153.21799 & -0.437 & 0.662969 \\
\hline
\end{tabular}

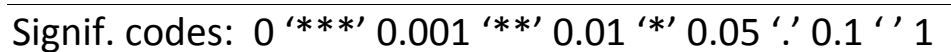

Sum Sq Mean Sq NumDF DenDF F value $\operatorname{Pr}(>\mathrm{F})$

Land.use.System $28.973 \quad 9.6576 \quad 3146.059 .9009 \quad 5.532 \mathrm{e}-06 * * *$

Table S4.2 Significant letter from Tukey-test of the effect of systems on $\Delta^{13} \mathrm{C}$ isotopic value

\begin{tabular}{lcllccc}
\hline Land.use.System & emmean & $\mathrm{SE}$ & $\mathrm{df}$ & lowerCL & upperCL & group \\
\hline Oil palm & 2.73 & 0.187 & 156 & 2.26 & 3.20 & $\mathrm{a}$ \\
Rubber & 2.93 & 0.155 & 156 & 2.54 & 3.32 & $\mathrm{a}$ \\
Jungle rubber & 3.01 & 0.155 & 156 & 2.62 & 3.40 & $\mathrm{a}$ \\
Rainforest & 3.85 & 0.158 & 156 & 3.45 & 4.25 & $\mathrm{~b}$ \\
\hline
\end{tabular}

Confidence level used: 0.95

Table S4.3 Model the effect of systems on $\Delta^{15} \mathrm{~N}$ isotopic values

Fixed effects:

\begin{tabular}{lclcll}
\hline & Estimate & Std. Error & $\mathrm{df}$ & $\mathrm{t}$ value & $\operatorname{Pr}(>|\mathrm{t}|)$ \\
(Intercept) & 4.2145 & 0.5804 & 61.9174 & 7.262 & $7.61 \mathrm{e}-10 * * *$ \\
Oil palm & -1.2019 & 0.7341 & 151.5252 & -1.637 & 0.104 \\
Rainforest & -0.1606 & 0.7130 & 152.8261 & -0.225 & 0.822 \\
Rubber & 0.2538 & 0.6420 & 147.1428 & 0.395 & 0.693 \\
\hline
\end{tabular}

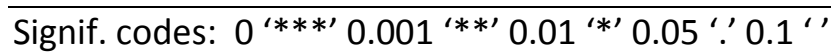

Sum Sq Mean Sq NumDF DenDF Fvalue $\operatorname{Pr}(>\mathrm{F})$

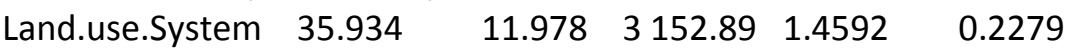

Table S4.4 Significant letter from Tukey-test of the effect of systems on $\Delta^{15} \mathrm{~N}$ isotopic value

\begin{tabular}{lcccccc}
\hline Land.use.System & emmean & $\mathrm{SE}$ & $\mathrm{df}$ & lower $\mathrm{CL}$ & upper $\mathrm{CL}$ & group \\
Oil palm & 4.08 & 0.597 & 156 & 2.58 & 5.58 & $\mathrm{a}$ \\
Jungle rubber & 4.29 & 0.493 & 156 & 3.05 & 5.53 & $\mathrm{a}$ \\
Rainforest & 4.59 & 0.504 & 156 & 3.32 & 5.86 & $\mathrm{a}$ \\
Rubber & 5.03 & 0.493 & 156 & 3.79 & 6.27 & $\mathrm{a}$ \\
\hline
\end{tabular}

Confidence level used: 0.95 
Table S4.5 $\Delta^{13} \mathrm{C}$ isotopic values of species in rainforest

Fixed effects:

\begin{tabular}{lclrrr}
\hline & Estimate & Std. Error & $\mathrm{df}$ & $\mathrm{t}$ value & $\operatorname{Pr}(>|\mathrm{t}|)$ \\
(Intercept) & 3.6396 & 0.26541 & 4.2246 & 13.714 & $1.35 \mathrm{e}-09 * * *$ \\
Isotomiella cf.minor & 0.2470 & 0.2147 & 2.8048 & 1.151 & 0.271 \\
Lepidocyrtus sp.1 & -0.1903 & 0.2277 & 12.7220 & -0.836 & 0.419 \\
Pararrhopalites sp.1 & -0.1412 & 0.1959 & 12.4055 & -0.721 & 0.484 \\
Pseudosinella sp.1 & 0.5791 & 0.2088 & 12.7951 & 2.774 & $0.016 *$ \\
\hline---
\end{tabular}

Signif. codes: 0 “***, 0.001 ، $* *, 0.01$ ، $* 0.05$ ‘, 0.1 ، 1

Type III Analysis of Variance Table with Satterthwaite's method $\begin{array}{lllllll} & \text { Sum Sq Mean Sq NumDF } & \text { DenDF } & \mathrm{F} \text { value } & \operatorname{Pr}(>\mathrm{F}) \\ \text { Species } & 1.7226 & 0.43064 & 4 & 12.539 & 6.3436 & 0.005047\end{array} * *$

$\begin{array}{lllllll}\text { Species } & \text { emmean } & \mathrm{SE} & \mathrm{df} & \text { lower.CL } & \text { upper.CL. } & \text { group } \\ \text { Lepidocyrtus sp.1 } & 3.45 & 0.250 & 12.12 & 2.69 & 4.21 & \mathrm{a} \\ \text { Pararrhopalites sp.1 } & 3.50 & 0.234 & 9.87 & 2.76 & 4.24 & \mathrm{a} \\ \text { Allacma sp.1 } & 3.64 & 0.266 & 14.11 & 2.85 & 4.43 & \mathrm{ab} \\ \text { Isotomiella cf.minor } & 3.89 & 0.240 & 10.75 & 3.14 & 4.63 & \mathrm{ab} \\ \text { Pseudosinella sp.1 } & 4.22 & 0.233 & 9.79 & 3.48 & 4.96 & \mathrm{~b}\end{array}$

Table S4.6 $\Delta^{13} \mathrm{C}$ isotopic values of species in jungle rubber

Fixed effects:

\begin{tabular}{lrrrrl}
\hline & Estimate & Std. Error & df & t value & $\operatorname{Pr}(>|\mathrm{t}|)$ \\
(Intercept) & 3.119551 & 0.250028 & 12.850165 & 12.477 & $1.49 \mathrm{e}-08^{* * *}$ \\
Callyntrura sp.1 & -0.689469 & 0.264358 & 16.913427 & -2.608 & $0.0184^{*}$ \\
Folsomides centralis & 0.007335 & 0.216371 & 16.764134 & 0.034 & 0.9734 \\
Homidia cingula & 0.315800 & 0.246299 & 16.202993 & 1.282 & 0.2178 \\
Pseudosinella sp.1 & 0.024740 & 0.233050 & 16.270388 & 0.106 & 0.9168 \\
Spyrhoteca sp.1 & -0.700629 & 0.282540 & 16.649945 & -2.480 & $0.0242 *$ \\
\hline--
\end{tabular}

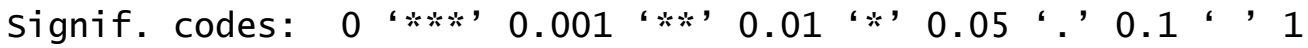

Type III Analysis of Variance Table with Satterthwaite's method

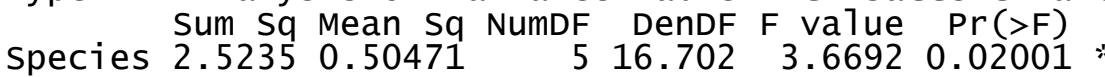

$\begin{array}{lcccccc}\text { Species } & \text { emmean } & \text { SE } & \text { df } & \text { lower.CL } & \text { upper.CL } & \text { group } \\ \text { Spyrhoteca sp.1 } & 2.42 & 0.307 & 19.7 & 1.52 & 3.31 & \text { ab } \\ \text { Callyntrura sp.1 } & 2.43 & 0.291 & 17.9 & 1.57 & 3.29 & \mathrm{a} \\ \text { Ascocyrtus cinctus } & 3.12 & 0.251 & 13.0 & 2.34 & 3.90 & \mathrm{ab} \\ \text { Folsomides centralis } & 3.13 & 0.240 & 11.7 & 2.37 & 3.89 & \mathrm{ab} \\ \text { Pseudosinella sp.1 } & 3.14 & 0.263 & 14.7 & 2.35 & 3.94 & \mathrm{ab} \\ \text { Homidia cingula } & 3.44 & 0.280 & 17.0 & 2.60 & 4.27 & \mathrm{~b}\end{array}$


Table S4.7 $\Delta^{13} \mathrm{C}$ isotopic values of species in rubber plantations

Fixed effects:

\begin{tabular}{|c|c|c|c|c|c|c|}
\hline \multirow{3}{*}{$\begin{array}{l}\text { (Intercept) } \\
\text { Alloscopus tetracanthus }\end{array}$} & Estimate & \multirow{2}{*}{$\begin{array}{l}\text { Std. Error } \\
0.3767\end{array}$} & \multicolumn{2}{|c|}{$\mathrm{df} \quad \mathrm{t}$ value } & \multicolumn{2}{|c|}{$\operatorname{Pr}(>|t|)$} \\
\hline & 2.2822 & & 23.6322 & 6.059 & \multicolumn{2}{|c|}{$3.14 \mathrm{e}-06 * * *$} \\
\hline & s 0.9751 & 0.4256 & 19.5089 & 2.291 & \multicolumn{2}{|c|}{$0.0332 *$} \\
\hline Ascocyrtus cinctus & 0.6071 & 0.4268 & 19.6045 & 1.423 & \multicolumn{2}{|c|}{0.1706} \\
\hline Folsomides centralis & 0.7385 & 0.4127 & 18.6598 & 1.789 & \multicolumn{2}{|c|}{0.0898 . } \\
\hline Homidia cingula & 0.7013 & 0.4151 & 18.9387 & 1.689 & \multicolumn{2}{|c|}{0.1075} \\
\hline Pseudosinella sp.1 & 0.9245 & 0.3890 & 18.9302 & 2.377 & \multicolumn{2}{|c|}{$0.0282 *$} \\
\hline \multicolumn{7}{|c|}{ Signif. codes: 0 “***, $0.001 ، * *, 0.01 ، *, 0.05 ،, 0.1 ،, 1$} \\
\hline \multicolumn{7}{|c|}{ 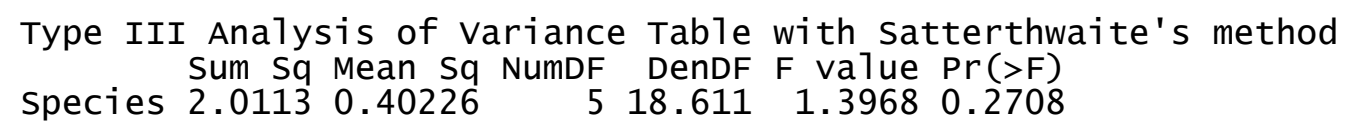 } \\
\hline Species & emmean & SE df & lower.C & & upper.CL & group \\
\hline Acrocyrtus sp.1 & 2.28 & 0.38323 .6 & 1.18 & & 3.38 & a \\
\hline Ascocyrtus cinctus & 2.89 & 0.31120 .2 & 1.98 & & 3.80 & $a$ \\
\hline Homidia cingula & 2.98 & 0.31120 .3 & 2.08 & & 3.89 & a \\
\hline Folsomides centralis & 3.02 & 0.31120 .3 & 2.12 & & 3.93 & a \\
\hline Pseudosinella sp.1 & 3.21 & 0.27116 .8 & 2.40 & & 4.01 & a \\
\hline Alloscopus tetracanthus & 3.26 & 0.31120 .2 & 2.35 & & 4.16 & a \\
\hline
\end{tabular}

Table S4.8 $\Delta^{13} \mathrm{C}$ isotopic values of species in oil palm plantations

Fixed effects:

\begin{tabular}{|c|c|c|c|c|c|}
\hline & Estimate & Std. Error & $d f$ & $\mathrm{t}$ value & $\operatorname{Pr}(>|t|)$ \\
\hline (Intercept) & 2.9032 & 0.6120 & 9.2615 & 4.744 & $0.000973 * * *$ \\
\hline Isotomiella cf.minor & -0.3096 & 0.4321 & 8.8038 & -0.717 & 0.492224 \\
\hline Pseudosinella sp.1 & 0.2398 & 0.3106 & 8.2876 & 0.772 & 0.461456 \\
\hline
\end{tabular}

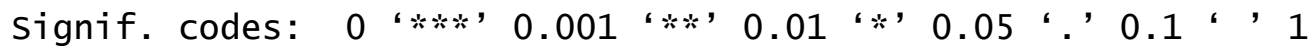

Type III Analysis of Variance Table with Satterthwaite's method Sum Sq Mean Sq NumDF DenDF $F$ value $\operatorname{Pr}(>F)$

$\begin{array}{lllllll}\text { Species } & 0.62334 & 0.31167 & 2 & 8.59 & 1.1034 & 0.3745\end{array}$

$\begin{array}{lcccccc}\text { Species } & \text { emmean } & \text { SE df } & \text { lower.CL } & \text { upper.CL } & \text { group } \\ \text { Isotomiella cf.minor } & 2.59 & 0.634 & 10.01 & 0.781 & 4.41 & \text { a } \\ \text { Folsomides centralis } & 2.90 & 0.613 & 9.11 & 1.115 & 4.69 & \text { a } \\ \text { Pseudosinella sp.1 } & 3.14 & 0.584 & 7.70 & 1.371 & 4.91 & \text { a }\end{array}$


Table S4.9 $\Delta^{15} \mathrm{~N}$ isotopic values of species in rainforest

Fixed effects:

\begin{tabular}{|c|c|c|c|c|c|}
\hline & Estimate & Std. Error & $\mathrm{df}$ & t value & $\operatorname{Pr}(>|t|)$ \\
\hline (Intercept) & 0.5851 & 1.8664 & 18.9925 & 0.313 & 0.75734 \\
\hline Isotomiella cf.minor & 5.0897 & 2.1449 & 14.7976 & 2.373 & $0.03165 *$ \\
\hline Lepidocyrtus sp.1 & 3.8540 & 2.2794 & 14.9412 & 1.691 & 0.11162 \\
\hline Pararrhopalites sp.1 & 2.4453 & 2.0135 & 13.3735 & 1.214 & 0.24558 \\
\hline Pseudosinella sp.1 & 8.2953 & 2.0858 & 14.8565 & 3.977 & $0.00124 * *$ \\
\hline \multirow{2}{*}{\multicolumn{6}{|c|}{ 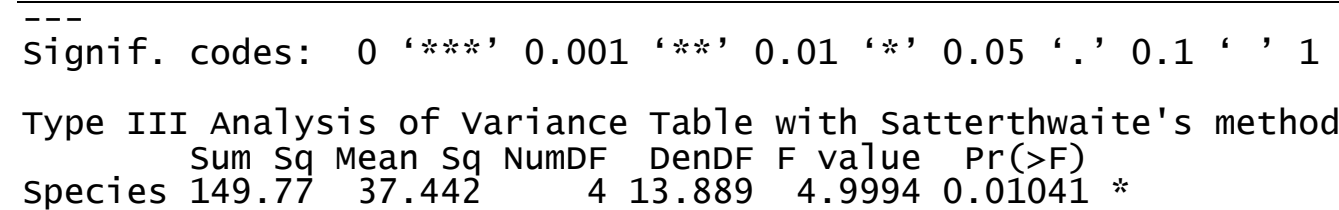 }} \\
\hline & & & & & \\
\hline Species & emmean & SE df & lower.CL & upper.CL & group \\
\hline Allacma sp.1 & 0.585 & 1.9319 .0 & -4.906 & 6.08 & a \\
\hline Pararrhopalites sp.1 & 3.030 & 1.4117 .0 & -1.041 & 7.10 & $a$ \\
\hline Lepidocyrtus sp.1 & 4.439 & 1.6918 .8 & -0.377 & 9.26 & $a b$ \\
\hline Isotomiella cf.minor & 5.675 & 1.5218 .1 & 1.315 & 10.03 & $a b$ \\
\hline Pseudosinella sp.1 & 8.880 & 1.4017 .1 & 4.838 & 12.92 & $b$ \\
\hline
\end{tabular}

Table S4.10 $\Delta^{15} \mathrm{~N}$ isotopic values of species in jungle rubber

Fixed effects:

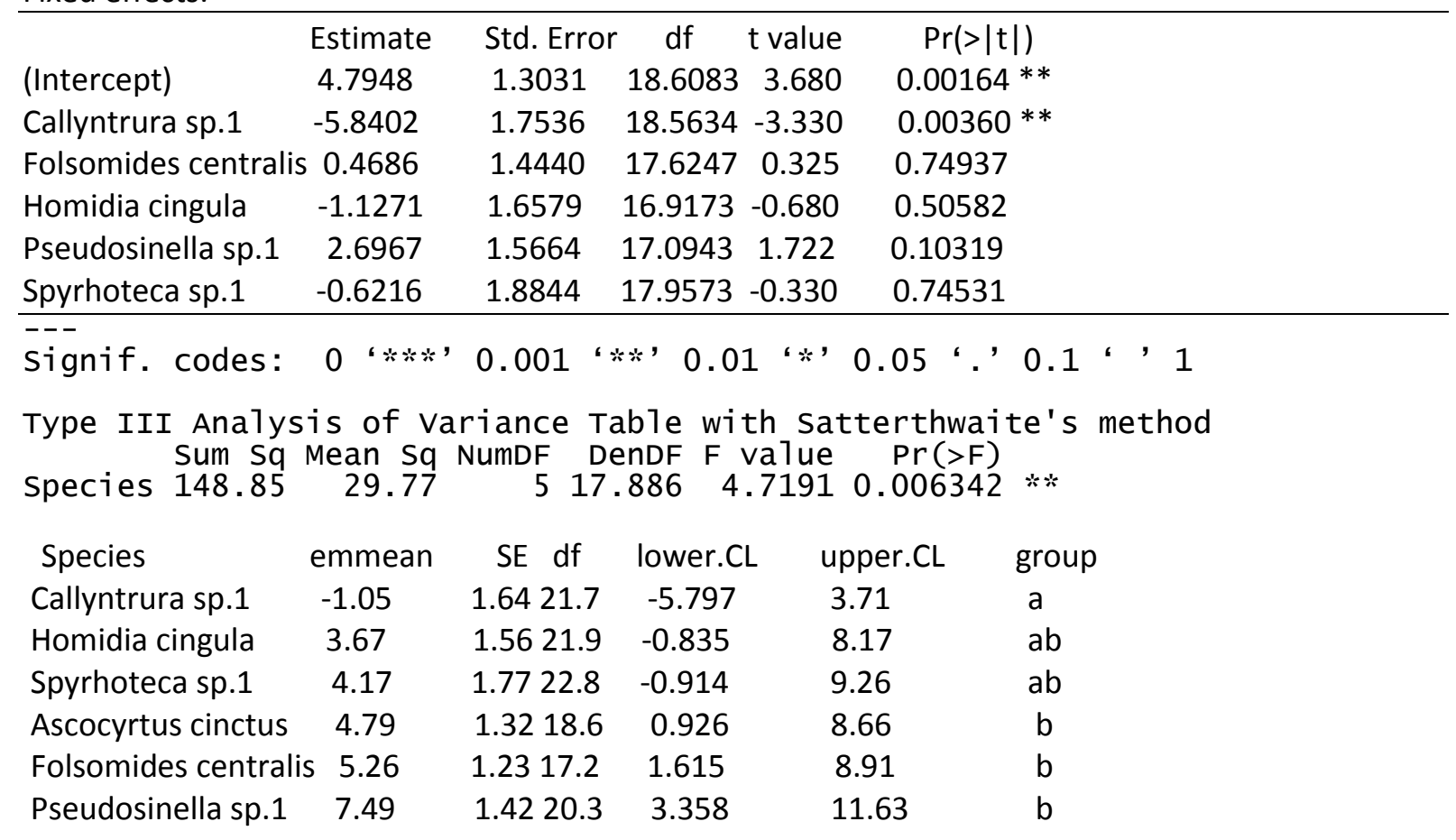


Table S4.11 $\Delta^{15} \mathrm{~N}$ isotopic values of species in rubber plantations

Fixed effects:

\begin{tabular}{|c|c|c|c|c|c|}
\hline & Estimate & Std. Erro & $\partial r \quad d f$ & t value & $\operatorname{Pr}(>|t|)$ \\
\hline (Intercept) & 8.249 & 1.306 & 24.000 & 6.315 & $1.58 \mathrm{e}-06 * * *$ \\
\hline Alloscopus tetracanthus & -2.920 & 1.577 & 21.025 & -1.852 & 0.0781 . \\
\hline Ascocyrtus cinctus & -4.205 & 1.579 & 21.161 & -2.662 & $0.0145 *$ \\
\hline Folsomides centralis & -3.798 & 1.544 & 19.753 & -2.460 & $0.0232 *$ \\
\hline Homidia cingula & -4.357 & 1.548 & 20.087 & -2.814 & $0.0107 *$ \\
\hline Pseudosinella sp.1 & -1.799 & 1.453 & 19.802 & -1.238 & 0.2300 \\
\hline \multicolumn{6}{|c|}{ Signif. codes: $0 ، * * *, 0.001 ، * *, 0.01 ، * 0.05 ،, 0.1 ،, 1$} \\
\hline \multicolumn{6}{|c|}{ 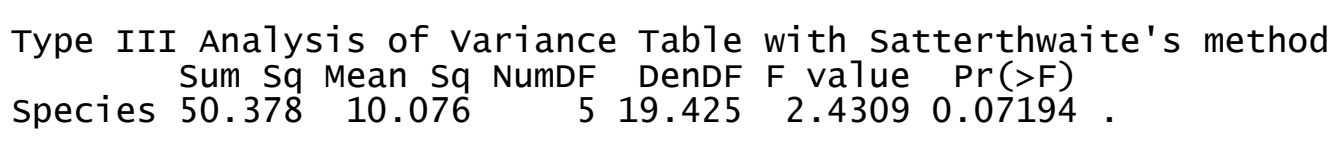 } \\
\hline Species & emmean & SE df & lower.CL & upper.CL & group \\
\hline Homidia cingula & 3.89 & 1.05023 .1 & 0.874 & 6.91 & a \\
\hline Ascocyrtus cinctus & 4.04 & 1.05023 .1 & 1.026 & 7.06 & a \\
\hline Folsomides centralis & 4.45 & 1.04823 .1 & 1.437 & 7.47 & a \\
\hline Alloscopus tetracanthus & s 5.33 & 1.04923 .1 & 2.314 & 8.34 & a \\
\hline Pseudosinella sp.1 & 6.45 & 0.88721 .5 & 3.881 & 9.02 & a \\
\hline Acrocyrtus sp.1 & 8.25 & 1.34424 .0 & 4.396 & 12.10 & a \\
\hline
\end{tabular}

Table S4.12 $\Delta^{15} \mathrm{~N}$ isotopic values of species in oil palm plantations

Fixed effects:

\begin{tabular}{lrlrll}
\hline & Estimate & \multicolumn{1}{l}{ Std. Error } & \multicolumn{1}{c}{ df } & $\mathrm{t}$ value & \multicolumn{1}{c}{$\operatorname{Pr}(>|\mathrm{t}|)$} \\
(Intercept) & 3.5453 & 0.4383 & 11.6928 & 8.089 & $3.97 \mathrm{e}-06^{* * *}$ \\
Isotomiella cf.minor & 0.4392 & 0.4349 & 9.8314 & 1.010 & 0.336792 \\
Pseudosinella sp.1 & 2.0362 & 0.3194 & 8.6821 & 6.375 & $0.000151 * * *$ \\
\hline
\end{tabular}

Signif. codes: 0 “***, 0.001 “**, 0.01 “* 0.05 ‘' 0.1 “' 1

Type III Analysis of Variance Table with Satterthwaite's method

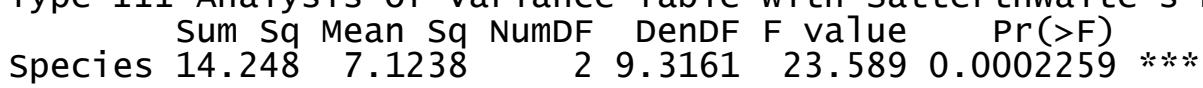

$\begin{array}{lcccccc}\text { Species } & \text { emmean } & \text { SE df } & \text { lower.CL } & \text { upper.CL } & \text { group } \\ \text { Folsomides centralis } & 3.55 & 0.44311 .4 & 2.31 & 4.78 & \mathrm{a} \\ \text { Isotomiella cf.minor } & 3.98 & 0.47112 .8 & 2.69 & 5.28 & \mathrm{a} \\ \text { Pseudosinella sp.1 } & 5.58 & 0.398 & 8.6 & 2.41 & 6.76 & \mathrm{~b}\end{array}$


Chapter 4

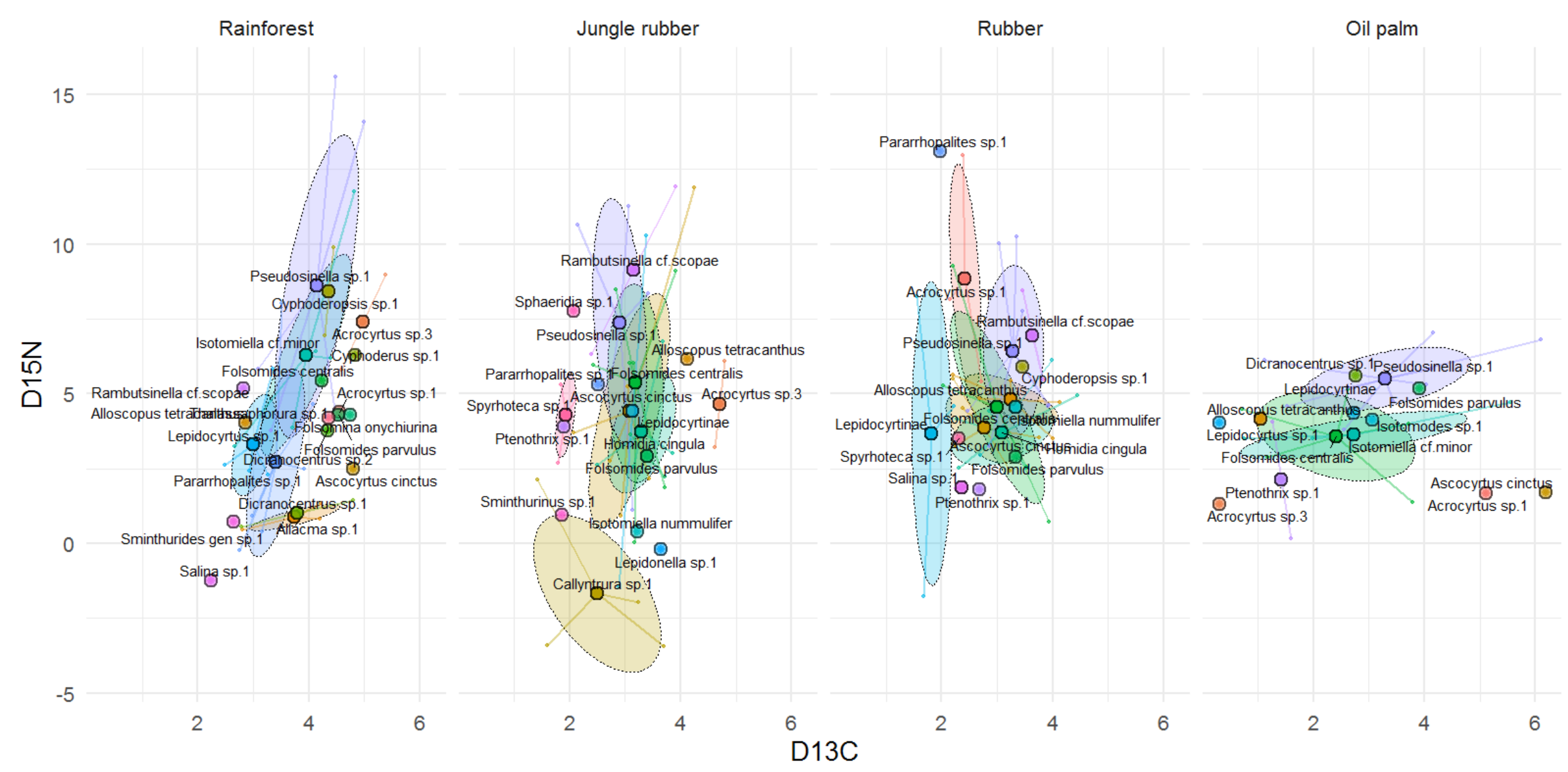

Supplementary Material Figure 4.1. Isotopic niche of Collembola species in different land-use systems (rainforest, jungle rubber, rubber and oil palm plantations). Ellipses denote $60 \%$ confidence intervals and different species are shown with colours. Large coloured dots represent means of land-use systems, small points represent individual measurements. 


\section{CHAPTER 5}

\section{SEASONAL FLUCTUATIONS OF LITTER AND SOIL COLLEMBOLA AND THEIR DRIVERS IN RAINFOREST AND PLANTATIONS}

Winda Ika Susanti, Rahayu Widyastuti, Stefan Scheu, Anton Potapov
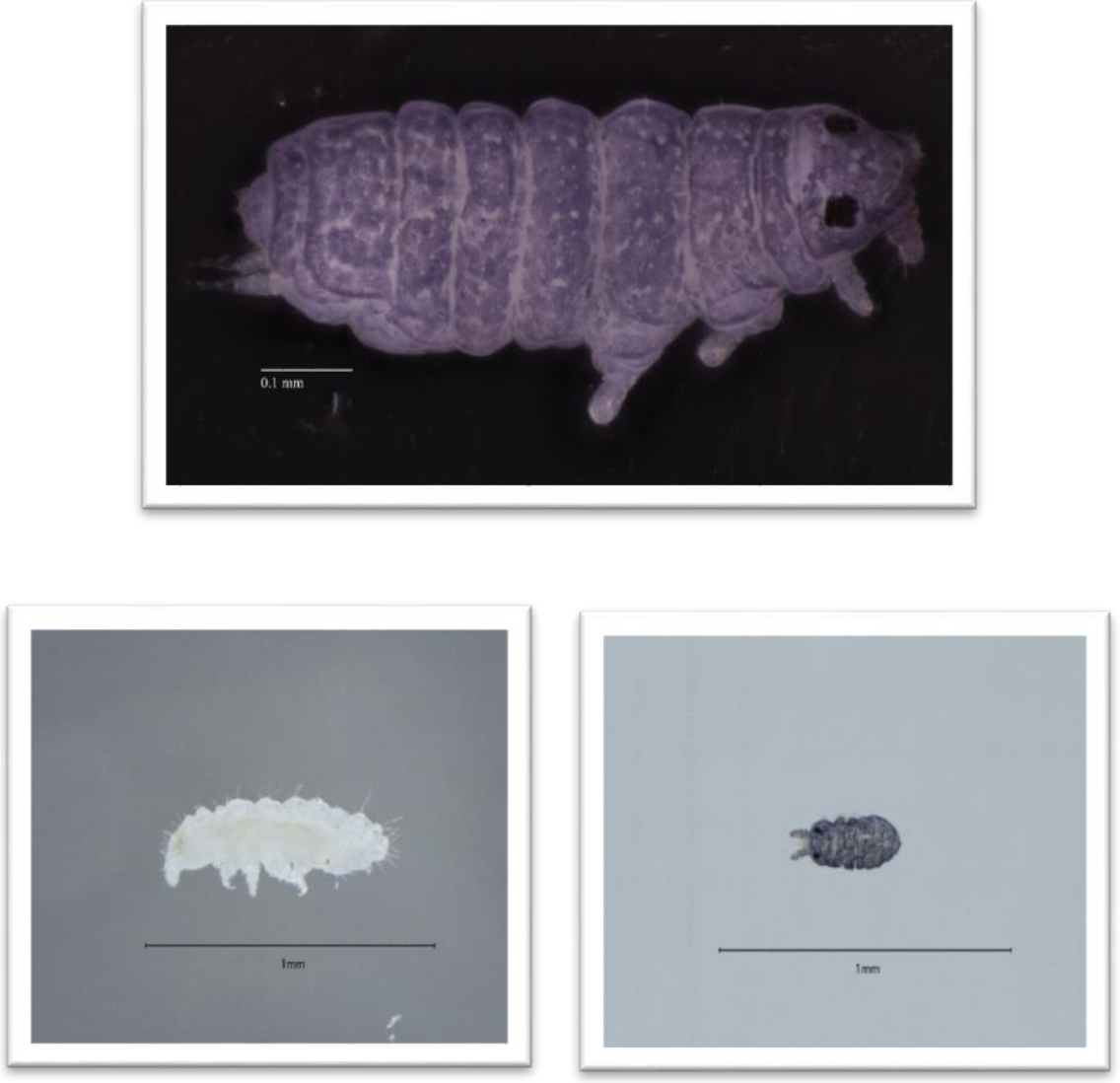

Photos by Winda Ika Susanti 


\title{
SEASONAL FLUCTUATIONS OF LITTER AND SOIL COLLEMBOLA AND THEIR DRIVERS IN RAINFOREST AND PLANTATIONS
}

\begin{abstract}
Collembola are among the most abundant and diverse mesofauna that affect decomposition, control microbial functioning and support arthropod predators. Rainforest conversion and expansion of plantations in tropical regions are associated with biodiversity decline and also changes in microclimate, but information on the impact of tropical land use on seasonal fluctuations of Collembola communities is very limited. We investigated seasonal fluctuations in density and community composition of Collembola in rainforest, rubber and oil palm plantations in Jambi province (Sumatra, Indonesia), a region with moderate seasonality (wet and dry seasons) that experienced one of the strongest deforestation globally during the last decades. Collembola density in the litter layer, but not in soil, was at a maximum at the beginning of the wet season. Euedaphic and hemiedaphic species, living in the lower litter layers and soil, fluctuated less with season than epedaphic and atmobiotic species, living in upper litter layers. Collembola community composition changed with season in all land-use systems, with the differences being more pronounced in the litter than in the soil layer. Differences in community composition among land-use systems were most pronounced at the beginning of the dry season (June). Both in litter and soil, water content was identified as main factor related to seasonal variations in species composition of Collembola in rainforest in the wet season, whereas $\mathrm{pH}$ was identified as main factor of species composition in the wet season of plantation systems. Mycorrhizal and fungal markers were identified as important factors for community composition in the wet season of litter in plantation systems, whereas Gram-positive and Gram-negative bacteria correlated with Collembola community composition in the dry season in soil of rainforest. In addition, soil $\mathrm{C} / \mathrm{N}$ ratio was identified as important factor driving Collembola community composition in rainforest. Overall, the results show that seasonal fluctuations of Collembola communities in general were similar in each of the land-use systems, indicating that they are driven by common enironmental factors. However, fluctuations in Collembola communities were stronger in microhabitats less buffered against environmental variations (litter versus soil)
\end{abstract}




\section{Chapter 5}

and the effect of plantations on Collembola communities was most pronounced in the dry season reflecting complemetary negative effects of decreased moisture and land use on tropical soil communities.

Keywords: springtail, community structure, seasonal fluctuations, rainforest conversion, plantation

\section{Introduction}

Rainforest conversion into agricultural plantations in Indonesia increased greatly in the last 30 years and this is predicted to continue (Koh and Ghazoul, 2010; Gatto et al., 2015). Lowland rainforest in Jambi province, Sumatra, has been converted in large into oil palm (16\% of total area) and rubber plantations (12\%) (Gatto et al., 2015). Therefore, Jambi province represents a model region to investigate the effect of rainforest conversion on biodiversity and ecosystem functioning at local and regional scales (Clough et al., 2016; Drescher et al., 2016). The conversion of tropical rainforest into plantations is associated with the degradation and destruction of habitats (Titeux et al., 2016), resulting in the loss of plant and animal biodiversity above the ground (Fitzherbert et al., 2008; Clough et al., 2016), but increasingly this is also documented for belowground taxa including nematodes (Krashevska et al., 2019), testate amoebae (Krashevska et al., 2016), spiders (Potapov et al., 2020b) and litter macroinvertebrates (Barnes et al., 2014). These changes are likely to be associated with changes in ecosystem functioning (Sousa et al., 2006; Fitzherbert et al., 2008; Gilbert, 2012; Barnes et al., 2014; Clough et al., 2016). Conversion of forest into other landuse systems, especially into monoculture plantations, is associated with changes in microclimate, particularly air temperature, relative humidity, vapour pressure deficit and soil temperature, being driven in large by canopy openness (Meijide et al., 2018). Forests are cooler than oil palm and rubber monocultures, while the latter are drier and have higher vapour pressure deficit. These microclimatic changes with rainforest conversion are likely modifying seasonal changes in ecological communities, but this remains to be explored for belowground communities.

Collembola are a dominant group of soil animals globally, being particularly abundant in forest soils (Hopkin, 1997; Rusek, 1998; Devi et al., 2011). They significantly 


\section{Chapter 5}

affect soil microbial communities, nutrient cycling and soil fertility by feeding on soil microorganisms and dead organic matter (Rusek, 1998; Coulibaly et al., 2019). In temperate forests, land-use and management practices have been shown to affect microbial community composition and the relative strength of the fungal and bacterial energy channel (Pollierer et al., 2015) as well as impacting Collembola density, diversity and community composition (Deharveng 1996; Filser et al., 2002; Widrializa et al., 2015). Density and community composition of Collembola in soil and litter may follow seasonal variations in microclimate throughout the year. This has been shown e.g., for Collembola and Acari in litter and soil in South Australian forests (Hutson and Veitch, 1987), Collembola biomass and abundance in oak forests in Switzerland (Xu et al., 2012). In the tropics with less pronounced seasonal climatic variations than in high latitude ecosystems, seasonal fluctuations in Collembola density and community composition also have been reported, with usually lower density in the dry than in the wet season (Palacios-Vargas and Castaño-Meneses, 2003; Wiwatwitaya and Takeda, 2005; Muturi et al., 2009). Similarly, Mayvan et al. (2015) reported Collembola density in temperate forests (Iran) to be lowest in the dry season. However, little is known on changes in seasonal variations in Collembola communities due to the conversion of forest into agricultural plantation systems, particularly in the tropics.

Community composition of litter and soil Collembola is linked to both biotic and abiotic factors. Salamon et al. (2008) found dominant functional groups/species of Collembola to depend mainly on local abiotic factors such as $\mathrm{pH}$ and soil water content. This is in line with results of the study of Susanti et al. (2021) that $\mathrm{pH}$ and water content strongly affect Collembola communities in rainforest and agricultural replacement systems in Indonesia. Pollierer and Scheu (2017), however, assumed the detrimental effect of low pH to be related to reduced abundance of microorganisms as major food resource. Soil $\mathrm{pH}$ also has been identified as important factor driving Collembola communities in contaminated soil (Errington et al., 2018), but the effect also might be indirect via changes in the availability of microorganisms as food resource (Filser et al., 2002). Petersen (2011) reported Collembola community composition to depend on temperature and moisture with these factors likely affecting Collembola directly. Both biotic and abiotic factors change following changes in land-use and this likely results in changes in seasonal variations in Collembola community composition. However, information on effects of the conversion of rainforest into 


\section{Chapter 5}

monoculture plantations on seasonal variations of soil animal communities, such as Collembola, is lacking.

Seasonal variations in Collembola communities are likely to be linked closely to their vertical stratification in soil. It is well known that Collembola community composition varies between soil layers and this is closely linked to changes in the abundance of Collembola of different life forms (Salmon et al., 2014). Some studies reported euedaphic species to be less sensitive to environmental and seasonal variations than hemi-and epedaphic species (Chauvat et al., 2003; Bokhorst et al., 2012). In fact, epedaphic Collembola species in sugar maple forests in Canada (Chagnon et al., 2000) were shown to be more strongly affected by seasonal changes than euedaphic species.

In this study, we investigated species composition of Collembola communities in litter and soil in rainforest, rubber and oil palm plantations in Jambi province, Sumatra, Indonesia. To explore seasonal fluctuations, samples were taken at four sampling dates in 2017 varying in temperature and humidity. Based on previous studies on the impact of landuse change on soil invertebrate communities, we hypothesized that (1) Collembola density in litter and soil changes with season with Collembola density being lowest in the dry season, (2) the vertical stratification of soil and litter Collembola changes with season with this being less pronounced in euedaphic than in epedaphic and hemiedaphic species, (3) differences in the community composition of Collembola between rainforest and plantation systems will be most pronounced in the dry season, and (4) $\mathrm{pH}$ and water content are the most important factors correlating with Collembola community composition across seasons.

\section{Material and Methods}

\section{Study sites}

The study was conducted in the framework of the EFForTS project investigating in a comprehensive way ecological and socioeconomic changes associated with the transformation of rainforest into plantation systems (http://www.unigoettingen.de/en/310995.html). Soil and litter samples were taken in lowland rainforest, rubber (Hevea brasiliensis) plantations and oil palm (Elaeis guineensis) plantations, located in Jambi province, southwest Sumatra, Indonesia. The climate is tropical and humid with a rainy season from October to April and a dryer period from June to September (Drescher et 


\section{Chapter 5}

al., 2016). The study sites were located at similar altitude varying from 50 to $100 \mathrm{~m}$ a.s.l.; the overall area where sampling sites were distributed was about $80 \mathrm{~km}$ in diameter with the distance between sites being about $70 \mathrm{~km}$ (for more details see Drescher et al., 2016). Rainforest was included to represent baseline conditions allowing to evaluate changes due to the conversion into agricultural plantations.

Rubber and oil palm plantations were intensively managed monocultures of 6 to 16 and 8 to 15 years, respectively (Drescher et al., 2016). Typically, oil palm plantations were established after clearing and burning of jungle rubber, whereas rubber plantations were established after logging of rainforest (Allen et al., 2015). Soils in the Harapan region mainly comprise loamy Acrisols with low fertility (Allen et al., 2015; Kotowska et al., 2015). Oil palm plantations were fertilized once in the rainy season and once in the dry season with NPK complete fertilizer (i.e., Phonska and Mahkota), potassium chloride $(\mathrm{KCl})$ and urea $\left(\mathrm{CO}\left(\mathrm{NH}_{2}\right)_{2}\right)$. Fertilizer addition to oil palm plantations was in the range of $300-550 \mathrm{~kg} \mathrm{NPK}$ fertilizer ha $\mathrm{y}^{-1} \mathrm{y}^{-1}$. Manual and chemical weeding took place throughout the year in both rubber and oil palm plantations. The most commonly used herbicides were glyphosate and paraquat, applied at an average rate of 2-5 $\mathrm{L} \mathrm{ha}^{-1} \mathrm{y}^{-1}$ (Allen et al., 2015; Kotowska et al., 2015; Clough et al., 2016). More details on management practices of the studied smallholder monoculture plantations are described in Allen et al. (2015).

\section{Sampling procedure}

Samples were taken in 2017 at four different sampling dates: end of the wet season (March), beginning of the dry season (June), end of the dry season (August), and beginning of the wet season (November). Detailed climate data for each season is given in Table 5.1. Samples were taken within $50 \mathrm{~m}$ x $50 \mathrm{~m}$ plots established at each study site (see Drescher $e t$ $a l ., 2016$ ) with minimum distance of $300 \mathrm{~m}$, but usually more than $1 \mathrm{~km}$ between plots. Each land-use system was replicated four times resulting in a total of 12 plots (3 land-use systems $\mathrm{x} 4$ replicates). From each plot at each sampling date one randomly positioned sample was taken resulting in 48 soil cores in total. Each sample measured 16 x $16 \mathrm{~cm}$ taken to a depth

of $5 \mathrm{~cm}$ of the mineral soil. Litter and soil were separated in the field and processed separately (96 samples in total). Then, samples were transported to the laboratory for extraction of soil animals. Animals were extracted by heat for three days (Kempson et al., 1963) and stored in 


\section{Chapter 5}

$70 \%$ ethanol until further processing. Environmental variables were measured in composite samples of each litter and soil (five cores per plot within a radius of ca. $2 \mathrm{~m}$ around the soil animal sample), including abiotic factors ( $\mathrm{pH}$, water content, $\mathrm{C}: \mathrm{N}$ ratio) and biotic factors (microbial community composition in litter and soil as indicated by phospholipid fatty acids; Krashevska et al., 2015, V. Krashevska unpubl. data) including the sum of phospholipid fatty acid (PLFA) marker lipids of Gram-positive bacteria (i15:0, a15:0, i16:0, i17:0), Gram-

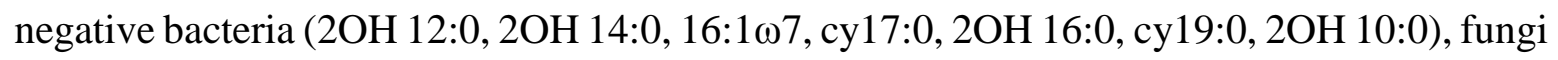
$(18: 2 \omega 6,9)$, algae (20:5 13$)$, and arbuscular mycorrhizal fungi based on the neutral lipid fatty acid (NLFA) 16:1 $105 \mathrm{c}$. Litter and soil $\mathrm{pH}\left(\mathrm{CaCl}_{2}\right)$ was measured using a digital $\mathrm{pH}$ meter. Aliquots of litter and soil material were dried at $65^{\circ} \mathrm{C}$ for $72 \mathrm{~h}$, milled and analyzed for total $\mathrm{C}$ and $\mathrm{N}$ concentrations using an elemental analyzer (Carlo Erba, Milan, Italy). Water content of litter and soil were determined gravimetrically. Details on environmental factors are presented in Appendix 5.1 (litter) and Appendix 5.2 (soil).

Table 5.1. Climate data used in this study for each land-use system rainforest, rubber plantation, oil palm plantation) and sampling time (season; March, June, August, November). Monthly means of daily average measurements are given.

\begin{tabular}{lllll}
\hline $\begin{array}{l}\text { Climate } \\
\text { variable }\end{array}$ & $\begin{array}{l}\text { Sampling } \\
\text { time }\end{array}$ & Rainforest & Rubber & Oil palm \\
\hline Air Humidity & March & 98.4 & 92.2 & 91.2 \\
& June & 97.7 & 93.2 & 91.5 \\
& August & 97.5 & 83.2 & 88.7 \\
& November & 98.5 & 93.4 & 91.5 \\
\hline Air Temperature & March & 24.0 & 26.0 & 24.6 \\
& June & 24.3 & 25.6 & 26.0 \\
& August & 24.1 & 25.4 & 25.8 \\
& November & 24.2 & 25.5 & 26.1 \\
\hline Soil Moisture & March & 34.3 & N/A & 34.5 \\
& June & 31.3 & 40 & 34.8 \\
& August & 31.1 & 30.4 & 30.4 \\
& November & 34.2 & 33.1 & 33.6 \\
\hline Soil Temperature & March & 25.3 & N/A & 27.8 \\
& June & 25.2 & 26.1 & 27.7 \\
& August & 25.0 & 25.7 & 27.8 \\
& November & 25.5 & 25.3 & 28.3 \\
\hline
\end{tabular}




\section{Chapter 5}

\section{Species identification}

Collembola were sorted into morphogroups under a dissecting microscope (Stemi 508, Zeiss, Jena, Germany) at 50x magnification based on basic morphological characters (body shape, morphology of furca, antennae, and number of eyes). Several individuals of each morphogroup from each sample were subsequently cleared with Nesbitt solution on a heating plate $\left(50^{\circ} \mathrm{C}\right)$ for $3-10 \mathrm{~min}$. Then, the animals were mounted on slides with Hoyer's solution (for details see Glime and Wagner, 2017). Collembola were identified to species level using a compound microscope (Axiovert 35, Zeiss) at maximum 400x magnification, using the checklist for Indonesian Collembola (Suhardjono et al., 2012) and additional articles containing keys for Collembola of southeast Asia, particularly Indonesia (Potapov and Starostenko, 2002; Potapov, 2012; Mateos and Greenslade, 2015). Due to the poorly described fauna, in many cases we had to assign individuals to morphospecies without Latin binomials (in total $70 \%$ of all identified species). Below, we refer to all identifications as 'species' for simplicity. Whenever possible, juvenile specimens were ascribed to species by comparing with adults or subadults. To indicate vertical stratification, Collembola were classified into life forms. Four life form groups were distinguished based on Rusek (2007):

- Atmobiotic forms - length up to 8-10 mm, brightly and often motley colored, long limbs and full set of ocelli, body shape either round or elongated; mostly inhabiting macrophytes such as grasses, bushes, trunks and branches of trees, but also the litter surface.

- Epedaphic forms - strong pigmentation, fully developed furca and appendages, complete set of pigmented eyes $(8+8)$, medium or large size, pronounced but frequently uniform coloring; typically colonizing the litter layer.

- Hemiedaphic forms - reduction of body pigmentation and eye numbers (and/or eyes' pigmentation), poorly developed furca, medium or small size; inhabiting partly decomposed litter or rotten wood.

- Euedaphic forms - absence of pigmentation and eyes, often also furca reduced, typically elongated soft body of medium or small size; largely inhabiting the upper mineral layers of the soil (humus horizon). 


\section{Chapter 5}

\section{Statistical analysis}

In all analyses, we used individual soil samples as replicates (litter and soil separately), i.e. $\mathrm{n}=96$ (4 sampling dates x 3 land-use systems x 4 replicates x 2 layers). Statistical analyses were conducted using R version 3.5.3 (R Core Team, 2017). We inspected the effect of land-use system (rainforest, rubber, oil palm), layer (litter, soil), and sampling date (season) as well as their interactions on the abundance of Collembola. Generalized linear models with these factors were run using glmer.nb (negative binomial distribution) in the lme4 package v. 1.1-21 (Bates et al., 2015). Soil core was included as random effect to account for interdependency of soil and litter samples of the same soil core. The same analysis was applied in each of the life forms separately. Statistical significances of the factors were evaluated using Anova in car package

We applied linear discriminant analyses (LDA) to assess differences in community composition of Collembola species among land-use systems at each sampling date. Only species occurring in at least three plots were included in the analysis. Non-metric multidimensional scaling (metaMDS) with six dimensions was done before continuing with LDA. LDA was done using the MASS package including all six axes from the NMDS. Wilks Lambda and $p$-values were used for inspecting the effect of sampling date and land-use system on community composition, and were calculated using manova in the pander package v. 0.6.3 (Daroczi and Tsegelskyi, 2018). Pairwise tests between land-use systems were conducted using HotellingsT2 in the ICSNP package v. 1.1-1 (Nordhausen et al., 2018). To numerically estimate distances among communities of different land-use systems squared Mahalanobis distances $\left(\mathrm{MD}^{2}\right.$ ) between land-use systems were calculated using the mahal function in the HDMD package v. 1.2 (McFerrin, 2013).

Multivariate community analyses (canonical correspondence analysis; CCA) was used to identify environmental factors associated with community composition of Collembola across different land-use systems and sampling dates. We applied forwardselection CCA as implemented in CANOCO 5.02 (ter Braak and Smilauer, 2012). Sampling dates and land-use systems were included as silent variables not affecting the ordination. CCAs were performed because the lengths of gradients were 3.6 SD units for litter and 3.5 SD units for soil indicating unimodal species - environment relationships (Leaps, 2003). Only species occurring in at least three plots were included in the analyses (Appendix 5.3 and 5.4). 


\section{Chapter 5}

The following environmental variables were included in the CCA that were a priori assumed to affect Collembola community composition: carbon-to-nitrogen ratio, $\mathrm{pH}$ value $\left(\mathrm{CaCl}_{2}\right)$, water content (\%), sum of PLFA marker lipids of Gram-positive bacteria and Gram-negative bacteria, fungal PLFA marker, relative PLFA marker of algae, relative marker of arbuscular mycorrhizal fungi based on the neutral lipid fatty acid (NLFA) (Krashevska et al., 2015, V. Krashevska unpubl. data). Monte-Carlo tests (999 permutations) were performed to evaluate the overall model significance and the significance of environmental variables and individual axes. Since the global test with all environmental variables was significant, we used forward selection to identify the most important environmental variables affecting Collembola communities. The forward selection procedure was stopped if a variable reached a level of significance $>0.05$.

\section{Results}

Variations in density of total Collembola with land-use system, season, layer and life form

In total 9543 individuals were assigned to 54 species from 27 genera and 13 families across all land-use systems and four sampling dates. Collembola density varied between landuse systems, layers and season with the three factor interaction being significant $(p=0.0032$, Table 5.2, Appendix 5.5). On average across seasons, the density of Collembola was highest in rainforest and lowest in oil palm plantations both in litter and soil layer. Collembola density in all land-use system in the litter layer was highest at the early wet season (November). In the litter layer, Collembola density in rainforest was lowest in the dry season (June), then increased and reached the highest density at the beginning of wet season (November), whereas Collembola density in oil palm plantations was highest at the beginning of the dry season (June) (Fig.5.1.A). In the soil layer, the density of Collembola in all land-use systems was highest at the end of the dry season (August) and decreased during wet season (Fig.5.1.B). 


\section{Chapter 5}

Table 5.2. Results of statistical analyses: total Collembola, epedaphic, hemiedaphic, euedaphic life forms separately

\begin{tabular}{|c|c|c|c|}
\hline Factor & D.f. & F-value & p-value \\
\hline \multicolumn{4}{|l|}{ TOTAL } \\
\hline Land-use system & 2 & 48.20 & $<0.001 * * *$ \\
\hline Layer & 1 & 0.06 & 0.79 \\
\hline Sampling date & 3 & 9.50 & $0.023 * * *$ \\
\hline Layer $\times$ System & 2 & 16.10 & $<0.001 * * *$ \\
\hline Layer $\times$ Sampling date & 3 & 11.58 & $0.008 * *$ \\
\hline System $\times$ Sampling date & 6 & 19.96 & $0.002 * *$ \\
\hline Layer $\times$ System $\times$ Sampling date & 6 & 19.61 & $0.002 * *$ \\
\hline \multicolumn{4}{|l|}{ ATMOBIOTIC } \\
\hline Land-use system & 2 & 45.92 & $<0.001 * * *$ \\
\hline Layer & 1 & 0.07 & 0.78 \\
\hline Sampling date & 3 & 17.38 & $<0.001 * * *$ \\
\hline Layer×System & 2 & 45.81 & $<0.001 * * *$ \\
\hline Layer $\times$ Sampling date & 3 & 11.58 & 0.16 \\
\hline System $\times$ Sampling date & 6 & 7.09 & 0.32 \\
\hline Layer $\times$ System $\times$ Sampling date & 6 & 8.20 & 0.22 \\
\hline \multicolumn{4}{|l|}{ EPEDAPHIC } \\
\hline Land-use system & 2 & 5.19 & 0.07 \\
\hline Layer & 1 & 17.76 & $<0.001 * * *$ \\
\hline Sampling date & 3 & 6.44 & 0.09 \\
\hline Layer $\times$ System & 2 & 0.09 & 0.95 \\
\hline Layer $\times$ Sampling date & 3 & 21.92 & $<0.001 * * *$ \\
\hline System $\times$ Sampling date & 6 & 18.42 & $0.005 * *$ \\
\hline Layer $\times$ System $\times$ Sampling date & 6 & 12.86 & 0.04 \\
\hline \multicolumn{4}{|l|}{ EUEDAPHIC } \\
\hline Land-use system & 2 & 23.41 & $<0.001 * * *$ \\
\hline Layer & 1 & 2.09 & 0.14 \\
\hline Sampling date & 3 & 0.31 & 0.95 \\
\hline \multicolumn{4}{|l|}{ HEMIEDAPHIC } \\
\hline Land-use system & 2 & 3.23 & 0.19 \\
\hline Layer & 1 & 7.63 & $0.005 * *$ \\
\hline Layer×System & 2 & 1.51 & 0.46 \\
\hline
\end{tabular}




\section{Chapter 5}

A 45000

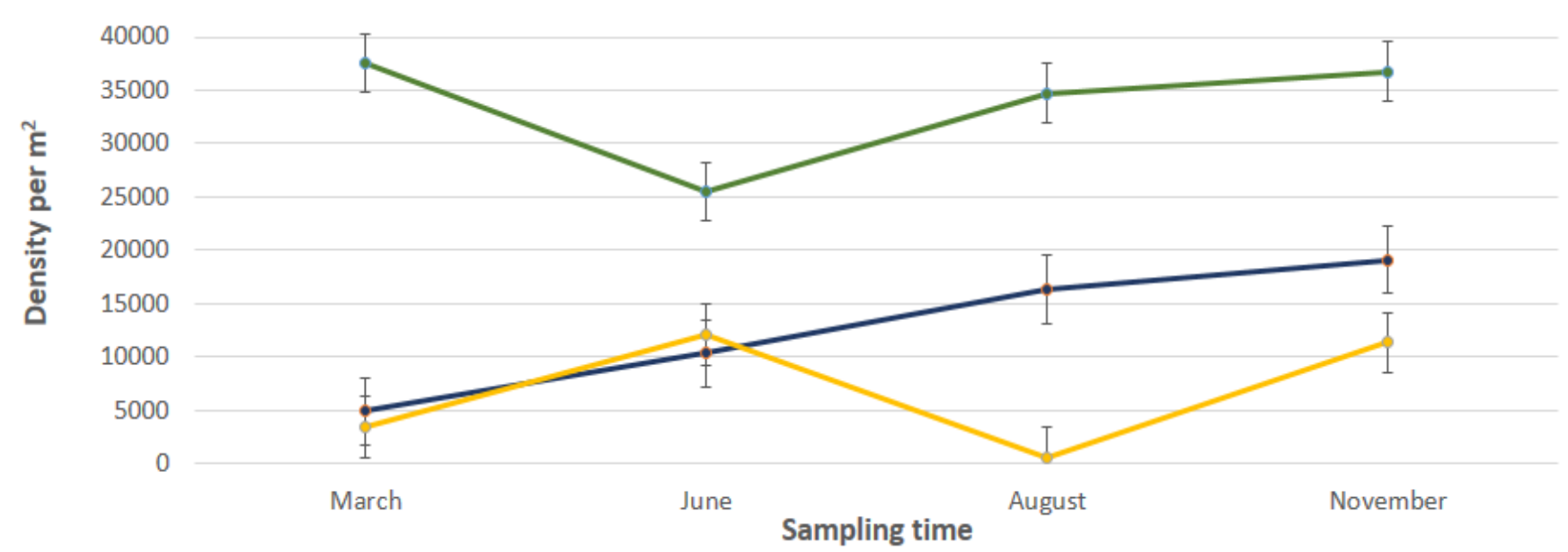

B

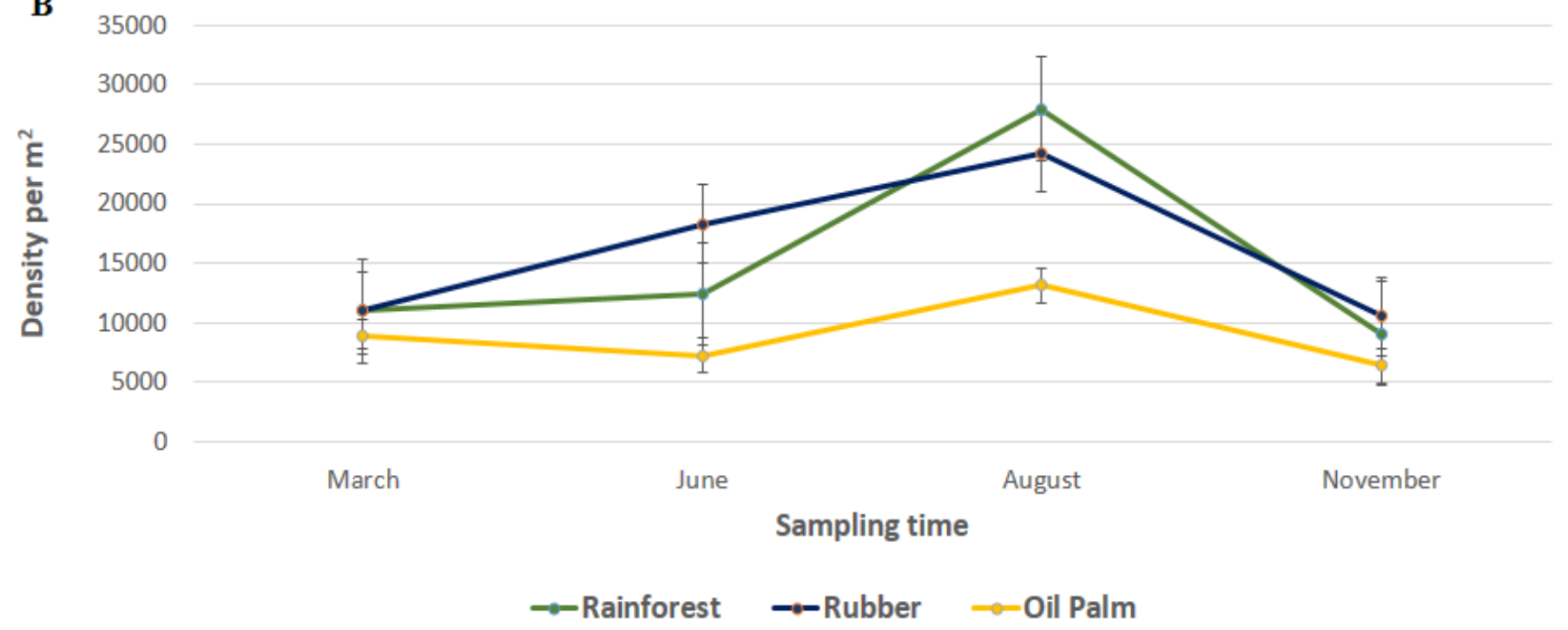

Figure 5.1. Density of Collembola in litter and soil layer in different sampling times (March, June, August, November) from different land-use systems. 


\section{Chapter 5}

\section{Variations in density of Collembola life forms with land-use system, layer and season}

Concerning to the vertical stratification, seasonal variation did not affect density of euedaphic and hemiedaphic species. On the other hand, atmobiotic and epedaphic species were affected by system, season and layer. Results of statistical analysis are available in Table 5.2 with the model of all life forms are available in Appendix 5.6.

\section{Seasonal variations in community composition of Collembola in the litter and soil layer}

Of the 54 species identified, $54.2 \%$ were present in each of the land-use systems, $8.3 \%$ were only found in rainforest, $2.1 \%$ only in rubber plantations, and $6.3 \%$ only in oil palm plantations. Folsomides centralis, Isotomiella spp. and Pseudosinella sp. were most abundant across land-use systems. Manova results from NMDS axes showed that Collembola community composition were affected by systems ( $\mathrm{p}$ value $=<0.001$ ), sampling dates ( $\mathrm{p}$ value $=0.006$ ), and interaction between systems and sampling dates $(\mathrm{p}$ value $=<0.013)$ and also interaction between systems and layer $(\mathrm{p}$ value $=0.001)$, for detail see Table 5.3.

Table 5.3. Manova test of interaction between season, system and layer from NMDS of Collembola community composition

\begin{tabular}{lllll}
\hline & Df & Pillais trace & Approx. F & $\operatorname{Pr}(>\mathrm{F})$ \\
\hline Season & 3 & 0.43007 & 2.2425 & $0.006263 * *$ \\
System & 3 & 0.76237 & 4.5655 & $1.852 \mathrm{e}-07 * * *$ \\
Layer & 1 & 0.05775 & 0.7968 & 0.555966 \\
Season $\times$ System & 9 & 0.85358 & 1.5782 & $0.013530 *$ \\
Season $\times$ Layer & 3 & 0.30679 & 1.5264 & 0.098379. \\
System $\times$ Layer & 1 & 0.25510 & 4.4521 & $0.001504 * *$ \\
Season $\times$ System $\times$ Layer & 3 & 0.26419 & 1.2940 & 0.208367 \\
\hline
\end{tabular}

Based on manova, the interaction between seasonal variation and system had a pronounced effect on the species composition of Collembola communities. Continuing with LDA, Collembola communities composition in all land-use systems differed at every sampling date with the first LDA axis explaining 98\%, 83\% 96\% and 74\% of the variation in March, June, August and November, respectively (Table 5.4; Fig. 5.2A-D). Species composition of Collembola communities in the litter layer was different at each sampling date (March, June, August, November) across the land-use systems with separation of communities being more pronounced in the dry season (June) than at the beginning of the wet season (November). 
Chapter 5
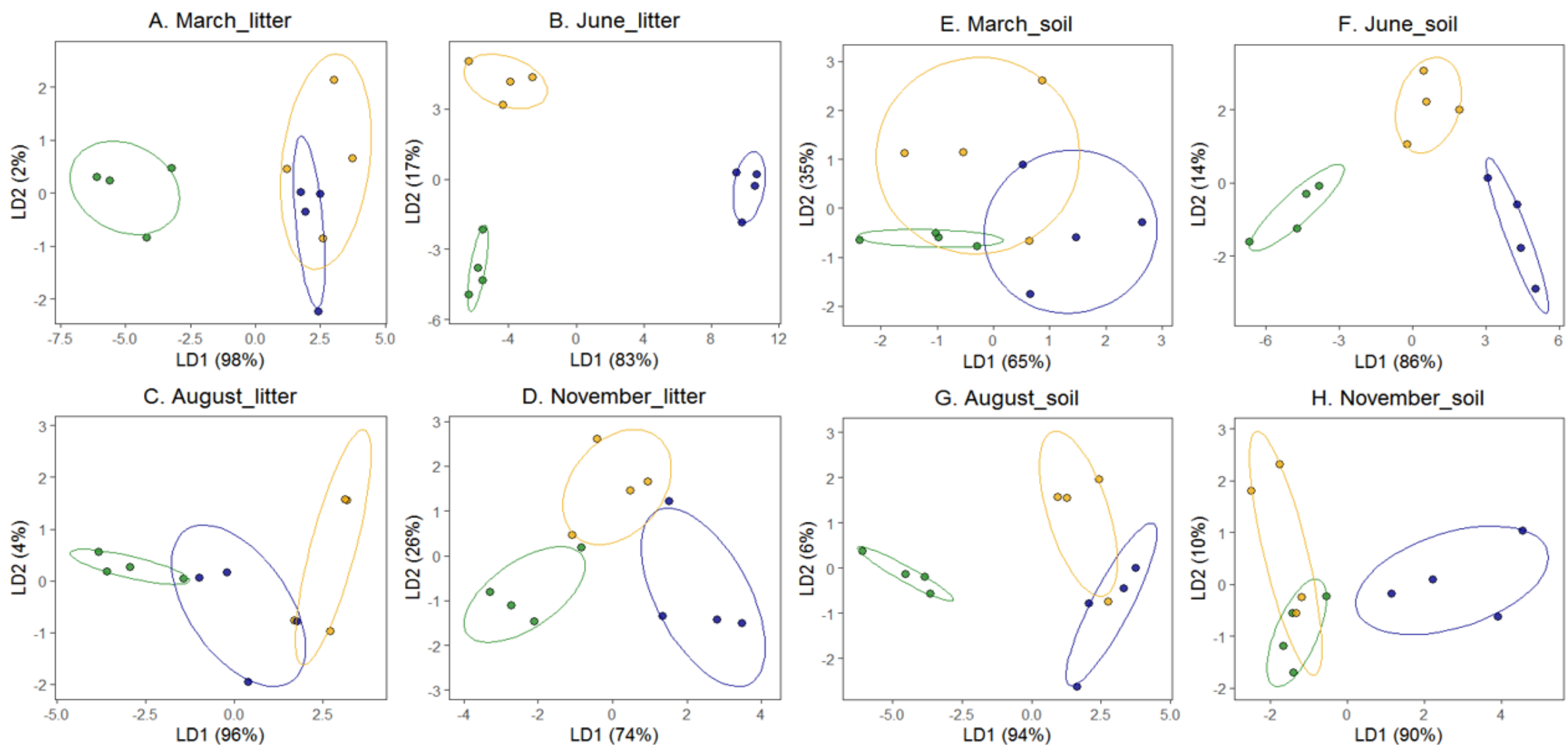

System - Rainforest - Rubber - Oil palm

Figure 5.2. Linear Discriminant Analysis (LDA) of species community composition of litter and soil Collembola in different seasons and land-use systems). Land-use system was used as grouping variable (Rainforest=forestgreen, Rubber=darkblue, Oil palm= darkgoldenrot). Ellipses were calculated using the MASS package in $R$ to visualize the grouping of species or traits in the different land-use systems. Each point represents a sample. 


\section{Chapter 5}

Season also significantly affected the species composition of Collembola communities in the soil layer. The first LDA axis of March explaining 65\% of the variation (Table 5.4; Fig. 5.2E), the first LDA axis of June explaining $86 \%$ of the variation (Table 5.4; Fig. 5.2F), the first LDA axis of August explaining 94\% (Table 5.4; Fig. 5.2G), and the first LDA axis of November explaining 90\% (Table 5.4; Fig. 5.2H). In line with the communities in the litter layer, species composition of Collembola communities in the soil layer also differed at each sampling date (March, June, August, November) across the land-use systems which the separation being most pronounced in the dry season (June) and less at the end of wet season (March).

Table 5.4. Wilks' lambda and p-value of LDA from every sampling date and layer

\begin{tabular}{lllc}
\hline Sampling date & Layer & Wilks' lambda & approx. F \\
\hline March & Litter & 0.045 & $14.74^{* * *}$ \\
June & Litter & 0.001 & $127.10^{* * *}$ \\
August & Litter & 0.09 & $8.78^{* * *}$ \\
November & Litter & 0.07 & $11.19^{* * *}$ \\
March & Soil & 0.24 & $4.18^{* *}$ \\
June & Soil & 0.02 & $31.38^{* * *}$ \\
August & Soil & 0.03 & $17.47^{* * *}$ \\
November & Soil & 0.09 & $9.61^{* * *}$ \\
\hline
\end{tabular}

Chi-square values; ${ }^{*} p<0.05 ;^{* *} p<0.01 ; * * *<0.001$

\section{Environmental factors related to Collembola community composition}

In the litter layer, four of the nine environmental variables significantly correlated with species composition (CCA, forward selection) explaining $16.7 \%$ of the variation in species composition $($ Trace $=0.57, \mathrm{~F}=1.23, p=0.004$; Fig. 5.3A). Water content accounted for $4.9 \%$ (pseudo $\mathrm{F}=2.2, p=0.006)$, the fungal PLFA marker $(18: 2 \omega 6,9)$ for $4.3 \%$ (pseudo $\mathrm{F}=2.0, p=$ 0.017 ), the mycorrhizal marker $(16: 1 \omega 5)$ for $3.8 \%$ (pseudo $\mathrm{F}=1.8, p=0.030$ ) and litter $\mathrm{pH}$ for 3.7 $\%$ (pseudo $\mathrm{F}=1.8, p=0.039$ ) of the total variation in species composition. The first axis separated rainforest from rubber and oil palm plantations at all sampling dates, whereas the second axis separated sampling dates in the dry season (June and August) from those in the wet season (November and March) across land-use systems (with the exception of November in oil palm plantations). Community composition in rainforest in the wet season (November and March) correlated closely with litter water content, whereas community composition in plantation systems generally correlated with litter $\mathrm{pH}$, but during the wet season also with fungal and AMF marker PLFAs, especially in rubber plantations. Some euedaphic species (i.e., Isotomiella $c f$. minor, 
Isotomiella symetrimucronata, Folsomina onychiurina, Thalassaphorura, Megalothorax cf. minor) were associated with higher water content in rainforest in the wet season. A number of atmobiotic and epedaphic species, (i.e., Dicranocentrus sp.1, Ptenothrix sp.1, Homidia cingula, Isotomurus cf. parabalteatus, Folsomides centralis) were associated with low water content and high $\mathrm{pH}$ in plantations in the dry season. Interestingly, fungi only played a role in plantation systems in the wet season. 
A

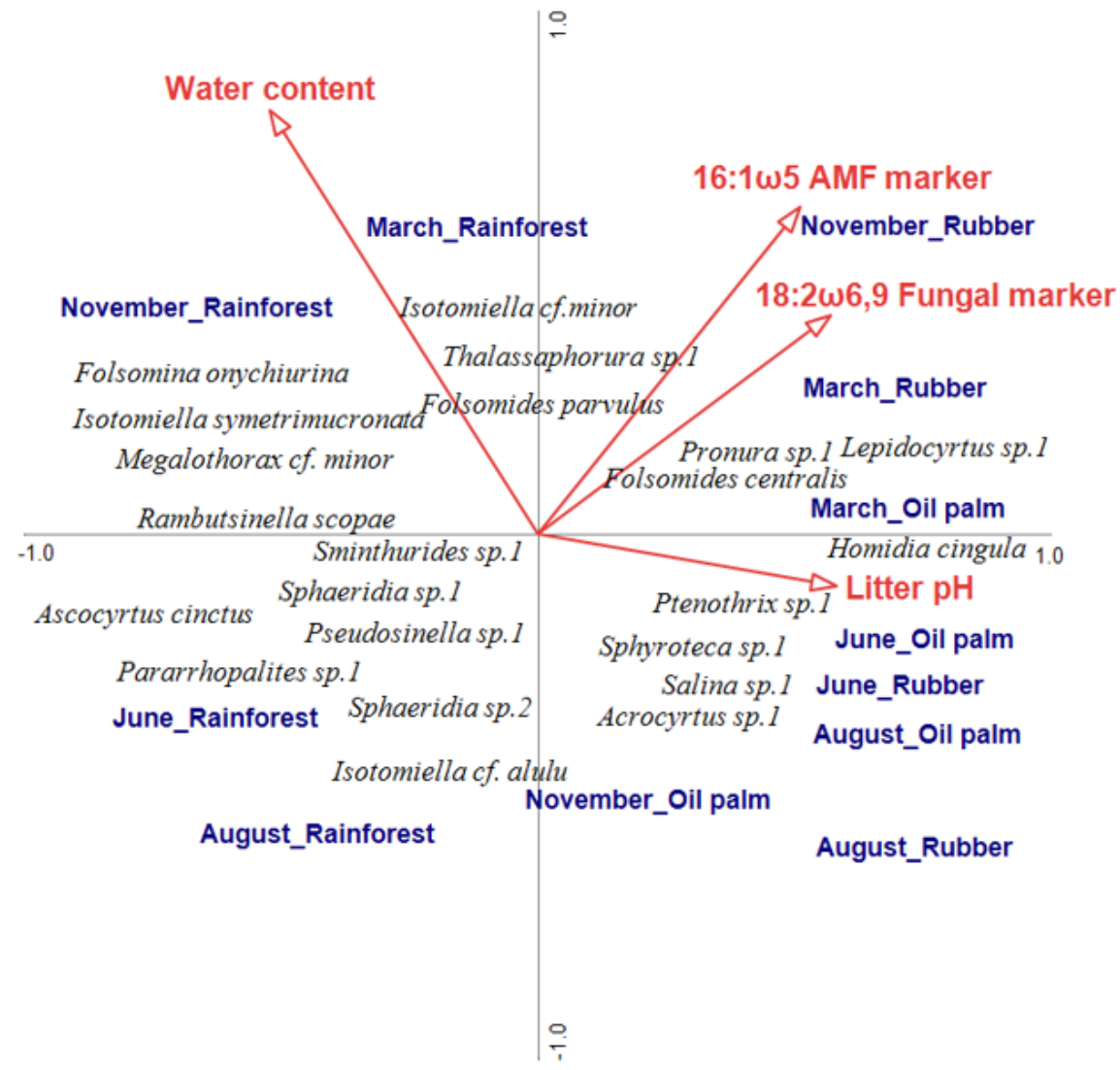

B

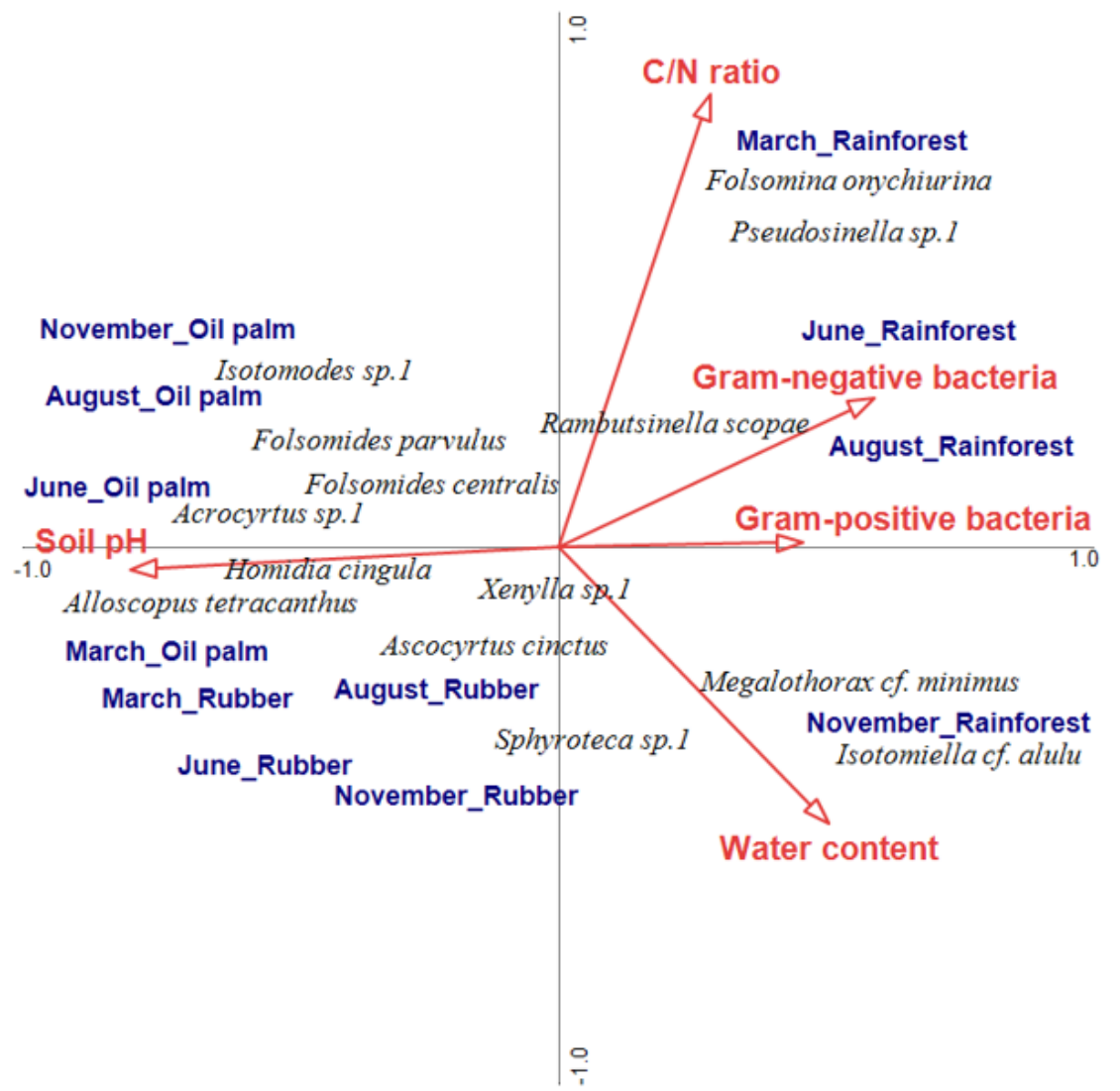

Figure 5.3. Canonical correspondence analysis of collembolan species in litter and soil layer as related to environmental variables (red arrows). Only environmental variables selected as being significant in the forward selection procedure are shown. The three land-use systems (rainforest, rubber plantations, oil palm plantations) and four seasons (March, June, August, November) were included as silent variables not affecting the ordination. The name of species and land-use systems were centred to their position. The length of arrows represent the percentage of variation explained by environmental variables. 
Overall, environmental factors in soil explained a total of $25.7 \%$ of the variation in community composition thereby exceeding that in litter (Trace $=0.49, \mathrm{~F}=1.77, p=0.002$; Fig. 5.3B). Five of the nine environmental variables significantly correlated with species composition, with soil $\mathrm{pH}$ accounting for $6.6 \%$ (pseudo $\mathrm{F}=3.5, p=0.002$ ), water content for $5.5 \%$ (pseudo $\mathrm{F}$ $=2.6, p=0.009$ ), $\mathrm{C} / \mathrm{N}$ ratio for $4.9 \%$ (pseudo $\mathrm{F}=2.4, p=0.012$ ), Gram-positive bacteria marker for $4.7 \%$ (pseudo $\mathrm{F}=2.1, p=0.021$ ), and Gram-negative bacteria marker for $4.0 \%$ (pseudo $\mathrm{F}=$ 2.1, $p=0.024)$. Similar to the litter layer, the first axis separated rainforest from rubber and oil palm plantations across sampling dates, whereas the second axes separed the sampling dates within each of the land-use systems with the separation in rubber being less pronounced. Soil $\mathrm{pH}$ was associated with the beginning of the dry season (March and June) particularly in rubber and oil palm plantations. In line with the litter layer, water content correlated with communities at the beginning of the wet season (November) in the soil layer of rainforest with high density of euedaphic species (Isotomiella cf. alulu and Megalothorax cf. minimus). Further, C/N ratio also correlated with the wet season in rainforest (March) with high density of euedaphic species (Pseudosinella sp.1, Folsomina onychiurina). Gram-negative and Gram-positive bacterial markers were associated with the dry season (June and August) in rainforest and with high density of Rambutsinella scopae.

\section{Discussion}

Seasonal variation affected density and community composition of Collembola in rainforest and plantation systems, with the effect being more pronounced in litter than in soil, and for epedaphic and hemiedaphic than for euedaphic species. Seasonal fluctuations were generally similar acrooss land-use systems; the density of Collembola increased in the wet season in the litter layer but decreased in the soil layer. Water content, $\mathrm{pH}$ and $\mathrm{C} / \mathrm{N}$ ratio were identified as important abiotic factors driving Collembola community composition across seasons, while microbial community composition (Gram-positive and Gram-negative bacteria, total and arbuscular mycorrhizal fungi) was identified as important biotic driving factor in particular in the wet season.

\section{Variation in Collembola density between land-use systems and seasons}

Partly confirming our first hypothesis, the density of Collembola changed strongly with rainforest conversion to agricultural plantations along different sampling dates with opposite 


\section{Chapter 5}

patterns in litter and soil. In the litter layer, the density increased in the wet season, particularly early in the wet season, whereas in the soil layer density of Collembola strongly decreased. This is consistent to results of the study of Levings and Windsor (1984) showing that higher litter moisture was associated with higher Collembola density. In the soil layer, Collembola density was at the maximum at the end of the dry season, suggesting that moisture conditions in soil allowed Collembola to thrive throughout the dry season. The results are in line with those of the study of Frith and Frith (1990) in Australian tropical upland rainforests, where litter invertebrate density increased with increasing precipitation, whereas it declined when the litter became dry. Similarly, Muturi et al. (2009) reported Collembola density to decline in the dry season at various sites in tropical Kenya. Generally, decreasing density of Collembola in the dry season has been reported in a number of studies (Smit and Van Gestel, 1997; Detsis, 2000; Bandyopadhyaya et al., 2002; Wiwatwitaya and Takeda, 2005; Mayvan et al., 2015). In the litter layer, differences in Collembola density in rainforest and plantation systems were more pronounced in the dry than in the wet season suggesting that soil moisture is an important factor driving Collembola density with detrimental effects of low moisture being more pronounced in plantations. Furthermore, in the litter layer of rubber plantations, the density of Collembola increased gradually in the rainy season confirming the importance of litter moisture as driving factor for Collembola density. This indicates that humidity is among the most important factors driving the survival, reproduction and vertical stratification of Collembola in tropical forest soils. This fits to the CCA results which confirmed water content as important factor driving Collembola community composition both in litter and soil.

Supporting our second hypothesis, the composition of Collembola life forms varied with season with euedaphic species varying less than epedaphic and hemiedaphic species across landuse systems, presumably reflecting the buffering ability of soil against environmental changes. Euedaphic Collembola are able to withstand even extreme weather conditions (Bokhorst et al., 2012) and have been shown to respond little to changes in abiotic factors associated with changes in forest management (Chauvat et al., 2003). However, in agricultural systems of the temperate zone euedaphic Collembola also sensitively responded to seasonal changes in climate and crop rotation (Kanal, 2004). The study of Makkonen et al. (2011) reported certain Collembola species to be well adapted to drought. Overall, the density and thus the contribution of euedaphic Collembola to ecosystem functioning of tropical soils is more stable in time than that of upper litter-dwelling species. 


\section{Variation in Collembola community composition between land-use systems and seasons}

Unexpectedly, based on the LDA results, the community composition of Collembola at every sampling date differed to a similar extent between rainforest and monoculture plantations. Community composition of Collembola changed strongly with rainforest conversion into agricultural plantations and seasonal variation with the changes being more pronounced in litter than in soil. The more pronounced response in litter presumably reflects the more pronounced exposure to disturbances of Collembola in litter than in soil. Overall, the differences in Collembola community composition were more pronounced during the dry than during the wet season, presumably due to adaptation of species to environmental changes during drought. In the litter layer, the community composition of Collembola in rainforest was separated from that in rubber and oil palm plantations, except at the third sampling date (August). This indicates that at the end of the dry season, Collembola community composition becomes more similar across land-use systems. Interestingly, confirming the LDA results, the distinction of community composition were very clear in the dry season both in litter and soil layer, presumably this indicates that species in different habitat types have different adaptation modes to climate changes. Based on the previous study at the same sites, the Collembola community in the study area is dominated by species of the family Isotomidae, particularly the genera Folsomides and Isotomiella and the family Entomobryidae, particularly genera Pseudosinella and Ascocyrtus (Susanti et al., 2021) reflecting that these species are well adapted to environmental conditions in agro-ecosystems.

\section{Environmental factors driving Collembola community composition between land-use systems and seasons}

As indicated by CCA, water content and $\mathrm{pH}$ were the most important environmental factors driving the structure of Collembola communities in both litter and soil across seasons. The relevance of water content for Collembola community composition has been stressed repeatedly in studies from the temperate zone (Ponge, 1983; Fujii et al., 2014). Both in the litter and soil layer, $\mathrm{pH}$ correlated with the community composition of Collembola in the dry season of plantation systems and water content with community composition in rainforest during the wet season. This indicates that water content functions as important environmental factor in rainforest, especially during the wet season. High amounts of organic matter and high water holding capacity in rainforest likely improves habitat conditions and increases the availability of food resources in 


\section{Chapter 5}

particular for eudaphic Collembola (Muturi et al., 2011) correlating with high density of euedaphic species. The number of euedaphic species, including Pseudosinella, Megalothorax cf. minimus and Isotomiella were associated with high water content in rainforest. Further, $\mathrm{pH}$ is driving communities of Collembola in plantations systems, especially during the dry season. The strong effect of $\mathrm{pH}$ in structuring species composition of Collembola likely is related to the increase in soil $\mathrm{pH}$ with the conversion of rainforest into plantations. Species in rubber and oil palm plantations, such as Homidia cingula, Isotomurus cf. parabalteatus, Dicranocentrus sp.1 and Acrocyrtus sp.1., were associated with high $\mathrm{pH}$ and reached high abundance in the dry season.

Beside abiotic environmental factors, PLFA markers for fungi and AMF correlated with Collembola community composition in the litter layer of plantation systems during the wet season. The is in line with the response of arbuscular mycorrhizal fungi benefitting from high $\mathrm{pH}$ (Wang et al., 1993; Van Aarle et al., 2002; Carrino-Kyker et al., 2016). A'Bear et al. (2012) also reported temperature to influence fungal mycelia. Furthermore, in the soil layer of rainforest in the dry season (June and August) Collembola community correlated positively with Gram-positive and Gram-negative bacteria. This is in line with the study of Chauvat et al. (2003), which reported that in forest ecosystems Collembola positively correlated with the abundance of bacteria. In addition to directly affecting Collembola by increasing the risk of desiccation, reduced soil moisture in the dry season may also reduce the availability of microorganisms as food (Fierer et al., 2003; Rousk and Bååth, 2007; Rousk et al., 2010b; Zhang et al., 2013). In the dry season, soil temperature in plantation systems with less litter in the litter layer was higher than in rainforest, especially in oil palm plantations, and this may explain the low density of Collembola in the dry season.

Further, the results from CCA indicate that in the wet season, food availability and quality, represented by higher litter $\mathrm{C} / \mathrm{N}$ ratio in rainforest and higher fungal PLFA marker in plantations, are important factors driving Collembola community composition. This is in line with the study by Cassagne et al. (2003) who reported that litter $\mathrm{C} / \mathrm{N}$ ratio is an important factor driving Collembola community composition in particular at high humidity, fitting well to studies from tropical regions reporting that litter $\mathrm{C} / \mathrm{N}$ ratio is higher in rainforest than in plantation systems, especially in the wet season (Cavelier and Tobler, 2000; Guillaume et al., 2016; Violita et al., 2016). Interestingly, in the soil layer, $\mathrm{pH}$ correlated negatively with litter $\mathrm{C} / \mathrm{N}$ ratio, reflecting that higher $\mathrm{pH}$ is more important in the dry season in plantations, whereas litter $\mathrm{C} / \mathrm{N}$ ratio is more important in the wet season in rainforest. 


\section{Chapter 5}

Overall, CCA results suggested that in both litter and soil euedaphic species were most responsive in the wet season in particular in rainforest, whereas epedaphic and atmobiotic species were most responsive in the dry season in particular in plantation systems. Pseudosinella sp.1, Isotomiella cf. minor were the dominant species in each of the land-use systems studied and these species are categorized as fungivores, which is in line with the results from stable isotopic results (Susanti et al., 2021) and generally appear to dominante in tropical forests (Takeda 1996). This is further supported by results of the CCA in which Collembola species in plantation systems correlated closely with PLFA markers of total and mycorrhizal fungi. Fungi only correlated with Collembola community composition in plantations system in the wet season suggesting that the role of resources is more pronounced when climatic condition are beneficial.

\section{Conclusion}

Collembola density in the different land-use systems studied varied significantly with season. Overall, Collembola density in the litter layer increased in the wet season (November, March), whereas in soil the density strongly decreased in the early wet season irrespective of the land-use system. Collembola community composition in litter and soil differed significantly with the differences being most pronounced during the dry season. Water content and $\mathrm{pH}$ were identified as the most important factors driving Collembola community composition both in litter and soil across seasons with water content correlating with Collembola community composition in rainforest in the wet season and $\mathrm{pH}$ in plantation systems in the dry season.

\section{Acknowledgements}

This study was funded by the Deutsche Forschungsgemeinschaft (DFG, German Research Foundation) - project number 192626868 - SFB 990 in the framework of the collaborative German - Indonesian research project CRC990. We thank the State Ministry of Research and Technology of Indonesia (RISTEK), the Indonesian Institute of Sciences (LIPI), Ministry of Forestry (PHKA) and Restoration Ecosystem Indonesia Harapan for the permits. Sampling of animals used in this study was based on Collection permit No. 2841/IPH.1/KS.02.04/X/2016 issued by the Indonesian Ministry of Forestry (PHKA). We thank Indonesian organizations and farmers for granting access to the sampling plots and use of their properties. Special gratitude goes to Feng Zhang, Louis Deharveng and Anne Bedos for verification of Collembola morphospecies identification. We also thank Penelope Greenslade for sharing her unpublished drafts of the key on Indonesian Collembola. 


\section{Appendix}

Appendix 5.1. Environmental factors in litter layer per land-use system and season (S1-March, S2-June, S3-August, S4-November)

\begin{tabular}{|c|c|c|c|c|c|c|c|c|c|}
\hline System & $\begin{array}{l}\text { Microbial } \\
\text { Carbon }\end{array}$ & $\begin{array}{l}\text { Litter } \\
\text { pH }\end{array}$ & $\begin{array}{l}\text { C/N } \\
\text { Ratio }\end{array}$ & $\begin{array}{l}16: 1 \omega 5 \\
\text { AMF } \\
\text { marker }\end{array}$ & $\begin{array}{l}18: 2 \omega 6.9 \\
\text { Fungal } \\
\text { marker }\end{array}$ & $\begin{array}{l}20: 5 \omega 3 \\
\text { Algae } \\
\text { marker }\end{array}$ & $\begin{array}{l}\text { Gram(-) } \\
\text { Bacteria }\end{array}$ & $\begin{array}{l}\text { Gram(+) } \\
\text { Bacteria }\end{array}$ & Water content \% \\
\hline S1_Rainforest & 10933.8 & 4.52 & 23.6 & 6.35 & 49.80 & 0.13 & 30.17 & 45.38 & 81.8 \\
\hline S1_Rainforest & 5741.0 & 4.02 & 28.3 & 4.78 & 73.04 & 0 & 34.57 & 38.61 & 144.21 \\
\hline S1_Rainforest & 5809.2 & 3.53 & 24.4 & 6.93 & 66.91 & 0.21 & 44.50 & 59.19 & 154.14 \\
\hline S1_Rainforest & 6176.0 & 3.61 & 31.7 & 5.54 & 77.31 & 0.03 & 38.76 & 50.24 & 97 \\
\hline S1_Oil palm & 26171 & 5.22 & 27.0 & 8.49 & 59.56 & 0.33 & 52.11 & 84.09 & 59.96 \\
\hline S1_Oil palm & 8614.2 & 5.25 & 28.9 & 6.12 & 51.01 & 0.01 & 34.72 & 53.64 & 13.87 \\
\hline S1_Oil palm & 5146.8 & 4.25 & 25.7 & 2.98 & 89.46 & 0.01 & 21.07 & 34.94 & 26.39 \\
\hline S1_Oil palm & 8951.9 & 5.93 & 25.2 & 6.71 & 30.12 & 0.79 & 60.19 & 102.56 & 7.95 \\
\hline S1_Rubber & 16437.1 & 5.08 & 28.6 & 6.14 & 105.65 & 0.12 & 36.73 & 58.98 & 37.52 \\
\hline S1_Rubber & 22668.1 & 4.98 & 23.4 & 8.88 & 25.80 & 0.07 & 35.52 & 56.48 & 33.31 \\
\hline S1_Rubber & 13800.6 & 4.97 & 21.6 & 9.03 & 67.93 & 0.01 & 33.91 & 46.77 & 44.93 \\
\hline S1_Rubber & 18053.6 & 4.78 & 28.5 & 6.72 & 89.53 & 0.01 & 42.57 & 55.80 & 54.83 \\
\hline S2_Rainforest & 6931.7 & 3.96 & 33.2 & 4.44 & 60.62 & 0 & 45.51 & 37.96 & 17.91 \\
\hline S2_Rainforest & & 3.92 & 25.7 & 4.79 & 37.67 & 0 & 44.61 & 43.61 & 151.55 \\
\hline S2_Rainforest & 14613.3 & 3.96 & 25.6 & 6.65 & 51.52 & 0 & 48.76 & 53.86 & 65.57 \\
\hline S2_Oil palm & 6159.5 & 5.3 & 26.2 & 4.57 & 52.07 & 0 & 30.80 & 36.32 & 55.58 \\
\hline S2_Oil palm & 4138.6 & 4.84 & 32.7 & 4.57 & 41.91 & 0 & 27.55 & 34.23 & 22.4 \\
\hline S2_Oil palm & 5595.9 & 4.62 & 24.7 & 3.07 & 123.72 & 0 & 67.28 & 35.63 & 39.15 \\
\hline S2_Oil palm & 5600.2 & 6.18 & 36.8 & 6.96 & 93.47 & 0 & 90.21 & 72.89 & 22.92 \\
\hline S2_Rubber & 8598.9 & 5.27 & 28.4 & 6.35 & 101.65 & 0 & 65.54 & 45.84 & 20.99 \\
\hline S2_Rubber & 13656.4 & 5.17 & 27.2 & 4.25 & 57.39 & 0 & 46.52 & 46.72 & 35.27 \\
\hline S2_Rubber & 7568.0 & 4.97 & 32.4 & 2.74 & 101.19 & 0 & 39.99 & 27.39 & 38.09 \\
\hline S2_Rubber & 6007.0 & 4.5 & 30.6 & 2.88 & 109.04 & 0 & 44.57 & 33.10 & 41.28 \\
\hline S3_Rainforest & 6742.1 & 4.47 & 27.6 & 2.13 & 32.46 & 0 & 30.29 & 21.14 & 64.98 \\
\hline S3_Rainforest & 5578.3 & 4.11 & 30.3 & 2.25 & 22.06 & 0 & 24.10 & 27.51 & 68.27 \\
\hline
\end{tabular}


Chapter 5

\begin{tabular}{|c|c|c|c|c|c|c|c|c|c|}
\hline S3_Rainforest & 5644.5 & 4.26 & 27.2 & 3.50 & 30.64 & 0 & 32.66 & 30.89 & 59.22 \\
\hline S3_Oil palm & 6264.3 & 3.63 & 31.8 & 3.18 & 23.74 & 0 & 29.57 & 31.95 & 72.82 \\
\hline S3_Oil palm & 6806.5 & 5.29 & 35.5 & 2.83 & 88.67 & 0 & 18.65 & 26.07 & 55.9 \\
\hline S3_Oil palm & 9068.9 & 6.52 & 38.1 & 6.01 & 62.39 & 0 & 55.53 & 61.69 & 25.33 \\
\hline S3_Oil palm & 3377.8 & 4.35 & 24.5 & 5.27 & 123.79 & 0 & 21.55 & 29.32 & 25.65 \\
\hline S3_Rubber & 4896.3 & 4.76 & 41.6 & 3.14 & 75.89 & 0 & 17.58 & 25.29 & 74.69 \\
\hline S3_Rubber & 8320.4 & 5.65 & 35.1 & 2.27 & 45.16 & 0 & 29.48 & 17.50 & 88.61 \\
\hline S3_Rubber & 18763.4 & 5.8 & 28.9 & 3.87 & 0 & 0 & 30.97 & 20.08 & 77.42 \\
\hline S3_Rubber & 7019.1 & 5.6 & 35.1 & 6.25 & 0 & 1.02 & 79.27 & 64.59 & 88.36 \\
\hline S4_Rainforest & 4200.8 & 4.42 & 31.4 & 4.13 & 104.68 & 0 & 112.19 & 63.34 & 50.19 \\
\hline S4_Rainforest & 7296.1 & 4.61 & 26.1 & 2.61 & 37.42 & 0 & 48.25 & 38.45 & 64.95 \\
\hline S4_Rainforest & 5176.3 & 4.21 & 30.1 & 1.58 & 40.10 & 0 & 27.18 & 17.88 & 200.7 \\
\hline S4_Rainforest & 11062.7 & 4.26 & 31.1 & 2.22 & 20.85 & 0 & 58.53 & 24.29 & 122.02 \\
\hline S4_Oil palm & 14587.5 & 3.99 & 31.9 & 1.97 & 24.42 & 0 & 86.87 & 13.23 & 68.49 \\
\hline S4_Oil palm & 25157.7 & 5.63 & 24.3 & 3.30 & 11.46 & 0 & 37.61 & 75.01 & 66.25 \\
\hline S4_Oil palm & 16624.2 & 5.34 & 28.3 & 3.07 & 26.56 & 0 & 33.68 & 49.29 & 71.81 \\
\hline S4_Oil palm & 3947.1 & 4.94 & 21.4 & 2.61 & 44.25 & 0 & 49.94 & 52.19 & 124.54 \\
\hline S4_Rubber & 10480.4 & 5.74 & 26.5 & 4.94 & 98.94 & 0 & 87.86 & 131.69 & 91.27 \\
\hline S4_Rubber & 13253.1 & 5.78 & 22.8 & 7.83 & 114.70 & 0 & 71.61 & 95.45 & 109.29 \\
\hline S4_Rubber & 7141.5 & 5.51 & 22.8 & 11.28 & 48.27 & 0 & 73.41 & 132.21 & 62.15 \\
\hline S4_Rubber & 15062.9 & 5.03 & 23.6 & 9.94 & 74.91 & 0 & 132.92 & 143.57 & 115.26 \\
\hline
\end{tabular}


Appendix 5.2. Environmental factors in soil layer per land-use system (rainforest, rubber, oil palm) and season (April, June, August, November).

\begin{tabular}{|c|c|c|c|c|c|c|c|c|c|}
\hline System & $\begin{array}{l}\text { Microbial } \\
\text { C }\end{array}$ & $\begin{array}{l}\text { Soil } \\
\mathrm{pH}\end{array}$ & $\mathrm{C} / \mathrm{N}$ ratio & $\begin{array}{l}16: 1 \omega 5 \\
\text { AMF } \\
\text { marker }\end{array}$ & $\begin{array}{l}18: 2 \omega 6.9 \\
\text { Fungal } \\
\text { marker }\end{array}$ & $\begin{array}{l}20: 5 \omega 3 \\
\text { Algae } \\
\text { marker }\end{array}$ & $\begin{array}{l}\operatorname{Gram}(-) \\
\text { bacteria }\end{array}$ & $\begin{array}{l}\operatorname{Gram}(+) \\
\text { bacteria }\end{array}$ & $\begin{array}{l}\text { Water } \\
\text { content } \%\end{array}$ \\
\hline S1_Rainforest & 570.5 & 4 & 14.8 & 1.57 & 1.82 & 0 & 8.15 & 20.53 & 242.33 \\
\hline S1_Rainforest & 477.1 & 3.92 & 13.8 & 1.21 & 0.90 & 0 & 0.91 & 21.21 & 269.24 \\
\hline S1_Rainforest & 475.7 & 3.63 & 14.7 & 2.10 & 1.35 & 0 & 2.21 & 24.06 & 267.36 \\
\hline S1_Rainforest & 618.3 & 3.63 & 14.9 & 2.20 & 1.74 & 0 & 11.59 & 24.96 & 242.9 \\
\hline S1_Oil palm & 836.0 & 5.44 & 13.3 & 4.23 & 2.41 & 0.03 & 14.07 & 32.32 & 248.07 \\
\hline S1_Oil palm & 213.6 & 4.35 & 12.7 & 1.77 & 1.14 & 0.01 & 2.81 & 17.02 & 263.24 \\
\hline S1_Oil palm & 266.0 & 4.37 & 13.3 & 0.40 & 0.18 & 0 & 4.12 & 12.12 & 241.33 \\
\hline S1_Oil palm & 866.5 & 4.85 & 16.8 & 2.11 & 1.00 & 0.01 & 8.39 & 23.88 & 246.97 \\
\hline S1_Rubber & 309.6 & 4.14 & 11.8 & 1.92 & 1.10 & 0 & 3.99 & 17.94 & 243.56 \\
\hline S1_Rubber & 515.7 & 4.16 & 11.5 & 2.10 & 0 & 0 & 4.77 & 21.32 & 247.47 \\
\hline S1_Rubber & 317.4 & 4.24 & 13 & 1.45 & 0.31 & 0 & 1.88 & 17.39 & 235.54 \\
\hline S1_Rubber & 623.5 & 4.12 & 12.9 & 2.91 & 0 & 0 & 6.31 & 31.70 & 241.22 \\
\hline S2_Rainforest & 646.9 & 3.9 & 14.1 & 2.42 & 1.62 & 0 & 11.79 & 24.37 & 245.07 \\
\hline S2_Rainforest & 459.6 & 3.68 & 15.6 & 2.62 & 1.14 & 0 & 19.07 & 40.78 & 247.24 \\
\hline S2_Rainforest & & 3.39 & 17.1 & 3.37 & 1.76 & 0 & 22.84 & 36.37 & 233.16 \\
\hline S2_Rainforest & 602.7 & 3.49 & 15.4 & 3.44 & 2.78 & 0 & 20.69 & 32.57 & 267.49 \\
\hline S2_Oil palm & 502.5 & 4.74 & 12.9 & 2.42 & 1.05 & 0 & 8.19 & 22.47 & 258.77 \\
\hline S2_Oil palm & 246.4 & 4.3 & 11.7 & 1.12 & 0.56 & 0 & 2.71 & 11.57 & 267.21 \\
\hline S2_Oil palm & 181.4 & 4.42 & 12.3 & 2.48 & 0.82 & 0 & 7.25 & 22.75 & 255.21 \\
\hline S2_Oil palm & 426.9 & 4.44 & 14.5 & 1.30 & 0 & 0 & 5.69 & 13.84 & 233.61 \\
\hline S2_Rubber & 348.9 & 4.24 & 11.8 & 2.13 & 1.03 & 0 & 6.18 & 17.51 & 273.88 \\
\hline S2_Rubber & 626.6 & 4.12 & 11.4 & 0 & 0 & 0 & 2.90 & 12.61 & 267.17 \\
\hline S2_Rubber & 246.4 & 4.15 & 13 & 1.58 & 0 & 0 & 4.29 & 15.15 & 250.51 \\
\hline S2_Rubber & 388.1 & 4.05 & 12.6 & 2.82 & 0.63 & 0 & 6.33 & 26.71 & 255.5 \\
\hline S3_Rainforest & 329.9 & 3.76 & 14.9 & 2.60 & 1.15 & 0 & 13.58 & 29.07 & 267.17 \\
\hline S3_Rainforest & 436.1 & 3.72 & 14 & 2.47 & 2.71 & 0 & 12.40 & 26.74 & 272.2 \\
\hline S3_Rainforest & 353.2 & 3.61 & 15.9 & 3.18 & 2.13 & 0 & 18.01 & 33.52 & 247.97 \\
\hline
\end{tabular}


Chapter 5

\begin{tabular}{|c|c|c|c|c|c|c|c|c|c|}
\hline S3_Oil palm & 711.7 & 4.4 & 14.8 & 2.89 & 0.97 & 0 & 10.90 & 29.55 & 251.17 \\
\hline S3_Oil palm & 266.9 & 4.48 & 15.7 & 3.07 & 1.67 & 0 & 10.21 & 25.69 & 234.43 \\
\hline S3_Oil palm & 156.0 & 4.39 & 13.2 & 0 & 0 & 0 & 2.24 & 10.17 & 249.66 \\
\hline S3_Oil palm & 220.5 & 4.15 & 15 & 1.55 & 0 & 0 & 4.84 & 17.62 & 251.95 \\
\hline S3_Rubber & 518.0 & 4.22 & 11.1 & 2.56 & 0.92 & 0 & 12.57 & 24.17 & 243.47 \\
\hline S3_Rubber & 459.0 & 4.13 & 12.1 & 3.89 & 1.25 & 0 & 16.42 & 36.59 & 262.21 \\
\hline S3_Rubber & 235.8 & 4.19 & 12.2 & 1.63 & 0 & 0 & 6.06 & 16.72 & 242.5 \\
\hline S3_Rubber & 561.9 & 4.13 & 13.8 & 2.55 & 0.75 & 0 & 9.26 & 28.96 & 233.25 \\
\hline S4_Rainforest & 258.3 & 4.05 & 13.0 & 0.76 & 0 & 0 & 11.40 & 17.33 & 258.02 \\
\hline S4_Rainforest & 451.7 & 3.78 & 15.9 & 0.50 & 0 & 0 & 8.24 & 8.77 & 225.68 \\
\hline S4_Rainforest & 250.7 & 4.14 & 15.1 & 2.17 & 0.84 & 0 & 13.06 & 26.01 & 239.31 \\
\hline S4_Rainforest & 351.2 & 3.7 & 15.5 & 1.87 & 0.70 & 0 & 13.68 & 24.21 & 257.94 \\
\hline S4_Oil palm & 345.5 & 4.2 & 14.2 & 0.42 & 0.57 & 0 & 2.83 & 2.53 & 250.62 \\
\hline S4_Oil palm & 179.2 & 4.63 & 13.6 & 0.80 & 0 & 0 & 2.06 & 5.51 & 265.88 \\
\hline S4_Oil palm & 98.8 & 4.38 & 12.6 & 0 & 0 & 0 & 0.52 & 0.16 & 267.07 \\
\hline S4_Oil palm & 308.3 & 4.53 & 16.3 & 0.63 & 0.51 & 0 & 8.27 & 12.25 & 255.81 \\
\hline S4_Rubber & 450.1 & 4.2 & 10.9 & 0.43 & 0.86 & 0 & 3.21 & 3.94 & 259.38 \\
\hline S4_Rubber & 267.1 & 4.16 & 11.1 & 0.98 & 0 & 0 & 4.73 & 6.58 & 236.05 \\
\hline S4_Rubber & 227.9 & 4.28 & 11.6 & 0.45 & 0 & 0 & 4.04 & 2.85 & 243.87 \\
\hline S4_Rubber & 455.2 & 4.05 & 13.7 & 0.89 & 0 & 0 & 3.24 & 9.32 & 249.35 \\
\hline
\end{tabular}


Chapter 5

Appendix 5.3. Species matrix of Collembola in the litter layer in rainforest, rubber and oil palm plantations at four sampling dates (S1-March, S2-June, S3-

August, S4-November)

\begin{tabular}{|c|c|c|c|c|c|c|c|c|c|c|c|c|c|c|c|c|c|c|c|c|c|c|c|}
\hline System & Acr & Asc & Folc & Folp & Foln & Hom & Isolu & Isomi & Isosy & Lep & Meg & Par & Pro & Psed & Pseu & Pte & Ram & Sal & Sm.g & Sph1 & Sph2 & Sphy & Tha \\
\hline S1_Rainforest & 0 & 0 & 7 & 72 & 8 & 0 & 0 & 24 & 7 & 0 & 31 & 0 & 0 & 0 & 40 & 0 & 1 & 0 & 15 & 30 & 0 & 0 & 0 \\
\hline S1_Rainforest & 0 & 32 & 0 & 2 & 0 & 0 & 0 & 15 & 10 & 0 & 13 & 0 & 0 & 0 & 95 & 0 & 2 & 0 & 25 & 0 & 0 & 0 & 0 \\
\hline S1_Rainforest & 0 & 29 & 0 & 1 & 14 & 0 & 19 & 13 & 0 & 0 & 39 & 3 & 1 & 0 & 178 & 0 & 1 & 0 & 10 & 10 & 11 & 0 & 16 \\
\hline S1_Rainforest & 1 & 26 & 2 & 7 & 13 & 0 & 9 & 0 & 0 & 0 & 0 & 7 & 0 & 0 & 6 & 0 & 4 & 0 & 0 & 4 & 0 & 0 & 5 \\
\hline S1_Oil palm & 1 & 0 & 19 & 3 & 0 & 0 & 6 & 0 & 0 & 1 & 0 & 0 & 0 & 1 & 4 & 3 & 0 & 0 & 0 & 0 & 0 & 0 & 0 \\
\hline S1_Oil palm & 0 & 0 & 11 & 0 & 0 & 2 & 0 & 0 & 0 & 0 & 0 & 0 & 0 & 0 & 0 & 0 & 0 & 0 & 0 & 0 & 0 & 0 & 0 \\
\hline S1_Oil palm & 0 & 1 & 4 & 3 & 0 & 0 & 4 & 0 & 0 & 0 & 0 & 0 & 0 & 0 & 0 & 1 & 0 & 1 & 0 & 0 & 0 & 0 & 0 \\
\hline S1_Oil palm & 0 & 0 & 5 & 0 & 0 & 1 & 1 & 0 & 0 & 0 & 0 & 0 & 0 & 0 & 0 & 1 & 0 & 0 & 0 & 0 & 0 & 0 & 0 \\
\hline S1_Rubber & 0 & 0 & 1 & 1 & 0 & 3 & 0 & 0 & 0 & 0 & 0 & 0 & 0 & 0 & 0 & 0 & 0 & 1 & 0 & 0 & 0 & 0 & 0 \\
\hline S1_Rubber & 0 & 0 & 10 & 1 & 0 & 0 & 7 & 0 & 0 & 3 & 0 & 0 & 0 & 0 & 1 & 0 & 0 & 0 & 3 & 0 & 0 & 5 & 0 \\
\hline S1_Rubber & 0 & 0 & 19 & 3 & 0 & 3 & 0 & 0 & 0 & 0 & 0 & 0 & 0 & 0 & 0 & 0 & 0 & 0 & 0 & 0 & 0 & 0 & 0 \\
\hline S1_Rubber & 0 & 3 & 4 & 3 & 0 & 0 & 1 & 0 & 0 & 0 & 0 & 0 & 0 & 0 & 1 & 0 & 0 & 0 & 0 & 2 & 0 & 0 & 0 \\
\hline S2_Rainforest & 0 & 1 & 0 & 0 & 0 & 0 & 0 & 0 & 0 & 0 & 0 & 0 & 0 & 0 & 0 & 0 & 0 & 0 & 0 & 0 & 0 & 0 & 0 \\
\hline S2_Rainforest & 1 & 92 & 3 & 6 & 1 & 0 & 9 & 0 & 0 & 0 & 59 & 0 & 0 & 14 & 114 & 0 & 53 & 0 & 9 & 5 & 4 & 13 & 1 \\
\hline S2_Rainforest & 0 & 0 & 15 & 5 & 5 & 0 & 4 & 0 & 5 & 1 & 19 & 0 & 0 & 0 & 95 & 0 & 0 & 1 & 5 & 9 & 0 & 3 & 6 \\
\hline S2_Oil palm & 0 & 0 & 41 & 10 & 0 & 0 & 5 & 4 & 0 & 0 & 0 & 0 & 0 & 0 & 11 & 4 & 0 & 0 & 2 & 2 & 0 & 0 & 3 \\
\hline S2_Oil palm & 0 & 0 & 61 & 1 & 0 & 0 & 0 & 0 & 0 & 0 & 0 & 0 & 0 & 0 & 0 & 1 & 0 & 0 & 2 & 0 & 0 & 0 & 0 \\
\hline S2_Oil palm & 21 & 0 & 49 & 5 & 0 & 1 & 0 & 0 & 0 & 0 & 0 & 0 & 0 & 0 & 6 & 10 & 0 & 0 & 12 & 0 & 0 & 0 & 0 \\
\hline S2_Oil palm & 0 & 0 & 9 & 0 & 0 & 0 & 2 & 0 & 0 & 0 & 0 & 0 & 0 & 0 & 3 & 0 & 0 & 0 & 0 & 0 & 0 & 0 & 1 \\
\hline S2_Rubber & 0 & 5 & 0 & 0 & 0 & 0 & 3 & 0 & 0 & 0 & 4 & 0 & 0 & 0 & 4 & 0 & 0 & 0 & 2 & 0 & 0 & 0 & 1 \\
\hline S2_Rubber & 5 & 7 & 6 & 1 & 0 & 0 & 2 & 0 & 0 & 0 & 0 & 0 & 0 & 0 & 2 & 2 & 0 & 0 & 9 & 1 & 6 & 1 & 0 \\
\hline S2_Rubber & 0 & 7 & 14 & 0 & 0 & 1 & 0 & 0 & 0 & 0 & 0 & 0 & 0 & 0 & 0 & 1 & 0 & 0 & 5 & 2 & 0 & 0 & 0 \\
\hline S2_Rubber & 0 & 45 & 19 & 3 & 0 & 0 & 11 & 21 & 0 & 0 & 24 & 1 & 3 & 0 & 19 & 1 & 0 & 0 & 10 & 7 & 0 & 1 & 0 \\
\hline S3_Rainforest & 1 & 7 & 39 & 73 & 0 & 0 & 0 & 0 & 0 & 0 & 3 & 2 & 0 & 12 & 45 & 0 & 4 & 0 & 0 & 216 & 0 & 31 & 0 \\
\hline S3_Rainforest & 0 & 24 & 0 & 22 & 0 & 0 & 32 & 0 & 0 & 0 & 17 & 0 & 0 & 0 & 33 & 0 & 0 & 2 & 0 & 35 & 0 & 0 & 0 \\
\hline S3_Rainforest & 0 & 39 & 1 & 20 & 0 & 0 & 20 & 0 & 0 & 0 & 1 & 0 & 0 & 6 & 20 & 0 & 0 & 0 & 0 & 10 & 10 & 6 & 2 \\
\hline S3_Oil palm & 1 & 14 & 0 & 0 & 0 & 0 & 0 & 0 & 0 & 0 & 2 & 3 & 0 & 0 & 6 & 0 & 0 & 0 & 0 & 10 & 13 & 0 & 0 \\
\hline
\end{tabular}


Chapter 5

\begin{tabular}{|c|c|c|c|c|c|c|c|c|c|c|c|c|c|c|c|c|c|c|c|c|c|c|c|}
\hline S3_Oil palm & 0 & 0 & 0 & 0 & 0 & 0 & 0 & 0 & 0 & 0 & 0 & 0 & 0 & 0 & 0 & 0 & 0 & 0 & 0 & 0 & 0 & 1 & 0 \\
\hline S3_Oil palm & 0 & 0 & 0 & 0 & 0 & 0 & 0 & 0 & 0 & 0 & 0 & 0 & 0 & 0 & 0 & 0 & 0 & 0 & 0 & 0 & 0 & 0 & 2 \\
\hline S3_Oil palm & 0 & 0 & 1 & 0 & 0 & 0 & 0 & 0 & 0 & 0 & 0 & 0 & 0 & 0 & 0 & 0 & 0 & 0 & 3 & 0 & 0 & 0 & 0 \\
\hline S3_Rubber & 0 & 0 & 1 & 0 & 0 & 0 & 0 & 0 & 0 & 1 & 0 & 0 & 0 & 0 & 0 & 0 & 0 & 0 & 4 & 0 & 0 & 0 & 0 \\
\hline S3_Rubber & 0 & 31 & 0 & 0 & 0 & 0 & 17 & 0 & 10 & 0 & 6 & 26 & 0 & 5 & 14 & 0 & 0 & 0 & 0 & 0 & 4 & 0 & 0 \\
\hline S3_Rubber & 0 & 4 & 28 & 19 & 0 & 0 & 7 & 0 & 0 & 8 & 0 & 6 & 0 & 0 & 0 & 3 & 0 & 0 & 0 & 9 & 19 & 28 & 1 \\
\hline S3_Rubber & 0 & 6 & 4 & 1 & 2 & 0 & 8 & 3 & 0 & 0 & 1 & 0 & 1 & 0 & 66 & 0 & 8 & 0 & 0 & 0 & 0 & 0 & 0 \\
\hline S4_Rainforest & 0 & 7 & 0 & 0 & 0 & 21 & 0 & 0 & 0 & 0 & 0 & 0 & 0 & 0 & 0 & 0 & 0 & 4 & 0 & 1 & 0 & 0 & 0 \\
\hline S4_Rainforest & 0 & 2 & 3 & 6 & 0 & 0 & 0 & 15 & 7 & 2 & 21 & 2 & 1 & 0 & 61 & 0 & 11 & 0 & 39 & 0 & 43 & 0 & 1 \\
\hline S4_Rainforest & 0 & 69 & 3 & 6 & 2 & 0 & 0 & 0 & 18 & 0 & 29 & 11 & 0 & 0 & 63 & 0 & 5 & 1 & 8 & 0 & 17 & 0 & 0 \\
\hline S4_Rainforest & 0 & 28 & 4 & 0 & 0 & 0 & 0 & 17 & 0 & 16 & 30 & 5 & 0 & 4 & 30 & 0 & 1 & 0 & 0 & 68 & 0 & 0 & 0 \\
\hline S4_Oil palm & 0 & 43 & 4 & 2 & 0 & 0 & 0 & 0 & 11 & 0 & 6 & 2 & 0 & 1 & 31 & 0 & 7 & 0 & 0 & 53 & 0 & 8 & 0 \\
\hline S4_Oil palm & 0 & 0 & 75 & 6 & 0 & 0 & 2 & 0 & 0 & 0 & 3 & 0 & 0 & 0 & 7 & 0 & 0 & 0 & 0 & 1 & 0 & 0 & 0 \\
\hline S4_Oil palm & 0 & 1 & 50 & 2 & 0 & 0 & 1 & 0 & 0 & 0 & 2 & 0 & 0 & 0 & 9 & 0 & 5 & 4 & 0 & 0 & 0 & 0 & 0 \\
\hline S4_Oil palm & 0 & 0 & 10 & 0 & 0 & 0 & 10 & 0 & 0 & 2 & 0 & 0 & 0 & 0 & 1 & 0 & 0 & 0 & 0 & 0 & 0 & 0 & 0 \\
\hline S4_Rubber & 0 & 1 & 45 & 10 & 0 & 0 & 0 & 0 & 0 & 2 & 0 & 0 & 0 & 1 & 23 & 2 & 0 & 0 & 0 & 1 & 0 & 2 & 0 \\
\hline S4_Rubber & 0 & 1 & 14 & 0 & 0 & 0 & 0 & 76 & 44 & 6 & 54 & 0 & 0 & 3 & 3 & 0 & 0 & 0 & 0 & 3 & 0 & 0 & 2 \\
\hline S4_Rubber & 2 & 1 & 16 & 47 & 0 & 1 & 0 & 0 & 8 & 3 & 1 & 0 & 0 & 0 & 0 & 2 & 0 & 0 & 0 & 0 & 1 & 0 & 1 \\
\hline S4_Rubber & 0 & 14 & 4 & 0 & 0 & 1 & 0 & 56 & 0 & 0 & 40 & 0 & 3 & 0 & 31 & 0 & 5 & 0 & 0 & 0 & 0 & 0 & 23 \\
\hline
\end{tabular}

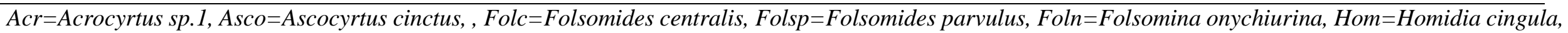

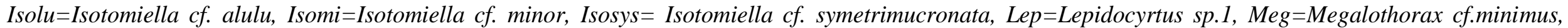

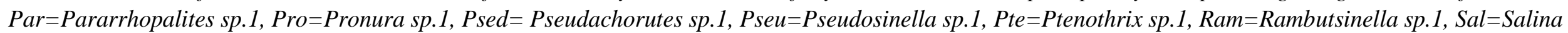
sp.1, Sm.g=Sminthurides sp.1, Spha= Sphaeridia sp.1, Spha=Sphaeridia sp.2, Sphy= Sphyroteca sp.1, Xen=Xenylla sp.1 
Appendix 5.4. Species matrix of Collembola in the litter layer in rainforest, rubber and oil palm plantations at four sampling dates (S1-March, S2-June, S3August, S4-November)

\begin{tabular}{|c|c|c|c|c|c|c|c|c|c|c|c|c|c|c|}
\hline System & Acr & All & Asc & Folc & Folp & Foln & Hom & Isolu & Isodes & Meg & Pseu & Ram & Sphy & Xen \\
\hline S1_Rainforest & 0 & 6 & 0 & 0 & 1 & 0 & 0 & 3 & 0 & 3 & 12 & 0 & 0 & 1 \\
\hline S1_Rainforest & 0 & 15 & 3 & 0 & 0 & 0 & 0 & 4 & 0 & 0 & 7 & 0 & 0 & 0 \\
\hline S1_Rainforest & 0 & 10 & 7 & 5 & 0 & 10 & 0 & 0 & 0 & 4 & 69 & 0 & 0 & 3 \\
\hline S1_Rainforest & 0 & 0 & 2 & 0 & 0 & 50 & 0 & 0 & 0 & 0 & 27 & 6 & 0 & 0 \\
\hline S1_Oil palm & 5 & 12 & 0 & 1 & 0 & 0 & 0 & 0 & 1 & 0 & 14 & 0 & 0 & 0 \\
\hline S1_Oil palm & 3 & 0 & 0 & 7 & 0 & 0 & 2 & 0 & 0 & 0 & 11 & 0 & 0 & 1 \\
\hline S1_Oil palm & 1 & 0 & 0 & 3 & 1 & 0 & 0 & 3 & 17 & 0 & 2 & 0 & 0 & 0 \\
\hline S1_Oil palm & 0 & 7 & 0 & 18 & 1 & 4 & 1 & 0 & 0 & 6 & 51 & 1 & 0 & 0 \\
\hline S1_Rubber & 0 & 20 & 14 & 0 & 0 & 0 & 0 & 0 & 0 & 0 & 33 & 0 & 0 & 0 \\
\hline S1_Rubber & 0 & 8 & 49 & 5 & 1 & 0 & 0 & 0 & 0 & 0 & 48 & 0 & 1 & 2 \\
\hline S1_Rubber & 0 & 3 & 1 & 12 & 3 & 2 & 1 & 0 & 0 & 0 & 7 & 0 & 0 & 0 \\
\hline S1_Rubber & 0 & 0 & 6 & 8 & 0 & 0 & 0 & 8 & 0 & 9 & 29 & 3 & 0 & 0 \\
\hline S2_Rainforest & 1 & 1 & 0 & 5 & 0 & 0 & 0 & 4 & 0 & 4 & 70 & 5 & 1 & 0 \\
\hline S2_Rainforest & 0 & 0 & 3 & 0 & 1 & 3 & 0 & 3 & 0 & 13 & 78 & 15 & 1 & 0 \\
\hline S2_Rainforest & 0 & 0 & 7 & 0 & 1 & 9 & 0 & 6 & 0 & 0 & 50 & 0 & 0 & 1 \\
\hline S2_Rainforest & 0 & 0 & 2 & 0 & 0 & 1 & 0 & 0 & 0 & 0 & 17 & 3 & 0 & 1 \\
\hline S2_Oil palm & 10 & 6 & 0 & 1 & 3 & 0 & 0 & 2 & 2 & 3 & 24 & 0 & 0 & 0 \\
\hline S2_Oil palm & 0 & 3 & 0 & 6 & 0 & 0 & 0 & 0 & 0 & 0 & 22 & 0 & 0 & 0 \\
\hline S2_Oil palm & 1 & 0 & 4 & 3 & 0 & 0 & 1 & 0 & 0 & 0 & 41 & 0 & 0 & 0 \\
\hline S2_Oil palm & 0 & 3 & 0 & 4 & 0 & 0 & 0 & 3 & 0 & 0 & 24 & 0 & 0 & 0 \\
\hline S2_Rubber & 0 & 5 & 30 & 0 & 0 & 0 & 0 & 0 & 0 & 19 & 32 & 0 & 2 & 2 \\
\hline S2_Rubber & 0 & 0 & 28 & 5 & 1 & 0 & 0 & 2 & 0 & 5 & 10 & 0 & 0 & 0 \\
\hline S2_Rubber & 2 & 3 & 4 & 4 & 0 & 0 & 0 & 0 & 0 & 0 & 12 & 0 & 0 & 0 \\
\hline S2_Rubber & 0 & 1 & 73 & 6 & 1 & 0 & 0 & 31 & 0 & 38 & 111 & 0 & 0 & 0 \\
\hline S3_Rainforest & 0 & 0 & 27 & 0 & 0 & 0 & 0 & 38 & 0 & 3 & 59 & 23 & 0 & 9 \\
\hline S3_Rainforest & 0 & 0 & 37 & 0 & 3 & 1 & 0 & 0 & 0 & 6 & 92 & 2 & 0 & 3 \\
\hline S3_Rainforest & 0 & 0 & 19 & 0 & 4 & 11 & 0 & 0 & 0 & 10 & 141 & 20 & 1 & 0 \\
\hline S3_Oil palm & 2 & 17 & 0 & 10 & 3 & 0 & 0 & 0 & 1 & 13 & 51 & 12 & 1 & 0 \\
\hline S3_Oil palm & 0 & 0 & 0 & 4 & 0 & 3 & 0 & 0 & 2 & 0 & 31 & 0 & 0 & 0 \\
\hline
\end{tabular}


Chapter 5

\begin{tabular}{lllrrllllllllll}
\hline S3_Oil palm & 1 & 0 & 0 & 3 & 1 & 0 & 0 & 0 & 3 & 0 & 0 & 0 & 0 & 0 \\
S3_Oil palm & 0 & 5 & 55 & 25 & 6 & 2 & 0 & 0 & 0 & 0 & 35 & 0 & 0 & 0 \\
S3_Rubber & 1 & 0 & 81 & 1 & 0 & 0 & 0 & 0 & 0 & 22 & 29 & 0 & 0 & 0 \\
S3_Rubber & 0 & 3 & 74 & 4 & 0 & 0 & 0 & 7 & 0 & 3 & 32 & 0 & 2 & 1 \\
S3_Rubber & 0 & 2 & 0 & 14 & 6 & 0 & 0 & 0 & 0 & 1 & 18 & 0 & 0 & 0 \\
S3_Rubber & 0 & 0 & 40 & 5 & 1 & 0 & 20 & 4 & 0 & 8 & 14 & 5 & 0 & 0 \\
S4_Rainforest & 0 & 1 & 0 & 1 & 0 & 1 & 0 & 0 & 0 & 2 & 41 & 4 & 0 & 0 \\
S4_Rainforest & 0 & 0 & 3 & 0 & 0 & 1 & 0 & 0 & 0 & 1 & 17 & 0 & 0 & 0 \\
S4_Rainforest & 0 & 0 & 2 & 0 & 1 & 0 & 0 & 0 & 1 & 4 & 33 & 0 & 0 & 0 \\
S4_Rainforest & 0 & 0 & 18 & 0 & 0 & 5 & 0 & 0 & 0 & 0 & 22 & 4 & 0 & 0 \\
S4_Oil palm & 7 & 0 & 0 & 1 & 0 & 0 & 0 & 2 & 10 & 0 & 18 & 0 & 0 & 0 \\
S4_Oil palm & 0 & 0 & 0 & 1 & 0 & 0 & 0 & 0 & 0 & 0 & 21 & 2 & 0 & 0 \\
S4_Oil palm & 0 & 0 & 0 & 0 & 0 & 0 & 0 & 0 & 3 & 0 & 0 & 0 & 0 & 0 \\
S4_Oil palm & 0 & 0 & 0 & 1 & 0 & 0 & 0 & 3 & 0 & 3 & 32 & 0 & 0 & 1 \\
S4_Rubber & 0 & 0 & 1 & 0 & 0 & 0 & 0 & 9 & 0 & 25 & 6 & 0 & 0 & 0 \\
S4_Rubber & 0 & 0 & 16 & 1 & 0 & 0 & 0 & 6 & 0 & 16 & 3 & 0 & 0 & 1 \\
S4_Rubber & 0 & 0 & 0 & 1 & 0 & 0 & 0 & 0 & 0 & 0 & 9 & 0 & 0 & 0 \\
\hline ACIACrocyrus
\end{tabular}

Acr=Acrocyrtus sp.1, All=Alloscopus tetracanthus, Asc=Ascocyrtus cinctus, Folc=Folsomides centralis, Folsp=Folsomides parvulus, Foln=Folsomina onychiurina, Hom=Homidia cingula, Isolu=Isotomiella cf. alulu, Isodes=Isotomodes sp.1, Mega=Megalothorax cf.minimus, Pseul=Pseudosinella sp.1, Ram=Rambutsinella sp.1, Sphy= Sphyroteca sp.1, Xen1= Xenylla sp.1 
Appendix 5.5. Model selection for abundance based on system, layer, plot and season

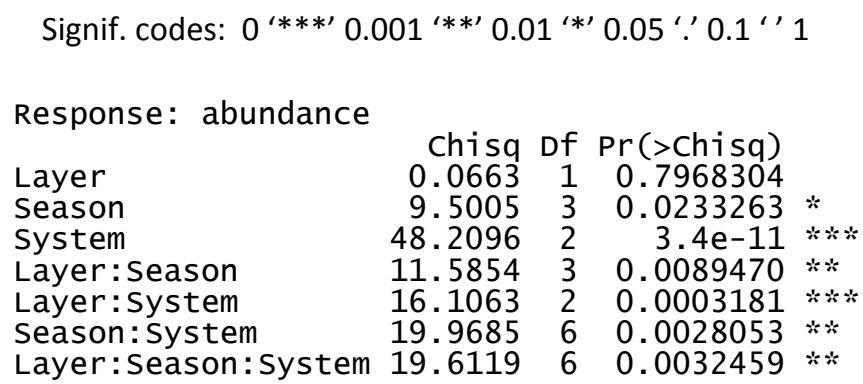

Appendix 5.6. Model selection for abundance based on system, layer, plot, season and life form separately

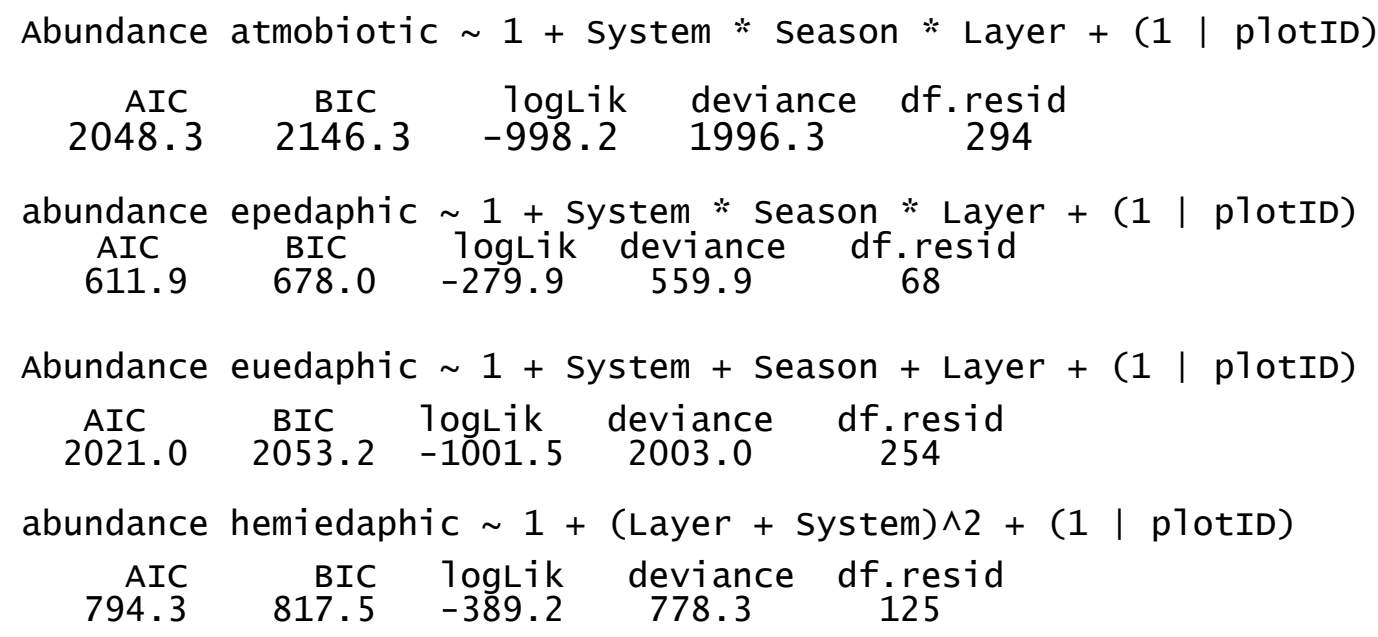


Chapter 5 
CHAPTER 6

GENERAL DISCUSSION
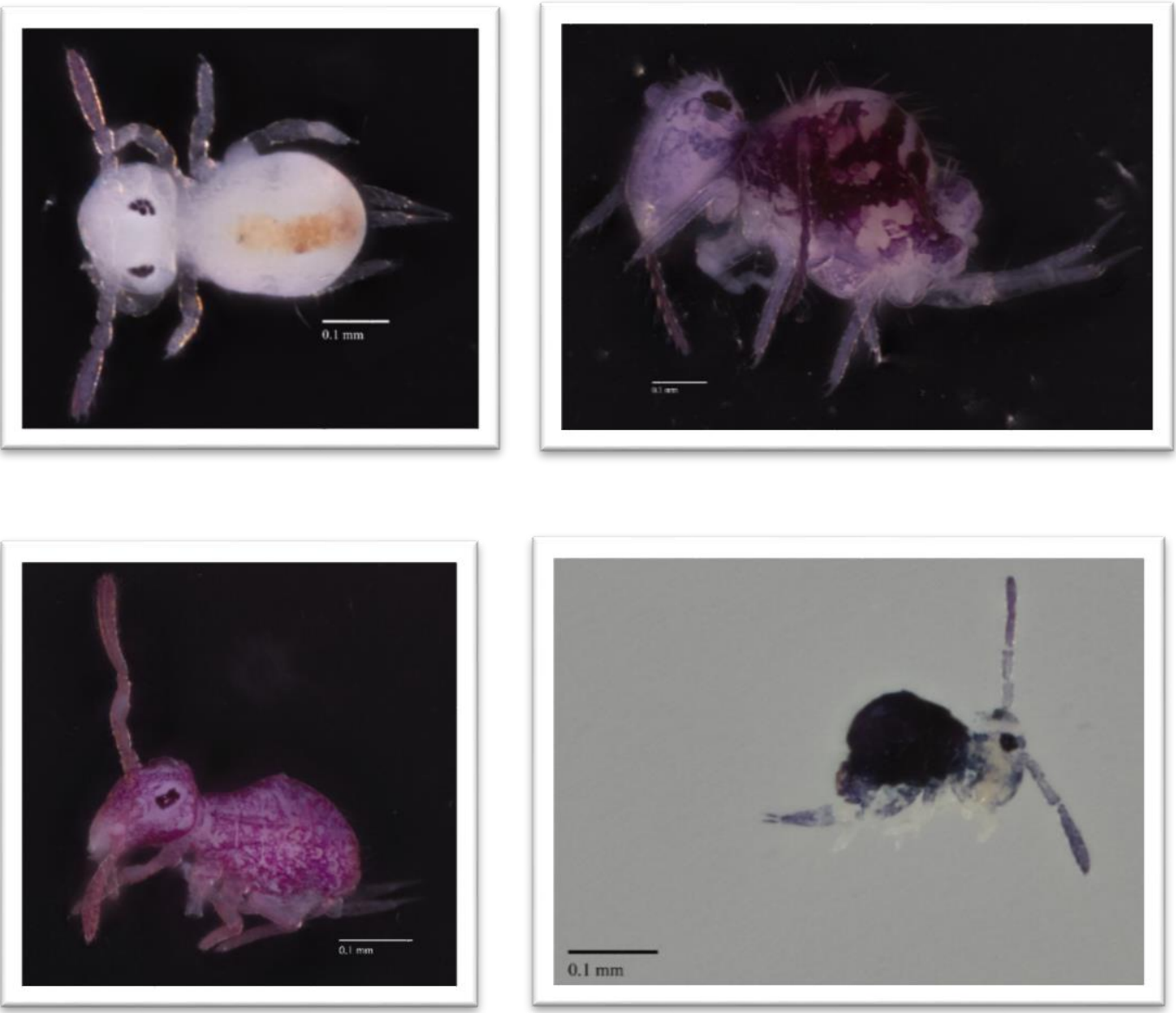

Photos by Winda Ika Susanti 


\section{Chapter 6}

\section{GENERAL DISCUSSION}

Results of this thesis represent major advances in our understanding of the ecological consequences of rainforest conversion into agricultural plantation (rubber and oil palm plantations) in tropical regions of Indonesia. Using three different methods (fatty acid analysis, stable isotope analysis and morphological identification), I showed that rainforest conversion affects resource use in soil animal food webs and changes the taxonomic and functional composition as well as trophic differentiation in a dominant group of soil microarthropods - Collembola. The changes were more pronounced in litter than in soil and in oil palm plantations compared to rubber plantations. The following discussion of the results follows the main hypotheses as presented in the Introduction. Overall, the results suggest that conversion of rainforest into agricultural plantations change soil food webs, with the plant energy channel being more important in plantations (Hypothesis 1). Rainforest conversion also changes density, diversity and community composition of litter and soil Collembola (Hypothesis 2) and trophic niche differentiation of Collembola (Hypothesis 3) with environmental factors and seasonal fluctuations differentially affecting density and community composition among land-use systems (Hypothesis 4).

Hypothesis 1: Soil food webs change in response to the conversion of rainforest into agricultural plantations, with the bacterial energy channel being more important in rainforest and the plant energy channel being more important in plantations.

The way energy is channeled through soil food webs is a major determinant of their stability (Moore and De Ruiter, 2012). Food web models allow calculating energy and nutrient fluxes based on consumer - resource interactions, and the way how energy and nutrients are channeled through soil microbial and animal communities (De Ruiter et $a l .$, 1993). Rainforest conversion is likely to alter the flux of energy through soil food webs. This channeling has been shown to be altered significantly with rainforest conversion. The changes of chanelling in soil food web stuctures as effects of land-use intensification is in line with the study in temperate ecosystems (De Vries et al., 2013). The results of neutral lipid fatty acid (NLFA) analysis (Chapter 2) confirmed Hypothesis 1 of my thesis that the channeling of energy through soil food webs differs between the three land-use systems studied (rainforest, rubber and oil palm plantations). NLFA analysis indicated that soil animal food webs in oil palm plantations indeed more heavily 
rely on plant resources, whereas animal food webs in rainforest more heavily rely on Gram-positive and Gram-negative bacteria. The results of Chapter 2 are in line with results of the study of Pollierer et al. (2015) from temperate forests reporting that forest types / management practices alter microbial community composition and as a consequence the relative strength of the fungal and bacterial energy channel. In addition, the study by De Vries et al. (2013) reported the bacterial energy channel to be reduced by intensive land-use.

The results from Chapter 2 also suggested that, based on NLFA analysis, soil and litter Collembola are using algae as important food resources in addition to fungi and litter. This is consistent with findings reported in Chapter 4 based on stable isotope analysis that certain species of soil and litter Collembola, in particular Paronellidae, to a substantial extent feed on algae/non-vascular plants (Susanti et al., 2021). Generally, the results presented in Chapter 4 suggest that the trophic position of and use of food resources by Collembola in tropical forest ecosystems resemble those of Collembola in temperate forest ecosystems (Pollierer et al., 2009; Korotkevich et al., 2018). Overall, these results suggest that Collembola rely little on fresh plant material or mycorrhizal fungi as food in both temperate and tropical ecosystems, and this may well apply to soil food webs in general (Potapov et al., 2018). Corresponding to the results presented in Chapter 2, low $\delta^{15} \mathrm{~N}$ values were attributed to epedaphic and atmobiotic Collembola feeding on algae and/or lichens, which is widespread in Collembola in temperate forests (Potapov et al., 2018). This pattern applied e.g., to some species of Paronellidae (Salina sp.1, Callyntrura sp.1 and Lepidonella sp.1) in rainforest and jungle rubber with $\delta^{15} \mathrm{~N}$ values below those of leaf litter suggesting algivory. However, in the study presented in Chapter 4 only few species had $\delta^{15} \mathrm{~N}$ values below those of litter contradicting results based on fatty acid analysis (Chapter 2) and suggesting that Collembola in tropical ecosystems feed more on algae than in temperate ecosystems (Susanti et al., 2019). This may be due to Paronellidae having $\delta^{15} \mathrm{~N}$ values below those of litter as presented in Chapter 4. Overall, the results from fatty acid and stable isotope analysis confirmed Hypothesis 1 that rainforest conversion affects the channeling of energy through soil food webs with the channeling in rainforest being based more on bacteria, whereas in plantations it is more strongly based on plants. The results presented in Chapter 2 also suggest that the conversion of rainforest into plantations is associated with pronounced shifts in the community composition of mesofauna detritivores with potential major consequences for the functioning of the decomposer system. 
Hypothesis 2: Density, species diversity, and functional diversity are higher in rainforest than in plantations system, with species and trait community composition differing between plantation systems.

The analysis of the species and functional composition of Collembola communities presented in Chapter 3 confirmed Hypothesis 2 that rainforest conversion changes the community and trait composition and also negatively impacts density and diversity (including species diversity, functional diversity and functional dispersion) of soil and litter Collembola. Declining Collembola density after rainforest conversion is in line with a decline in plant and animal diversity above (Fitzherbert et al., 2008; Clough et al., 2016; Rembold et al., 2017) as well as below the ground, as shown for nematodes (Krashevska et al., 2019), testate amoebae (Krashevska et al., 2016), ground spiders (Potapov et al., 2020) and litter macroinvertebrates (Barnes et al., 2014). These changes also paralleled with the changes in energy channels of soil food webs after rainforest conversion (Chapter 2), and suggest that these changes at least in part were due to changes in the community composition of soil animal taxa. The high density of Collembola in the soil layer of oil palm plantations presumably was due to the low amount of litter in the litter layer. Changes in functional trait and species composition after rainforest conversion into agricultural plantations were reported in studies on different taxa, e.g. bacteria (Rodrigues et al., 2013), ground spiders (Potapov et al., 2020a) and birds (Edwards et al., 2013). As presented in Chapter 3, rainforest conversion also altered the trait composition of Collembola communities. Trait analysis showed that dominating Collembola species in rainforest and jungle rubber were characterized by small size, absence of furca and absence or intense pigmentation, while larger species with long furca and diffuse or patterned coloration were more abundant in plantations. This trend may explain the dominance of Pseudosinella sp.1 and Isotomiella spp. in rainforest. The larger species with higher number of ocelli and patterned or intensive pigmentation presumably are well adapted to environmental conditions in plantations which is in line with study by Makkonen et al. (2011).

Water content and $\mathrm{pH}$ were identified as environmental factors significantly impacting species and trait composition of Collembola across land-use systems irrespective of the soil layer they colonized (litter and soil). This suggests that water content and $\mathrm{pH}$ are also important factors driving soil animal community composition in the tropics as repeatedly shown for temperate ecosystems. The results of the present study 
are in line with those of Salamon et al. (2008) reporting functional groups/species of Collembola to mainly depend on abiotic factors such as $\mathrm{pH}$ and soil water content. In addition, the study by Pollierer and Scheu (2017) reported that $\mathrm{pH}$ functions as important factor driving Collembola communities. The results presented in Chapter 3 also are in line with the results on the response of Collembola to seasonal variations (Chapter 5), which revealed that water content and $\mathrm{pH}$ are important environmental factors driving Collembola communities. Further, I also detected potential biotic effects of top-down control by micro- and macropredators. Overall, the results on the response of Collembola communities suggest that rainforest conversion affects species and trait composition due to changes in environmental factors driving Collembola community composition. Collembola communities can cope well with environmental changes in general presumably as they are able to respond in a flexible way to changes in the availability of resources and environmental conditions, as also reported for Oribatida (Krause et al., 2021). Collembola are characterized by fast reproduction reaching high abundance short after strong disturbances (González-Macé and Scheu, 2018). Similar to other arthropod taxa (Araneae, Carabidae, Chilopoda, Auchenorrhyncha, Heteroptera, Oribatida) (Birkhofer et al., 2017) trait composition of Collembola significantly responds to changes in land use.

Hypothesis 3: Trophic niche differentiation among Collembola species is more pronounced in rainforest than in plantation systems with Collembola in plantations shifting their trophic niches towards herbivory.

The results of stable isotope analyses of Collembola species and communities presented in Chapter 4 confirmed Hypothesis 3 that rainforest conversion into rubber and oil palm plantations changes trophic position and food resources of soil and litter Collembola. The shift in trophic niches of Collembola was mainly due to changes in the use of basal resources rather than trophic levels (Susanti et al., 2021). Highest $\Delta^{13} \mathrm{C}$ values in rainforest suggest more pronounced processing of litter resources by microorganisms and consumption of these microorganisms by Collembola. In line with the results presented in Chapter 2, lower $\Delta^{13} \mathrm{C}$ values, but high $\Delta{ }^{13} \mathrm{C}$ variation in Collembola in oil palm plantations indicated that Collembola shift towards herbivory and use more variable resources in this system. Small range in $\Delta^{15} \mathrm{~N}$ values in Collembola species in monoculture plantations in comparison to rainforest indicated that conversion of rainforest into plantations is associated with simplification in the trophic structure of 
Collembola communities and this was confirmed by generally lower isotopic niche differentiation among species in plantations. Simplification of food-web structure in disturbed ecosystems also has been reported for Collembola communities in the temperate region (Korotkevich et al., 2018). In line with Collembola , changes in trophic position ( $\delta^{15} \mathrm{~N}$ values) as well as changes in the use of basal resources ( $\delta^{13} \mathrm{C}$ values) with the shift in trophic position towards higher trophic levels in converted ecosystems also has been reported for Oribatida at the same study sites (Krause et al., 2019). Oribatid mites shifted their diet toward predation and/or scavenging and toward the plant-based energy channel with conversion of rainforest into rubber and oil palm plantations (Krause et al., 2021).

Results presented in Chapter 4 revealed that across the studied ecosystems, atmobiotic Collembola species (Symphypleona and Paronellidae) occupied the lowest, whereas euedaphic species occupied the highest trophic position, resembling patterns in temperate forests (Chahartaghi et al., 2005; Potapov et al., 2016; Potapov et al., 2018). Paronellidae are well adapted to aboveground life, are large-sized and possess welldeveloped visual systems. Such 'atmobiotic' Collembola have been assumed to live at least in part as herbivores on vascular plants or algae (Rusek, 2007). Paronellidae predominantly occur in tropical regions and this suggests that habitat characteristics affect the feeding preferences of Collembola across different phylogenetic lineages and climate types.

Generally, both fatty acid (Chapter 2) and stable isotope analysis (Chapter 4) confirmed that conversion of rainforest into plantation systems changes energy fluxes through soil food webs, which at least in part is due to changes in trophic position and use of food resources by soil and litter Collembola and other soil invertebrates. Indeed, this also has been shown to be the case in Oribatida (Krause et al., 2019) and Chilopoda (Klarner et al., 2017) at our field sites. This indicates that altered trophic niche differentiation and utilization of food resources after rainforest conversion is evident across different taxa and trophic levels in soil food webs. Moreover, stable isotope values of species can be considered as traits (Potapov et al., 2016b) that change with land use. Trophic flexibility which was revealed in Chapter 4 can explain high density of Collembola in oil palm plantations, particularly in the soil layer (Chapter 3). Overall, the results presented in Chapter 4 suggest that rainforest conversion into plantation systems is associated with marked shifts in the structure of trophic niches in soil and litter Collembola with potential consequences for ecosystem functioning and food-web 
stability. The changes in trophic niches of communities indicate trophic plasticity and the ability to shift both their trophic level and the use of basal resources resulting in the simplification of soil food web structure.

Hypothesis 4: Dry season has lower Collembola density and more pronounced differences in community composition among land-use systems.

Confirming Hypothesis 4, community composition of Collembola in rainforest and plantations showed significant seasonal fluctuations with the lowest density of Collembola and more pronounced differences in community composition among landuse systems in the dry season (Chapter 5). Collembola density in the litter layer was at a maximum early in the wet season, whereas in soil the density of Collembola was low in the wet season. The results are in line with previous studies in tropical ecosystems (Reddy et al., 1994; Wiwatwitaya and Takeda, 2005), which revealed that Collembola density is higher in the wet season. In the dry season, differences in community composition among land-use systems were more pronounced, presumably due to the decline of some species of Collembola at unfavorable environmental conditions. Notably, euedaphic species were less affected by seasonal fluctuations in climate than hemi- and epedaphic species and dominated in each of the land-use systems. In fact, lower temporal fluctuation in density of euedaphic species may explain the dominance of euedaphic species at our study sites (Chapter 3), reflecting that by living deeper in soil they are more protected against climatic adversities (Chapter 5). Connecting also with Chapter 4, Pseudosinella sp.1 as euedaphic species dominated across land-use systems and had the highest $\Delta^{15} \mathrm{~N}$ values in most of the land-use systems, suggesting that this species at least in part lives as predator or scavenger.

Additionally, the results presented in Chapter 5 also are in line with those presented in Chapter 3 reporting that water content (as well as $\mathrm{pH}$ ) significantly affected the community composition of litter and soil Collembola. This indicates that water content and $\mathrm{pH}$ are important driving factors for seasonal fluctuations in Collembola, which is in line with studies in temperate ecosystems (Ponge, 1983; Fujii et al., 2014). Water content was an important factor for community composition of Collembola in the wet season, particularly in rainforest, whereas $\mathrm{pH}$ was an important factor for Collembola community composition in the dry season, particularly in plantation systems (Chapter 3 and 5). High water content in rainforest correlated positively with the density of bacteria, 
whereas high $\mathrm{pH}$ in plantation systems correlated positively with the density of mycorrhizal fungi. Further, the density of bacteria also was identified as important driving factor of Collembola communities in rainforest (Chapter 3 and 5), connecting results from fatty acid analysis, which revealed that in rainforest energy is predominantly channeled via the bacterial channel (Chapter 2). Overall, water content was identified as important factor driving Collembola density and community in the wet season, which fits well with the general decline in Collembola density in the dry season.

\section{Overall conclusions}

My thesis provided major insights into the effects of rainforest conversion into agricultural plantations on the structure and food relationships in belowground communities. First, I showed that rainforest conversion into agricultural plantations affects the channeling of energy in soil food webs with a strengthening of the plant energy channel in plantation systems. Further, I showed that rainforest conversion into agricultural plantations negatively impacts litter and soil Collembola, one of the dominant and functionally important group of soil fauna serving as indicator of soil health. For this group, I documented changes in density, community composition, functional composition and trophic niches with conversion of rainforest into plantation systems. Collembola in the litter layer were more affected by the conversion of rainforest into plantatiosn than in the soil layer reflecting the buffering function of soil. Specific traits, such as larger size, patterned or intensive pigmentation and number of ocelli may explain the adaptation of Collembola species to the environmental changes with water content and $\mathrm{pH}$ being the most important driving factors. A dominant species of the euedaphic life form and of high trophic position (Pseudosinella sp.1) was found in high abundance across rainforest and plantations suggesting that this species is best adapted to the studied land-use changes. Aditionally, euedaphic species generally are better adapted to cope with seasonal fluctuations than other life forms. Overall, to reduce the negative impact of rainforest conversion into plantation systems as revealed in this thesis, improved management of agricultural plantations is needed. 


\section{REFERENCES}

A'Bear, A. D., Boddy, L. and Hefin Jones, T. (2012) 'Impacts of elevated temperature on the growth and functioning of decomposer fungi are influenced by grazing collembola', Global Change Biology, 18(6), pp. 1823-1832. doi: 10.1111/j.13652486.2012.02637.x.

Van Aarle, I. M., Olsson, P. A. and Söderström, B. (2002) 'Arbuscular mycorrhizal fungi respond to the substrate $\mathrm{pH}$ of their extraradical mycelium by altered growth and root colonization', New Phytologist, 155(1), pp. 173-182. doi: 10.1046/j.14698137.2002.00439.x.

Adams, E. C. G. (1979) 'On the feeding methods and fine structure of the mouth-parts of Ceratrimeria leleupi Salmon and Adams (Collembola: Neanuridae)', Zoology Publication from Victoria University of Wellington (72).

Aira, M., Monroy, F. and Dominguez, J. (2003) 'Effects of two species of earthworms (AllRolobophora spp.) on soil systems: a microfaunal and biochemical analysisThe 7th international symposium on earthworm ecology ' Cardiff · Wales · 2002', Pedobiologia, 47(5-6), pp. 877-881. doi: 10.1016/S0031-4056(04)70283-8.

Albro, P. W., Schroeder, J. L. and Corbett, J. T. (1992) 'Lipids of the earthworm Lumbricus terrestris.', Lipids, 27(2), pp. 136-43. Available at: http://www.ncbi.nlm.nih.gov/pubmed/8398080.

Allen, K. et al. (2015) 'Soil nitrogen-cycling responses to conversion of lowland forests to oil palm and rubber plantations in Sumatra, Indonesia', PLOS ONE, 10(7), pp. 1-21. doi: 10.1371/journal.pone.0133325.

Allen, K. et al. (2016) 'Spatial variability surpasses land-use change effects on soil biochemical properties of converted lowland landscapes in Sumatra, Indonesia', Geoderma. Elsevier B.V., 284, pp. 42-50. doi: 10.1016/j.geoderma.2016.08.010.

Ambarish, C. N. and Sridhar, K. R. (2015) 'Biochemical composition of two giant pillmillipedes of the Western Ghats of India', Biological Letters, 52(1-2), pp. 45-61. doi: 10.1515/biolet-2015-0014.

André, H. M., Ducarme, X. and Lebrun, P. (2002) 'Soil biodiversity: Myth, reality or conning?', Oikos, 96(1), pp. 3-24. doi: 10.1034/j.1600-0706.2002.11216.x.

Ashwini, K. M. and Sridhar, K. R. (2005) 'Leaf litter preference and conversion by a saprophagous tropical pill millipede , Arthrosphaera magna Attems', 49. doi: 10.1016/j.pedobi.2005.02.002.

Bardgett, R. D. and Van Der Putten, W. H. (2014) 'Belowground biodiversity and ecosystem functioning', Nature. Nature Publishing Group, 515(7528), pp. 505-511. doi: 10.1038 /nature13855.

Barnes, A. D. et al. (2014) 'Consequences of tropical land use for multitrophic biodiversity and ecosystem functioning', Nature Communications. Nature Publishing Group, 5, pp. 1-7. doi: 10.1038/ncomms6351.

Barrios, E. (2007) 'Soil biota, ecosystem services and land productivity', Ecological Economics, 64(2), pp. 269-285. doi: 10.1016/j.ecolecon.2007.03.004.

Bates, D. et al. (2015) 'Fitting Linear Mixed-Effects Models Using 1me4', Journal of 
Statistical Software, 67(1), pp. 1-48. doi: 10.18637jjs.v067.i01.

Birkhofer, K. et al. (2017) 'Land-use type and intensity differentially filter traits in above- and below-ground arthropod communities', Journal of Animal Ecology, 86(3), pp. 511-520. doi: 10.1111/1365-2656.12641.

De Boer, T. E. et al. (2012) 'The influence of long-term copper contaminated agricultural soil at different $\mathrm{pH}$ levels on microbial communities and springtail transcriptional regulation', Environmental Science and Technology, 46(1), pp. 60-68. doi: 10.1021/es2013598.

Bokhorst, S. et al. (2012) 'Extreme winter warming events more negatively impact small rather than large soil fauna: shift in community composition explained by traits not taxa', pp. 1152-1162. doi: 10.1111/j.1365-2486.2011.02565.x.

Bolan, N. S. (1991) 'A critical review on the role of mycorrizal fungi in the uptake of phosphorus by plants', Plant Soil, 134, pp. 189-200.

Bonkow, M., Scheu, S. and Schaefer, M. (1998) 'in a Beechwood on a Basalt Hill : Implications for Litter Decomposition and Soil Formation', 9, pp. 161-166.

Bonkowski, M. and Schaefer, M. (1997) 'interactions between earthworms and soil protozoa : a trophic component in the soil food web', 29(314), pp. 499-502.

ter Braak, C. J. and Smilauer, P. (2012) 'Canoco reference manual and user's guide: software for ordination, version 5.0.'

Brose, U. and Scheu, S. (2014) 'Into darkness: Unravelling the structure of soil food webs', Oikos, 123(10), pp. 1153-1156. doi: 10.1111/oik.01768.

Brückner, A. et al. (2018) 'Imprinted or innate food preferences in the model mite Archegozetes', Soil Organisms, 90(April), pp. 23-26.

Buse, T., Ruess, L. and Filser, J. (2013) 'New trophic biomarkers for Collembola reared on algal diets', Pedobiologia. Elsevier GmbH., 56(3), pp. 153-159. doi: 10.1016/j.pedobi.2013.03.005.

Buse, T., Ruess, L. and Filser, J. (2014) 'Collembola gut passage shapes microbial communities in faecal pellets but not viability of dietary algal cells', Chemoecology, 24(2), pp. 79-84. doi: 10.1007/s00049-013-0145-y.

Cárcamo, H. A. et al. (2000) 'Influence of millipedes on litter decomposition, N mineralization, and microbial communities in a coastal forest in British Columbia, Canada', Canadian Journal of Forest Research, 30(5), pp. 817-826. doi: 10.1139/cjfr30-5-817.

Cavelier, J. and Tobler, A. (2000) 'The effect of abandoned plantations of Pinus patula and Cupressus lusitanica on soils and regeneration of a tropical montane rain forest in Colombia', 347(1998), pp. 335-347.

Chagnon, M. et al. (2001) 'Effects of experimental liming on collembolan communities and soil microbial biomass in a southern quebec sugar maple (Acer saccharum marsh.) stand', Applied Soil Ecology, 17(1), pp. 81-90. doi: 10.1016/S0929-1393(00)00134-7.

Chagnon, M., Hébert, C. and Paré, D. (2000) 'Community structures of Collembola in sugar maple forests: Relations to humus type and seasonal trends', Pedobiologia, 44(2), pp. 148-174. doi: 10.1078/S0031-4056(04)70035-9. 
Chahartaghi, M. et al. (2005) 'Feeding guilds in Collembola based on nitrogen stable isotope ratios', Soil Biology and Biochemistry, 37(9), pp. 1718-1725. doi: 10.1016/j.soilbio.2005.02.006.

Chahartaghi, M. et al. (2009) 'Resource depletion and colonization: A comparison between parthenogenetic and sexual Collembola species', Pedobiologia, 52(3), pp. 181189. doi: 10.1016/j.pedobi.2008.08.003.

Chamberlain, P. M. et al. (2005) 'Fatty acid composition and change in Collembola fed differing diets: Identification of trophic biomarkers', Soil Biology and Biochemistry, 37(9), pp. 1608-1624. doi: 10.1016/j.soilbio.2005.01.022.

Chauvat, M., Ponge, J. F. and Wolters, V. (2007) 'Humus structure during a spruce forest rotation: Quantitative changes and relationship to soil biota', European Journal of Soil Science, 58(3), pp. 625-631. doi: 10.1111/j.1365-2389.2006.00847.x.

Chauvat, M., Wolters, V. and Dauber, J. (2007) 'Response of collembolan communities to land-use change and grassland succession', Ecography, 30(2), pp. 183-192. doi: 10.1111/j.0906-7590.2007.04888.x.

Chauvat, M., Zaitsev, A. S. and Wolters, V. (2003) 'Successional changes of

Collembola and soil microbiota during forest rotation', Oecologia, 137(2), pp. 269-276. doi: 10.1007/s00442-003-1310-8.

Chen, J. et al. (2001) 'Fatty acid composition and dynamics of selected \rfungal-feeding nematodes and fungi', Comparitive Biochemistry and Physiology Part B, 130, pp. 135144.

Chen, T. et al. (2017) 'Neutral lipid fatty acid composition as trait and constraint in Collembola evolution', (March), pp. 9624-9638. doi: 10.1002/ece3.3472.

Clough, Y. et al. (2016) 'Land-use choices follow profitability at the expense of ecological functions in Indonesian smallholder landscapes', Nature Communications, 7. doi: $10.1038 /$ ncomms 13137.

Coulibaly, S. F. M., Winck, B., Akpa-Vinceslas, M., Mignot, L., Le Gras, M., Forey, E., \& Chauvat, M. (2019) 'Functional assemblages of Collembola determine soil microbial communities and associated functions', Frontiers in Environmental Science, 7(52).

Decaëns, T. et al. (2006) 'The values of soil animals for conservation biology', European Journal of Soil Biology, 42(SUPPL. 1). doi: 10.1016/j.ejsobi.2006.07.001.

Deharveng, L., Bedos, A. and Leksawasdi, P. (1989) 'Diversity in tropical forest soils: the Collembola of Doi Inthanon (Thailand)', 3rd International Seminar on Apterygota.

Devi, W. M., Singh, T. B. and Devi, L. J. (2011) 'Monthly changes of collembolan population under the gradients of moisture, organic carbon and nitrogen contents in a sub-tropical forest soil, Manipur .', 2(12), pp. 10-12.

Devillard, E. et al. (2006) 'Rumen ciliate protozoa contain high concentrations of conjugated linoleic acids and vaccenic acid, yet do not hydrogenate linoleic acid or desaturate stearic acid.', The British journal of nutrition. SUB Gottingen, 96(4), pp. 697-704. doi: 10.1079/BJN20061884.

Domínguez, J., Parmelee, R. W. and Edwards, C. A. (2003) 'Interactions between Eisenia andrei (Oligochaeta) and nematode populations during vermicomposting', 
Pedobiologia, 47(1), pp. 53-60. doi: 10.1078/0031-4056-00169.

Doran, J. W. et al. (2000) 'Soil health and sustainability: managing the biotic component of soil quality Soil health and sustainability : managing the biotic component of soil quality', (June).

Drescher, J. et al. (2016) 'Ecological and socio-economic functions across tropical land use systems after rainforest conversion Ecological and socio-economic functions across tropical land use systems after rainforest conversion', (April). doi:

10.1098/rstb.2015.0275.

Edwards, F. A., Edwards, D. P., Hamer, K. C., Davies, R. G. (2013) 'Impacts of logging and conversion of rainforest to oil palm on the functional diversity of birds in $\mathrm{S}$ undaland', Ibis, 155(2), pp. 313-326.

Edwards, D. P. et al. (2014) 'Selective-logging and oil palm: Multitaxon impacts, biodiversity indicators, and trade-offs for conservation planning', Ecological Applications, 24(8), pp. 2029-2049. doi: 10.1890/14-0010.1.

Eitzinger, B. et al. (2018) 'Testing the validity of functional response models using molecular gut content analysis for prey choice in soil predators', Oikos, 127(7), pp. 915-926. doi: 10.1111/oik.04885.

Ek, H. et al. (1994) 'Extramatrical mycelial growth, biomass allocation and nitrogen uptake in ectomycorrhizal systems in response to collembolan grazing', Applied Soil Ecology, 1(2), pp. 155-169. doi: 10.1016/0929-1393(94)90035-3.

Errington, I. et al. (2018) 'The influence of vegetation and soil properties on springtail communities in a diesel-contaminated soil', Science of the Total Environment. Elsevier B.V., 619-620, pp. 1098-1104. doi: 10.1016/j.scitotenv.2017.11.186.

Fatimah, Cholik, E. and Suhardjono, Y. R. (2012) 'Collembola Permukaan Tanah Kebun Karet , Lampung', Bidang Zoologi Pusat Penelitian Biologi LIPI, 21(2), pp. 1722.

Ferlian, O. et al. (2015) 'Trophic niche differentiation and utilisation of food resources in collembolans based on complementary analyses of fatty acids and stable isotopes', Soil Biology and Biochemistry. Elsevier Ltd, 82, pp. 28-35. doi: 10.1016/j.soilbio.2014.12.012.

Fierer, N., Schimel, J. P. and Holden, P. A. (2003) 'Influence of drying-rewetting frequency on soil bacterial community structure', Microbial Ecology, 45(1), pp. 63-71. doi: $10.1007 / \mathrm{s} 00248-002-1007-2$.

Filser, J. (1995) 'The effect of green manure on the distribution of collembola in a permanent row crop', Biology and Fertility of Soils, 19(4), pp. 303-308. doi: 10.1007/BF00336099.

Filser, J. et al. (2002) 'Long-term dynamics and interrelationships of soil Collembola and microorganisms in an arable landscape following land use change', Geoderma, 105(3-4), pp. 201-221.

Fitzherbert, E. B. et al. (2008) 'How will oil palm expansion affect biodiversity?', Trends in Ecology and Evolution, 23(10), pp. 538-545. doi: 10.1016/j.tree.2008.06.012.

Frampton, G. K. and Brink, P. J. Van Den (2002) 'Influence of cropping on the species 
composition on epigeic Collembola in arable fields', 337, pp. 328-337.

Frith, D. and Frith, C. (1990) 'Seasonality of Litter Invertebrate Populations in an Australian Upland Tropical Rain Forest Author ( s ): Dawn Frith and Clifford Frith Published by: Association for Tropical Biology and Conservation Stable URL: https://www.jstor.org/stable/2388411', Biotropica, 22(2), pp. 181-190.

Fujii, S., Saitoh, S. and Takeda, H. (2014) 'Effects of rhizospheres on the community composition of Collembola in a temperate forest', Applied Soil Ecology. Elsevier B.V., 83(April), pp. 109-115. doi: 10.1016/j.apsoil.2014.03.018.

Gagic, V. et al. (2015) 'Functional identity and diversity of animals predict ecosystem functioning better than species-based indices', Proceedings of the Royal Society B: Biological Sciences, 282(1801). doi: 10.1098/rspb.2014.2620.

Gatto, M., Wollni, M. and Qaim, M. (2015) 'Oil palm boom and land-use dynamics in Indonesia: The role of policies and socioeconomic factors', Land Use Policy. Elsevier Ltd, 46, pp. 292-303. doi: 10.1016/j.landusepol.2015.03.001.

Gilbert, N. (2012) 'Palm-oil boom raises conservation concerns', Nature, 487(7405), pp. 14-15. doi: 10.1038/487014a.

Glime, J. M. and Wagner, D. M. (2017) 'Laboratory Techniques: Preservation and Permanent Mounts. Chapt. 2-4', Bryophyte Ecology, 3(September), pp. 1-18.

González-Macé, O. and Scheu, S. (2018) 'Response of collembola and acari communities to summer flooding in a grassland plant diversity experiment', PLOS ONE, 13(8), pp. 1-18. doi: 10.1371/journal.pone.0202862.

Guillaume, T. et al. (2016) 'Sensitivity and resistance of soil fertility indicators to landuse changes : New concept and examples from conversion of Indonesian rainforest to plantations', Ecological Indicators. Elsevier Ltd, 67, pp. 49-57. doi:

10.1016/j.ecolind.2016.02.039.

Guillaume, T., Damris, M. and Kuzyakov, Y. (2015) 'Losses of soil carbon by converting tropical forest to plantations: Erosion and decomposition estimated by $\delta^{13} \mathrm{C}$, Global Change Biology, 21(9), pp. 3548-3560. doi: 10.1111/gcb.12907.

Harwood, J. L. and Russell, N. J. (1984) 'Distribution of lipids', Lipids in Plants and Microbes, pp. 35-70. doi: 978-94-011-5989-0.

Hassler, E. et al. (2015) 'Soil fertility controls soil-atmosphere carbon dioxide and methane fluxes in a tropical landscape converted from lowland forest to rubber and oil palm plantations', Biogeosciences Discussions, 12(12), pp. 9163-9207. doi: 10.5194/bgd-12-9163-2015.

Hättenschwiler, S. et al. (2011) 'Leaf traits and decomposition in tropical rainforests : revisiting some commonly held views and towards a new hypothesis', pp. 950-965.

Haubert, D. et al. (2004) 'Effects of fungal food quality and starvation on the fatty acid composition of Protaphorura fimata (Collembola)', Comparative Biochemistry and Physiology - B Biochemistry and Molecular Biology, 138(1), pp. 41-52. doi: 10.1016/j.cbpc.2004.02.009.

Haubert, D. et al. (2006) 'Trophic shift of stable isotopes and fatty acids in Collembola on bacterial diets', Soil Biology and Biochemistry, 38(7), pp. 2004-2007. doi: 
10.1016/j.soilbio.2005.11.031.

Hawes, T. C. and Greenslade, P. (2015) 'A note on scale morphology in Collembola', Zootaxa, 3925(4), pp. 594-596. doi: 10.11646/zootaxa.3925.4.8.

Heidemann, K. et al. (2014) 'Free-living nematodes as prey for higher trophic levels of forest soil food webs', Oikos, 123(10), pp. 1199-1211. doi: 10.1111/j.16000706.2013.00872.x.

Hopkin, S. P. (1997) 'Biology of the springtails (Insecta, Collembola)'.

Hothorn, T., Bretz, F. and Westfall, P. (2008) 'Simultaneous inference in general parametric models', Biometrical Journal, 50(3), pp. 346-363. doi:

10.1002/bimj.200810425.

Hutson, B. R. and Veitch, L. G. (1987) 'Densities of Collembola and Acarina in the soil and litter of three indigenous South Australian forests related to layer, site and seasonal differences', Australian Journal of Ecology, 12(3), pp. 239-261. doi: 10.1111/j.14429993.1987.tb00946.x.

Illig, J. et al. (2005) 'Where are the decomposers? Uncovering the soil food web of a tropical montane rain forest in southern Ecuador using stable isotopes $(15 \mathrm{~N})$ ', Journal of Tropical Ecology. Massmann Int. Buchh Gmbh, 21(5), pp. 589-593. doi: 10.1017/S0266467405002646.

Jan Leaps, P. S. (2003) Multivariate Analysis Using CANOCO 4.5, Cambridge University Press.

Kanal, A. (2004) 'Effects of fertilisation and edaphic properties on soil-associated Collembola in crop rotation', Agronomy Research, 2(2), pp. 153-168. Available at: http://agronomy.emu.ee/vol022/p2205.pdf.

Kanters, C., Anderson, I. C. and Johnson, D. (2015) 'Chewing up the wood-wide web: Selective grazing on ectomycorrhizal fungi by collembola', Forests, 6(8), pp. 25602570. doi: $10.3390 / \mathrm{f6082560.}$

Kempson, D., Lloyd, M. and Gheraldi, R. (1963) 'A new extractor for woodland litter', Pedobiologia, 3(1), pp. 1-21.

Klarner, B. et al. (2017) 'Trophic niches, diversity and community composition of invertebrate top predators (Chilopoda) as affected by conversion of tropical lowland rainforest in Sumatra (Indonesia)', PLoS ONE, 12(8). doi:

10.1371/journal.pone.0180915.

Klironomos, J. N. and Kendrick, W. B. (1995) 'Relationships among microartropods, fungi, and their environment', Plant and Soil, 170, pp. 183-197. doi: 10.1007/BF02183066.

Koh, L. P. and Ghazoul, J. (2010) 'Reply to Sloan and Stork: Spatially explicit scenario analysis for reconciling agricultural expansion, forest protection, and carbon conservation in Indonesia', Proceedings of the National Academy of Sciences, 107(45), pp. E172-E172. doi: 10.1073/pnas.1012681107.

Korotkevich, A. Y. et al. (2018) 'Collapse of trophic-niche structure in belowground communities under anthropogenic disturbance', Ecosphere, 9(12). doi:

10.1002/ecs2.2528. 
Kotowska, M. M. et al. (2015) 'Quantifying above- and belowground biomass carbon loss with forest conversion in tropical lowlands of Sumatra (Indonesia)', Global Change Biology, 21(10), pp. 3620-3634. doi: 10.1111/gcb.12979.

Krashevska, V. et al. (2015) 'Impact of tropical lowland rainforest conversion into rubber and oil palm plantations on soil microbial communities', Biology and Fertility of Soils, 51(6), pp. 697-705. doi: 10.1007/s00374-015-1021-4.

Krashevska, V. et al. (2016) 'Changes in Structure and Functioning of Protist (Testate Amoebae) Communities Due to Conversion of Lowland Rainforest into Rubber and Oil Palm Plantations', PloS one, 11(7), p. e0160179. doi: 10.1371/journal.pone.0160179.

Krashevska, V. et al. (2018) 'Micro-decomposer communities and decomposition processes in tropical lowlands as affected by land use and litter type', Oecologia. Springer Berlin Heidelberg, 187(1), pp. 255-266. doi: 10.1007/s00442-018-4103-9.

Krashevska, V. et al. (2019) 'Changes in Nematode Communities and Functional Diversity With the Conversion of Rainforest Into Rubber and Oil Palm Plantations', 7(December), pp. 1-10. doi: 10.3389/fevo.2019.00487.

Krause, A. et al. (2019) 'Shift in trophic niches of soil microarthropods with conversion of tropical rainforest into plantations as indicated by stable isotopes (15N, 13C)', PLoS ONE, 14(10), pp. 1-14. doi: 10.1371/journal.pone.0224520.

Krause, A. et al. (2021) 'Variation in Community-Level Trophic Niches of Soil Microarthropods With Conversion of Tropical Rainforest Into Plantation Systems as Indicated by Stable Isotopes (15N, 13C)', Frontiers in Ecology and Evolution, 9(May), pp. 1-10. doi: 10.3389/fevo.2021.592149.

Kühn, J. et al. (2018) 'Community level lipid profiling of consumers as a tool for soil food web diagnostics', Methods in Ecology and Evolution, 9(5), pp. 1265-1275. doi: 10.1111/2041-210X.12966.

Kuznetsova, K., Brockhoff, P. . and Christensen, R. H. B. (2016) 'lmerTest: Test in Linear Mixed Effects Models. R package version 2.0-33.’ Available at: https://cran.rproject.org/package=lmerTest.

Lattaud, C. et al. (1997) 'Activities of the digestive enzymes in the gut and in tissue culture of a tropical geophagous earthworm, Polypheretima elongata (Megascolecidae)', Soil Biology and Biochemistry, 29(3-4), pp. 335-339. doi: 10.1016/S00380717(96)00021-1.

Laumonier, Y. et al. (2010) 'Eco-floristic sectors and deforestation threats in Sumatra: Identifying new conservation area network priorities for ecosystem-based land use planning', Biodiversity and Conservation, 19(4), pp. 1153-1174. doi: 10.1007/s10531010-9784-2.

Lavelle, P. et al. (1987) 'Adaptive strategies of Pontoscolex corethrurus (Glossoscolecidae, Oligochaeta), a peregrine geophagous earthworm of the humid tropics', Biology and Fertility of Soils, pp. 188-194. doi: 10.1007/bf00256899.

Lavelle, P. et al. (2006) 'Soil invertebrates and ecosystem services', European Journal of Soil Biology, 42(SUPPL. 1). doi: 10.1016/j.ejsobi.2006.10.002.

Lawrence, K. L. and Wise, D. H. (2000) 'Spider predation on forest-floor Collembola and evidence for indirect effects on decomposition', Pedobiologia, 44(1), pp. 33-39. 
doi: 10.1078/S0031-4056(04)70026-8.

Lee, A. K. . et al. (2004) 'The 3-hydroxy fatty acids as biomarkers for quantification and characterization of endotoxins and Gram negative bacteria in atmospheric aerosols in Hongkong.', Atmospheric Environment, 38, pp. 6307-6317.

Lenoir, L. et al. (2007) 'Bottom-up or top-down control in forest soil microcosms? Effects of soil fauna on fungal biomass and $\mathrm{C} / \mathrm{N}$ mineralisation', Biology and Fertility of Soils, 43(3), pp. 281-294. doi: 10.1007/s00374-006-0103-8.

Levings, S. C. and Windsor, D. M. (1984) 'Litter Moisture Content as a Determinant of Litter Arthropod Distribution and Abundance During the Dry Season on Barro Colorado Island, Panama', Biotropica, 16(2), p. 125. doi: 10.2307/2387844.

Li, H. et al. (2006) 'Arbuscular mycorrhizal fungi contribute to phosphorus uptake by wheat grown in a phosphorus-fixing soil even in the absence of positive growth responses', New Phytologist, 172(3), pp. 536-543. doi: 10.1111/j.1469-

8137.2006.01846.x.

Liu, J. et al. (2019) 'Spatial and environmental factors are minor structuring forces in a soil Collembola metacommunity in a maize agroecosystem', Pedobiologia. Elsevier, 76(July), p. 150572. doi: 10.1016/j.pedobi.2019.150572.

Madan, R. et al. (2002) 'Use of fatty acids for identification of AM fungi and estimation of the biomass of AM spores in soil', Soil Biology and Biochemistry, 34(1), pp. 125128. doi: 10.1016/S0038-0717(01)00151-1.

Maia, M. R. G. et al. (2007) 'Metabolism of polyunsaturated fatty acids and their toxicity to the microflora of the rumen', Antonie van Leeuwenhoek, International Journal of General and Molecular Microbiology, 91(4), pp. 303-314. doi: 10.1007/s10482-006-9118-2.

Makkonen, M., Berg, M. P., Van Hal, J. R., Callaghan, T. V., Press, M. C., \& Aerts, R. (2011) 'Traits explain the responses of a sub', Soil Biology and Biochemistry, 43(2), pp. 377-384.

Maraun, M. et al. (2011) 'Stable isotopes revisited: Their use and limits for oribatid mite trophic ecology', Soil Biology and Biochemistry, 43(5), pp. 877-882. doi: 10.1016/j.soilbio.2011.01.003.

Margono, B. A. et al. (2014) 'Primary forest cover loss in indonesia over 2000-2012', Nature Climate Change, 4(8), pp. 730-735. doi: 10.1038/nclimate2277.

Marian, F. et al. (2017) 'Leaf and root litter decomposition is discontinued at high altitude tropical montane rainforests contributing to carbon sequestration', (January), pp. 6432-6443. doi: 10.1002/ece3.3189.

Marian, F. et al. (2018) 'Altitude and decomposition stage rather than litter origin structure soil microarthropod communities in tropical montane rainforests', Soil Biology and Biochemistry. Elsevier, 125(May), pp. 263-274. doi: 10.1016/j.soilbio.2018.07.017.

Marimin et al. (2014) 'Value chain analysis for green productivity improvement in the natural rubber supply chain: A case study', Journal of Cleaner Production. Elsevier Ltd, 85, pp. 201-211. doi: 10.1016/j.jclepro.2014.01.098.

Martius, C. et al. (2004) 'Microclimate in agroforestry systems in central Amazonia : 
does canopy closure matter to soil organisms ?', Agroforestry Systems, pp. 291-304.

Mateos, E. and Greenslade, P. (2015) 'Towards understanding Lepidocyrtus Bourlet, 1839 (Collembola, Entomobryidae) I: Diagnosis of the subgenus Setogaster, new records and redescriptions of species', Zootaxa, 4044(1), pp. 105-129. doi: 10.11646/zootaxa.4044.1.6.

Matson, P. A. et al. (1997) 'Agricultural intensification and ecosystem properties', Science, 277(5325), pp. 504-509. doi: 10.1126/science.277.5325.504.

Mayvan, M. M., Shayanmehr, M. and Scheu, S. (2015) 'Depth distribution and interannual fluctuations in density and diversity of Collembola in an Iranian Hyrcanian forest', 87(December), pp. 239-247.

Meijide, A., Badu, C. S., Moyano, F., Tiralla, N., Gunawan, D., \& Knohl, A. (2018) 'Impact of forest conversion to oil palm and rubber plantations on microclimate and the role of the 2015 ENSO event', Agricultural and forest meteorology, 252, pp. 208-219. Available at: https://doi.org/10.1016/j.agrformet.2018.01.013.

Menzel, R., Ngosong, C. and Ruess, L. (2017) 'Isotopologue profiling enables insights into dietary routing and metabolism of trophic biomarker fatty acids', Chemoecology. Springer International Publishing, 27(3), pp. 101-114. doi: 10.1007/s00049-017-02362.

Michon, G. and de Foresta, H. (1995) Conserving Biodiversity Outside Protected Areas : The Indonesian agro-forest model, Encyclopedia of Biodiversity: Second Edition. doi: 10.1016/B978-0-12-384719-5.00359-2.

Miettinen, J., Shi, C. and Liew, S. C. (2011) 'Deforestation rates in insular Southeast Asia between 2000 and 2010', Global Change Biology, 17(7), pp. 2261-2270. doi: 10.1111/j.1365-2486.2011.02398.x.

Millar, A. A., Smith, M. A. and Kunst, L. (2000) 'All fatty acids are not equal: Discrimination in plant membrane lipids', Trends in Plant Science, 5(3), pp. 95-101. doi: 10.1016/S1360-1385(00)01566-1.

Monroy, F., Aira, M. and Domínguez, J. (2008) 'Changes in density of nematodes, protozoa and total coliforms after transit through the gut of four epigeic earthworms (Oligochaeta)', Applied Soil Ecology, 39(2), pp. 127-132. doi:

10.1016/j.apsoil.2007.11.011.

Moore, J. C. et al. (2004) 'Detritus, trophic dynamics and biodiversity', Ecology Letters, 7(7), pp. 584-600. doi: 10.1111/j.1461-0248.2004.00606.x.

Moore, J. C. and De Ruiter, P. C. (2012) Energetic food webs:an analysis of real and model ecosystems.

Muturi, J. J., Mbugi, J. P., Mueke, J. M., Lagerlóf, J., Mungatu, J. K., Nyamasyo, G., \& Gikungu, M. (2009) 'Collembola density and diversity along a gradient of land-use types in Embu district, Eastern Kenya', Tropical and Subtropical Agroecosystems, 11(2), pp. 361-369.

Muturi, J. J. et al. (2011) 'Effect of integrated soil fertility management interventions on the abundance and diversity of soil Collembola in Embu and Taita Districts, Kenya', Tropical and Subtropical Agroecosystems, 13(1), pp. 35-42. 
Myers, N. et al. (2000) 'Biodiversity hotspots for conservation priorities', Nature, 403(6772), pp. 853-858. doi: 10.1038/35002501.

Nacke, H. et al. (2011) 'Pyrosequencing-based assessment of bacterial community structure along different management types in German forest and grassland soils', PLoS ONE, 6(2). doi: 10.1371/journal.pone.0017000.

Nathalie, C., Charles, G., and Thierry, G. (2003) 'Relationships between Collembola, soil chemistry and humus types in forest stands ( France )', pp. 355-361. doi: 10.1007/s00374-003-0610-9.

Ngosong, C. et al. (2009) 'Low importance for a fungal based food web in arable soils under mineral and organic fertilization indicated by Collembola grazers', Soil Biology and Biochemistry, 41(11), pp. 2308-2317. doi: 10.1016/j.soilbio.2009.08.015.

Oelbermann, K., Langel, R. and Scheu, S. (2008) 'Utilization of prey from the decomposer system by generalist predators of grassland', Oecologia, 155(3), pp. 605617. doi: 10.1007/s00442-007-0927-4.

Olsson, P. A. (1999) 'Signature fatty acids provide tools for determination of the distribution and interaction of mycorrhizal fungi', FEMS Microbiology Ecology, 29(August), pp. 303-310. doi: 10.1111/j.1574-6941.1999.tb00621.x.

Or-Rashid, M. M., AlZahal, O. and McBride, B. W. (2008) 'Studies on the production of conjugated linoleic acid from linoleic and vaccenic acids by mixed rumen protozoa', Applied Microbiology and Biotechnology, 81(3), pp. 533-541. doi: 10.1007/s00253008-1690-0.

Or-Rashid, M. M., Odongo, N. E. and McBride, B. W. (2007) 'Fatty acid composition of ruminai bacteria and protozoa, with emphasis on conjugated linoleic acid, vaccenic acid, and odd-chain and branched-chain fatty acids', Journal of Animal Science, 85(5), pp. 1228-1234. doi: 10.2527/jas.2006-385.

Palacios-Vargas, J. G. and Castaño-Meneses, G. (2003) 'Seasonality and community composition of springtails in Mexican forests', Arthropods of tropical forests: spatiotemporal dynamics and resource use in the canopy, pp. 159-169.

Panizzi, A. R., Beatriz, S. and Ferreira, C. (1997) 'Dynamics in the insect fauna adaptation to sybean in the tropics'.

Persson, T. (1989) 'Role of soil animals in C and N mineralisation', Plant and Soil, 115(2), pp. 241-245. doi: 10.1007/BF02202592.

Petersen, H. (2011) 'Collembolan communities in shrublands along climatic gradients in Europe and the effect of experimental warming and drought on population density, biomass and diversity', Soil Organisms, 83(3), pp. 463-488.

Petersen, S. O. and Holmstrup, M. (2000) 'Temperature effects on lipid composition of the earthworms Lumbricus rubellus and Eisenia nordenskioeldi', Soil Biology and Biochemistry, 32(11-12), pp. 1787-1791. doi: 10.1016/S0038-0717(00)00059-6.

Pey, B. et al. (2014) 'Current use of and future needs for soil invertebrate functional traits in community ecology', Basic and Applied Ecology, 15(3), pp. 194-206. doi: 10.1016/j.baae.2014.03.007.

Pokarzhevskii, A. D. (1997) 'Aminoacidos in earthworms : Are earthworms 
ecosistemivorous?', Soil Biology and Biochemistry, 29(3/4), pp. 559-567. doi: 10.1016/S0038-0717(96)00180-0.

Pollierer, M. M. et al. (2009) 'Compartmentalization of the soil animal food web as indicated by dual analysis of stable isotope ratios (15N/14N and 13C/12C)', Soil Biology and Biochemistry, 41(6), pp. 1221-1226. doi: 10.1016/j.soilbio.2009.03.002.

Pollierer, M. M. et al. (2012) 'Carbon flux through fungi and bacteria into the forest soil animal food web as indicated by compound-specific 13C fatty acid analysis', Functional Ecology, 26(4), pp. 978-990. doi: 10.1111/j.1365-2435.2012.02005.x.

Pollierer, M. M., Ferlian, O. and Scheu, S. (2015) 'Temporal dynamics and variation with forest type of phospholipid fatty acids in litter and soil of temperate forests across regions', Soil Biology and Biochemistry, pp. 248-257. doi:

10.1016/j.soilbio.2015.08.035.

Pollierer, M. M. and Scheu, S. (2017) 'Driving factors and temporal fluctuation of Collembola communities and reproductive mode across forest types and regions', Ecology and Evolution, 7(12), pp. 4390-4403. doi: 10.1002/ece3.3035.

Pollierer, M. M., Scheu, S. and Haubert, D. (2010) 'Taking it to the next level: Trophic transfer of marker fatty acids from basal resource to predators', Soil Biology and Biochemistry. Elsevier Ltd, 42(6), pp. 919-925. doi: 10.1016/j.soilbio.2010.02.008.

Ponge, J.-F. (1983) 'Les collemboles, indicateurs du type d'humus en milieu forestier: résultats obtenus au Sud de Paris (in French, with English summary)', Acta Oecologica, Oecologia Generalis, 4(4), pp. 359-374. Available at: https://www.researchgate.net/publication/264877236.

Ponge, J. F. (2000a) 'The use of phospholipid fatty acid analysis to estimate bacterial and fungal biomass in soil', Biol Fertil Soils, 32, pp. 508-522.

Ponge, J. F. (2000b) 'Vertical distribution of collembola (Hexapoda) and their food resources in organic horizons of beech forests', Biology and Fertility of Soils, 32(6), pp. 508-522. doi: 10.1007/s003740000285.

Ponge, J. F., Arpin, P. and Vannier, G. (1993) 'Collembolan response to experimental perturbations of litter supply in a temperate forest ecosystem', European Journal of Soil Biology, 29(3-4), pp. 141-153.

Ponge, J. F. and Salmon, S. (2013) 'Spatial and taxonomic correlates of species and species trait assemblages in soil invertebrate communities', Pedobiologia, 56(3), pp. 129-136. doi: 10.1016/j.pedobi.2013.02.001.

Potapov, A. et al. (2020) 'Towards a global synthesis of Collembola knowledge: challenges and potential solutions', Soil Organisms, 92(3), pp. 161-188. doi: 10.25674/so92iss3pp161.

Potapov, A. M. et al. (2013a) 'Large 13C/12C and small 15N/14N isotope fractionation in an experimental detrital foodweb (litter-fungi-collembolans)', Ecological Research, 28(6), pp. 1069-1079. doi: 10.1007/s11284-013-1088-z.

Potapov, A. M. et al. (2013b) 'Large13C/12C and small15N/14N isotope fractionation in an experimental detrital foodweb (litter-fungi-collembolans)', Ecological Research, 28(6), pp. 1069-1079. doi: 10.1007/s11284-013-1088-z. 
Potapov, A. A. et al. (2016a) 'Connecting taxonomy and ecology: Trophic niches of collembolans as related to taxonomic identity and life forms', Soil Biology and Biochemistry. Elsevier Ltd, 101, pp. 20-31. doi: 10.1016/j.soilbio.2016.07.002.

Potapov, A. M. et al. (2016b) 'Assimilation of plant-derived freshly fixed carbon by soil collembolans: Not only via roots?', Pedobiologia. Elsevier GmbH., 59(4), pp. 189-193. doi: 10.1016/j.pedobi.2016.07.002.

Potapov, A. M., Dupérré, N., et al. (2019) 'Functional losses in ground spider communities due to habitat-structure degradation under tropical land-use change', Ecology. doi: 10.1002/ecy.2957.

Potapov, A. M., Klarner, B., et al. (2019) 'Linking size spectrum, energy flux and trophic multifunctionality in soil food webs of tropical land-use systems', Journal of Animal Ecology, 88(12), pp. 1845-1859. doi: 10.1111/1365-2656.13027.

Potapov, A. M. et al. (2020a) 'Functional losses in ground spider communities due to habitat structure degradation under tropical land-use change', Ecology, 101(3), pp. 114. doi: 10.1002/ecy.2957.

Potapov, A. M. et al. (2020b) 'Ground Spider Communities Under Tropical Land-Use Change', The Bulletin of the Ecological Society of America, 101(2), pp. 1-3. doi: 10.1002/bes2.1668.

Potapov, A. M., Korotkevich, A. Y. and Tiunov, A. V. (2018a) 'Non-vascular plants as a food source for litter-dwelling Collembola: Field evidence', Pedobiologia. Elsevier, 66(August 2017), pp. 11-17. doi: 10.1016/j.pedobi.2017.12.005.

Potapov, A. M. and Tiunov, A. V. (2016) 'Stable isotope composition of mycophagous collembolans versus mycotrophic plants: do soil invertebrates feed on mycorrhizal fungi?.', Soil Biology and Biochemistry, 93, pp. 115-118.

Potapov, A. M., Tiunov, A. V. and Scheu, S. (2018b) 'Uncovering trophic positions and food resources of soil animals using bulk natural stable isotope composition', Biological Reviews. doi: 10.1111/brv.12434.

Potapov, M. (2012) 'Taxonomy of the', Transactions of the American Microscopical Society, 49(1), pp. 38-49.

Potapov, M. B. and Starostenko, E. V. (2002) 'Taxonomical notes on the species of the genus Isotomurus (Collembola: Isotomidae) with the "balteatus"-like colouration', Russian Entomological Journal, 11(4), pp. 331-333.

Rawlins, A. J. et al. (2006) 'The biochemical transformation of oak (Quercus robur) leaf litter consumed by the pill millipede (Glomeris marginata)', Soil Biology and Biochemistry, 38(5), pp. 1063-1076. doi: 10.1016/j.soilbio.2005.09.005.

Raymond-Léonard, L. J., Gravel, D. and Handa, I. T. (2019) 'A novel set of traits to describe Collembola mouthparts: taking a bite out of the broad chewing mandible classification', Soil Biology and Biochemistry, 138(September). doi: 10.1016/j.soilbio.2019.107608.

Reddy, M. V. et al. (1994) 'Decomposition of straw in relation to tillage, moisture, and arthropod abundance in a semi-arid tropical Alfisol', Biology and Fertility of Soils, 17(1), pp. 45-50. doi: 10.1007/BF00418671. 
Rembold, K. et al. (2017) 'Plant diversity, forest dependency, and alien plant invasions in tropical agricultural landscapes', Biological Conservation. Elsevier, 213(July), pp. 234-242. doi: 10.1016/j.biocon.2017.07.020.

Rodrigues, J. L. M. et al. (2013) 'Conversion of the Amazon rainforest to agriculture results in biotic homogenization of soil bacterial communities', Proceedings of the National Academy of Sciences of the United States of America, 110(3), pp. 988-993. doi: 10.1073/pnas.1220608110.

Rojas, A. B. et al. (2009) 'Oribatid mites and springtails from a coffee plantation in Sierra Sur, Oaxaca, Mexico', Pesquisa Agropecuária Brasileira, 44(8), pp. 988-995. doi: 10.1590/s0100-204x2009000800030.

Rousk, J. et al. (2010) 'Soil bacterial and fungal communities across a pH gradient in an arable soil', ISME Journal, 4(10), pp. 1340-1351. doi: 10.1038/ismej.2010.58.

Rousk, J. and Bååth, E. (2007) 'Fungal and bacterial growth in soil with plant materials of different C/N ratios', FEMS Microbiology Ecology, 62(3), pp. 258-267. doi: 10.1111/j.1574-6941.2007.00398.x.

Ruess, L. et al. (2002) 'acids of fungi and nematodes_-possible biomarkers in the soil food chain?', Soil Biology and Biochemistry, 34(6), pp. 745-756. doi: 10.1016/S00380717(01)00231-0.

Ruess, L. et al. (2004) 'Nitrogen isotope ratios and fatty acid composition as indicators of animal diets in belowground systems', Oecologia, 139(3), pp. 336-346. doi: 10.1007/s00442-004-1514-6.

Ruess, L et al. (2005) 'Application of lipid analysis to understand trophic interactions in soil’, Ecology, 86(8), pp. 2075-2082. doi: 10.1890/04-1399.

Ruess, Liliane et al. (2005) 'Carbon stable isotope fractionation and trophic transfer of fatty acids in fungal based soil food chains', Soil Biology and Biochemistry, 37(5), pp. 945-953. doi: 10.1016/j.soilbio.2004.09.015.

Ruess, L. et al. (2007) 'Lipid composition of Collembola and their food resources in deciduous forest stands-Implications for feeding strategies', Soil Biology and Biochemistry, 39(8), pp. 1990-2000. doi: 10.1016/j.soilbio.2007.03.002.

Ruess, L. and Chamberlain, P. M. (2010) 'The fat that matters: Soil food web analysis using fatty acids and their carbon stable isotope signature', Soil Biology and Biochemistry. Elsevier Ltd, 42(11), pp. 1898-1910. doi: 10.1016/j.soilbio.2010.07.020.

De Ruiter, P. C. et al. (1993) 'Calculation of nitrogen mineralization in soil food webs', Plant and Soil, pp. 263-273. doi: 10.1007/bf00011055.

Ruiter, P. C. De, Neutel, A. and Moore, J. C. (1995) 'Energetics, Patterns of Interaction Strengths, and Stability in Real Ecosystems', Advancement Of Science, 269(5228), pp. $1257-1260$.

Rusek, J. (1998) 'Biodiversity of Collembola and their functional role in the ecosystem', Biodiversity and Conservation, 7(9), pp. 1207-1219. doi: 10.1023/A:1008887817883.

Rusek, J. (2007) 'A new classification of Collembola and Protura life forms. In: contribution to soil zoology in central Europe II.', ISB BC ASCR. Ceske Budejovice, pp. 
$109-115$.

Sahner, J. et al. (2015) 'Degradation of root community traits as indicator for transformation of tropical lowland rain forests into oil palm and rubber plantations', PLoS ONE, 10(9), pp. 1-19. doi: 10.1371/journal.pone.0138077.

Salamon, J. A., Scheu, S., and Schaefer, M. (2008) 'The Collembola community of pure and mixed stands of beech'. Pedobiologia, pp. 51(5-6), 385-396.

Salmon, S. et al. (2014) 'Linking species, traits and habitat characteristics of Collembola at European scale', Soil Biology and Biochemistry. Elsevier Ltd, 75, pp. 7385. doi: 10.1016/j.soilbio.2014.04.002.

Salmon, S. and Ponge, J. F. (2012) 'Species traits and habitats in springtail communities: A regional scale study’, Pedobiologia. Elsevier GmbH., 55(6), pp. 295 301. doi: 10.1016/j.pedobi.2012.05.003.

Sampedro, L., Jeannotte, R. and Whalen, J. K. (2006) 'Trophic transfer of fatty acids from gut microbiota to the earthworm Lumbricus terrestris L', Soil Biology and Biochemistry, 38(8), pp. 2188-2198. doi: 10.1016/j.soilbio.2006.02.001.

Scheu, S. and Falca, M. (2000) 'The soil food web of two beech forests ( Fagus sylvatica ) of contrasting humus type: stable isotope analysis of a macro- and a mesofauna-dominated community', Oecologia, 123(2), pp. 285-296. doi: $10.1007 / \mathrm{s} 004420051015$.

Scheu, S. and Folger, M. (2004) 'Single and mixed diets in Collembola : Effects on reproduction and stable isotope fractionation Single and mixed diets in Collembola : effects on reproduction and stable isotope fractionation', (October 2016), pp. 94-102. doi: 10.1046/j.0269-8463.2004.00807.x.

Schmidt, O., Dyckmans, J. and Schrader, S. (2016) 'Photoautotrophic microorganisms as a carbon source for temperate soil invertebrates Photoautotrophic microorganisms as a carbon source for temperate soil invertebrates', Bology Letters, 12(JANUARY), p. 20150646. doi: 10.1098/rsbl.2015.0646.

Schneider, D. et al. (2015) 'Impact of lowland rainforest transformation on diversity and composition of soil prokaryotic communities in sumatra (Indonesia)', Frontiers in Microbiology, 6(DEC), pp. 1-12. doi: 10.3389/fmicb.2015.01339.

Selvany, R. (2018) Kelimpahan dan keanekaragaman collembola pada lima tipe penggunaan lahan di kapuas hulu kalimantan barat remila selvany.

Semenyuk, I., Tiunov, A. V. and Golovatch, S. (2011) 'Structure of mandibles in relation to trophic niche differentiation in a tropical millipede community', International Journal of Myriapodology, 6, pp. 37-49. doi: 10.3897/ijm.6.2214.

Shen, J. P. et al. (2012) 'A review of ammonia-oxidizing bacteria and archaea in Chinese soils', Frontiers in Microbiology, 3(AUG), pp. 1-7. doi: 10.3389/fmicb.2012.00296.

Sigsgaard, L. (2000) 'Early season natural biological control of insect pests in rice by spiders - and some factors in the management of the cropping system that may affect this control', European Arachnology, 2000(July 2000), pp. 57-64.

Smit, C. E. and Van Gestel, C. A. M. (1997) 'Influence of temperature on the regulation 
and toxicity of zinc in (Folsomia candida (Collembola)', Ecotoxicology and Environmental Safety, 37(3), pp. 213-222. doi: 10.1006/eesa.1997.1558.

Sodhi, N. S. et al. (2004) 'Southeast Asian biodiversity: An impending disaster', Trends in Ecology and Evolution, 19(12), pp. 654-660. doi: 10.1016/j.tree.2004.09.006.

Son, J., Shin, K. il and Cho, K. (2009) 'Response surface model for predicting chronic toxicity of cadmium to Paronychiurus kimi (Collembola), with a special emphasis on the importance of soil characteristics in the reproduction test', Chemosphere. Elsevier Ltd, 77(7), pp. 889-894. doi: 10.1016/j.chemosphere.2009.08.047.

Sousa, J. P. et al. (2006) 'Changes in Collembola richness and diversity along a gradient of land-use intensity: A pan European study', Pedobiologia, 50(2), pp. 147-156. doi: 10.1016/j.pedobi.2005.10.005.

Van Straalen NM, Timmermans MJTN, Roelofs D, B. M. (2008) 'Apterygota in the spotlights of ecology, evolution and genomics', Europian Journal Soil Biology, 44, pp. $452-457$.

van Straaten, O. et al. (2015) 'Conversion of lowland tropical forests to tree cash crop plantations loses up to one-half of stored soil organic carbon', Proceedings of the National Academy of Sciences, 112(32), pp. 9956-9960. doi: 10.1073/pnas.1504628112.

Suhardjono, Y. R., Deharveng, L. and Bedos, A. (2012) 'Collembola (ekor pegas): Biologi, klasifikasi, ekologi'.

Susanti, W. I. et al. (2019) 'Conversion of rainforest to oil palm and rubber plantations alters energy channels in soil food webs', Ecology and Evolution. doi: 10.1002/ece3.5449.

Susanti, W. I. et al. (2021) 'Trophic niche differentiation and utilisation of food resources in Collembola is altered by rainforest conversion to plantation systems', PeerJ, 9, pp. 1-18. doi: 10.7717/peerj.10971.

Susanti, W. I., Bartels, T., Krashevska, V., Widyastuti, R., Deharveng, L., Scheu, S., Potapov, A. (2021) 'Conversion of rainforest to rubber and oil palm plantations affects the functional composition of litter and soil Collembola', Ecology and Evolution. doi.org/10.1002/ece3.7881.

Tao, H. H., Snaddon, J. L., Slade, E. M., Henneron, L., Caliman, J. P., \& Willis, K. J. (2018) 'Application of oil palm empty fruit bunch effects on soil biota and functions: A case study in Sumatra, Indonesia', Agriculture, ecosystem \& environment, 256, pp. 105113.

Team, R. C. (2017) R: A language and environment for statistical computing. $R$ Foundation for Statistical Computing. Vienna, Austria. Available at: https://www.rproject.org/.

Titeux, N. et al. (2016) 'Biodiversity scenarios neglect future land-use changes', Global change biology, 22(7), pp. 2505-2515. doi: 10.1111/gcb.13272.

Tiunov, A. V. (2007) 'Stable isotopes of carbon and nitrogen in soil ecological studies', Biology Bulletin, 34(4), pp. 395-407. doi: 10.1134/S1062359007040127.

Tsurikov, S. M., Ermilov, S. G. and Tiunov, A. V. (2019) 'Trophic structure of a 
tropical soil- and litter-dwelling oribatid mite community and consistency of trophic niches across biomes', Experimental and Applied Acarology. Springer International Publishing, 78(1), pp. 29-48. doi: 10.1007/s10493-019-00374-4.

Tylianakis, J. M., Tscharntke, T. and Lewis, O. T. (2007) 'Habitat modification alters the structure of tropical host-parasitoid food webs', Nature, 445(7124), pp. 202-205. doi: 10.1038/nature05429.

Vandewalle, M. et al. (2010) 'Functional traits as indicators of biodiversity response to land use changes across ecosystems and organisms', Biodiversity and Conservation, 19(10), pp. 2921-2947. doi: 10.1007/s10531-010-9798-9.

Violita, V. et al. (2016) 'Fine Root Production and Decomposition in Lowland Rainforest and Oil Palm Plantations in Sumatra , Indonesia', HAYATI Journal of Biosciences. Elsevier Ltd, 23(1), pp. 7-12. doi: 10.1016/j.hjb.2015.10.008.

Violle, C. et al. (2007) 'Let the concept of trait be functional!', Oikos, (January), pp. 111. doi: 10.1111/j.2007.0030-1299.15559.x.

Visser, S., Whittaker, J. B. and Parkinson, D. (1981) 'Effects of collembolan grazing on nutrient release and respiration of a leaf litter inhabiting fungus', Soil Biology and Biochemistry, 13(3), pp. 215-218. doi: 10.1016/0038-0717(81)90023-7.

Voigtländer, K. (2011) 'Chilopoda: ecology', Treatise on Zoology--Anatomy, Taxonomy, Biology: The Myriapoda, 1, pp. 309-325.

De Vries, F. T. et al. (2013) 'Soil food web properties explain ecosystem services across European land use systems', Proceedings of the National Academy of Sciences of the United States of America, 110(35), pp. 14296-14301. doi:

10.1073/pnas.1305198110.

Wagg, C. et al. (2014) 'Soil biodiversity and soil community composition determine ecosystem multifunctionality.', Proceedings of the National Academy of Sciences of the United States of America, 111(14), pp. 5266-70. doi: 10.1073/pnas.1320054111.

Wakeham, S. G., Pease, T. K. and Benner, R. (2003) 'Hydroxy fatty acids in marine dissolved organic matter as indicators of bacterial membrane material', Organic Geochemistry, 34, pp. 857-868.

Wang, G. M. et al. (1993) 'Effects of pH on arbuscular mycorrhiza I. Field observations on the long-term liming experiments at Rothamsted and Woburn', New Phytologist, 124(3), pp. 465-472. doi: 10.1111/j.1469-8137.1993.tb03837.x.

Wardle, D. A. et al. (2004) 'Belowground BiotaEcological Linkages Between Aboveground and Belowground Biota', Science (New York, N.Y.), 304(June), pp. 1629-1633. doi: 10.1126/science.1094875.

Warino, J. et al. (2017) 'Keanekaragaman dan kelimpahan Collembola pada perkebunan kelapa sawit di Kecamatan Bajubang, Jambi', Jurnal Entomologi Indonesia, 14(2), pp. 51-57. doi: 10.5994/jei.14.2.51.

Welch, D. F. (1991) 'Applications of cellular fatty acid analysis', Clin.Microbiol.Rev., 4(4), pp. 422-438.

Widrializa et al. (2015) 'The Diversity and Abundance of Springtail (Collembola) on Forests and Smallholder in Jambi', Jurnal Tanah Tropika, 20(3), pp. 173-180. doi: 
10.5400/jts.2015.20.3.

Wilcove, D. S. and Koh, L. P. (2010) 'Addressing the threats to biodiversity from oilpalm agriculture', Biodiversity and Conservation, 19(4), pp. 999-1007. doi:

10.1007/s10531-009-9760-x.

Winck, B. R., de Sá, E. L. S., Rigotti, V. M., \& Chauvat, M. (2017) 'Relationship between land-use types and functional diversity of epigeic Collembola in Southern Brazil', Applied Soil Ecology, 109, pp. 49-59. Available at:

https://doi.org/10.1016/j.apsoil.2016.09.021.

Wise, D. . (2004) 'Wandering spiders limit densities of a major microbi-detritivore in the forest-floor food web', Pedobiologia, 48(2), pp. 181-188.

Wise, D. H. (2006) 'Using Stable Isotopes To Reveal Shifts In Prey Consumption By Generalist Predators', Ecological Applications, 27(3), pp. 363-876. doi: 10.1890/10510761(2006)016[0865:USITRS]2.0.CO;2.

Wiwatwitaya, D. and Takeda, H. (2005) 'Seasonal changes in soil arthropod abundance in the dry evergreen forest of north-east Thailand, with special reference to collembolan communities', Ecological Research, 20(1), pp. 59-70. doi: 10.1007/s11284-004-0013$\mathrm{x}$.

Xu, G. L. et al. (2012) 'Seasonal exposure to drought and air warming affects soil collembola and mites', PLOS ONE, 7(8), pp. 23-27. doi: 10.1371/journal.pone.0043102.

Zelles, L. (1999) 'Fatty acid patterns of phospholipids and lipopolysaccharides in the characterization of microbial communities in soil: A review', Biology and Fertility of Soils, (29), pp. 111-129. doi: 10.1007/s003740050533.

Zhang, X. F. et al. (2013) 'Soil moisture effect on bacterial and fungal community in Beilu River (Tibetan Plateau) permafrost soils with different vegetation types', Journal of Applied Microbiology, 114(4), pp. 1054-1065. doi: 10.1111/jam.12106.

Zieger, S. L. and Scheu, S. (2018) 'Effects of storage and handling on neutral lipid fatty acid profiles of two woodlice (Isopoda, Crustacea) species differing in size', Applied Soil Ecology, 130(December 2017), pp. 178-184. doi: 10.1016/j.apsoil.2018.04.009. 


\section{DANKSAGUNG}

Ich möchte Stefan Scheu dafür danken, ein hervorragender Professor und recht guter Mensch zu sein. Er gab mir die Möglichkeit, in Göttingen zu promovieren. Ich danke ihm ebenso für viele inspirierende und motivierende Diskussionen und er fand stets Lösungen für alle möglichen Probleme und Hürde im Labor, im Feld und im alltäglichen Leben in Deutschland. Ein schönes Dankeschön sende ich auch an Christoph Bleidorn als mein guter Betreuer für seine wertvollen Anregungen und Zeit während der Promotion.

Herzlichen Dank an Mark Maraun, Holger Kreft, Sven Bradler, und Klaus Hövemeyer als Prüfungskommission für die wertvolle Zeit in meiner Disputation.

Es ist Anton Potapov zu verdanken für seine Hilfsbereitschaft. Ich hatte eine großartige Zeit mit ihm und anderen Studenten im Identifikationskurs. Da habe ich zum ersten Mal Collembola kennengelernt. Er hat mich in das EFForTS-Projekt eingeführt und war in all den Jahren meiner Promotion sehr hilfreich. Ebenso würde ich ihm für das Geduld beim Unterricht der Statistik und des wissenschaftlichen Schreibens danken.

Ich möchte mich auch bei Melanie Mira Pollierer für die wertvolle Zeit bedanken, in der sie mir am Anfang beim Fettsäure-Experiment helfen. Das werde ich nicht vergessen. Ich werde mich immer an ihr Lächeln erinnern, als ich das Labor zum ersten Mal betrat. Ich möchte auch meinem Lieblings-TA, Guido Humpert, für die Hilfe danken. Ich werde mich immer daran erinnern, als er mir beigebracht hat, mit den FAs umzugehen.

Das nächste Dankeschön geht an Dorothee Sandmann, ich weiß nicht, wie ich ohne sie mein Leben als Studentin mit Baby in Göttingen weiterkämpfen sollte. Sie hat mir all ihre Erfahrungen damit mitgeteilt.

Danke auch an Franca, Garvin und Valentyna, die mir vorgeschlagen haben. Die gemeinsamen Diskussionen mit ihnen gefielen mir immer. Es machte außerdem wirklich Spaß, mit ihnen Statistik und Kochen zu lernen.

Auch Susanne, Ina Schäfer, Leonie Schärdt und Tamara Hartke möchte ich ganz herzlich für die Mithilfe bei den weiteren Laborarbeiten danken. Mein Dank gilt auch Andrea Lambertz, Dora Volovei, Barbara Wick, Ivonne Hein und Elisabeth Opielka. Ohne ihren Beitrag und ihre Organisation hätte vieles in der Arbeitsgruppe nicht reibungslos ablaufen können.

Ein besonderer Dank gilt auch Amanda Mawan, Laura Sanchez, Zhijing Xie, Jingjong $\mathrm{Lu}$, Zhipeng Li, Shixing Zhou, Linlin, Daniel Ramous Gutierres, Alena Krause, Zheng Zhou, Svenja Meyer, Melissa Jüds, Johannes Lux und Jörg Hanisch für alle Freundschaften und Hilfestellungen während meines Promotionsstudiums. Ich danke ihnen, dass sie mich immer freuen und lächeln lassen, besonders als ich schwanger war.

Bedanken möchte ich mich noch bei allen AG-Scheu-Mitgliedern, die mit mir jeden Moment während meines Doktoratslebens in Göttingen genossen haben.

$\mathrm{Zu}$ guter Letzt danke ich meinem lieben Ehemann Rizal Taufiq Fauzi und meinem Sohn M. Ibrahim Musa Alhafiz. Sie haben mich während der Promotion immer unterstützt. Vor allem an Ibrahim. Er war immer bei mir im Labor während meines Dissertation. Sie sind mein Support-System. Vielen Dank auch an alle Indonesischen Freunde in Goettingen für die Hilfe and Freundschaft während das Studiums. Anschließend danke ich auch meiner Mutter, meiner Familie und meinen indonesischen Freunden, die mich während der Promotion unterstützt haben. 


\section{List of Publication}

Susanti, W. I., Pollierer, M.M., Widyastuti, R., Scheu, S., Potapov, A. (2019) 'Conversion of rainforest to oil palm and rubber plantations alters energy channels in soil food webs', Ecology and Evolution. doi: 10.1002/ece3.5449.

Susanti, W. I., Widyastuti, R., Scheu, S., Potapov, A. (2021) 'Trophic niche differentiation and utilisation of food resources in Collembola is altered by rainforest conversion to plantation systems', PeerJ, 9, pp. 1-18. doi: 10.7717/peerj.10971.

Susanti, W. I., Bartels, T., Krashevska, V., Widyastuti, R., Deharveng, L., Scheu, S., Potapov, A. (2021) 'Conversion of rainforest to rubber and oil palm plantations affects the functional composition of litter and soil Collembola', Ecology and Evolution. doi.org/10.1002/ece3.7881.

\section{Thesis declarations}

Declaration of the author's own contribution to manuscripts with multiple authors.

Chapters 2,3 and 4 comprise a manuscript that has been published in a peer-reviewed journal. In all manuscripts that presented in Chapter 2, 3 and 4, I am the first author and I have analyzed the data, written the manuscripts, developed the main ideas, created tables, figures and appendices and contributed significantly to the study design. All coauthors contributed to finalizing the manuscripts have been named so.

The study design was developed in the framework of the Collaborative Research Centre 990: Ecological and Socioeconomic Functions of Tropical Lowland Rainforest Transformation Systems (Sumatra, Indonesia). EFForTS is a Collaborative Research Centre 990 funded by the Deutsche Forschungsgemeinschaft (DFG, German Research Foundation) that investigates the ecological and socio-economic effects of such rainforest transformation in Indonesia.

\section{Plagiarism declaration}

I declare that I have written this doctoral thesis independently. All persons contributing to the manuscripts have been named so. All sentences or passages quoted from other people's work have been specifically acknowledged by clear cross-referencing. I have not submitted this thesis in any form for another degree at any university or institution.

Winda Ika Susanti

Göttingen, September 2021 
